CCNY-HEP-94/03

KUL-TF-94/12

UB-ECM-PF 94/15

UTTG-11-94

hep-th/9412228

May 1994

\title{
Antibracket, Antifields and Gauge-Theory Quantization
}

\author{
Joaquim Gomis ${ }^{* 1}$, Jordi París ${ }^{\sharp 2}$ and Stuart Samuel ${ }^{\dagger 3}$ \\ * Theory Group, Department of Physics \\ The University of Texas at Austin \\ RLM 5208, Austin, Texas \\ and \\ Departament d'Estructura i Constituents de la Matèria \\ Facultat de Física, Universitat de Barcelona \\ Diagonal 647, E-08028 Barcelona \\ Catalonia \\ \# Instituut voor Theoretische Fysica \\ Katholieke Universiteit Leuven \\ Celestijnenlaan 200D \\ B-3001 Leuven, Belgium \\ $\dagger$ Department of Physics \\ City College of New York \\ 138th St and Convent Avenue \\ New York, New York 10031 U.S.A.
}

\footnotetext{
${ }^{1}$ Permanent address: Dept. d'Estructura i Constituents de la Matèria, U. Barcelona. E-mail: gomis@rita.ecm.ub.es

${ }^{2}$ Wetenschappelijk Medewerker, I. I. K. W., Belgium.

E-mail: jordi=paris\%tf\%fys@cc3.kuleuven.ac.be

${ }^{3}$ E-mail: samuel@scisun.sci.ccny.cuny.edu
} 


\begin{abstract}
The antibracket formalism for gauge theories, at both the classical and quantum level, is reviewed. Gauge transformations and the associated gauge structure are analyzed in detail. The basic concepts involved in the antibracket formalism are elucidated. Gauge-fixing, quantum effects, and anomalies within the field-antifield formalism are developed. The concepts, issues and constructions are illustrated using eight gauge-theory models.
\end{abstract}




\section{Contents}

$\begin{array}{lll}1 & \text { Introduction } & 2\end{array}$

2 Structure of the Set of Gauge Transformations 10

2.1 Gauge Transformations . . . . . . . . . . . . . . . . . . . . . 11

2.2 Irreducible and Reducible Gauge Theories . . . . . . . . . . . . . . . 14

2.3 Trivial Gauge Transformations . . . . . . . . . . . . . . . . . . . . . . 17

2.4 The Gauge Structure . . . . . . . . . . . . . . . . . . . . . . . . . . . . 18

3 Examples of Gauge Theories 24

3.1 The Spinless Relativistic Particle . . . . . . . . . . . . . . . . . . . . 24

3.2 Yang-Mills Theories . . . . . . . . . . . . . . . . . . . . 27

3.3 Topological Yang-Mills Theory . . . . . . . . . . . . . . . . . . . . . . 29

3.4 The Antisymmetric Tensor Field Theory . . . . . . . . . . . . . . . . 31

3.5 Abelian $p$-Form Theories . . . . . . . . . . . . . . . . . . 33

3.6 Open Bosonic String Field Theory . . . . . . . . . . . . . . . . . . . . 34

3.7 The Massless Relativistic Spinning Particle . . . . . . . . . . . . . . . 38

3.8 The First-Quantized Bosonic String . . . . . . . . . . . . . . . . . . . 44

\begin{tabular}{|lll}
\hline & The Field-Antifield Formalism & 48
\end{tabular}

4.1 Fields and Antifields . . . . . . . . . . . . . . . . . . . . . . . . . . . 48

4.2 The Antibracket . . . . . . . . . . . . . . . . . . . . . . . . . . . . . . . 49

4.3 Classical Master Equation and Boundary Conditions . . . . . . . . . 51

4.4 The Proper Solution and the Gauge Algebra . . . . . . . . . . . . . . 54

4.5 Existence and Uniqueness . . . . . . . . . . . . . . . . . . . . . 56

4.6 The Classical BRST Symmetry . . . . . . . . . . . . . . . . . . . 57

5 Examples of Proper Solutions 59

5.1 The Spinless Relativistic Particle . . . . . . . . . . . . . . . . . . . . 59

5.2 Yang-Mills Theories . . . . . . . . . . . . . . . . . . . 61

5.3 Topological Yang-Mills Theory . . . . . . . . . . . . . . . . . . 62

5.4 The Antisymmetric Tensor Field Theory . . . . . . . . . . . . . . . . 62

5.5 Abelian $p$-Form Theories . . . . . . . . . . . . . . . . . . 62

5.6 Open String Field Theory . . . . . . . . . . . . . . . . . . . 63

5.7 The Massless Relativistic Spinning Particle . . . . . . . . . . . . . . . 66

5.8 The First-Quantized Bosonic String . . . . . . . . . . . . . . . . . . . 66 
$\begin{array}{lll}6 & \text { The Gauge-Fixing Fermion } & 68\end{array}$

6.1 Generalities . . . . . . . . . . . . . . . . . . . . . 68

6.2 Gauge-Fixing Auxiliary Fields . . . . . . . . . . . . . . . . . 71

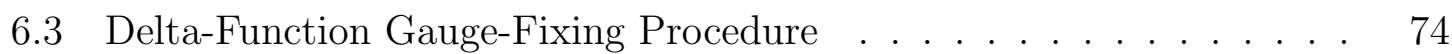

6.4 Other Gauge-Fixing Procedures . . . . . . . . . . . . . . . . . . . 83

6.5 Gauge-Fixed Classical BRST Symmetry _. . . . . . . . . . . . 85

6.6 The Gauge-Fixed Basis . . . . . . . . . . . . . . . . . . . . . 86

$\begin{array}{llr}7 & \text { Gauge-Fixing Examples } & 89\end{array}$

7.1 The Spinless Relativistic Particle $\ldots \ldots \ldots$. . . . . . . . . . 89

7.2 Yang-Mills Theories . . . . . . . . . . . . . . . . . . . . . . 90

7.3 Topological Yang-Mills Theory . . . . . . . . . . . . . . . . . . . . 91

7.4 The Antisymmetric Tensor Field Theory . . . . . . . . . . . . . 93

7.5 Open String Field Theory $\ldots \ldots \ldots \ldots$

7.6 The Massless Relativistic Spinning Particle . . . . . . . . . . . . 96

7.7 The First-Quantized Bosonic String . . . . . . . . . . . . . . . 97

8 Quantum Effects and Anomalies 101

8.1 Quantum-BRST Transformation and Its Cohomology . . . . . . . . . 101

8.2 Satisfying the Quantum Master Equation . . . . . . . . . . . . . . . 104

8.3 Remarks on Renormalization . . . . . . . . . . . . . . . . . . 105

8.4 The Effective Action and the Zinn-Justin Equation . . . . . . . . . 106

8.5 Quantum Master Equation Violations: Generalities . . . . . . . . . . 110

8.6 Canonical Transformations and the Quantum Master Equation $\ldots \ldots \ldots \ldots \ldots . \ldots \ldots 112$

8.7 The Anomaly at the One-Loop Level . . . . . . . . . . . . . . . 114

\begin{tabular}{llr}
\hline & Sample Anomaly Calculations & 119
\end{tabular}

9.1 Computation for the Spinless Relativistic Particle . . . . . . . . . . . 119

9.2 The Abelian Chiral Schwinger Model . . . . . . . . . . . . . . . . 123

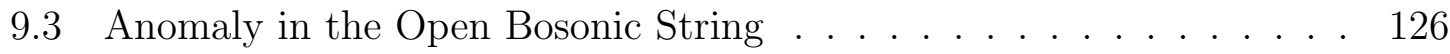

$\begin{array}{ll}10 \text { Brief Discussion of Other Topics } & 134\end{array}$

10.1 Applications to Global Symmetries . . . . . . . . . . . . . 134

10.2 A Geometric Interpretation $\ldots \ldots \ldots \ldots \ldots$

10.3 Locality . . . . . . . . . . . . . . . . . . . . . . . . . 137

10.4 Cohomological Aspects $\ldots \ldots \ldots$

10.5 Equivalence with the Hamiltonian BFV Formalism . . . . . . . . 145

10.6 Unitarity . . . . . . . . . . . . . . . . . . . . . . . . . . . 149 
10.7 The Antibracket Formalism in General Coordinates . . . . . . . . . . 153

10.8 The $\mathrm{D}=26$ Closed Bosonic String Field Theory . . . . . . . . . . . . . 155

10.9 Extended Antibracket Formalism for Anomalous Gauge Theories . . . . . . . . . . . . . . . . . . . 159

\begin{tabular}{ll}
\hline A Appendix: Right and Left Derivatives & 162
\end{tabular}

B Appendix: The Regularity Condition 164

C Appendix: Anomaly Trace Computations 166

\begin{tabular}{ll}
\hline References & 171
\end{tabular} 
This work is dedicated to Joseph and Marie, to Pilar, and to the memory of Pere and Francesca. 


\section{Introduction}

The known fundamental interactions of nature are all governed by gauge theories. The presence of a gauge symmetry indicates that a theory is formulated in a redundant way, in which certain local degrees of freedom do not enter the dynamics. Conversely, when there are degrees of freedom, which do not enter the lagrangian, a theory possesses local invariances. Although one can in principle eliminate the gauge degrees of freedom, there are reasons for not doing so. These reasons include manifest covariance, locality of interactions, and calculational convenience.

The first example of a gauge theory was electrodynamics. Electric and magnetic

forces are generated via the exchange of photons. Being particles of spin 1, photons involve a vector field, $A^{\mu}$. However, not all four components of the electromagnetic potential $A^{\mu}$ enter dynamically. Two degrees of freedom correspond to the two possible physical polarizations of the photon. The longitudinal degree of freedom plays a role in interactions via virtual exchanges of photons. The remaining gauge degree of freedom does not enter the theory. Consequently, electromagnetism is described by a gauge theory. When it was realized that the weak interactions could be unified with electromagnetism in an $S U(2) \times U(1)$ gauge theory 129, 266, 213 and that this theory is renormalizable [243, 244], the importance of non-abelian gauge theories 276] grew enormously. The strong interactions are also governed by an SU(3) non-abelian gauge theory. The fourth fundamental force is gravity. It is based on Einstein's general theory of relativity and uses general coordinate invariance. When formulated in terms of a metric or any other convenient fields, gravity also possesses gauge symmetries.

The quantization of gauge theories is not always straightforward. In the abelian case, relevant for electromagnetism, the procedure is well understood. In contrast, quantization of a non-abelian theory and its renormalization is more complicated. Quantization generally involves the introduction of ghost fields. Typically, a gaugefixing procedure is used to render dynamical all degrees of freedom. Ghost fields are used to compensate for the effects of the gauge degrees of freedom [101], so that unitarity is preserved. In electrodynamics in the linear gauges, ghosts decouple and can be ignored. In non-abelian gauge theories, convenient gauges generically involve interacting ghosts. A major step in understanding these issues was the FaddeevPopov quantization procedure [98, 83], which relied heavily on the functional-integral approach to quantization 102, 1, 165. From this viewpoint, the presence of ghost fields is understood as a "measure effect". In dividing out the volume of gauge transformations in function space, a Jacobian measure factor arises. This factor is produced naturally by introducing quadratic terms in the lagrangian for ghosts and then integrating them out. It was realized at a later stage that the gauge-fixed action 
retains a nilpotent, odd, global symmetry involving transformations of both fields and ghosts. This Becchi-Rouet-Stora-Tyutin (BRST) symmetry [36, 254] is what remains of the original gauge invariance. In fact, for closed theories, the transformation law for the original fields is like a gauge transformation with gauge parameters replaced by ghost fields. In general, this produces nonlinear transformation laws. The relations among correlation functions derived from BRST symmetry involve the insertions of the BRST variation of fields. These facts require the use of composite operators and it is convenient to introduce sources for these transformations. The Ward identities 265] associated with the BRST invariance treated in this way are the Slavnov-Taylor identities [233, 241]. The Slavnov-Taylor identities and BRST symmetry have played an important role in quantization, renormalization, unitarity, and other aspects of gauge theories.

Ghosts fields have been useful throughout the development of covariant gaugefield-theory quantization [181, 182, 184, 205, 3]. It is desirable to have a formulation of gauge theories that introduces them from the outset and that automatically incorporates BRST symmetry [32]. The field-antifield formulation has these features [36, 277, 24, 25, 26, 27]. It relies on BRST symmetry as fundamental principle and uses sources to deal with it [36, 254, 277]. It encompasses previous ideas and developments for quantizing gauge systems and extends them to more complicated situations (open algebras, reducible systems, etc.) [113, 114, 172, 238, 81]. In 1975, J. ZinnJustin, in his study of the renormalization of Yang-Mills theories [277], introduced the above-mentioned sources for BRST transformations and a symplectic structure $(,$,$) (actually denoted *$ by him) in the space of fields and sources, He expressed the Slavnov-Taylor identities in the compact form $(\Gamma, \Gamma)=0$, where $\Gamma$, the generating functional of the one-particle-irreducible diagrams, is known as the effective action (see also [187]). These ideas were developed further by B. L. Voronov and I. V. Tyutin in [263, 264 and by I. A. Batalin and G. A. Vilkovisky in refs. 24, 25, 26, 27, 28]. These authors generalized the role of $($,$) and of the sources for BRST transformations and$ called them the antibracket and antifields respectively. Due to their contributions, this quantization procedure is often referred to as the Batalin-Vilkovisky formalism.

The antibracket formalism gained popularity among string theorists, when it was applied to the open bosonic string field theory [56, 246]. It has also proven quite useful for the closed string field theory and for topological field theories. Only within the last few years has it been applied to more general aspects of quantum field theory.

In some sense, the BRST approach, which was driven, in part, by renormalization considerations, and the field-antifield formalism, which was motivated by classical considerations such as gauge structure, are not so different. When sources are introduced for BRST transformations, the BRST approach resembles the field-antifield 
one. Antifields, then, have a simple intepretation: They are the sources for BRST transformations. In this sense, the field-antifield formalism is a general method for dealing with gauge theories within the context of standard field theory.

The general structure of the antibracket formalism is as follows. One introduces an antifield for each field and ghost, thereby doubling the total number of original fields. The antibracket $($,$) is an odd non-degenerate symplectic form on the space$ of fields and antifields. The original classical action $S_{0}$ is extended to a new action $S$, in an essentially unique way, to arrive at a theory with manifest BRST symmetry. One equation, the master equation $(S, S)=0$, reproduces in a compact way the gauge structure of the original theory governed by $S_{0}$. Although the master equation resembles the Zinn-Justin equation, the content of the two is different since $S$ is a functional of quantum fields and antifields and $\Gamma$ is a functional of classical fields.

The antibracket formalism currently appears to be the most powerful method for quantizing a gauge theory. Beyond tree level, order $\hbar$ terms usually need to be added to the action, thereby leading to a quantum action $W$. These counterterms are expected to render finite loop contributions, after a suitable regularization procedure has been introduced. The master equation must be appropriately generalized to the so-called quantum master equation. It involves a potentially singular operator $\Delta$. The regularization procedure and counterterms should also render $\Delta$ and its action on $W$ well-defined. Violations of the quantum master equation are equivalent to gauge anomalies [251]. To calculate correlation functions and scattering amplitudes in perturbation theory, a gauge-fixing procedure is selected. This procedure eliminates antifields in terms of functionals of fields. When appropriately implemented, propagators exist, and the usual Feynman graph methods can be used. In addition, for the study of symmetry properties, renormalization and anomalies, a modified version of the gauge-fixing procedure is available which keeps antifields. In short, the antibracket formalism has manifest gauge invariance or BRST symmetry, provides the extra fields needed for covariant quantization, permits a perturbative expansion of the quantum theory, and allows the study of quantum corrections to the symmetry structure of the theory.

The field-antifield formalism can treat systems that cannot be handled by FaddeevPopov functional integration approach. This is particularly clear for theories in which quartic ghost interactions arise [172, 81]. Faddeev-Popov quantization leads to an action bilinear in ghost fields, and fails for the case of open algebras. An open algebra occurs when the commutator of two gauge transformations produces a term proportional to the equations of motion and not just another gauge transformation [81, 27. In other words, the gauge algebra closes only on-shell. Such algebras occur in gravity [110] and supergravity [114, 172, 81, 258] theories. The ordinary Faddeev- 
Popov procedure also does not work for reducible theories. In reducible theories, the gauge generators are all not independent [67, 171, 6, 37, 228, 250, 260, 80, 242]. Some modifications of the procedure have been developed by introducing ghosts for ghosts [228, 178]. However, these modifications [228, 148, 178, 116] do not work for the general reducible theory. Even for Yang-Mills theories, the Faddeev-Popov procedure can fail, if one considers exotic gauge-fixing procedures for which "extraghosts" appear 172, 199, 200. The field-antifield formalism is sufficiently general to encompass previously known lagrangian approaches to the quantization of gauge theories.

Perhaps the most attractive feature of the field-antifield formalism is its imitation of a hamiltonian Poisson structure in a covariant way. In some instances, the hamiltonian approach to quantization has the advantage of being manifestly unitary. However, it is necessarily non-covariant since the time variable is treated in a manner different from the space variables. In addition, the gauge invariances usually must be fixed at the outset. In compensation for this, one needs to impose constraints on the Hilbert space of states. In the field-antifield approach, the antibracket plays the role of the Poisson bracket. As a consequence, hamiltonian concepts, such as canonical transformations, can be formulated and used [262, 263, 264, 27, 105, 251]. At the same time, manifest covariance and BRST invariance are maintained. Since the antibracket formalism proceeds via the functional integral, the powerful techniques of functional integration are available.

A non-trivial aspect of the field-antifield approach is the construction of the quantum action $W$. When loop effects are ignored, $W \rightarrow S$ provides the solution to the master equation. A straightforward but not necessarily simple procedure is available for obtaining $S$ given the classical action $S_{0}$ and its gauge invariances for a finitereducible system. When quantum effects are incorporated, $W$ must satisfy the more singular quantum master equation. However, there is currently no known method that guarantees the construction of $W$. The problem is that the field-antifield formalism does not automatically provide the functional integration measure. These issues are linked with those associated with unitarity, renormalization, quantum gauge invariance, and anomalies. Because these aspects of gauge theories are inherently difficult, it is not surprising that the field-antifield formalism does not provide a simple solution.

Another, less serious weakness, is that the antibracket formalism involves quite a bit of mathematical machinery. Sometimes, a gauge theory is expressed in a form which is more complicated than necessary. This can make computations somewhat more difficult.

The organization of this article is as follows. Sect. 2 discusses gauge structure. Some notation is presented during the process of introducing gauge transformations. 
The distinction between irreducible and reducible gauge theories is made. The latter involve a redundant set of gauge invariances so that there are relations among the gauge generators. As a result, there exists gauge invariances for gauge invariances, and ghosts for ghosts. A theory is Lth-stage reducible if there are gauge invariances for the gauge invariances for the gauge invariances, etc., $L$-fold times. The general form of the gauge structure for a first-stage reducible case is determined. In Sect. 3, specific gauge theories are presented to illustrate the concepts of Sect. 2. The spinless relativistic particle, non-abelian Yang-Mills theories, topological Yang-Mills theory, the antisymmetric tensor field, free abelian $p$-form theories, open bosonic string field theory, the massless relativistic spinning particle, and the first-quantized bosonic string are treated. The spinless relativistic particle of Sect. 3.1 is also used to exemplify notation. The massless relativistic spinning particle provides an example of a simple supergravity theory, namely a theory with supersymmetric gauge invariances. This system is used to illustrate the construction of supersymmetric and supergravity theories. A review of the construction of general-coordinate-invariant theories is given in the subsection on the first-quantized open bosonic string. These mini-reviews should be useful to the reader who is new to these subjects.

The key concepts of the field-antifield formalism are elucidated in Sect. 1 . Antifields are introduced and the antibracket is defined. The latter is used to define canonical transformations. They can be quite helpful in simplifying computations. Next, the classical master equation $(S, S)=0$ is presented. When appropriate boundary conditions are imposed, it reproduces, in a compact way, the gauge structure of Sect. 2. A suitable action $S$ satisfying the master equation is called a proper solution. Given the gauge-structure tensors of a first-stage reducible theory, Sect. 4.4 presents the generic proper solution. The last part of Sect. 国defines and discusses the classical BRST symmetry. Examples of proper solutions are provided in Sect. 5 for the gauge field theories presented in Sect. 色.

Sect. 6 begins the passage from the classical to the quantum aspects of the field-antifield formalism. The gauge-fixing procedure is discussed. The gauge-fixing fermion $\Psi$ is a key concept. It is used as a means of eliminating antifields in terms of functions of fields. The result is an action that is suitable for use in the path integral. Only in this context and in performing standard perturbative computations are antifields eliminated. It is shown that results are independent of the choice of $\Psi$, if the quantum action $W$ satisfies the quantum master equation. To implement gauge-fixing, more fields and their antifields must be introduced. How this works for irreducible and first-stage reducible theories is treated first. Then, for reference purposes, the general $L$ th-stage reducible case is considered. Delta-function type gauge-fixing is treated in Sect. 6.3. Again, irreducible and first-stage reducible cases 
are presented first. Again, for reference purposes, the general Lth-stage reducible case is treated. Gauge-fixing by a gaussian averaging process is discussed in Sect. 6.4. After gauge-fixing, a classical gauge-fixed BRST symmetry can be defined. See Sect. 6.5. The freedom to perform canonical transformations permits one to work in any appropriate field basis. This freedom can be quite useful. Concepts tend to have different interpretations in different bases. One basis, associated with $\Psi$ and called the gauge-fixed basis, is the last topic of Sect. 6. Examples of gauge-fixing procedures are provided in Sect. 7. With the exception of the free $p$-form theory, the theories are the ones considered in Sects. 3 and 5 .

Quantum effects and possible gauge anomalies are analyzed in Sect. 8. The key concepts are quantum-BRST transformations and the quantum master equation. Techniques for assisting in finding solutions to the quantum master equation are provided in Sects. 8.2, 8.5 and 8.6. The generating functional $\Gamma$ for one-particleirreducible diagrams is generalized to the field-antifield case in Sect. 8.4. This allows one to treat the quantum system in a manner similar to the classical system. The Zinn-Justin equation is shown to be equivalent to the quantum master equation. When unavoidable violations of the latter occur, the gauge theory is anomalous. See Sect. 8.5. Explicit formulas at the one-loop level are given. In Sect. 9, sample anomaly calculations are presented. It is shown that the spinless relativistic particle does not have an anomaly. In Sect. 9.2, the field-antifield treatment of the two-dimensional chiral Schwinger model is presented. Violations of the quantum master equation are obtained. This is expected since the theory is anomalous. A similar computation is performed for the open bosonic string. For $D \neq 26$, the theory is anomalous, as expected. Some of the details of the calculations are relegated to Appendix C.

Section 10 briefly presents several additional topics. The application of the fieldantifield formalism to global symmetries is presented. A review is given of the geometric interpretation of E. Witten [273]. The next topic is the role of locality. This somewhat technical issue is important for renormalizability and for cohomological aspects. A summary of cohomological methods is given. Next, the relation between the hamiltonian and antibracket approaches is discussed. The question of unitarity is the subject of Sect. 10.6. One place where the field-antifield formalism has played an essential role is in the $D=26$ closed bosonic string field theory. This example is rather complicated and not suitable for pedagogical purposes. Nevertheless, general aspects of the antibracket formalism for the closed string field theory are discussed. Finally, an overview is given of how to handle anomalous systems using an extended set of fields and antifields.

Appendix A reviews the mathematical aspects of left and right derivatives, integration by parts, and chain rules for differentiation. Appendix B discusses in more 
detail the regularity condition, which is a technical requirement of the antibracket formalism.

At every stage of development of the formalism, there exists some type of BRST operator. In the space of fields and antifields before quantization, a classical nilpotent BRST transformation $\delta_{B}$ is defined by using the action $S$ and the antibracket: $\delta_{B} F=(F, S)$. From $\delta_{B}$, a gauge-fixed version $\delta_{B \Psi}$ is obtained by imposing the conditions on antifields provided by the gauge-fixing fermion $\Psi$. At the quantum level, a quantum version $\delta_{\hat{B}}$ of $\delta_{B}$ emerges. In the context of the effective action formulation, a transformation $\delta_{B_{c q}}$, acting on classical fields, can be defined by using $\Gamma$ in lieu of $S$. Several subsections are devoted to the BRST operator, its properties and its utility.

The existence of a BRST symmetry is crucial to the development. Observables are those functionals which are BRST invariant and cannot be expressed as the BRST variation of something else. In other words, observables correspond to the elements of the BRST cohomology. The nilpotency of $\delta_{B}$ and $\delta_{\hat{B}}$ are respectively equivalent to the classical and quantum master equations. The traditional treatment of gauge theories using BRST invariance is reviewed in [32]. For this reason, we do not discuss BRST quantization in detail.

The antibracket formalism is rather versatile in that one can use any set of fields (and antifields) related to the original fields (and antifields) by a canonical transformation. However, under such a change, the meaning of certain concepts change. For example, the gauge structure, as determined by the master equation, has a different interpretation in the gauge-fixed basis than in the original basis. Most of this review uses the second viewpoint. The treatment in the gauge-fixed basis is handled in Sects. 6.6 and 8.4 .

The material in each section strives to fulfill one of three purposes. A key purpose is to present computations that lead to understanding and insight. Sections 2, 6, 6.1, 6.5, 6.6, 8 and 10 are mainly of this character. The second purpose is pedagogical. This Introduction falls into this category in that it gives a quick overview of the formalism and the important concepts. Sections 3 , 5, 0, and 9 analyze specially chosen gauge theories which allow the reader to understand the field-antifield formalism in a concrete manner. Finally, some material is included for technical completeness. Sections 6.2 6.4 present methods for gauge-fixing the generic gauge theory. Parts of sections 2.2, 2.4 and 8.7 are also for reference purposes. Probably the reader should not initially try to read these sections in detail. Many sections serve a dual role.

A few new results on the antibracket formalism are presented in this review. They are included because they provide insight for the reader. We have tried to have a minimum overlap with other reviews. In particular, cohomological aspects are covered in [36, 89, 57, 32, 93, 8, 152, 157, 253] and more-detailed aspects of anomalies 
are treated in [253]. Pedagogical treatments are given in references [157, 253]. In certain places, material from reference [206] has been used.

This review focuses on the key points and concepts of antibracket formalism. There is some emphasis on applications to string theory. Our format is to first present the material abstractly and then to supply examples. The reader who is new to this subject and mainly interested in learning may wish to reverse this order. Exercises can be generated by verifying the abstract results in each of the sample gauge theories of Sects. 3, 5, 7, and 9. Other systems, which have been treated by field-antifield quantization and may be of use to the reader, are the free spin $\frac{5}{2}$ field [26], the spinning string [136], the 10-dimensional Brink-Schwarz superparticle and superstring [123, 139, 173, [90, 211, 43, 232, 44, 227], chiral gravity [77, $W_{3}$ gravity [161, 45, 74, 162, 72, 257, general topological field theories [66, 185, 50, 49, 127, 164, 191, 158, 79], the supersymmetric Wess-Zumino model [33, 159] and chiral gauge theories in four-dimensions [251. The antibracket formalism has found various interpretations in mathematics [125, 126, 127, 209, 189, 198, 218, 219, 237]. Some other recent relevant work can be found in [261, 21, 22, 47, 147]. The referencing in this review is thorough but not complete. A restriction has been made to only cite works directly relevant to the issues addressed in each section. Multiple references are done first chronologically and then alphabetically. The titles of references are provided to give the reader a better indication of the content of each work.

We work in Minkowski space throughout this article. Functional integrals are defined by analytic continuation using Wick rotation. This is illustrated in the computations of Appendix C. We use $\eta_{\mu \nu}$ to denote the flat-space metric with the signature convention $(-1,1,1, \ldots, 1)$. Flat-space indices are raised and lowered with this metric. The epsilon tensor $\varepsilon_{\mu_{0} \mu_{1} \mu_{2} \ldots \mu_{d-1}}$ is determined by the requirement that it be antisymmetric in all indices and that $\varepsilon_{012 \ldots d-1}=1$, where $d-1$ and $d$ are respectively the dimension of space and space-time. We often use square brackets to indicate a functional of fields and antifields to avoid confusion with the antibracket, i.e., $S\left[\Phi, \Phi^{*}\right]$ in lieu of $S\left(\Phi, \Phi^{*}\right)$.

\footnotetext{
${ }^{1}$ See [227 for additional references.
} 


\section{Structure of the Set of Gauge Transformations}

The most familiar example of a gauge structure is the one associated with a non-abelian Yang-Mills theory [276], namely a Lie group. The commutator of two Lie-algebra generators produces a Lie-algebra generator. When a basis is used, this commutator algebra is determined by the structure constants of the Lie group. For example, for the Lie algebra $s u(2)$ there are three generators and the structure constant is the anti-symmetric tensor on three indices $\varepsilon^{\alpha \beta \gamma}$. A commutator algebra, as determined by a set of abstract structure constants, does not necessarily lead to a Lie algebra. The Jacobi identity, which expresses the associativity of the algebra, must be satisfied [258].

Sometimes, in more complicated field theories, the transformation rules involve field-dependent structure constants. Such cases are sometimes referred to as "soft algebras" [17, 236]. In such a situation, the determination of the gauge algebra is more complicated than in the Yang-Mills case. The Jacobi identity must be appropriately generalized [17, 24, 84]. Furthermore, new structure tensors beyond commutator structure constants may appear and new identities need to be satisfied.

In other types of theories, the generators of gauge transformations are not independent. This occurs when there is "a gauge invariance" for gauge transformations. One says the system is reducible. A simple example is a theory constructed using a three-form $F$ which is expressed in terms of a two-form $B$ by applying the exterior derivative $F=d B$. The gauge invariances are given by the transformation rule $\delta B=d A$ for any one-form $A$. The theory is invariant under such transformations because the lagrangian is a functional of $F$ and $F$ is invariant: $\delta F=d \delta B=d d A=0$. However, the gauge invariances are not all independent since modifying $A$ by $\delta A=d \lambda$ for some zero-form $\lambda$ leads to no change in the transformation for $B$. When $A=d \lambda$, $\delta B=d A=d d \lambda=0$. The structure of a gauge theory is more complicated than the Yang-Mills case when there are gauge invariances for gauge transformations.

Another complication occurs when the commutator of two gauge transformations produces a term that vanishes on-shell, i.e., when the equations of motion are used. When equations of motion appear in the gauge algebra, how should one proceed?

In this section we discuss the above-mentioned complications for a generic gauge theory. The questions are (i) what are the relevant gauge-structure tensors and (ii) what equations do they need to satisfy. The answers to these questions lead us to the gauge structure of a theory.

This section constitutes a somewhat technical but necessary prelude. A reader

might want to consult the examples in Sect. 3. The more interesting development of the field-antifield formalism begins in Sect. 4 . 


\subsection{Gauge Transformations}

This subsection introduces the notions of a gauge theory and a gauge transformation. It also defines notation. The antibracket approach employs an elaborate mathematical formalism. Hence, one should try to become quickly familiar with notation and conventions.

Consider a system whose dynamics is governed by a classical action $S_{0}[\phi]$, which depends on $n$ different fields $\phi^{i}(x), i=1, \cdots, n$. The index $i$ can label space-time indices $\mu, \nu$ of tensor fields, the spinor indices of fermion fields, and/or an index distinguishing different types of generic fields. At the classical level, the fields are functions of space-time. In the quantum system, they are promoted to operators. In this section, we treat the classical case only.

Let $\epsilon\left(\phi^{i}\right)=\epsilon_{i}$ denote the statistical parity of $\phi^{i}$. Each $\phi^{i}$ is either a commuting field $\left(\epsilon_{i}=0\right)$ or an anticommuting field $\left(\epsilon_{i}=1\right)$. One has $\phi^{i}(x) \phi^{j}(y)=(-1)^{\epsilon_{i} \epsilon_{j}} \phi^{j}(y) \phi^{i}(x)$.

Let us assume that the action is invariant under a set of $m_{0}\left(m_{0} \leq n\right)$ non-trivial gauge transformations, which, when written in infinitesimal form, read

$$
\delta \phi^{i}(x)=\left(R_{\alpha}^{i}(\phi) \varepsilon^{\alpha}\right)(x) \quad, \quad \text { where } \alpha=1 \text { or } 2 \ldots \text { or } m_{0}
$$

Here, $\varepsilon^{\alpha}(x)$ are infinitesimal gauge parameters, that is, arbitrary functions of the space-time variable $x$, and $R_{\alpha}^{i}$ are the generators of gauge transformations. These generators are operators that act on the gauge parameters. In kernel form, $\left(R_{\alpha}^{i}(\phi) \varepsilon^{\alpha}\right)(x)$ can be represented as $\int \mathrm{d} y R_{\alpha}^{i}(x, y) \varepsilon^{\alpha}(y)$.

It is convenient to adopt the following compact notation [82, 83]. Unless otherwise stated, the appearance of a discrete index also indicates the presence of a spacetime variable. We then use a generalized summation convention in which a repeated discrete index implies not only a sum over that index but also an integration over the corresponding space-time variable. As a simple example, consider the multiplication of two matrices $g$ and $h$, written with explicit matrix indices. In compact notation,

$$
f_{B}^{A}=g_{C}^{A} h_{B}^{C}
$$

becomes not only a matrix product in index space but also in function space. Eq.(2.2) represents

$$
f^{A}{ }_{B}(x, y)=\sum_{C} \int \mathrm{d} z g_{C}^{A}(x, z) h_{B}^{C}(z, y)
$$

in conventional notation. In other words, the index $A$ in Eq.(2.2) stands for $A$ and $x$ in Eq.(2.3). Likewise, $B$ and $C$ in Eq.(2.2) represent $\{B, y\}$ and $\{C, z\}$. The generalized summation convention for $C$ in compact notation yields a sum over the discrete index $C$ and an integration over $z$ in conventional notation in Eq.(2.3). The 
indices $A, B$ and $C$ in compact notation implicitly represent space-time variables $x$, $y, z$, etc., and explicitly can be field indices $i, j, k$, etc., gauge index $\alpha, \beta, \gamma$, etc., or any other discrete index in the formalism.

With this convention, the transformation laws

$$
\delta \phi^{i}(x)=\sum_{\alpha} \int \mathrm{d} y R_{\alpha}^{i}(x, y) \varepsilon^{\alpha}(y)
$$

can be written succinctly as

$$
\delta \phi^{i}=R_{\alpha}^{i} \varepsilon^{\alpha} .
$$

The index $\alpha$ in Eq.(2.5) corresponds to the indices $y$ and $\alpha$ in Eq.(2.4). The index $i$ in Eq.(2.5) corresponds to the indices $x$ and $i$ in Eq.(2.4). The compact notation is illustrated in the example of Sect. 3.1. Although this notation might seem confusing at first, it is used extensively in the antibracket formalism. In the next few paragraphs, we present equations in both notations.

Each gauge parameter $\varepsilon^{\alpha}$ is either commuting, $\epsilon\left(\varepsilon^{\alpha}\right) \equiv \epsilon_{\alpha}=0$, or is anticommuting, $\epsilon_{\alpha}=1$. The former case corresponds to an ordinary symmetry while the latter is a supersymmetry. The statistical parity of $R_{\alpha}^{i}, \epsilon\left(R_{\alpha}^{i}\right)$, is determined from Eq.(2.1): $\epsilon\left(R_{\alpha}^{i}\right)=\left(\epsilon_{i}+\epsilon_{\alpha}\right) \quad(\bmod 2)$.

Let $S_{0, i}(\phi, x)$ denote the variation of the action with respect to $\phi^{i}(x)$ :

$$
S_{0, i}(\phi, x) \equiv \frac{\partial_{r} S_{0}[\phi]}{\partial \phi^{i}(x)}
$$

where the subscript $r$ indicates that the derivative is to be taken from the right (see Appendix A). Henceforth, when a subscript index $i, j$, etc., appears after a comma it denotes the right derivative with respect to the corresponding field $\phi^{i}, \phi^{j}$, etc.. In compact notation, we write Eq.(2.6) as $S_{0, i}=\frac{\partial_{r} S_{0}}{\partial \phi^{i}}$ where the index $i$ here stands for both $x$ and $i$ in Eq.(2.6).

The statement that the action is invariant under the gauge transformation in Eq.(2.1) means that the Noether identities

$$
\int \mathrm{d} x \sum_{i=1}^{n} S_{0, i}(x) R_{\alpha}^{i}(x, y)=0
$$

hold, or equivalently, in compact notation

$$
S_{0, i} R_{\alpha}^{i}=0
$$

Eq.(2.8) is derived by varying $S_{0}$ with respect to right variations of the $\phi^{i}$ given by Eq.(2.1). When using right derivatives, the variation $\delta S_{0}$ of $S_{0}$, or of any other object, is given by $\delta S_{0}=S_{0, i} \delta \phi^{i}$. If one were to use left derivatives, the variation 
of $S_{0}$ would read $\delta S_{0}=\delta \phi^{i} \frac{\partial_{l} S_{0}}{\partial \phi^{i}}$. Eq.(2.7) is sometimes zero because the integrand is a total derivative. We assume that surface terms can be dropped in such integrals - this is indeed the case when Eq.(2.7) is applied to gauge parameters that fall off sufficiently fast at spatial and temporal infinity. The Noether identities in Eq.(2.8) are the key equations of this subsection and can even be thought of as the definition of when a theory is invariant under a gauge transformation of the form in Eq.(2.1).

To commence perturbation theory, one searches for solutions to the classical equations of motion, $S_{0, i}(\phi, x)=0$, and then expands about these solutions. We assume there exists at least one such stationary point $\phi_{0}=\left\{\phi_{0}^{j}\right\}$ so that

$$
\left.S_{0, i}\right|_{\phi_{0}}=0
$$

Equation (2.9) defines a surface $\Sigma$ in function space, which is infinite dimensional when gauge symmetries are present.

As a consequence of the Noether identities, the equations of motion are not independent. Furthermore, new saddle point solutions can be obtained by performing gauge transformations on any particular solution. These new solutions should not be regarded as representing new physics however - fields related by local gauge transformations are considered equivalent.

The Noether identities also imply that propagators do not exist. By differentiating the identities from the left with respect $\phi^{j}$, one obtains

$$
\begin{aligned}
\frac{\partial_{l}}{\partial \phi^{i}}\left(S_{0, j} R_{\alpha}^{j}\right) & =\left(\frac{\partial_{l} \partial_{r} S_{0}}{\partial \phi^{i} \partial \phi^{j}}\right) R_{\alpha}^{j}+S_{0, j} \frac{\partial_{l} R_{\alpha}^{j}}{\partial \phi^{i}}(-1)^{\epsilon_{i} \epsilon_{j}}=0 \\
& \left.\Rightarrow\left(\frac{\partial_{l} \partial_{r} S_{0}}{\partial \phi^{i} \partial \phi^{j}}\right) R_{\alpha}^{j}\right|_{\phi_{0}}=0,
\end{aligned}
$$

i.e., the hessian $\left(\frac{\partial_{l} \partial_{r} S_{0}}{\partial \phi^{i} \partial \phi^{j}}\right)$ of $S_{0}$ is degenerate at any point on the stationary surface $\Sigma$. The $R_{\alpha}^{i}$ are on-shell null vectors of this hessian. Since propagators involve the inverse of this hessian, propagators do not exist for certain combinations of fields. This means that the standard loop expansion cannot be straightforwardly applied. A method is required to overcome this problem.

Technically speaking, to study the structure of the set of gauge transformations it is necessary to assume certain regularity conditions on the space for which the equations of motion $S_{0, i}=0$ hold. The interested reader can find these conditions in Appendix B. A key consequence of the regularity conditions is that if a function $F(\phi)$ of the fields $\phi$ vanishes on-shell, that is, when the equations of motion are implemented, then $F$ must be a linear combination of the equations of motion, i.e.,

$$
\left.F(\phi)\right|_{\Sigma}=0 \Rightarrow F(\phi)=S_{0, i} \lambda^{i}(\phi)
$$


where $\left.\right|_{\Sigma}$ indicates the restriction to the surface where the equations of motion hold [81, 28, 106, 103, 105. Eq.(2.11) can be thought of as a completeness relation for the equations of motion. We shall make use of Eq.(2.11) frequently.

Throughout Sect. 2, we assume that the gauge generators are fixed once and for all. One could take linear combinations of the generators to form a new set. This would change the gauge-structure tensors presented below. This non-uniqueness is not essential and is discussed in Sect. 4.5.

To see explicit examples of the abstract formalism that follows, one may want to glance from time to time at the examples of Sect. 0 .

\subsection{Irreducible and Reducible Gauge Theories}

It is important to know any dependences among the gauge generators. Only with this knowledge is possible to determine the independent degrees of freedom. The purpose of this subsection is to analyze this issue in more detail for the generic case.

The simplest gauge theories, for which all gauge transformations are independent, are called irreducible. When dependences exist, the theory is reducible. In reducible gauge theories, there is a "kind of gauge invariance for gauge transformations" or what one might call "level-one" gauge invariances. If the level-one gauge transformations are independent, then the theory is called first-stage reducible. This may not happen. Then, there are "level-two" gauge invariances, i.e., gauge invariances for the level-one gauge invariances and so on. This leads to the concept of an $L$-th stage reducible theory. In what follows we let $m_{s}$ denote the number of gauge generators at the $s$-th stage regardless of whether they are independent.

Let us define more precisely the above concepts. Assume that all gauge invariances of a theory are known and that the regularity condition described in Appendix B is satisfied. Then, the most general solution to the Noether identities (2.8) is a gauge transformation, up to terms proportional to the equations of motion:

$$
S_{0, i} \lambda^{i}=0 \Leftrightarrow \lambda^{i}=R_{0 \alpha_{0}}^{i} \lambda^{\prime \alpha_{0}}+S_{0, j} T^{j i},
$$

where $T^{i j}$ must satisfy the graded symmetry property

$$
T^{i j}=-(-1)^{\epsilon_{i} \epsilon_{j}} T^{j i} .
$$

The $R_{0 \alpha_{0}}^{i}$ are the gauge generators in Eq.(2.1). For notational convenience, we have appended a subscript 0 on the gauge generator and the gauge index $\alpha$. This subscript indicates the level of the gauge transformation. The second term $S_{0, j} T^{j i}$ in Eq.(2.12) is known as a trivial gauge transformation. Such transformations are discussed in the next subsection. It is easily checked that the action is invariant under such 
transformations due to the trivial commuting or anticommuting properties of the $S_{0, j}$. The first term $R_{0 \alpha_{0}}^{i} \lambda^{\prime \alpha_{0}}$ in Eq. (2.12) is similar to a non-trivial gauge transformation of the form of Eq.(2.1) with $\varepsilon^{\alpha_{0}}=\lambda^{\prime \alpha_{0}}$. The key assumption in Eq.(2.12) is that the set of functionals $R_{0 \alpha_{0}}^{i}$ exhausts on-shell the relations among the equations of motion, namely the Noether identities. In other words, the gauge generators are on-shell a complete set. This is essentially equivalent to the regularity condition.

If the functionals $R_{0 \alpha_{0}}^{i}$ are independent on-shell then the theory is irreducible. In such a case,

$$
\left.\operatorname{rank} R_{0 \alpha_{0}}^{i}\right|_{\Sigma}=m_{0}
$$

where $m_{0}$ is the number of gauge transformations. The rank of the hessian

$$
\left.\operatorname{rank}\left(\frac{\partial_{l} \partial_{r} S_{0}}{\partial \phi^{i} \partial \phi^{j}}\right)\right|_{\Sigma}=n-\left.\operatorname{rank} R_{\alpha}^{i}\right|_{\Sigma}
$$

is $n-m_{0}$. Define the net number of degrees of freedom $n_{\text {dof }}$ to be the number of fields that enter dynamically in $S_{0}$, regardless of whether they propagate. 2 Then for an irreducible theory $n_{\text {dof }}$ is $n-m_{0}$ since there are $m_{0}$ gauge degrees of freedom. Note that $n_{\text {dof }}$ matches the rank of the hessian in Eq.(2.15).

If, however, there are dependences among the gauge generators, and the rank of the generators is less than their number, $\left.\operatorname{rank} R_{0 \alpha_{0}}^{i}\right|_{\Sigma}<m_{0}$, then the theory is reducible. If $m_{0}-m_{1}$ of the generators are independent on-shell, then there are $m_{1}$ relations among them and there exist $m_{1}$ functionals $R_{1 \alpha_{1}}^{\alpha_{0}}$ such that

$$
\begin{aligned}
R_{0 \alpha_{0}}^{i} R_{1 \alpha_{1}}^{\alpha_{0}} & =S_{0, j} V_{1 \alpha_{1}}^{j i}, \quad \alpha_{1}=1, \ldots, m_{1}, \\
\epsilon\left(R_{1 \alpha_{1}}^{\alpha_{0}}\right) & =\epsilon_{\alpha_{0}}+\epsilon_{\alpha_{1}}(\bmod 2),
\end{aligned}
$$

for some $V_{1 \alpha_{1}}^{j i}$, satisfying $V_{1 \alpha_{1}}^{i j}=-(-1)^{\epsilon_{i} \epsilon_{j}} V_{1 \alpha_{1}}^{j i}$. Here, $\epsilon_{\alpha_{1}}$ is the statistical parity of the level-one gauge parameter. The $R_{1 \alpha_{1}}^{\alpha_{0}}$ are the on-shell null vectors for $R_{0 \alpha_{0}}^{i}$ since $\left.R_{0 \alpha_{0}}^{i} R_{1 \alpha_{1}}^{\alpha_{0}}\right|_{\Sigma}=0$. The presence of $V_{1 \alpha_{1}}^{j i}$ in Eq.(2.16) is a way of extending this statement off-shell. Here and elsewhere, when a combination of field equations appears on the right-hand side of an equation, it indicates the off-shell extension of an on-shell statement; such an extension can be performed using the regularity postulate of Appendix B. Note that, if $\varepsilon^{\alpha}=R_{1 \alpha_{1}}^{\alpha} \varepsilon_{1}^{\alpha}$ for any $\varepsilon_{1}^{\alpha}$, then $\delta \phi^{i}$ in Eq.(2.5) is zero onshell, so that no gauge transformation is produced. In Eq.(2.16) it is assumed that the reducibility of the $R_{0 \alpha_{0}}^{i}$ is completely contained in $R_{1 \alpha_{1}}^{\alpha_{0}}$, i.e., $R_{1 \alpha_{1}}^{\alpha_{0}}$ also constitute a complete set

$$
R_{0 \alpha_{0}}^{i} \lambda^{\alpha_{0}}=S_{0, j} M_{0}^{j i} \Rightarrow \lambda^{\alpha_{0}}=R_{1 \alpha_{1}}^{\alpha_{0}} \lambda^{\prime \alpha_{1}}+S_{0, j} T_{0}^{j \alpha_{0}}
$$

${ }^{2}$ In electromagnetism, $n_{\text {dof }}=3$, but there are only two propagating degrees of freedom corresponding to the two physical polarizations. 
for some $\lambda^{\prime \alpha_{1}}$ and some $T_{0}^{j \alpha_{0}}$.

If the functionals $R_{1 \alpha_{1}}^{\alpha_{0}}$ are independent on-shell

$$
\operatorname{rank} R_{\left.1 \alpha_{1}\right|_{\Sigma}}^{\alpha_{0}}=m_{1}
$$

then the theory is called first-stage reducible. One also has rank $\left.R_{0 \alpha_{0}}^{i}\right|_{\Sigma}=m_{0}-m_{1}$ and the net number of degrees of freedom in the theory is $n-m_{0}+m_{1}$. Since true and gauge degrees of freedom have been determined,

$$
\left.\operatorname{rank} \frac{\partial_{l} \partial_{r} S_{0}}{\partial \phi^{i} \partial \phi^{j}}\right|_{\Sigma}=n-m_{0}+m_{1}
$$

If the functionals $R_{1 \alpha_{1}}^{\alpha_{0}}$ are not all independent on-shell, relations exist among them and the theory is second-or-higher-stage reducible. Then, the on-shell null vectors of $R_{1 \alpha_{1}}^{\alpha_{0}}$ and higher $R$-type tensors must be found.

One continues the above construction until it terminates. A theory is L-th stage reducible [26] if there exist functionals

$$
R_{s \alpha_{s}}^{\alpha_{s-1}}, \quad \alpha_{s}=1, \ldots, m_{s}, \quad s=0, \ldots, L
$$

such that $R_{0 \alpha_{0}}^{i}$ satisfies Eq.(2.8), i.e., $S_{0, i} R_{0 \alpha_{0}}^{i}=0$, and such that, at each stage, the $R_{s \alpha_{s}}^{\alpha_{s-1}}$ constitute a complete set, i.e.,

$$
\begin{gathered}
R_{s \alpha_{s}}^{\alpha_{s-1}} \lambda^{\alpha_{s}}=S_{0, j} M_{s}^{j \alpha_{s-1}} \Rightarrow \lambda^{\alpha_{s}}=R_{s+1, \alpha_{s+1}}^{\alpha_{s}} \lambda^{\prime \alpha_{s+1}}+S_{0, j} T_{s}^{j \alpha_{s}}, \\
R_{s-1, \alpha_{s-1}}^{\alpha_{s-2}} R_{s \alpha_{s}}^{\alpha_{s-1}}=S_{0, i} V_{s \alpha_{s}}^{i \alpha_{s-2}}, \quad s=1, \ldots, L, \\
\left.\operatorname{rank} R_{s \alpha_{s}}^{\alpha_{s-1}}\right|_{\Sigma}=\sum_{t=s}^{L}(-1)^{t-s} m_{t}, \quad s=0, \ldots, L,
\end{gathered}
$$

where we have defined $R_{0 \alpha_{0}}^{\alpha_{-1}} \equiv R_{0 \alpha_{0}}^{i}$ and $\alpha_{-1} \equiv i$. The $R_{s \alpha_{s}}^{\alpha_{s-1}}$ are the on-shell null vectors for $R_{s-1 \alpha_{s-1}}^{\alpha_{s-2}}$. The statistical parity of $R_{s \alpha_{s}}^{\alpha_{s-1}}$ is $\left(\epsilon_{\alpha_{s-1}}+\epsilon_{\alpha_{s}}\right)(\bmod 2)$, where $\epsilon_{\alpha_{s}}$ is the statistical parity of the $s$-level gauge transformation associated with the index $\alpha_{s}$. Finally,

$$
n_{\mathrm{dof}}=\left.\operatorname{rank} \frac{\partial_{l} \partial_{r} S_{0}}{\partial \phi^{i} \partial \phi^{j}}\right|_{\Sigma}=n-\sum_{s=0}^{L}(-1)^{s} m_{s}
$$

is the net number of degrees of freedom. 


\subsection{Trivial Gauge Transformations}

As mentioned in the last subsection, trivial gauge transformations exist. Since they are proportional to the equations of motion they do not lead to conservation laws. This subsection discusses their role in the gauge algebra.

Given that the finite invertible gauge transformations satisfy the group axioms, their infinitesimal counterparts necessarily form an algebra. Besides the usual gauge transformations (2.1), there are the trivial transformations, defined as

$$
\delta_{\mu} \phi^{i}=S_{0, j} \mu^{j i}, \quad \mu^{j i}=-(-1)^{\epsilon_{i} \epsilon_{j}} \mu^{i j},
$$

where $\mu^{j i}$ are arbitrary functions. It is easily demonstrated that, as a consequence of the symmetry properties of $\mu^{j i}$, the transformations in Eq.(2.21) leave the action invariant. In studying the structure of the gauge transformations, it is necessary to take into consideration the presence of such transformations.

To determine their effect on the gauge algebra, consider the commutator of a trivial transformation with any other transformation. Calling the latter $\delta_{r} \phi^{i}=r^{i}$, one has

$$
\left[\delta_{\mu}, \delta_{r}\right] \phi^{i}=r_{, k}^{i} S_{0, j} \mu^{j k}-S_{0, j} \mu_{, k}^{j i} r^{k}-S_{0, j k} r^{k} \mu^{j i}
$$

Given that $\delta_{r}$ is a symmetry transformation of $S_{0}$, it follows by differentiation by $\phi^{j}$ that

$$
S_{0, k} r^{k}=0 \Rightarrow S_{0, j k} r^{k}+S_{0, k} r_{, j}^{k}=0 \quad,
$$

so that the commutator becomes

$$
\left[\delta_{\mu}, \delta_{r}\right] \phi^{i}=S_{0, j}\left(r_{, k}^{j} \mu^{k i}-(-1)^{\epsilon_{i} \epsilon_{j}} r_{, k}^{i} \mu^{k j}-\mu_{, k}^{j i} r^{k}\right)=S_{0, j} \tilde{\mu}^{j i}
$$

from which one concludes that the commutator of a trivial transformation with any other transformation is a trivial transformation. Hence, the trivial transformations are a normal subgroup $H$ of the full group of gauge transformations, $\bar{G}$.

The trivial gauge transformations are of no physical significance: They neither lead to conserved currents nor do they prevent the development of a perturbative expansion about a stationary point. They are simply a consequence of having more than one degree of freedom. On these grounds, it would seem sensible to dispense with them and restrict oneself to the quotient $G=\bar{G} / H$. However, this is only possible in certain cases. In general, the commutator of two non-trivial gauge transformations produces trivial gauge transformations. Furthermore, for reasons of convenience, particularly when it is desirable to have manifest covariance or preserve locality, one sometimes wants to include trivial transformations. Hence, the full group $\bar{G}$ is used for studying the gauge structure of the theory. 


\subsection{The Gauge Structure}

In this section we restrict ourselves to the simpler cases of irreducible and firststage-reducible gauge theories. To avoid cumbersome notation, we use $R_{\alpha}^{i}$ for $R_{0 \alpha_{0}}^{i}$, $Z_{a}^{\alpha}$ for $R_{1 \alpha_{1}}^{\alpha_{0}}$, and in Eq.(2.16) we use $V_{a}^{j i}$ for $V_{1 \alpha_{1}}^{j i}$, so that the indices $\alpha_{0}$ and $\alpha_{1}$ respectively correspond to $\alpha$ and $a$.

The general strategy in obtaining the gauge structure is as follows [81]. The first gauge-structure tensors are the gauge generators themselves, and the first gaugestructure equations are the Noether identities (2.8). One computes commutators, commutators of commutators, etc., of gauge transformations. Graded symmetrization produces identity equations for the structure tensors that must be satisfied. Generic solutions are obtained by exploiting the consequences of the regularity conditions, namely, completeness. In using completeness, additional gauge-structure tensors appear. They enter in higher-order symmetrized commutator identity equations. The process is continued until it terminates.

Although this section provides some insight, it is somewhat technical so that the reader may wish to skip it at first. If one is only interested in the irreducible case, one should read to Eq.(2.36). For reasons of space, many details of the algebra are omitted. As an exercise, the reader can provide the missing steps.

Consider the commutator of two gauge transformations of the type in Eq.(2.1). On one hand, a direct computation leads to

$$
\left[\delta_{1}, \delta_{2}\right] \phi^{i}=\left(R_{\alpha, j}^{i} R_{\beta}^{j}-(-1)^{\epsilon_{\alpha} \epsilon_{\beta}} R_{\beta, j}^{i} R_{\alpha}^{j}\right) \varepsilon_{1}^{\beta} \varepsilon_{2}^{\alpha}
$$

On the other hand, since this commutator is also a gauge symmetry of the action it satisfies the Noether identity so that, factoring out the gauge parameters $\varepsilon_{1}^{\beta}$ and $\varepsilon_{2}^{\alpha}$, one may write

$$
S_{0, i}\left(R_{\alpha, j}^{i} R_{\beta}^{j}-(-1)^{\epsilon_{\alpha} \epsilon_{\beta}} R_{\beta, j}^{i} R_{\alpha}^{j}\right)=0
$$

Taking into account Eq.(2.12) the above equation implies the following important relation among the generators

$$
R_{\alpha, j}^{i} R_{\beta}^{j}-(-1)^{\epsilon_{\alpha} \epsilon_{\beta}} R_{\beta, j}^{i} R_{\alpha}^{j}=R_{\gamma}^{i} T_{\alpha \beta}^{\gamma}-S_{0, j} E_{\alpha \beta}^{j i}
$$

for some gauge-structure tensors $T_{\alpha \beta}^{\gamma}$ and $E_{\alpha \beta}^{j i}$. This equation defines $T_{\alpha \beta}^{\gamma}$ and $E_{\alpha \beta}^{j i}$. Restoring the dependence on the gauge parameters $\varepsilon_{1}^{\beta}$ and $\varepsilon_{2}^{\alpha}$, the last two equations imply

$$
\left[\delta_{1}, \delta_{2}\right] \phi^{i} \equiv R_{\gamma}^{i} T_{\alpha \beta}^{\gamma} \varepsilon_{1}^{\beta} \varepsilon_{2}^{\alpha}-S_{0, j} E_{\alpha \beta}^{j i} \varepsilon_{1}^{\beta} \varepsilon_{2}^{\alpha} \quad,
$$

where $T_{\alpha \beta}^{\gamma}$ are known as the "structure constants" of the gauge algebra. The words structure constants are in quotes because in general the $T_{\alpha \beta}^{\gamma}$ depend on the fields of 
the theory and are not "constant". The possible presence of the $E_{\alpha \beta}^{j i}$ term is due to the fact that the commutator of two gauge transformations may give rise to trivial gauge transformations [81, 24, 27].

The gauge algebra generated by the $R_{\alpha}^{i}$ is said to be open if $E_{\alpha \beta}^{i j} \neq 0$, whereas the algebra is said to be closed if $E_{\alpha \beta}^{i j}=0$. Moreover, Eq.(2.22) defines a Lie algebra if the algebra is closed, $E_{\alpha \beta}^{i j}=0$, and the $T_{\alpha \beta}^{\gamma}$ do not depend on the fields $\phi^{i}$.

The gauge-structure tensors have the following symmetry properties under the interchange of indices

$$
\begin{gathered}
E_{\alpha \beta}^{i j}=-(-1)^{\epsilon_{i} \epsilon_{j}} E_{\alpha \beta}^{j i}=-(-1)^{\epsilon_{\alpha} \epsilon_{\beta}} E_{\beta \alpha}^{i j}, \\
T_{\alpha \beta}^{\gamma}=-(-1)^{\epsilon_{\alpha} \epsilon_{\beta}} T_{\beta \alpha}^{\gamma} .
\end{gathered}
$$

In other words, $E_{\alpha \beta}^{i j}$ is graded-antisymmetric both in lower indices and in upper indices and $T_{\alpha \beta}^{\gamma}$ is graded-antisymmetric in lower indices. The statistical parity of structure tensors is determined by the sum of the parities of the tensor indices, so that $\epsilon\left(R_{\alpha}^{i}\right)=$ $\left(\epsilon_{\alpha}+\epsilon_{i}\right)(\bmod 2), \epsilon\left(T_{\alpha \beta}^{\gamma}\right)=\left(\epsilon_{\alpha}+\epsilon_{\beta}+\epsilon_{\gamma}\right)(\bmod 2)$, and $\epsilon\left(E_{\alpha \beta}^{i j}\right)=\left(\epsilon_{i}+\epsilon_{j}+\epsilon_{\alpha}+\epsilon_{\beta}\right)$ $(\bmod 2)$.

The next step determines the restrictions imposed by the Jacobi identity. In general, it leads to new gauge-structure tensors and equations [172, 258, 84, 28]. The identity

$$
\sum_{\text {cyclic over } 1,2,3}\left[\delta_{1},\left[\delta_{2}, \delta_{3}\right]\right]=0,
$$

produces the following relations among the tensors $R, T$ and $E$

$$
\sum_{\text {cyclic over 1,2,3 }}\left(R_{\delta}^{i} A_{\alpha \beta \gamma}^{\delta}-S_{0, j} B_{\alpha \beta \gamma}^{j i}\right) \varepsilon_{1}^{\gamma} \varepsilon_{2}^{\beta} \varepsilon_{3}^{\alpha}=0
$$

where we have defined

$$
\begin{gathered}
3 A_{\alpha \beta \gamma}^{\delta} \equiv\left(T_{\alpha \beta, k}^{\delta} R_{\gamma}^{k}-T_{\alpha \eta}^{\delta} T_{\beta \gamma}^{\eta}\right)+ \\
(-1)^{\epsilon_{\alpha}\left(\epsilon_{\beta}+\epsilon_{\gamma}\right)}\left(T_{\beta \gamma, k}^{\delta} R_{\alpha}^{k}-T_{\beta \eta}^{\delta} T_{\gamma \alpha}^{\eta}\right)+(-1)^{\epsilon_{\gamma}\left(\epsilon_{\alpha}+\epsilon_{\beta}\right)}\left(T_{\gamma \alpha, k}^{\delta} R_{\beta}^{k}-T_{\gamma \eta}^{\delta} T_{\alpha \beta}^{\eta}\right)
\end{gathered}
$$

and

$$
\begin{aligned}
3 B_{\alpha \beta \gamma}^{j i} \equiv( & \left.E_{\alpha \beta, k}^{j i} R_{\gamma}^{k}-E_{\alpha \delta}^{j i} T_{\beta \gamma}^{\delta}-(-1)^{\epsilon_{i} \epsilon_{\alpha}} R_{\alpha, k}^{j} E_{\beta \gamma}^{k i}+(-1)^{\epsilon_{j}\left(\epsilon_{i}+\epsilon_{\alpha}\right)} R_{\alpha, k}^{i} E_{\beta \gamma}^{k j}\right) \\
& +(-1)^{\epsilon_{\alpha}\left(\epsilon_{\beta}+\epsilon_{\gamma}\right)}\left(\text { RHS of above line with } \begin{array}{c}
\alpha \rightarrow \beta \\
\beta \rightarrow \gamma \\
\gamma \rightarrow \alpha
\end{array}\right) \\
& +(-1)^{\epsilon_{\gamma}\left(\epsilon_{\alpha}+\epsilon_{\beta}\right)}\left(\text { RHS of first line with } \begin{array}{c}
\alpha \rightarrow \gamma \\
\beta \rightarrow \alpha \\
\gamma \rightarrow \beta
\end{array}\right)
\end{aligned}
$$


A useful but lengthy exercise is to derive Eq.(2.25).

If the theory is irreducible, the on-shell independence of the generators (2.14) and their completeness (2.12) leads to the following solution of Eq.(2.25)

$$
A_{\alpha \beta \gamma}^{\delta}=S_{0, j} D_{\alpha \beta \gamma}^{j \delta},
$$

where $D_{\alpha \beta \gamma}^{j \delta}$ are new structure functions.

On the other hand, using this solution in the original equation (2.25), one obtains the following condition on the $D_{\alpha \beta \gamma}^{j \delta}$

$$
\sum_{\text {cyclic over } \epsilon_{1}, \epsilon_{2}, \epsilon_{3}} S_{0, j}\left(B_{\alpha \beta \gamma}^{j i}-(-1)^{\epsilon_{j}\left(\epsilon_{i}+\epsilon_{\delta}\right)} R_{\delta}^{i} D_{\alpha \beta \gamma}^{j \delta}\right) \epsilon_{1}^{\gamma} \epsilon_{2}^{\beta} \epsilon_{3}^{\alpha}=0
$$

Again, the completeness of the generators implies that the general solution of the preceding equation is of the form

$$
B_{\alpha \beta \gamma}^{j i}+(-1)^{\epsilon_{i} \epsilon_{\delta}} R_{\delta}^{j} D_{\alpha \beta \gamma}^{i \delta}-(-1)^{\epsilon_{j}\left(\epsilon_{i}+\epsilon_{\delta}\right)} R_{\delta}^{i} D_{\alpha \beta \gamma}^{j \delta}=-S_{0, k} M_{\alpha \beta \gamma}^{k j i}
$$

where $M_{\alpha \beta \gamma}^{k j i}$ is graded antisymmetric in $i, j$, and $k$. In this way, the Jacobi identity leads to the existence of two new gauge-structure tensors $D_{\alpha \beta \gamma}^{j \delta}$ and $M_{\alpha \beta \gamma}^{k j i}$ which, for a generic theory, are different from zero and must satisfy Eqs.(2.28) and (2.30).

Continuing in the same way, that is to say, commuting more and more gauge transformations, new structure tensors with increasing numbers of indices are obtained. These tensors are the so-called structure functions of the gauge algebra and they determine the nature of the set of gauge transformations of the theory. The reader may not be aware of the higher-order tensors because in the simplest gauge theories, such as Yang-Mills, they vanish.

The tensors $A_{\alpha \beta \gamma}^{\delta}, B_{\alpha \beta \gamma}^{j i}, D_{\alpha \beta \gamma}^{j \delta}$ and $M_{\alpha \beta \gamma}^{k j i}$ are all graded-antisymmetric in $\alpha, \beta$ and $\gamma$. In addition $B_{\alpha \beta \gamma}^{j i}$ is graded-antisymmetric in $i$ and $j$ while $M_{\alpha \beta \gamma}^{k j i}$ is graded antisymmetric in $i, j$ and $k$. Here we summarize these properties

$$
\begin{gathered}
A_{\alpha \beta \gamma}^{\delta}=-(-1)^{\epsilon_{\alpha} \epsilon_{\beta}} A_{\beta \alpha \gamma}^{\delta}=-(-1)^{\epsilon_{\beta} \epsilon_{\gamma}} A_{\alpha \gamma \beta}^{\delta} \\
B_{\alpha \beta \gamma}^{j i}=-(-1)^{\epsilon_{\alpha} \epsilon_{\beta}} B_{\beta \alpha \gamma}^{j i}=-(-1)^{\epsilon_{\beta} \epsilon_{\gamma}} B_{\alpha \gamma \beta}^{j i}=-(-1)^{\epsilon_{i} \epsilon_{j}} B_{\alpha \beta \gamma}^{i j} \\
D_{\alpha \beta \gamma}^{j \delta}=-(-1)^{\epsilon_{\alpha} \epsilon_{\beta}} D_{\beta \alpha \gamma}^{j \delta}=-(-1)^{\epsilon_{\beta} \epsilon_{\gamma}} D_{\alpha \gamma \beta}^{j \delta}, \\
M_{\alpha \beta \gamma}^{k j i}=-(-1)^{\epsilon_{\alpha} \epsilon_{\beta}} M_{\beta \alpha \gamma}^{k j i}=-(-1)^{\epsilon_{\beta} \epsilon_{\gamma}} M_{\alpha \gamma \beta}^{k j i}=-(-1)^{\epsilon_{j} \epsilon_{k}} M_{\alpha \beta \gamma}^{j k i}=-(-1)^{\epsilon_{i} \epsilon_{j}} M_{\alpha \beta \gamma}^{k i j} .
\end{gathered}
$$

The statistical parity of any of the above tensors is given by the sum of the statistical parities of the indices of that tensor.

A useful device in the study of gauge-structure relations is to introduce ghost fields $\mathcal{C}^{\alpha}$ with opposite statistics to those of the gauge parameters $\varepsilon^{\alpha}$,

$$
\epsilon\left(\mathcal{C}^{\alpha}\right)=\epsilon_{\alpha}+1
$$


and to replace gauge parameters by ghosts, as is done in the BRST formalism [32]. The ghost fields obey the same boundary conditions as gauge parameters. The ghosts can be used as a compact way of writing the gauge-structure equations. However, in order to do this, the symmetry properties of $T_{\alpha \beta}^{\gamma}, E_{\alpha \beta}^{i j}, D_{\alpha \beta \gamma}^{j \delta}, M_{\alpha \beta \gamma}^{k j i}$, etc., need to be correctly incorporated. Note that these tensors are graded anti-symmetric in lowerindex gauge indices $\alpha, \beta$, etc., whereas the ghosts satisfy $\mathcal{C}^{\alpha} \mathcal{C}^{\beta}=(-1)^{\left(\epsilon_{\alpha}+1\right)\left(\epsilon_{\beta}+1\right)} \mathcal{C}^{\beta} \mathcal{C}^{\alpha}$. If one is given a graded anti-symmetric tensor $T_{\alpha_{1} \alpha_{2} \alpha_{3} \alpha_{4} \ldots}$, then a way to make it into a graded symmetric tensor with symmetry factors $\epsilon_{\alpha_{1}}+1, \epsilon_{\alpha_{2}}+1$, etc., associated with indices $\alpha_{1}, \alpha_{2}$, etc., is to multiply by a factor of $(-1)^{\epsilon_{\alpha_{i}}}$ for every other index $\alpha_{i}$ in $T_{\alpha_{1} \alpha_{2} \alpha_{3} \alpha_{4} \ldots}$. In other words, one replaces $T_{\alpha_{1} \alpha_{2} \alpha_{3} \alpha_{4} \ldots}$ by $(-1)^{\epsilon_{\alpha_{2}}+\epsilon_{\alpha_{4}}+\ldots} T_{\alpha_{1} \alpha_{2} \alpha_{3} \alpha_{4} \ldots}$. Using this device, one arrives at a compact way of writing the Noether identity (2.8), the gauge commutator relation (2.22), as well as Eqs.(2.28) and (2.30) which arise from the Jacobi identity:

$$
\begin{gathered}
S_{0, i} R_{\alpha}^{i} \mathcal{C}^{\alpha}=0 \\
\left(2 R_{\alpha, j}^{i} R_{\beta}^{j}-R_{\gamma}^{i} T_{\alpha \beta}^{\gamma}+S_{0, j} E_{\alpha \beta}^{j i}\right)(-1)^{\epsilon_{\alpha}} \mathcal{C}^{\beta} \mathcal{C}^{\alpha}=0 \\
\left(A_{\alpha \beta \gamma}^{\delta}-S_{0, j} D_{\alpha \beta \gamma}^{j \delta}\right)(-1)^{\epsilon_{\beta}} \mathcal{C}^{\gamma} \mathcal{C}^{\beta} \mathcal{C}^{\alpha}=0 \\
\left(B_{\alpha \beta \gamma}^{j i}+(-1)^{\epsilon_{i} \epsilon_{\delta}} R_{\delta}^{j} D_{\alpha \beta \gamma}^{i \delta}-(-1)^{\epsilon_{j}\left(\epsilon_{i}+\epsilon_{\delta}\right)} R_{\delta}^{i} D_{\alpha \beta \gamma}^{j \delta}+S_{0, k} M_{\alpha \beta \gamma}^{k j i}\right)(-1)^{\epsilon_{\beta}} \mathcal{C}^{\gamma} \mathcal{C}^{\beta} \mathcal{C}^{\alpha}=0
\end{gathered}
$$

where $A_{\alpha \beta \gamma}^{\delta}$ and $B_{\alpha \beta \gamma}^{j i}$ are defined in Eqs.(2.26) and (2.27). The graded-anticommuting nature of the ghosts automatically produces the appropriate graded-cyclic sums.

Equations (2.33) through (2.36) are key equations for an irreducible algebra.

Now let us consider a first-stage reducible gauge theory. In this case, the existence of non-trivial relations among the generators in Eq.(2.16) leads to the appearance of new tensor quantities.

For first-stage reducible theories there are on-shell null vectors for the generators $R_{\alpha}^{i}$. Let $Z_{a}^{\alpha}$ denote these null vectors. In Eq.(2.16), the $Z_{a}^{\alpha}$ are called $R_{1 a}^{\alpha}$ when $\alpha=\alpha_{0}$ and $a=\alpha_{1}$. The null vectors are independent on-shell. Their presence modifies the solutions of the Jacobi identities in Eqs. 2.35) and (2.36) as well as higher-commutator structure equations. In addition there are new structure equations. One of these is Eq.(2.16) itself:

$$
R_{\alpha}^{i} Z_{b}^{\alpha}=S_{0, j} V_{b}^{j i}
$$

Another is derived as follows. Take relation (2.22) and multiply it by $Z_{a}^{\beta}$ to obtain

$$
\left(R_{\alpha, j}^{i} R_{\beta}^{j}-(-1)^{\epsilon_{\alpha} \epsilon_{\beta}} R_{\beta, j}^{i} R_{\alpha}^{j}-R_{\gamma}^{i} T_{\alpha \beta}^{\gamma}+S_{0, j} E_{\alpha \beta}^{j i}\right) Z_{a}^{\beta}=0 .
$$

Use Eq.(2.37) to express $R_{\beta}^{j} Z_{a}^{\beta}$ as a term proportional to equations of motion. Also do the same with $R_{\beta, j}^{i} R_{\alpha}^{j} Z_{a}^{\beta}$ and make use of the Noether identity in Eq.(2.8). After 
a little algebra, one finds that the previous equation can be written in the form

$$
R_{\gamma}^{i}\left((-1)^{\epsilon_{a} \epsilon_{\beta}} Z_{a, j}^{\gamma} R_{\beta}^{j}-T_{\beta \delta}^{\gamma} Z_{a}^{\delta}\right)=S_{0, j} M_{\beta a}^{j i},
$$

for some quantity $M_{\beta a}^{j i}$. Terms proportional to the equations of motion have been collected into $M_{\beta a}^{j i}$. Using the completeness of the null vectors $Z_{a}^{\alpha}$, the general solution to this equation is

$$
(-1)^{\epsilon_{a} \epsilon_{\beta}} Z_{a, j}^{\gamma} R_{\beta}^{j}-T_{\beta \delta}^{\gamma} Z_{a}^{\delta}=-Z_{d}^{\gamma} A_{a \beta}^{d}-S_{0, j} G_{a \beta}^{j \gamma}
$$

Eq.(2.38) is a new gauge-structure equation for the first-stage reducible case. Two new structure tensors $A_{a \beta}^{d}$ and $G_{a \beta}^{j \gamma}$ arise.

The null vectors also lead to modifications of the solution of the Jacobi identity. Eq.(2.25) still holds but its solution is different. Instead of Eq.(2.35), one obtains

$$
\left(A_{\alpha \beta \gamma}^{\delta}+Z_{c}^{\delta} F_{\alpha \beta \gamma}^{c}-S_{0, j} D_{\alpha \beta \gamma}^{j \delta}\right)(-1)^{\epsilon_{\beta}} \mathcal{C}^{\gamma} \mathcal{C}^{\beta} \mathcal{C}^{\alpha}=0,
$$

where we have made use of the completeness of the null vectors $Z_{a}^{\alpha}$. In this equation $A_{\alpha \beta \gamma}^{\delta}$ stands for the combination of terms in Eq.(2.26).

Multiplying Eq.(2.39) by $R_{\delta}^{i}$ and using the Jacobi identity result of Eq.(2.25) lead to a modification of Eq.(2.29) involving $B_{\alpha \beta \gamma}^{i j}$. The new result reads

$$
S_{0, j}\left(B_{\alpha \beta \gamma}^{j i}-(-1)^{\epsilon_{j}\left(\epsilon_{i}+\epsilon_{\delta}\right)} R_{\delta}^{i} D_{\alpha \beta \gamma}^{j \delta}+V_{c}^{j i} F_{\alpha \beta \gamma}^{c}\right)(-1)^{\epsilon_{\beta}} \mathcal{C}^{\gamma} \mathcal{C}^{\beta} \mathcal{C}^{\alpha}=0
$$

when written using ghosts. The general solution is

$$
\begin{gathered}
\left(B_{\alpha \beta \gamma}^{j i}+(-1)^{\epsilon_{i} \epsilon_{\delta}} R_{\delta}^{j} D_{\alpha \beta \gamma}^{i \delta}-(-1)^{\epsilon_{j}\left(\epsilon_{i}+\epsilon_{\delta}\right)} R_{\delta}^{i} D_{\alpha \beta \gamma}^{j \delta}+\right. \\
\left.V_{c}^{j i} F_{\alpha \beta \gamma}^{c}+S_{0, k} M_{\alpha \beta \gamma}^{k j i}\right)(-1)^{\epsilon_{\beta}} \mathcal{C}^{\gamma} \mathcal{C}^{\beta} \mathcal{C}^{\alpha}=0
\end{gathered}
$$

where $B_{\alpha \beta \gamma}^{j i}$ is given in Eq.(2.27).

By taking more and more commutators of gauge transformations, more structure functions and equations appear, some of which involve graded symmetrizations in the first-stage gauge indices $a, b$, etc.. As in the irreducible case, it is useful to introduce ghosts $\eta^{a}$ to automatically incorporate graded symmetrization. Equations (2.37) and (2.38) can then be written as

$$
\left(R_{\beta}^{i} Z_{a}^{\beta}-S_{0, j} V_{a}^{j i}\right) \eta^{a}=0
$$

and

$$
\left((-1)^{\epsilon_{a} \epsilon_{\beta}} Z_{a, j}^{\gamma} R_{\beta}^{j}-T_{\beta \delta}^{\gamma} Z_{a}^{\delta}+Z_{d}^{\gamma} A_{a \beta}^{d}+S_{0, j} G_{a \beta}^{j \gamma}\right) \eta^{a} C^{\beta}=0
$$


To summarize, key equations for first-stage reducible theories are Eqs.(2.33), (2.34) and (2.39) - (2.42). Besides the null vectors $Z_{a}^{\beta}$, the new structure tensors are $V_{a}^{j i}$, $A_{a \beta}^{d}, G_{a \beta}^{j \gamma}, F_{\alpha \beta \gamma}^{a}$ as well as higher-level tensors.

Needless to say, for a higher-order reducible theory the number of quantities and equations increases considerably. The complexity of the formalism makes the study of the gauge structure at higher levels quite complicated. A more sensible approach is to have a generating functional whose expansion in terms of auxiliary fields produces the generic gauge-structure tensors. In addition, it is desirable to have a simple single equation which, when expanded in terms of auxiliary fields, generates the entire set of gauge-structure equations. The field-antifield method [24, 25, 26] provides such a formalism. The generating functional for structure tensors is a generalized action subject to certain boundary conditions and the classical master equation contains all the gauge-structure equations. Before presenting the abstract machinery, it is of pedagogical value to consider some examples of the formalism of this section. 


\section{Examples of Gauge Theories}

This section presents eight gauge theories, which will be used in Sect. 5 to illustrate the antibracket formalism. The theories are (1) the spinless relativistic particle, (2) Yang-Mills theories, (3) four-dimensional topological Yang-Mills theories, (4) the fourdimensional antisymmetric tensor field, (5) abelian $p$-form theories, (6) the open bosonic string field theory, (7) the massless relativistic spinning particle, and (8) the first-quantized bosonic string. Models (1), (2), (7) and (8) are closed and irreducible. Models (3) and (4) are first-stage reducible. Model (5) is $p$-stage reducible and model (6) is an infinitely reducible open system. For each theory, the classical action $S_{0}$ and its gauge symmetries are first presented. Then, the non-zero gauge-structure tensors are obtained. The determination of the structure tensors is the first computational step in the antibracket formalism. The results in this section are used in Sect. 5 to obtain proper solutions $S$.

In the first subsection on the spinless relativistic particle, we illustrate the compact notation of Sect. 2.1. Models (1) and (8) are respectively one and two-dimensional gravity theories. Model (8), the first-quantized bosonic string, is used to explain the construction of general-coordinate-invariant theories, i.e., gravities. In the subsection 3.7 on the massless relativistic spinning particle, we provide a mini-review of supersymmetry and supergravity. A brief introduction to string field theory is given in Sect. 3.6.

As exercises for the reader, we suggest the following three computations. Verify that the gauge transformations leave $S_{0}$ invariant. (ii) Given $S_{0}$ and its gauge symmetries, obtain the results presented for the gauge-structure tensors. (iii) Verify Eqs. 2.33)-(2.36) for the irreducible theories, and verify Eqs.(2.33), (2.34) and (2.39) - (2.42) for the first-stage reducible theories.

\subsection{The Spinless Relativistic Particle}

One of the simplest examples of a model with a gauge invariance is the free relativistic particle. It actually corresponds to a $0+1$ dimensional gravity theory with scalar fields. The supersymmetric generalization of the spinless relativistic particle is presented in Sect. 3.7.

Let us use this system to illustrate the formalism in Sect. 2. The degrees of freedom are a particle coordinate $x^{\mu}$ and an einbein $e$ both of which are functions of a single proper time variable $\tau$. The action is given by

$$
S_{0}\left[x^{\mu}, e\right]=\int \mathrm{d} \tau \frac{1}{2}\left(\frac{\dot{x}_{\mu} \dot{x}^{\mu}}{e}-m^{2} e\right), \quad \phi^{i}=\left(x^{\mu}, e\right)
$$


where a dot over a variable indicates a derivative with respect to proper time. The variations of the action with respect to the fields, Eq.(2.6), are

$$
S_{0, \mu}=-\frac{d}{d \tau}\left(\frac{\dot{x}_{\mu}}{e}\right), \quad S_{0, e}=\frac{1}{2}\left(-\frac{\dot{x}^{2}}{e^{2}}-m^{2}\right)
$$

where $S_{0, e}$ is the variation of the action with respect to field $e$; in other words, we also use $e$ as a field index for the einbein $e$. If the equation of motion $S_{0, e}=0$ is used to solve for $e$, and this solution is substituted into the action in Eq.(3.1), one finds that the action becomes the familiar one: $S_{0}=-\int \mathrm{d} \tau m \sqrt{-\dot{x}^{2}}$. Classically, this action and the one in Eq.(3.1) are equivalent.

The infinitesimal gauge transformations for this system can be written as

$$
\delta x^{\mu}=\frac{\dot{x}^{\mu}}{e} \varepsilon, \quad \delta e=\dot{\varepsilon}
$$

It is straightforward to verify that Eq.(3.3) is a symmetry of Eq.(3.1). The Noether identity in Eq.(2.8) reads

$$
\int \mathrm{d} \tau\left\{-\left(\frac{\dot{x}_{\mu}}{e}\right) \frac{d}{d \tau}\left(\frac{\dot{x}^{\mu}}{e}\right)-\frac{1}{2}\left(\frac{\dot{x}^{2}}{e^{2}}+m^{2}\right) \frac{d}{d \tau}\right\} \delta\left(\tau-\tau^{\prime}\right)=0
$$

which is verified using integration by parts.

The transformations laws in Eq.(3.3) in the form of Eq.(2.5) are

$$
R^{\mu} \varepsilon=\frac{\dot{x}^{\mu}}{e} \varepsilon, \quad R^{e} \varepsilon=\frac{d}{d \tau} \varepsilon
$$

Eq.(3.5) says that $R^{\mu}$ is the operator that is multiplication by $\frac{\dot{x}^{\mu}}{e}$ and $R^{e}$ is $\frac{d}{d \tau}$. In kernel form using compact index notation, they are

$$
R_{\sigma}^{\mu \tau}=\frac{\dot{x}^{\mu}(\tau)}{e} \delta(\tau-\sigma), \quad R_{\sigma}^{e \tau}=\frac{d}{d \tau} \delta(\tau-\sigma)
$$

Recall that in using compact notation the index $\alpha$ of $R_{\alpha}^{i}$ in Eq.(2.5) represents not only a discrete index labelling the different gauge transformations but also a spacetime index. Since there is only one type of gauge transformation the discrete index takes on only one value, which we drop for convenience. Hence the index $\alpha$ of $R_{\alpha}^{i}$ is replaced by the space-time variable $\sigma$. In this subsection, we use the Greek letters $\rho$, $\sigma, \tau$ and $v$ to denote proper time variables. Likewise the index $i$ on $R_{\alpha}^{i}$ in Eq.(2.5) represents not only a field index $\mu$ or $e$ but also a proper time variable $\tau$.

The algebra of the gauge transformations is simply

$$
\left[\delta_{1}, \delta_{2}\right]=\left[\delta\left(\varepsilon_{1}\right), \delta\left(\varepsilon_{2}\right)\right]=0
$$


where $\delta\left(\varepsilon_{1}\right)$ indicates a gauge transformation with parameter $\varepsilon_{1}$. These abelian gauge transformations (3.3) are related to the standard reparametrization transformations

$$
\delta_{R} x^{\mu}=\dot{x}^{\mu} \varepsilon, \quad \delta_{R} e=\frac{d}{d \tau}(e \varepsilon)
$$

through the following redefinition of the gauge parameter

$$
\varepsilon \longrightarrow \varepsilon e .
$$

The algebra of reparametrization transformations reads

$$
\left[\delta_{R}\left(\varepsilon_{1}\right), \delta_{R}\left(\varepsilon_{2}\right)\right]=\delta_{R}\left(\varepsilon_{12}\right),
$$

with the parameter $\varepsilon_{12}$ given by

$$
\varepsilon_{12}=\dot{\varepsilon}_{1} \varepsilon_{2}-\varepsilon_{1} \dot{\varepsilon}_{2}
$$

Eqs.(3.7) and (3.8) correspond to the usual diffeomorphism algebra. Since the commutator of two gauge transformations is a gauge transformation, the algebra is closed and $E_{\alpha \beta}^{j i}$ in Eq.(2.23) is zero. This example illustrates the effect of field-dependent redefinitions of the gauge parameters or, equivalently, of the gauge generators: An abelian algebra can be transformed into a non-abelian one. The converse of this also holds. One can transform any given non-abelian algebra into an abelian algebra using field-dependent redefinitions, a result known as the abelianization theorem [27]. The fact that this process can spoil the locality of the transformations is one of the reasons for using the non-abelian version.

It may appear unusual that a single family of gauge transformations produces non-abelian commutation relations. This is due to the local non-commutativity of reparametrization transformations that arises from the time derivatives in Eq.(3.6). Indeed, when $\varepsilon_{1}$ and $\varepsilon_{2}$ have non-overlapping support, i.e., $\varepsilon_{1}(\tau)=0$ where $\varepsilon_{2}(\tau) \neq 0$ and vice-versa, $\varepsilon_{12}=0$.

It is instructive to see how a non-zero structure constant $T_{\alpha \beta}^{\gamma}$ for the diffeomorphism algebra arises using compact notation. In what follows, gauge indices, $\alpha, \beta$, etc. are replaced by proper time variable $\rho, \sigma, \tau, v$. From Eq.(3.6) one sees that the transformation operators $R$ for reparametrizations are

$$
\begin{gathered}
R_{\sigma}^{\mu \tau}=\dot{x}^{\mu}(\tau) \delta(\tau-\sigma) \\
R_{\sigma}^{e \tau}=e(\tau) \frac{d}{d \tau} \delta(\tau-\sigma)+\dot{e}(\tau) \delta(\tau-\sigma)=\frac{d}{d \tau}[e(\tau) \delta(\tau-\sigma)] .
\end{gathered}
$$

For the $x^{\mu}$ degrees of freedom, a straightforward computation yields

$$
R_{\sigma, \nu v}^{\mu \tau}=\delta_{\nu}^{\mu} \delta(\tau-\sigma) \frac{d}{d \tau} \delta(\tau-v)
$$




$$
\begin{aligned}
& R_{\sigma, \nu v}^{\mu \tau} R_{\rho}^{\nu v}=\sum_{\nu} \int \mathrm{d} v \delta_{\nu}^{\mu} \delta(\tau-\sigma) \frac{d}{d \tau} \delta(\tau-v) \dot{x}^{\nu}(v) \delta(v-\rho) \\
& =\ddot{x}^{\mu}(\tau) \delta(\tau-\sigma) \delta(\tau-\rho)+\dot{x}^{\mu}(\tau) \delta(\tau-\sigma) \frac{d}{d \tau} \delta(\tau-\rho)
\end{aligned}
$$

Antisymmetrizing in $\rho$ and $\sigma$ and comparing with Eq. (2.22) one finds

$$
T_{\sigma \rho}^{\tau}=\delta(\tau-\sigma) \frac{d}{d \tau} \delta(\tau-\rho)-\delta(\tau-\rho) \frac{d}{d \tau} \delta(\tau-\sigma)
$$

which is in agreement with Eqs.(3.7) and (3.8). For $e$, straightforward computation produces

$$
\begin{gathered}
R_{\sigma, e v}^{e \tau}=\delta(\tau-v) \frac{d}{d \tau} \delta(\tau-\sigma)+\delta(\tau-\sigma) \frac{d}{d \tau} \delta(\tau-v)=\frac{d}{d \tau}[\delta(\tau-v) \delta(\tau-\sigma)] \\
R_{\sigma, e v}^{e \tau} R_{\rho}^{e v}=e(\tau)\left[\frac{d}{d \tau} \delta(\tau-\sigma)\right]\left[\frac{d}{d \tau} \delta(\tau-\rho)\right]+\dot{e}(\tau) \delta(\tau-\rho) \frac{d}{d \tau} \delta(\tau-\sigma)+ \\
2 \dot{e}(\tau) \delta(\tau-\sigma) \frac{d}{d \tau} \delta(\tau-\rho)+e(\tau) \delta(\tau-\sigma) \frac{d^{2}}{d \tau^{2}} \delta(\tau-\rho)+\ddot{e}(\tau) \delta(\tau-\sigma) \delta(\tau-\rho)
\end{gathered}
$$

Antisymmetrizing in $\rho$ and $\sigma$ and using Eq.(2.23), one finds $T$ is again given by Eq.(3.9).

Although compact notation is useful to represent the formalism of gauge theories in full generality, it is cumbersome for specific theories, especially for those in which more natural notation has already been established. In the examples that follow, we do not explicitly display equations in compact form but use more conventional notation.

\subsection{Yang-Mills Theories}

Yang-Mills theories [276] are perhaps the most familiar gauge theories. For each Lie algebra $\mathcal{G}$ there is different theory. The fundamental fields are gauge potentials $A_{\mu}^{a}$ where there is an index $a$ for each generator $T_{a}$ of $\mathcal{G}$. In a matrix representation, the generators are antihermitian matrices which are conventionally normalized so that $\operatorname{Tr}\left(T_{a} T_{b}\right)=-\frac{1}{2} \delta_{a b}$. The generators satisfy

$$
\left[T_{a}, T_{b}\right]=f_{a b}^{c} T_{c}
$$

where $f_{a b}{ }^{c}$ are the structure constants of $\mathcal{G}$. They are real and antisymmetric in lower indices $f_{a b}{ }^{c}=-f_{b a}{ }^{c}$ and they must satisfy the Jacobi identity

$$
f_{a b}{ }^{e} f_{e c}{ }^{d}+f_{c a}^{e} f_{e b}{ }^{d}+f_{b c}{ }^{e} f_{e a}{ }^{d}=0 .
$$


The Yang-Mills action is

$$
S_{0}\left[A_{\mu}^{a}\right]=-\frac{1}{4} \int \mathrm{d}^{d} x F_{\mu \nu}^{a}(x) F_{a}^{\mu \nu}(x)=\frac{1}{2} \int \mathrm{d}^{d} x \operatorname{Tr}\left[F_{\mu \nu}(x) F^{\mu \nu}(x)\right],
$$

where $d$ is the dimension of space-time, $F_{\mu \nu}(x) \equiv F_{\mu \nu}^{a}(x) T_{a}$, and where the field strengths $F_{\mu \nu}^{a}$ are

$$
F_{\mu \nu}^{a}(x) \equiv \partial_{\mu} A_{\nu}^{a}(x)-\partial_{\nu} A_{\mu}^{a}(x)-f_{b c}{ }^{a} A_{\mu}^{b}(x) A_{\nu}^{c}(x)
$$

The equations of motion, gauge transformations and gauge algebra are

$$
\begin{gathered}
\left(D^{\mu} F_{\mu \nu}\right)_{a} \equiv D_{a}^{\mu}{ }_{a}^{b} F_{b \mu \nu}=0 \\
\delta A_{\mu}^{a}=\left(D_{\mu} \Lambda\right)^{a} \equiv D_{\mu b}^{a} \Lambda^{b}, \\
{\left[\delta\left(\Lambda_{1}\right), \delta\left(\Lambda_{2}\right)\right] A_{\mu}^{c}=\delta\left(\Lambda_{12}\right) A_{\mu}^{c}=D_{\mu d}^{c}\left(f_{a b}{ }^{d} \Lambda_{1}^{b} \Lambda_{2}^{a}\right),}
\end{gathered}
$$

so that $\Lambda_{12}^{c}=f_{a b}^{c} \Lambda_{1}^{b} \Lambda_{2}^{a}$. The covariant derivatives $D_{\mu b}^{a}$ and $D_{\mu a}{ }^{b}$ in the adjoint representation are

$$
\begin{gathered}
D_{\mu b}^{a}=\delta^{a}{ }_{b} \partial_{\mu}-f_{c b}{ }^{a} A_{\mu}^{c}, \\
D_{\mu a}{ }^{b}=\delta_{a}{ }^{b} \partial_{\mu}+f_{c a}{ }^{b} A_{\mu}^{c},
\end{gathered},
$$

where $D_{\mu b}^{a}$ is applied to fields $\phi^{b}$ with an upper index $b$ and $D_{\mu a}{ }^{b}$ is applied to fields $\phi_{b}$ with a lower index $b$. One has

$$
\int \mathrm{d}^{d} x \phi_{a} D_{\mu b}^{a} \phi^{b}=-\int \mathrm{d}^{d} x\left(D_{\mu b}^{a} \phi_{a}\right) \phi^{b}
$$

The operator $R_{\alpha}^{i}$ in Eq.(2.5) corresponds to $D^{\mu a}{ }_{b}$. The covariant derivative satisfies

$$
\begin{aligned}
& {\left[\begin{array}{ll}
D_{\mu}, D_{\nu} & { }^{a}{ }_{b}=-f_{c b}{ }^{a} F_{\mu \nu}^{c},
\end{array}\right.} \\
& {\left[D_{\mu}, D_{\nu}\right]_{a}^{b}=f_{c a}{ }^{b} F_{\mu \nu}^{c} .}
\end{aligned}
$$

In using compact notation, the spatial dependence as well as index dependence of tensors needs to be specified. For local theories, the spatial dependence is proportional to delta functions or a finite number of derivatives acting on delta functions. When the spatial-temporal part of a tensor structure is a delta function, it is proportional to the identity operator in $x$-space when regarded as an operator. In such cases, it is convenient to drop explicitly such identity operators.

Eq.(3.16) is in the form of Eq.(2.23) with $E_{\alpha \beta}^{j i}=0$ and $T_{a b}^{c}=f_{a b}{ }^{c}$ where two identity operators or delta functions are implicit. One concludes that this example constitutes a closed, irreducible gauge algebra. 
It is useful to verify the key equations for an irreducible closed algebra given in Eqs.(2.33)-(2.36). The generator of gauge transformations is the covariant derivative in Eq.(3.17). Using Eqs.(3.14) and (3.15), the Noether identity in Eq.(2.33) reads

$$
\int \mathrm{d}^{d} x\left(D^{\mu} F_{\mu \nu}\right)_{b}\left(D^{\nu} \mathcal{C}\right)^{b}=0
$$

To verify this equation, integrate by parts, use the antisymmetry of $F_{\mu \nu}$ in $\mu$ and $\nu$, use Eq.(3.19), and then make use of the antisymmetry of $f_{c d}{ }^{a}$ in $c$ and $d$ :

$$
\begin{aligned}
\int \mathrm{d}^{d} x\left(D^{\mu} F_{\mu \nu}\right)_{b}\left(D^{\nu} \mathcal{C}\right)^{b} & =-\int \mathrm{d}^{d} x\left(D^{\nu} D^{\mu} F_{\mu \nu}\right)_{b} \mathcal{C}^{b}= \\
-\frac{1}{2} \int \mathrm{d}^{d} x\left(\left[D^{\nu}, D^{\mu}\right] F_{\mu \nu}\right)_{b} \mathcal{C}^{b} & =-\frac{1}{2} \int \mathrm{d}^{d} x f_{b d}^{a} F_{a}^{\nu \mu} F_{\mu \nu}^{d} \mathcal{C}^{b}=0 .
\end{aligned}
$$

A straightforward computation of the $2 R_{\alpha, j}^{i} R_{\beta}^{j} \mathcal{C}^{\beta} \mathcal{C}^{\alpha}$ term in the commutator algebra equation of Eq. (2.34) produces $-2 f_{b a}{ }^{c}\left(\partial^{\mu} \mathcal{C}^{b}-A^{d \mu} f_{d e}{ }^{b} \mathcal{C}^{e}\right) \mathcal{C}^{a}$ which, after a little algebra that makes use of Eq.(3.11), leads to $D^{\mu c}{ }_{d}\left(f_{a b}{ }^{d} \mathcal{C}^{b} \mathcal{C}^{a}\right)$. Using this for $2 R_{\alpha, j}^{i} R_{\beta}^{j} \mathcal{C}^{\beta} \mathcal{C}^{\alpha}$ in Eq.(2.34), one concludes, as expected, that $T_{a b}^{c}=f_{a b}{ }^{c}$ and $E_{\alpha \beta}^{j i}=0$. When $T_{a b}^{c}=f_{a b}{ }^{c}$ is used, the gauge-structure Jacobi equation in Eq. (2.35) leads to $3 A_{a b c}^{d}=-($ LHS of Eq.(3.11) $)$ so that $A_{a b c}^{d}=0$. Finally, the other consequence of the Jacobi identity, namely Eq.(2.36), produces the tautology $0=0$. All terms are zero because the tensors $B_{\alpha \beta \gamma}^{j i}, D_{\alpha \beta \gamma}^{i \delta}$ and $M_{\alpha \beta \gamma}^{k j i}$ are all zero. Higher-level equations (which were not displayed in Sect. 2.4) are automatically satisfied because higher-level tensors are identically zero.

\subsection{Topological Yang-Mills Theory}

In four-dimensions, the action for topological Yang-Mills theory [272, 35] is proportional to the Pontrjagin index

$$
S_{0}=\frac{1}{4} \int \mathrm{d}^{4} x F_{\mu \nu}^{a *} F_{a}^{\mu \nu}
$$

where the dual field strength ${ }^{*} F_{\mu \nu}^{a}$ is given by ${ }^{*} F_{\mu \nu}^{a}=\frac{1}{2} \epsilon_{\mu \nu \rho \sigma} F^{a \rho \sigma}$, and where $\epsilon_{0123}=1$. The interest in this system is its connection [272] to Donaldson theory [90].

This action is invariant under the gauge transformations in Eq.(3.15) because the lagrangian is constructed as a group invariant of the field strength. In addition, since the theory is topological, the transformation

$$
\delta A_{\mu}^{a}=\varepsilon_{\mu}^{a},
$$


leaves the action invariant, as a short calculation verifies. The two gauge transformations form a closed algebra since

$$
\begin{gathered}
{\left[\delta\left(\Lambda_{1}, \varepsilon_{1}\right), \delta\left(\Lambda_{2}, \varepsilon_{2}\right)\right] A_{\mu}=\delta\left(\Lambda_{12}, \varepsilon_{12}\right) A_{\mu},} \\
\Lambda_{12}^{c} \equiv f_{a b}^{c} \Lambda_{1}^{b} \Lambda_{2}^{a}, \quad \varepsilon_{\mu 12}^{c} \equiv f_{a b}^{c}\left(\varepsilon_{\mu 1}^{b} \Lambda_{2}^{a}+\Lambda_{1}^{b} \varepsilon_{\mu 2}^{a}\right)
\end{gathered}
$$

However, the gauge generators are obviously off-shell linearly dependent since the $\varepsilon_{\mu}$ transformations include ordinary gauge transformations when $\varepsilon_{\mu}=D_{\mu} \Lambda$. Since the gauge transformations are not all independent, one has a reducible gauge theory and the coefficients $Z_{a}^{\alpha}$ in Eq. 2.37) and $A_{a \alpha}^{b}$ introduced in Eq.(2.38) are non-zero. Of course, the theory can be made irreducible by eliminating ordinary gauge transformations from the set of all transformations. However, for other theories it is not so easy to reduce the full set to an irreducible subset without spoiling locality or relativistic covariance and often it is convenient to formulate the theory as a reducible system.

Let us use topological Yang-Mills theory to illustrate the gauge-structure formalism of Sect. 2.4. The field index $i$ corresponds to both a gauge index and a vector Lorentz index since the field is $A^{a \mu}: i \leftrightarrow a \mu$. There are two types of gauge transformations so that the gauge index $\alpha$ of Sect. 2.4 corresponds to the group index $b$ in the case of an ordinary gauge transformation or to the pair $c \mu$ in the case of a topological gauge transformation: $\alpha \leftrightarrow(b, c \mu)$. The generator of ordinary gauge transformations is the covariant derivative in Eq.(3.17) and the generator of topological transformations is a delta function:

$$
R_{b}^{a \mu}=D_{b}^{\mu a}, \quad R_{b \nu}^{a \mu}=\delta_{b}^{a} \delta_{\nu}^{\mu}
$$

The null vectors, denoted as $Z_{b}^{\alpha}$ in Sect. 2.4, are

$$
Z_{b}^{a}=\delta_{b}^{a}, \quad Z_{b}^{a \mu}=-D_{b}^{\mu a}
$$

The number of null vectors is equal to the number of gauge generators. It is easily verified that Eq.(2.41) holds since $R_{c}^{b \mu} Z_{a}^{c} \eta^{a}+R_{c \nu}^{b \mu} Z_{a}^{c \nu} \eta^{a}=D^{\mu b}{ }_{c} \delta_{a}^{c} \eta^{a}+\delta_{c}^{b} \delta_{\nu}^{\mu}\left(-D^{\nu c}{ }_{a} \eta^{a}\right)=$ 0 . This computation implies that $V_{a}^{j i}=0$ in Eq.(2.41).

The non-zero structure constants are

$$
T_{a b}^{c}=f_{a b}^{c}, \quad T_{a \mu b}^{c \nu}=T_{a b \mu}^{c \nu}=\delta_{\mu}^{\nu} f_{a b}^{c},
$$

whereas $T_{a \mu b}^{c}=T_{a b \mu}^{c}=T_{a b}^{c \nu}=T_{a \mu b \nu}^{c \lambda}=T_{a \mu b \nu}^{c \lambda}=0$. As in the example of Sect. 3.2, $A_{\alpha \beta \gamma}^{\delta}$ is zero since $3 A_{a b c}^{d}=-\left(f_{a e}{ }^{d} f_{b c}{ }^{e}+f_{b e}{ }^{d} f_{c a}{ }^{e}+f_{c e}{ }^{d} f_{a b}{ }^{e}\right)=0$. One also finds $A_{a \mu b c}^{d \nu}=A_{a b \mu c}^{d \nu}=A_{a b c \mu}^{d \nu}=\delta_{\mu}^{\nu} A_{a b c}^{d}=0$. 
Eq. 2.39) holds because $A_{\alpha \beta \gamma}^{\delta}=F_{\alpha \beta \gamma}^{c}=D_{\alpha \beta \gamma}^{j \delta}=0$. Eq. 2.40) holds because $B_{\alpha \beta \gamma}^{j i}$ and $M_{\alpha \beta \gamma}^{k j i}$ are also zero. Finally, Eq.(2.42) is valid as long as $G_{a \beta}^{j \gamma}=0$ and

$$
A_{a b}^{c}=-f_{a b}^{c}, \quad A_{a b \mu}^{c}=0
$$

In verifying Eq.(2.42) it is useful to note that $Z_{a, b \mu}^{c \nu}=-\delta_{\mu}^{\nu} f_{a b}^{c}$, and $Z_{a, j}^{c}=0$ both when $j=b$ and when $j=b \mu$. For Eq.(2.42), there are four cases to verify: (i) $\gamma=c$ and $\beta=b$, (ii) $\gamma=c$ and $\beta=b \mu$, (iii) $\gamma=c \nu$ and $\beta=b$ and (iv) $\gamma=c \nu$ and $\beta=b \mu$. Case (i) gives $-f_{b d}^{c} \delta_{a}^{d}-\delta_{d}^{c} A_{a b \mu}^{d}=0$. Case (ii) is automatically zero because each term in Eq.(2.42) is zero. Case (iii), after a little algebra, results in the expression $f_{b a}{ }^{c} \partial^{\nu}\left(\eta^{a} \mathcal{C}^{b}\right)+f_{a b}{ }^{c} \partial^{\nu}\left(\eta^{a} \mathcal{C}^{b}\right)+\left(f_{a d}^{c} f_{b e}{ }^{d}+f_{b d}{ }^{c} f_{e a}{ }^{d}+f_{e d}{ }^{c} f_{a b}{ }^{d}\right) \eta^{a} \mathcal{C}^{b} A^{e \nu}$ which is zero because of the antisymmetry of $f_{a b}{ }^{c}$ in $a b$ and the Jacobi identity for the Lie algebra structure constants in Eq.(3.11). For case (iv), one finds $\delta_{\mu}^{\nu} f_{a b}{ }^{c}-\delta_{\mu}^{\nu} f_{b a}{ }^{c}=0$. In short, the important non-zero gauge structure tensors are given in Eqs.(3.23) - (3.26). Eqs.(2.33), (2.34) and (2.39) - (2.42) are all satisfied.

In a topological theory, the number of local degrees of freedom is zero. One finds that $n_{\text {dof }}$ is $4 N$ (for $A^{a \mu}$ ) minus $4 N$ (for $\varepsilon_{\mu}^{a}$ ) minus $N$ (for $\Lambda^{a}$ ) plus $N$ (for the null vectors in Eq.(3.24)). Hence, the net number of local degrees of freedom is zero.

\subsection{The Antisymmetric Tensor Field Theory}

Another example of first-stage reducible theory is the antisymmetric tensor gauge theory. Consider a tensor field $B_{\mu \nu}^{a}$ in four dimensions satisfying $B_{\nu \mu}^{a}=-B_{\mu \nu}^{a}$ whose dynamics is described by the action [112, 242]

$$
S_{0}\left[A_{\mu}^{a}, B_{\mu \nu}^{a}\right]=\int \mathrm{d}^{4} x\left(\frac{1}{2} A_{\mu}^{a} A_{a}^{\mu}-\frac{1}{2} B_{\mu \nu}^{a} F_{a}^{\mu \nu}\right)
$$

where $A_{\mu}^{a}$ is an auxiliary vector field. The field strength $F_{\mu \nu}^{a}$ is given in terms of $A_{\mu}^{a}$ as in the Yang-Mills case via Eq.(3.13). The action is invariant under the gauge transformations

$$
\delta B_{\kappa \lambda}^{a}=\epsilon_{\kappa \lambda \mu \nu} D^{\mu a}{ }_{b} \Lambda^{b \nu}, \quad \delta A^{a \mu}=0 .
$$

The covariant derivative $D^{\mu a}{ }_{b}$ is given in Eq.(3.17). The equations of motion derived from Eq. 3.27) are

$$
\frac{\partial_{r} S_{0}}{\partial B_{\mu \nu}^{a}}=-F_{a}^{\mu \nu}=0, \quad \frac{\partial_{r} S_{0}}{\partial A_{a}^{\mu}}=A_{\mu}^{a}+D_{b}^{\nu a} B_{\nu \mu}^{b}=0
$$

In spite of the presence of Lie-algebra structure constants $f_{a b}{ }^{c}$, the model has an abelian gauge algebra, i.e., $T_{\alpha \beta}^{\gamma}=0$, due to the fact that the vector field, which appears in the covariant derivative, does not transform. 
The gauge transformations (3.28) have an on-shell null vector. Indeed, taking

$$
\Lambda^{b \nu}=D^{\nu b} \xi^{c}
$$

one finds using Eq.(3.19) that

$$
\delta B_{\kappa \lambda}^{a}=\epsilon_{\kappa \lambda \mu \nu}\left(D^{\mu} D^{\nu}\right)^{a}{ }_{b} \xi^{b}=-\frac{1}{2} \epsilon_{\kappa \lambda \mu \nu} f_{c b}{ }^{a} F^{c \mu \nu} \xi^{b},
$$

which vanishes on-shell, since $F^{c \mu \nu}=0$ when the equations of motion (3.29) are used. Since the null vectors are independent, this theory is on-shell first-stage reducible.

It is instructive to determine the gauge-structure tensors and verify Eqs.(2.33), (2.34) and (2.39) - (2.42). The field index $i$ in Sect. 2 corresponds to $a \mu \nu$ in the case of the antisymmetric field $B^{a \mu \nu}$ and corresponds to $a \mu$ in the case of $A^{a \mu}$. The gauge index $\alpha$ of Sect. 2 corresponds to $b \nu$ which are the indices of $\Lambda^{b \nu}$. The null index $a$ of Sect. 2 is a Lie algebra generator index. The generator of gauge transformations $R_{\beta}^{i}$ is

$$
R_{b \lambda}^{a \mu \nu}=\epsilon^{\mu \nu \rho \sigma} \eta_{\sigma \lambda} D_{\rho b}^{a}, \quad R_{b \lambda}^{a \mu}=0,
$$

where the second equation holds because $A^{a \mu}$ does not transform and $\eta_{\sigma \lambda}$ is the flat space-time metric. The null vectors $Z_{a}^{\alpha}$ are the covariant derivative operators

$$
Z_{a}^{b \lambda}=D_{a}^{\lambda b}
$$

Using Eq.(2.41), one finds $R_{b \lambda}^{c \mu \nu} Z_{a}^{b \lambda}=\frac{1}{2} \epsilon^{\mu \nu \rho \sigma} f_{a b}{ }^{c} F_{\rho \sigma}^{b}$ so that

$$
\begin{gathered}
V_{a}^{b \rho \sigma c \mu \nu}=-\epsilon^{\mu \nu \rho \sigma} \delta^{b d} f_{a d}^{c}, \\
V_{a}^{j i}=0, \quad \text { if } j=b \nu \text { or } i=b \nu,
\end{gathered}
$$

that is, $V_{a}^{j i}=0$ if $i$ or $j$ corresponds to the field index of $A^{b \nu}$. The derived quantities $A_{\alpha \beta \gamma}^{\delta}$ and $B_{\alpha \beta \gamma}^{i j}$ are zero. Other gauge-structure tensors also vanish:

$$
T_{\alpha \beta}^{\gamma}=E_{\alpha \beta}^{j i}=D_{\alpha \beta \gamma}^{j \delta}=F_{\alpha \beta \gamma}^{c}=M_{\alpha \beta \gamma}^{k j i}=A_{a \beta}^{d}=0 .
$$

The gauge-structure equations are all satisfied. Eq.(2.33) holds because the action is invariant under gauge transformations, as is easily checked. Eq.(2.41) is satisfied. In fact, it was used above in Eq.(3.33) to obtain $V_{a}^{j i}$. Eqs.(2.39) and (2.40) are satisfied because all the tensors entering these equations are zero. Each term in Eq.(2.34) is zero: The first term $R_{\alpha, j}^{i} R_{\beta}^{j}$ is zero because $R_{\alpha, j}^{i}=0$ when $j=a \mu \nu$, i.e., when $j$ is a field index of $B^{a \mu \nu}$, and $R_{\beta}^{j}=0$ when $j=a \mu$, i.e., when $j$ is a field index of $A^{a \mu}$. The other terms vanish because the structure tensors vanish. Likewise each term in Eq.(2.42) is zero: The first term $Z_{\alpha, j}^{\gamma} R_{\beta}^{j}$ is zero because $Z_{\alpha, j}^{\gamma}$ like $R_{\alpha, j}^{i}$ is zero when $j=a \mu \nu$. 
Let $N$ be the number of generators of $\mathcal{G}$, i.e., the dimension of the Lie algebra $\mathcal{G}$. The number of degrees of freedom $n_{\text {dof }}$ is $4 N$ for $A^{a \mu}$ plus $6 N$ for $B_{\mu \nu}^{a}$ minus the number of gauge transformations $4 N$ plus the number of null vectors $N$, so that Eq.(2.20) reads $n_{\text {dof }}=7 N$.

\subsection{Abelian $p$-Form Theories}

It is not hard to find an example of an $L$-th stage reducible theory. Let $A$ be a $p$-form and define $F$ to be its field strength: $F=d A$ where $d$ is the exterior derivative. For $p+1$ less than the dimension $d$ of spacetime, an action for this theory is

$$
S_{0}=-\frac{1}{2} \int F \wedge^{*} F
$$

where $*$ is the dual star operation that takes a $q$-form into a $d-q$ form and $\wedge$ is the wedge product. On basis $q$-forms, it is defined by

$$
\begin{gathered}
*\left(d x^{\mu_{1}} \wedge d x^{\mu_{2}} \wedge \ldots \wedge d x^{\mu_{q}}\right)= \\
\frac{1}{(n-p) !} \varepsilon^{\mu_{1} \mu_{2} \ldots \mu_{d}} \eta_{\mu_{q+1} \nu_{q+1}} \eta_{\mu_{q+2} \nu_{q+2}} \ldots \eta_{\mu_{d} \nu_{d}} d x^{\nu_{q+1}} \wedge d x^{\nu_{q+2}} \wedge \ldots \wedge d x^{\nu_{d}},
\end{gathered}
$$

where $\eta_{\mu \nu}$ is the flat space-time metric and $\varepsilon^{\mu_{1} \mu_{2} \ldots \mu_{d}}$ is the antisymmetric tensor symbol. The case $p=1$ corresponds to abelian Yang-Mills theory. Using $d d=0$, one sees that the action is invariant under the gauge transformation

$$
\delta A=d \lambda_{p-1}
$$

where $\lambda_{p-1}$ is a $p-1$ form. This gauge transformation has its own gauge invariance. In fact, there is a tower of gauge invariances for gauge invariances given by

$$
\begin{aligned}
\delta \lambda_{p-1} & =d \lambda_{p-2}, \\
& \vdots \\
\delta \lambda_{1} & =d \lambda_{0},
\end{aligned}
$$

where $\lambda_{q}$ is a $q$-form. Hence, the theory is $p-1$ stage reducible. The number of degrees of freedom is

$$
n_{\mathrm{dof}}=\left(\begin{array}{l}
d \\
p
\end{array}\right)-\left(\begin{array}{c}
d \\
p-1
\end{array}\right)+\left(\begin{array}{c}
d \\
p-2
\end{array}\right)-\ldots-(-1)^{p}\left(\begin{array}{l}
d \\
1
\end{array}\right)+(-1)^{p}\left(\begin{array}{l}
d \\
0
\end{array}\right)=\left(\begin{array}{c}
d-1 \\
p
\end{array}\right)
$$

Note that $\left(\begin{array}{l}d \\ q\end{array}\right)$ is the dimension of the space of $q$-forms in $d$ dimensional space-time. 
The gauge generators at the $s$-th stage, $R_{s \alpha_{s}}^{\alpha_{s}-1}$, correspond to the exterior derivative $d$ acting on the space of $(p-1-s)$ forms, $d^{(p-1-s)}$ :

$$
\begin{aligned}
R_{0} & \leftrightarrow d^{(p-1)} \\
R_{1} & \leftrightarrow d^{(p-2)} \\
& \vdots \\
R_{p-1} & \leftrightarrow d^{(0)} .
\end{aligned}
$$

Because $d d=0$, Eq.(2.19) holds off-shell and all $V_{s \alpha_{s}}^{i \alpha_{s-2}}$ are zero. With the exception of the gauge generators, all gauge-structure tensors are zero.

If $n(p+1)=d=$ dimension of space time, a topological term can be added to the action

$$
\Delta S_{0}=\int \underbrace{F \wedge F \wedge \wedge F}_{n \text { terms }} .
$$

For $n=2$ one can consider the quantization of $\Delta S_{0}$ alone if $d=2(p+1)$. This would be another example of a topological theory.

Abelian $p$-form theories provide a good background for covariant open string field theory, a non-abelian generalization of $p$-form theory which is infinite-stage reducible.

\subsection{Open Bosonic String Field Theory}

The covariant $d=26$ open string field theory was obtained by E. Witten [270]. It resembles a Chern-Simons theory. The fundamental object is a string field $A$. Although one can proceed without a detailed understanding of $A, A$ can be expanded as a series in first-quantized string states whose coefficients are ordinary particle fields. Each member of this infinite tower of states corresponds to a particular vibrational mode of the string. In this manner, string theory is able to incorporate collectively many particles. For example, the open bosonic string possesses a tachyonic scalar, a massless vector field, and numerous massive states of all possible spins. For reviews on open bosonic string field theory, see refs. 215, 168, 247. Also useful is the discussion in Sect. 7.7, where the first quantization of the bosonic string is treated.

Covariant open string field theory can be formulated axiomatically [270, 271]. Fields are classified according to their string ghost number. If the string ghost number of $B$ is $g(B)=p$ then we say that $B$ is a string $p$-form in a generalized sense. The ingredients of abstract string theory are a derivation $Q$, a star operation $*$ which combines pairs of fields to produce a new field, and an integration operation $\int$ which yields a complex number $\int B$ for each integration over a string field $B$. These objects satisfy five axioms: 
(1) The nilpotency of $Q: Q Q=0$.

(2) Absence of surface terms in integration: $\int Q A=0$. This axiom is equivalent to an integration-by-parts rule.

(3) Graded distributive property of $Q$ across *:

$Q(A * B)=Q A * B+(-1)^{g(A)} A * Q B$.

(4) Associativity of the star product: $(A * B) * C=A *(B * C)$.

(5) Graded commutativity of the star product under the integral: $\int A * B=(-1)^{g(A) g(B)} \int B * A$.

The ghost number of a star product of fields is the sum of their ghost numbers: $g(A * B)=g(A)+g(B)$. The derivation $Q$ increases the ghost number by $1: g(Q A)=$ $g(A)+1$. In some circles, including refs. [270, 271], the ghost number is shifted by $-3 / 2$ so that $A$ has ghost number $-1 / 2$ instead of 1 .

The axioms are satisfied for non-abelian Chern-Simons theory in three dimensions. The field is a non-abelian vector potential which is converted into a Lie-algebravalued 1-form by multiplying by $d x^{\mu}: A^{a}{ }_{b} \equiv A_{\mu b}^{a} d x^{\mu} \equiv A_{\mu}^{s}\left(T_{s}\right)^{a}{ }_{b} d x^{\mu}$, where the $T_{s}$ are a set of matrix generators for the Lie group. The derivation $Q$ is the exterior derivative $d$. The star product is the wedge product and a matrix multiplication: $(A * B)_{b}^{a}=A^{a}{ }_{c} \wedge B^{c}{ }_{b}$. Integration is an integral over a three-dimensional manifold $M$ without boundary and a trace over the Lie-algebra indices: $\int A \equiv \int_{M} A_{a}^{a}$. Axiom (1) is satisfied because $d d=0$. Axiom (2) holds because $M$ has no boundary. Axiom (3) is satisfied because the exterior derivative $d$ is graded distributive across the wedge product. Axiom (4) holds because both the wedge product and matrix multiplication are associative. Finally, axiom (5) is satisfied because of the graded antisymmetry of the wedge product and the cyclic property of a trace: $\operatorname{Tr}(M N)=\operatorname{Tr}(N M)$ for any two matrices $M$ and $N$. In this example and in open string field theory, $\int B=0$ unless $B$ is a string 3 -form.

The action for the string 1 -form $A$ is given by

$$
S_{0}[A]=\frac{1}{2} \int A * Q A+\frac{1}{3} \int A * A * A .
$$

Using the above five axioms it is straightforward to show that the action is invariant under the gauge transformation

$$
\delta A=Q \Lambda_{0}+A * \Lambda_{0}-\Lambda_{0} * A,
$$


where $\Lambda_{0}$ is any string 0 -form. The equation of motion for $A$ reads

$$
F \equiv Q A+A * A=0 \quad .
$$

For the open bosonic string, $Q$ is the BRST charge $Q_{B R S T}$ of the first-quantized theory promoted to an operator by second quantization (see Eq.(7.51) with $\rho=$ $1)$. The $*$ product is intuitively described as follows. Let $\sigma$ be the parameter that determines a point on the string, so that $\sigma=0$ corresponds to one endpoint and $\sigma=\pi$ corresponds to the other endpoint. Divide the string in two at $\sigma=\pi / 2$ and call the two halves the left and right halves. Let $C=A * B$. Then * "glues" the left half of $A$ to the right half of $B$ by delta functions and what remains is $C$ so that the left half of $C$ is the right half of $A$ and the right half of $C$ is the left half of $B$. See Fig. 1. The star operator can be thought of as matrix multiplication if the range $0 \leq \sigma \leq \pi / 2$ of points of the string is associated with one matrix index and the range $\pi / 2 \leq \sigma \leq \pi$ is associated with the other matrix index. The integral operator is a delta function equating the left and right halves. See Fig. 2. In the matrix analogy, it is the trace. Precise definitions of $Q, *$ and $\int$ in terms of the vibrational modes of the string, i.e., particle excitations, can be found in refs. [68, 214, 142, 143].

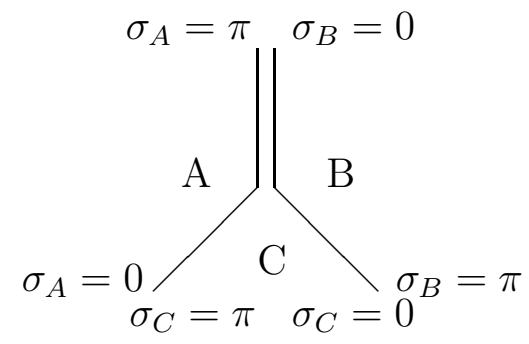

Figure 1. The String Star Product

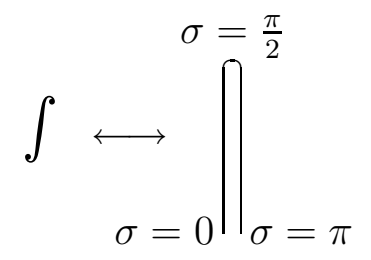

Figure 2. The String Integral

Unlike the three-dimensional Chern-Simons theory, the open bosonic string theory possesses $q$-forms for $q<0$. As a consequence there are on-shell gauge invariances for gauge invariances at all levels:

$$
\delta \Lambda_{0}=Q \Lambda_{-1}+A * \Lambda_{-1}+\Lambda_{-1} * A,
$$




$$
\begin{gathered}
\delta \Lambda_{-1}=Q \Lambda_{-2}+A * \Lambda_{-2}-\Lambda_{-2} * A \\
\vdots \\
\delta \Lambda_{-n}=Q \Lambda_{-n-1}+A * \Lambda_{-n-1}-(-1)^{n+1} \Lambda_{-n-1} * A,
\end{gathered}
$$

where $\Lambda_{q}$ is a string $q$-form. To verify these gauge invariances, note that if $\Lambda_{-n+1}=$ $Q \Lambda_{-n}+A * \Lambda_{-n}-(-1)^{n} \Lambda_{-n} * A$ and if one changes $\Lambda_{-n}$ by $\delta \Lambda_{-n}=Q \Lambda_{-n-1}+A *$ $\Lambda_{-n-1}-(-1)^{n+1} \Lambda_{-n-1} * A$ then $\Lambda_{-n+1}$ changes by $\delta \Lambda_{-n+1}=(Q A+A * A) * \Lambda_{-n-1}-$ $\Lambda_{-n-1} *(Q A+A * A)$, as a little algebra reveals. As a consequence, this change $\delta \Lambda_{-n+1}$ in $\Lambda_{-n+1}$ is zero when the equations of motion in Eq.(3.41) are invoked. One of the confusing aspects of quantizing the open bosonic string is the following. In the free theory, the second term in Eq.(3.39) is dropped. Then the theory is invariant under the tower of gauge transformations $\delta \Lambda_{-n}=Q \Lambda_{-n-1}$ for $n \geq-1$ where $\Lambda_{1} \equiv A$. The gauge invariances of gauge invariances hold off-shell because of the nilpotency of $Q$. So the quantization of the free-theory proceeds without any non-zero on-shell structure tensors. In contrast, for the interacting theory there exist non-zero on-shell tensors in the gauge structure.

Indeed, let us determine the gauge structure of the theory. The algebra is closed because the commutator of two gauge transformations $\Lambda_{0}^{(1)}$ and $\Lambda_{0}^{(2)}$ is equivalent to a single gauge transformation given by $\Lambda_{0}^{(12)}=\Lambda_{0}^{(1)} * \Lambda_{0}^{(2)}-\Lambda_{0}^{(2)} * \Lambda_{0}^{(1)}$. Hence, $E_{\alpha \beta}^{j i}=0$ in Eq.(2.23) and $T_{\alpha \beta}^{\gamma}$ is the matrix element of the $*$ operator among three string states. This is also known as the three-point vertex function and has been computed in refs. [68, 214, 142, 143]. The gauge generators $R_{s}$ in Eq.(2.19) correspond to the covariant derivative operator $\mathcal{D}_{(-s)}$ restricted to the space of $-s$ forms,

$$
R_{s} \leftrightarrow \mathcal{D}_{(-s)},
$$

where $\mathcal{D}_{(q)}$ is defined by

$$
\mathcal{D}_{(q)} B_{q}=Q B_{q}+A * B_{q}-(-1)^{q} B_{q} * A \quad,
$$

when acting on any $q$-form $B_{q}$ and $A$ is the string gauge field. Eq.(2.19), when applied to a $-s$ form ghost $\eta^{(s)}$, reads

$$
\mathcal{D}_{(-s+1)} \mathcal{D}_{(-s)} \eta^{(s)}=F * \eta^{(s)}-\eta^{(s)} * F \quad,
$$

where $F$ is given in Eq.(3.41). Hence, the $V_{s \alpha_{s}}^{i \alpha_{s-2}}$ in Eq.(2.19) are not zero and are given by the commutator of the star operator restricted to the space of $-s$ forms:

$$
S_{0, i} V_{s}^{i} \eta^{(s)} \leftrightarrow F * \eta^{(s)}-\eta^{(s)} * F
$$




\subsection{The Massless Relativistic Spinning Particle}

In this subsection, we consider an example with an anti-commuting gauge parameter, i.e, a system with local supersymmetry. Local supersymmetric theories are also known as supergravity theories. In such theories, the lagrangians and transformation laws are usually complicated. Since our purpose is not to review supersymmetry, we treat one of the simplest examples: the massless relativistic spinning particle [10, 41, 65]. It is a $(0+1)$-dimensional supergravity theory.

We begin with the massless flat-space version of the spinless relativistic particle, namely, the theory discussed in Sect. 3.1 and governed by the action in Eq.(3.1) with $e=1$ and $m=0$. To obtain local supersymmetry, one can proceed in two ways. One can first implement general coordinate invariance and then supersymmetry, or one can first implement supersymmetry and then general coordinate invariance:

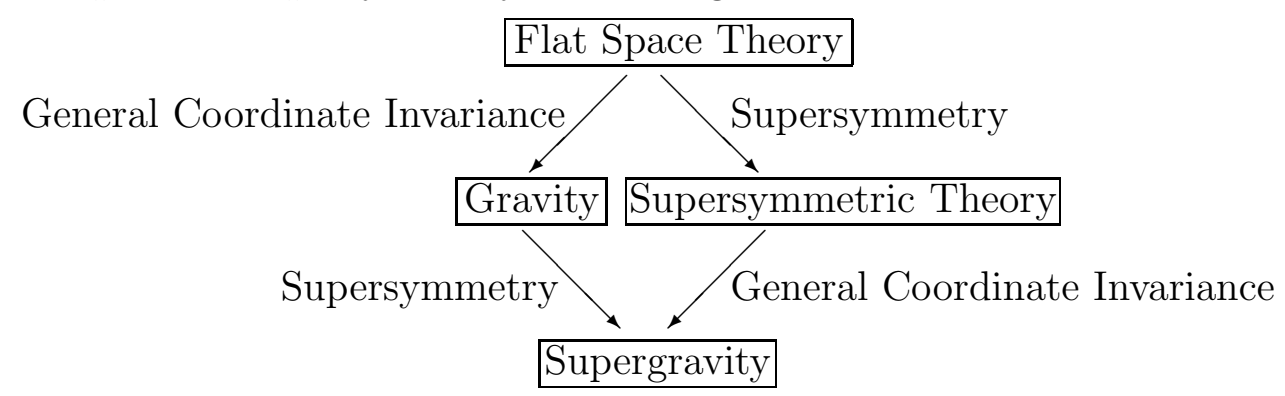

The result of such a program is a supergravity.

Consider the first step of the left path of the above diagram. To implement general coordinate invariance, the Poincaré group must be realized locally. In $0+1$ dimensions, the Poincaré group consists of translations: $\tau \rightarrow \tau+\varepsilon$. The local version of this transformation is $\tau \rightarrow \tau+\varepsilon(\tau)$, that is, $\varepsilon$ becomes a function of $\tau$. The fields for the spinless relativistic particle are the $x^{\mu}(\tau)$ of Sect. 3.1. Under local translations they change as $x^{\mu}(\tau+\varepsilon(\tau))-x^{\mu}(\tau)=\varepsilon(\tau) \dot{x}^{\mu}(\tau)+\ldots$. Hence, the transformation law for $x^{\mu}(\tau)$ is the one given in Eq.(3.6).

As in any gauge theory, it is necessary to introduce a gauge field and promote ordinary derivatives to covariant derivatives 256,276, 177]: $\partial_{\tau} \rightarrow \mathcal{D}_{\tau}$ so that covariant derivatives transform in the same way as fields, namely

$$
\delta\left(\mathcal{D}_{\tau} x^{\mu}(\tau)\right)=\varepsilon(\tau) \partial_{\tau}\left(\mathcal{D}_{\tau} x^{\mu}(\tau)\right)
$$

The gauge field is contained in the einbein $e$ of Sect. 3.1. It transforms as in Eq.(3.6). The covariant time derivative $\mathcal{D}_{\tau}$ is

$$
\mathcal{D}_{\tau} x^{\mu}(\tau) \equiv \frac{1}{e} \partial_{\tau} x^{\mu}(\tau)
$$

Using Eq.(3.6) it is a simple exercise to verify that $\mathcal{D}_{\tau} x^{\mu}(\tau)$ transforms as in Eq.(3.47). 
Any function $F$ of $x^{\mu}$ and $\mathcal{D}_{\tau} x^{\mu}$ transforms in the same way as $x^{\mu}$, i.e.,

$$
\delta F=\varepsilon(\tau) \partial_{\tau} F
$$

as is easily checked. Given any such $F$, the action

$$
\int \mathrm{d} \tau e F\left(x^{\mu}, \mathcal{D}_{\tau} x^{\mu}\right)
$$

is invariant since

$$
\int \mathrm{d} \tau \delta(e F)=\int \mathrm{d} \tau\left(\partial_{\tau}(\varepsilon e) F+\varepsilon e \partial_{\tau} F\right)=\int \mathrm{d} \tau \partial_{\tau}(\varepsilon e F) \longrightarrow 0,
$$

if $\varepsilon(\tau)$ goes to zero at $\tau= \pm \infty$. The action in Eq.(3.1) corresponds to

$$
F=\frac{1}{2}\left(\mathcal{D}_{\tau} x^{\mu}(\tau) \mathcal{D}_{\tau} x_{\mu}(\tau)-m^{2}\right) .
$$

One could also add to Eq.(3.51) an arbitrary potential term $V\left(x^{\mu}(\tau)\right)$ and the theory would still be locally coordinate invariant; however, the global symmetries $x^{\mu} \rightarrow$ $x^{\mu}+a^{\mu}$, where $a^{\mu}$ are constants, would be broken. Requiring such a symmetry forces $V\left(x^{\mu}(\tau)\right)$ to be constant.

The above describes the general idea in constructing theories with general coordinate invariance. In higher-dimensions, $e$ has more components and a spin connection must be introduced for fields with spin. In short, the procedure labelled "general coordinate invariance" in the above diagram corresponds to introducing gravitational gauge fields and promoting ordinary derivatives to covariant derivatives. For more details, see the example in Sect. 3.8 .

Let us consider the process of supersymmetrizing the massless version of the spinless relativistic particle, the example presented in Sect. 3.1. The action is given in Eq.(3.1) with $m=0$ and $e=1$. It is necessary to have $m=0$ because there does not exist a supersymmetric generalization of the $m^{2}$ term. The fields of the spinless relativistic particle are $x^{\mu}$ which are functions of the proper time variable $\tau$. To supersymmetrize the theory, one goes from ordinary space to superspace by enlarging the coordinate system from $\tau$ to $\tau$ and $\theta: \tau \rightarrow(\tau, \theta)$, where the anticommuting coordinate $\theta$ is the supersymmetric partner of $\tau$. Fields are then promoted to superfields by making them functions of $\theta$ :

$$
x^{\mu}(\tau) \rightarrow X^{\mu}(\tau, \theta) \equiv x^{\mu}(\tau)+\theta \psi^{\mu}(\tau),
$$

where the right-hand side of Eq.(3.52) is a Taylor series expansion in $\theta$. It terminates at $\theta$ because $\theta \theta=0$. The $\theta$-component of the superfield $X^{\mu}$ is $\psi^{\mu}$. It is the fermionic partner of $x^{\mu}$. 
Although our purpose is not to review supersymmetry, we present enough background to make this section self-contained. For extensive reviews on supersymmetry see refs. 124, 267. Superfields form an algebra. This means that they can be added and multiplied. Given two superfields $A_{1}=a_{1}+\theta b_{1}$ and $A_{2}=a_{2}+\theta b_{2}$ their sum is $A_{1}+A_{2}=\left(a_{1}+a_{2}\right)+\theta\left(b_{1}+b_{2}\right)$ and their product is $A_{1} A_{2}=a_{1} a_{2}+a_{1} \theta b_{2}+\theta b_{1} a_{2}=$ $a_{1} a_{2}+\theta\left(b_{1} a_{2}+(-1)^{\epsilon\left(a_{1}\right)} a_{1} b_{2}\right)$. Superfields can be manipulated in a manner similar to complex numbers. One only has to be careful about minus signs arising from quantities that anticommute. Supersymmetry calculus is also straightforward. In addition to taking derivatives with respect to $\tau$ one can take derivatives with respect to $\theta$. In a natural way, one defines $\partial_{\theta} \equiv \frac{\partial}{\partial \theta}$ so that $\partial_{\theta} A=b$ when $A=a+\theta b$. Note that $\partial_{\theta}$ is a left derivative. Integration is defined as the operation that selects the $\theta$ component of a superfield and serves the same function as $\partial_{\theta}: \int \mathrm{d} \theta A=\partial_{\theta} A=b$. For more on Grassmann integration and supermanifolds, see refs. 38, 40, 188, 85, 39.

The generator of supersymmetry transformations is denoted by $Q$ (not to be confused with the BRST operator of the previous subsection). It is also convenient to use instead of $\partial_{\theta}$ a generalized $\theta$-derivative $D_{\theta}$. More explicitly $D_{\theta}$ and $Q$ are

$$
\begin{gathered}
D_{\theta} \equiv \partial_{\theta}-i \theta \partial_{\tau} \\
Q \equiv \partial_{\theta}+i \theta \partial_{\tau} .
\end{gathered}
$$

The 1-dimensional super-algebra is

$$
\begin{gathered}
\{Q, Q\}=2 Q Q=2 i \partial_{\tau} \equiv 2 H, \\
{[H, Q]=0 .}
\end{gathered}
$$

These equations are easily verified using Eq.(3.53). In theories for which a supersymmetry charge $Q$ exists, the hamiltonian necessarily has a non-negative spectrum as a consequence of $H=Q Q \geq 0$. In addition, $[H, Q]=0$ implies that there is a fermionic state for every bosonic state and vice-versa, except possibly for a zero energy state. The (anti)commutators involving $D_{\theta}$ are $\left\{Q, D_{\theta}\right\}=0,\left[H, D_{\theta}\right]=0$, and $\left\{D_{\theta}, D_{\theta}\right\}=-2 H$.

The supersymmetry transformations are defined by $\delta A=i \xi Q A$ where $\xi$ is an anticommuting parameter. For $X^{\mu}$, a simple calculation gives $\delta X^{\mu}=i \xi Q X^{\mu}=$ $i \xi \psi^{\mu}+\theta \xi \partial_{\tau} x^{\mu}$. Given that $\delta X^{\mu}$ is defined by $\delta X^{\mu} \equiv \delta x^{\mu}+\theta \delta \psi^{\mu}$, one obtains for the transformation rules for the components of $X^{\mu}$

$$
\begin{gathered}
\delta x^{\mu}=i \xi \psi^{\mu} \\
\delta \psi^{\mu}=\xi \partial_{\tau} x^{\mu}
\end{gathered} .
$$


The reason for using $D_{\theta}$ in lieu of $\partial_{\theta}$ is that $D_{\theta} A$ transforms as a superfield if $A$ transforms as such, since $D_{\theta}$ commutes with $Q: \delta\left(D_{\theta} A\right)=D_{\theta}(i \xi Q A)=i \xi Q\left(D_{\theta} A\right)$. The same statement holds for $\partial_{\tau} A$ since $\left[\partial_{\tau}, Q\right]=-i[H, Q]=0$ so that one does not need to generalize $\partial_{\tau}$.

Supersymmetric-invariant actions are constructed from the $\theta$ component of a superfield: Note that $\delta\left(\left.A\right|_{\theta \text { component }}\right)=\xi \partial_{\tau}\left(\left.A\right|_{\theta=0}\right)$ so that if one integrates this component over $\tau$ to obtain an action $S_{0}$, this action is invariant if fields fall off sufficiently fast at $\tau \rightarrow \pm \infty$. Given that $\partial_{\tau} X^{\mu}, D_{\theta} X^{\mu}$ and $X^{\mu}$ are all superfields and that superfields form an algebra, the $\theta$ component of any function of these fields transforms as a total $\tau$ derivative: $\delta\left(\left.F\left(\partial_{\tau} X^{\mu}, D_{\theta} X^{\mu}, X^{\mu}\right)\right|_{\theta \text { component }}\right)=\xi \partial_{\tau}\left(\left.F\right|_{\theta=0}\right)$. Since $\int \mathrm{d} \theta$ picks out the $\theta$ component of a superfield, an invariant action is

$$
S_{0}=\int \mathrm{d} \tau \int \mathrm{d} \theta F\left(\partial_{\tau} X^{\mu}, D_{\theta} X^{\mu}, X^{\mu}\right)
$$

The supersymmetric generalization of the action for the massless relativistic particle consists in taking $F$ in (3.56) as $F=\frac{i}{2} D_{\theta} X^{\mu} \partial_{\tau} X_{\mu}$ since, when written in component fields, it contains the correct kinetic energy term for $x^{\mu}$ :

$$
S_{0}=\frac{i}{2} \int \mathrm{d} \tau \int \mathrm{d} \theta D_{\theta} X^{\mu} \partial_{\tau} X_{\mu}=\frac{1}{2} \int \mathrm{d} \tau\left(\partial_{\tau} x^{\mu} \partial_{\tau} x_{\mu}-i \psi^{\mu} \partial_{\tau} \psi_{\mu}\right) \quad .
$$

It is easily checked that Eq.(3.57) is invariant under the transformations in Eq.(3.55). One could add interactions by using a more general $F\left(\partial_{\tau} X^{\mu}, D_{\theta} X^{\mu}, X^{\mu}\right)$.

The final goal of this subsection is to implement both local translational and local supersymmetry and construct a supergravity theory. Following the procedures above, we promote the einbein $e(\tau)$ to a superfield $E: e(\tau) \rightarrow E(\tau, \theta)$ where

$$
E(\tau, \theta) \equiv e(\tau)+\theta \chi(\tau)
$$

Here, $\chi$ is the superpartner of $e$. The infinitesimal gauge parameters associated with general coordinate invariance and supersymmetry are grouped into one superfield $\eta^{t}$ via

$$
\eta^{t}(\tau, \theta) \equiv \varepsilon(\tau)+\theta \xi(\tau)
$$

Using $\eta^{t}$ and the $\theta$-partner to $\eta^{t}$, given by

$$
\eta^{\theta}(\tau, \theta) \equiv i E \partial_{\theta} \eta^{t}=i e \xi(\tau)+i \theta \chi \xi(\tau)
$$

one writes the transformation rule for a superfield such as $A(\tau, \theta)$ as

$$
\delta A=\eta^{t}(\tau, \theta) \partial_{\tau} A+\eta^{\theta}(\tau, \theta) \partial_{\theta} A .
$$

Notice that Eq. 3.61) reduces to the correct transformation law $\delta A=\varepsilon(\tau) \partial_{\tau} A$ when $\xi=0$. Furthermore, when $\chi \rightarrow 0, \varepsilon(\tau) \rightarrow 0, e \rightarrow 1$, and $\xi$ is not longer a function of 
$\tau$, the right-hand side of Eq.(3.61) becomes $\left(i \xi \partial_{\theta}+\theta \xi \partial_{\tau}\right) A=i \xi Q A$, which is the flat space supersymmetry transformation. Hence Eq.(3.61) reduces to expected results in these two limits. The transformation law for $E$ must be generalized from $\delta e=\partial_{\tau}(\varepsilon e)$. As will become clear below when we construct invariant actions, one needs

$$
\delta E=\partial_{\tau}\left(\eta^{t} E\right)-\partial_{\theta}\left(\eta^{\theta} E\right) .
$$

With this transformation law for $E$, it is straightforward to find covariant derivative operators $\mathcal{D}_{\tau}$ and $\mathcal{D}_{\theta}$ that maintain the supersymmetry transformation law in Eq.(3.61). One requires $\delta\left(\mathcal{D}_{\tau} A\right)=\eta^{t} \partial_{\tau}\left(\mathcal{D}_{\tau} A\right)+\eta^{\theta} \partial_{\theta}\left(\mathcal{D}_{\tau} A\right)$ and $\delta\left(\mathcal{D}_{\theta} A\right)=$ $\eta^{t} \partial_{\tau}\left(\mathcal{D}_{\theta} A\right)+\eta^{\theta} \partial_{\theta}\left(\mathcal{D}_{\theta} A\right)$. A solution is

$$
\begin{gathered}
\mathcal{D}_{\tau} A \equiv \frac{1}{E}\left(\partial_{\tau} A-i \partial_{\theta} E \partial_{\theta} A\right) \\
\mathcal{D}_{\theta} A \equiv \partial_{\theta} A-i \theta \mathcal{D}_{\tau} A .
\end{gathered}
$$

When acting on the superfield $X^{\mu}$, one finds

$$
\begin{gathered}
\mathcal{D}_{\tau} X^{\mu}=\frac{1}{e}\left(\partial_{\tau} x^{\mu}-i \chi \psi^{\mu}\right)+\frac{\theta}{e}\left(\partial_{\tau} \psi^{\mu}-\frac{\chi}{e} \partial_{\tau} x^{\mu}\right), \\
\mathcal{D}_{\theta} X^{\mu}=\psi^{\mu}-i \frac{\theta}{e}\left(\partial_{\tau} x^{\mu}-i \chi \psi^{\mu}\right) .
\end{gathered}
$$

Again, superfields form an algebra so that any function $F$ of $\mathcal{D}_{\tau} X^{\mu}, \mathcal{D}_{\theta} X^{\mu}$ and $X^{\mu}$ transforms as a superfield in curved space:

$$
\delta F\left(\mathcal{D}_{\tau} X^{\mu}, \mathcal{D}_{\theta} X^{\mu}, X^{\mu}\right)=\eta^{t} \partial_{\tau} F+\eta^{\theta} \partial_{\theta} F \quad .
$$

It is now straightforward to show that

$$
S_{0}=\int \mathrm{d} \tau \int \mathrm{d} \theta E F
$$

is an invariant action since

$$
\begin{gathered}
\delta \int \mathrm{d} \tau \int \mathrm{d} \theta E F= \\
\int \mathrm{d} \tau \int \mathrm{d} \theta\left(\left(\partial_{\tau}\left(\eta^{t} E\right)-\partial_{\theta}\left(\eta^{\theta} E\right)\right) F+E\left(\eta^{t} \partial_{\tau} F+\eta^{\theta} \partial_{\theta} F\right)\right) \\
=\int \mathrm{d} \tau \int \mathrm{d} \theta\left(\partial_{\tau}\left(\eta^{t} E F\right)-\partial_{\theta}\left(\eta^{\theta} E F\right)\right)
\end{gathered}
$$

is a total derivative. The transformation rule for $E$ in Eq.(3.62), including the minus sign on the right-hand side, was deliberately chosen so that $E F$ would lead to 
an invariant super-lagrangian. Taking $F=\frac{i}{2} \mathcal{D}_{\theta} X^{\mu} \mathcal{D}_{\tau} X_{\mu}$ produces the extension of Eq.(3.57) with general coordinate invariance:

$$
\begin{gathered}
S_{0}=\frac{i}{2} \int \mathrm{d} \tau \int \mathrm{d} \theta E \mathcal{D}_{\theta} X^{\mu} \mathcal{D}_{\tau} X_{\mu}= \\
\frac{1}{2} \int \mathrm{d} \tau\left(\frac{1}{e} \partial_{\tau} x^{\mu} \partial_{\tau} x_{\mu}-i \psi^{\mu} \partial_{\tau} \psi_{\mu}-\frac{2 i \chi}{e} \psi^{\mu} \partial_{\tau} x_{\mu}\right) .
\end{gathered}
$$

By construction, one is guaranteed that Eq.(3.66) is invariant under the transformations in Eqs.(3.61) (when $A=X^{\mu}$ ) and (3.62). In components, these equations read

$$
\begin{gathered}
\delta x^{\mu}=\varepsilon \partial_{\tau} x^{\mu}+i e \xi \psi^{\mu} \\
\delta \psi^{\mu}=\varepsilon \partial_{\tau} \psi^{\mu}+\xi\left(\partial_{\tau} x^{\mu}-i \chi \psi^{\mu}\right) \\
\delta e=\partial_{\tau}(\varepsilon e)+2 i e \xi \chi \\
\delta \chi=\partial_{\tau}(\varepsilon \chi+\xi e)
\end{gathered}
$$

A good exercise is to check explicitly that Eq.(3.66) is invariant under Eq.(3.67).

Since the transformations rules in Eqs.(3.61) and (3.62) are defined independent of an action, the algebra should close off-shell. The only thing to check is that the commutator of two transformations produces an effect on $E$ similar to the one on $X^{\mu}$. A little algebra reveals that

$$
\left[\delta_{1}, \delta_{2}\right] \phi^{i}=\delta_{12} \phi^{i}
$$

where $\phi^{i}=x^{\mu}, \psi^{\mu}, e$ or $\chi$, and $\delta_{12}$ corresponds to a transformation with

$$
\begin{gathered}
\varepsilon_{12}=\varepsilon_{2} \dot{\varepsilon}_{1}-\varepsilon_{1} \dot{\varepsilon}_{2}+2 i e \xi_{2} \xi_{1} \\
\xi_{12}=\varepsilon_{2} \dot{\xi}_{1}-\varepsilon_{1} \dot{\xi}_{2}+\dot{\varepsilon}_{1} \xi_{2}-\dot{\varepsilon}_{2} \xi_{1}+2 i \chi \xi_{2} \xi_{1}
\end{gathered}
$$

The algebra closes off-shell but has field-dependent structure constants due to the presence of $e$ and $\xi$ in Eq.(3.69). In the non-supersymmetric limit for which $\xi_{1}=$ $\xi_{2}=\chi=0$, the spinless-relativistic-particle algebra in Eq.(3.7) is reproduced. In the flat-space limit for which $\chi=0, e=1$, and gauge parameters do not depend on $\tau$, Eq.(3.69) reduces to the flat space supersymmetry algebra in Eq.(3.54).

In four dimensions, the construction of supergravity theories is considerably more complicated. Several gravitational superfields enter. The interested reader can find introductions to four-dimensional supergravity in refs. 258, 124, 267]. The formalism presented here is the one-dimensional analog of the covariant- $\Theta$ formalism of Chapters XX and XXI of ref.267. 


\subsection{The First-Quantized Bosonic String}

The open bosonic string is the two-dimensional generalization of the relativistic particle considered in Sect. 3.1. It is a general-coordinate-invariant theory. As such, it can be regarded as a certain type of two-dimensional gravity theory.

Let us review the construction of general-coordinate-invariant theories involving some scalar fields $A^{i}$. In what follows, all the $A^{i}$ behave similarly and we drop the superscript index $i$. We use the vielbein formulation. The gauge fields which implement local coordinate invariances are vielbeins $e_{a}{ }^{m}$, where $a=0, \ldots, d-1$ and $m=0, \ldots, d-1$. These indices take on $d$ values corresponding to time and space coordinates. The index $m$ is the Einstein index associated with local coordinate transformations. The index $a$ is the tangent-space Lorentz index used for implementing local Lorentz transformations. The inverse metric $g^{m n}$ is the inverse of the metric $g_{m n}: g^{m p} g_{p n} \equiv \delta_{n}^{m}$. It is related to the vielbein via $g^{m n} \equiv \eta^{a b} e_{a}^{m} e_{b}^{n}$, where $\eta^{a b}$ is the flat-space metric. For $d=2, \eta^{a b}=\eta_{a b}=\left(\begin{array}{cc}-1 & 0 \\ 0 & 1\end{array}\right)$. Lorentz indices $a, b, c$, etc. are raised and lowered using $\eta^{a b}$ and $\eta_{a b}$. Einstein indices are raised and lowered using $g^{m n}$ and $g_{m n}$. Hence, the inverse vielbein $e^{a}{ }_{m}$ is $e^{a}{ }_{m} \equiv g_{m n} \eta^{a b} e_{b}{ }^{n}$ and satisfies $e^{a}{ }_{n} e_{a}{ }^{m}=\delta_{n}^{m}$ and $e^{a}{ }_{m} e_{b}{ }^{m}=\delta_{b}^{a}$, as one can easily check. The metric can be expressed in terms of $e^{a}{ }_{m}$ via $g_{m n}=\eta_{a b} e^{a}{ }_{m} e^{b}{ }_{n}$.

Under an infinitesimal local translation in the $m$-th direction by $\varepsilon^{m}$, a scalar field $A$ changes by $\delta A=A\left(x^{m}+\varepsilon^{m}\right)-A\left(x^{m}\right)$ or

$$
\delta A=\varepsilon^{m} \partial_{m} A
$$

One would like to find covariant derivatives $\mathcal{D}_{a} A$ that transform in the same way: $\delta\left(\mathcal{D}_{a} A\right)=\varepsilon^{m} \partial_{m}\left(\mathcal{D}_{a} A\right)$. The vielbein $e_{a}{ }^{m}$ allows one to do this. Letting

$$
\mathcal{D}_{a} A=e_{a}^{m} \partial_{m} A
$$

and requiring the correct transformation law for $\mathcal{D}_{a} A$, one finds that $e_{a}{ }^{m}$ must transform as

$$
\delta e_{a}^{m}=\varepsilon^{n} \partial_{n} e_{a}^{m}-e_{a}^{n} \partial_{n} \varepsilon^{m} .
$$

Any function $F$ of $A$ and $\mathcal{D}_{a} A$ will transform like $A: \delta F\left(A, \mathcal{D}_{a} A\right)=\varepsilon^{m} \partial_{m} F$. Suppose one can find a density $e$ whose transformation law is

$$
\delta e=\partial_{m}\left(\varepsilon^{m} e\right)
$$

Then $e F$ transforms as a total derivative $\delta e F=\partial_{m}\left(\varepsilon^{m} e F\right)$ and is a candidate for an action density. When the action density is integrated over space-time, it is invariant under local coordinate transformations as long as gauge parameters vanish at infinity. 
The density $e$ can be constructed from the determinant of the vielbein $\operatorname{det}(e \cdot)$. Note that $\operatorname{det}(e:)$ transforms as

$$
\delta \operatorname{det}\left(e_{:}:\right)=\delta e_{a}{ }^{m} \times \text { cofactor matrix of } e_{a}{ }^{m}=\delta e_{a}{ }^{m} e^{a}{ }_{m} \operatorname{det}\left(e^{\cdot}\right)
$$

The derivative of $\operatorname{det}(e:)$ is

$$
\partial_{n} \operatorname{det}\left(e^{\cdot}\right)=\partial_{n} e_{a}{ }^{m} \times \text { cofactor matrix of } e_{a}{ }^{m}=\partial_{n} e_{a}{ }^{m} e^{a}{ }_{m} \operatorname{det}\left(e_{:}\right) \text {. }
$$

Using these results, a short calculation reveals that

$$
e \equiv \frac{1}{\operatorname{det}(e \cdot)}=\operatorname{det}\left(e^{*}\right)
$$

transforms as in Eq.(3.73). The relativistic particle, presented as the example in Sect. 3.1, is a $(0+1)$-dimensional theory where the field $e$ in that section is the same as the density $e$ defined here: $e=\operatorname{det}\left(e^{:}\right)=e^{0}{ }_{0}=1 / e_{0}{ }^{0}$.

In contrast to the metric $g_{m n}$, which has $d(d+1) / 2$ degrees of freedom, the vielbein $e_{a}{ }^{m}$ has $d^{2}$ degrees of freedom. To compensate for this difference, one requires that the theory be invariant under local Lorentz transformations that act on Lorentz indices $a, b$, etc.. The transformation laws are

$$
\delta e_{a}{ }^{m}=(\varepsilon M)_{a}{ }^{b} e_{b}{ }^{m}, \quad \delta A=0,
$$

where $(\varepsilon M)_{a}{ }^{b}$ stands for $\frac{1}{2}\left(\varepsilon^{c d} M_{c d}\right)_{a}{ }^{b}$. Here, the $d(d-1) / 2$ parameters $\varepsilon^{c d}$ satisfy $\varepsilon^{c d}=$ $-\varepsilon^{d c}$ and characterize the size of the infinitesimal-local-Lorentz transformations. The $d(d-1) / 2$ matrix generators $M_{a b}$ satisfy the Lorentz algebra $\left[M_{a b}, M_{c d}\right]=-\eta_{a c} M_{b d}+$ $\eta_{a d} M_{b c}+\eta_{b c} M_{a d}-\eta_{b d} M_{a c}$ and are antisymmetric in the lower indices $c$ and $d: M_{c d}=$ $-M_{d c}$. When we write $\left(M_{c d}\right)_{a}{ }^{b}, a$ and $b$ are matrix indices whereas $c$ and $d$ label the different Lorentz transformations: $M_{c d}$ produces an infinitesimal transformation in the $c-d$ plane. One explicit realization of $M_{c d}$ is $\left(M_{c d}\right)_{a}{ }^{b}=\eta_{a c} \delta_{d}^{b}-\eta_{a d} \delta_{c}^{b}$. The extra $d(d-1) / 2$ gauge invariances guarantees that the physical numbers of degrees of freedom in $e_{a}{ }^{m}$ and $g_{m n}$ are the same.

Given a number of fields $V_{a}^{i}$, with Lorentz indices, local invariants are constructed by using the flat space metric $\eta^{a b}$ via $\eta^{a b} V_{a}^{i} V_{b}^{j}$ or the flat space antisymmetric $\varepsilon^{a_{0} a_{1} \ldots a_{d-1}}$ symbol via $\varepsilon^{a_{0} a_{1} \ldots a_{d-1}} V_{a_{0}}^{i_{0}} V_{a_{1}}^{i_{1}} \ldots V_{a_{d-1}}^{i_{d-1}}$. A locally general coordinate and Lorentz invariant action for a scalar field $A$ is

$$
S_{0}=-\frac{1}{2} \int \mathrm{d} t \mathrm{~d}^{d-1} x e \eta^{a b} \mathcal{D}_{a} A \mathcal{D}_{b} A
$$

For the rest of this subsection, we set $d=2$. When the dimension of spacetime is $1+1$, the action Eq.(3.76) is also classically invariant under local conformal transformations that scale the vielbein by a spacetime dependent function $\varepsilon$ :

$$
\delta e_{a}{ }^{m}=\varepsilon e_{a}{ }^{m}, \quad \delta A=0 .
$$


We thus have three types of local transformations: translations, Lorentz rotations and scaling. Let us respectively associate $\varepsilon^{*}, \varepsilon^{\prime \prime}$ and $\varepsilon$ with each of these. In other words, we use the number of indices on the infinitesimal parameter to distinguish the transformations. The algebra of gauge transformations is as follows. The commutator of two local translations is a local translation so that they form a subalgebra. Likewise the commutator of two local Lorentz transformations is a local Lorentz transformation. The commutator of a translation and a Lorentz rotation is a Lorentz rotation. Local translations and Lorentz rotations also form a subalgebra. Local scaling transformations commute. They also commute with local Lorentz transformations. Finally, the commutator of a scaling transformation and a translation produces a scaling transformation. These last few statements correspond to

$$
\begin{gathered}
{\left[\delta\left(\varepsilon_{1}^{*}\right), \delta\left(\varepsilon_{2}\right)\right]=\delta\left(\varepsilon_{12}\right), \quad \text { where } \varepsilon_{12}^{m}=\varepsilon_{2}^{n} \partial_{n} \varepsilon_{1}^{m}-\varepsilon_{1}^{n} \partial_{n} \varepsilon_{2}^{m},} \\
{\left[\delta\left(\varepsilon_{1}^{*}\right), \delta\left(\varepsilon_{2}^{*}\right)\right]=\delta\left(\varepsilon_{12}^{*}\right), \quad \text { where } \varepsilon_{12}^{a b}=\left(\varepsilon_{1}^{a c} \varepsilon_{2}^{b d}-\varepsilon_{2}^{a c} \varepsilon_{1}^{b d}\right) \eta_{c d},} \\
{\left[\delta\left(\varepsilon_{1}^{*}\right), \delta\left(\varepsilon_{2}^{*}\right)\right]=\delta\left(\varepsilon_{12}^{*}\right), \quad \text { where } \varepsilon_{12}^{a b}=\varepsilon_{2}^{n} \partial_{n} \varepsilon_{1}^{a b},} \\
{\left[\delta\left(\varepsilon_{1}\right), \delta\left(\varepsilon_{2}\right)\right]=0,} \\
{\left[\delta\left(\varepsilon_{1}\right), \delta\left(\varepsilon_{2}^{*}\right)\right]=0,} \\
{\left[\delta\left(\varepsilon_{1}\right), \delta\left(\varepsilon_{2}\right)\right]=\delta\left(\varepsilon_{12}\right), \quad \text { where } \quad \varepsilon_{12}=\varepsilon_{2}^{n} \partial_{n} \varepsilon_{1} .}
\end{gathered}
$$

Equation (3.78) is the gauge algebra for the bosonic string. It is a closed irreducible algebra with field-independent commutator structure constants and hence a Lie algebra.

The scalar fields for the $D=26$ bosonic string are target space coordinate $X^{\mu}$ where $\mu$ ranges from 0 to 25 . The action is Eq.(3.76) for each of these fields

$$
S_{0}=-\frac{1}{2} \int \mathrm{d} \tau \int_{0}^{\pi} \mathrm{d} \sigma e \eta^{a b} \eta_{\mu \nu} \mathcal{D}_{a} X^{\mu} \mathcal{D}_{b} X^{\nu},
$$

where $\eta_{\mu \nu}$ is the flat-space $25+1$ metric with signature $(-1,1,1, \ldots, 1), \mathcal{D}_{a}$ is given in Eq.(3.71), and $e=1 /\left(e_{\tau}{ }^{\tau} e_{\sigma}{ }^{\sigma}-e_{\tau}{ }^{\sigma} e_{\sigma}{ }^{\tau}\right)$. The world-sheet coordinates are denoted by $\tau$ and $\sigma$ instead of $t$ and $x$. Hence the first-quantized bosonic string is a scale-invariant two-dimensional gravity theory on a finite spatial region since $\sigma$ ranges from 0 to $\pi$. For the closed bosonic string, the fields $X^{\mu}$ are periodic in $\sigma$, whereas for the open bosonic string, one requires $\partial_{\sigma} X^{\mu}$ to vanish at $\sigma=0$ and $\sigma=\pi$. Eq.(3.79) is invariant under the general coordinate transformations in Eqs.(3.70) and (3.72), under the Lorentz gauge transformations in Eq.(3.75), and under the local scale transformations in Eq.(3.77) where $A$ stands for $X^{\mu}$; however, the local translation parameters $\varepsilon^{m}$ must obey the same boundary conditions as $X^{\mu}$. It is a useful exercise to check explicitly the gauge invariances of Eq.(3.79). 
There are four gauge invariances, two translations, one Lorentz boost and one scaling transformation. It is necessary for the target space to be 26 dimensions in order to avoid an anomaly in one of these invariances. This is discussed in Sect. 9.3. Hence in $D=26$, the components of $e_{a}{ }^{m}$ can be fixed to constant values. Often $e_{a}{ }^{m}=\left(\begin{array}{ll}1 & 0 \\ 0 & 1\end{array}\right)$ is used. Then Eq.(3.79) becomes a free field theory. However, in gauge fixing, one should introduce ghosts. These ghosts play a somewhat minor role in the first-quantized theory. However, in the second-quantized formulation, namely the string field theory of Sect. 3.6, the ghosts as well as $X^{\mu}(\sigma)$ become coordinates of the string field $A$ in Eq.(3.39).

Eq.(3.79) is classically equivalent to the usual Nambu-Goto action [197, [38]. Using the equations of motion of $e_{a}{ }^{m}$ to eliminate the vielbein, one finds, after some algebra, that

$$
\frac{1}{2} e \eta^{a b} \eta_{\mu \nu} \mathcal{D}_{a} X^{\mu} \mathcal{D}_{b} X^{\nu} \rightarrow \sqrt{\left(\partial_{\tau} X^{\mu} \partial_{\sigma} X_{\mu}\right)^{2}-\left(\partial_{\tau} X^{\mu} \partial_{\tau} X_{\mu}\right)\left(\partial_{\sigma} X^{\nu} \partial_{\sigma} X_{\nu}\right)},
$$

which is the familiar Nambu-Goto action density. 


\section{The Field-Antifield Formalism}

Consider the classical system defined in Sect. 2, described by the action $S_{0}\left[\phi^{i}\right]$ and having gauge invariances. The ultimate goal is to quantize this theory in a covariant way. The field-antifield formalism was developed to achieve this aim. In this section, we present the field-antifield formalism at the classical level. In brief, it involves five steps:

(i) The original configuration space, consisting of the $\phi^{i}$, is enlarged to include additional fields such as ghost fields, ghosts for ghosts, etc.. One also introduces the antifields of these fields.

(ii) On the space of fields and antifields, one defines an odd symplectic structure $($, ) called the antibracket.

(iii) The classical action $S_{0}$ is extended to include terms involving ghosts and antifields and is denoted by $S$.

(iv) The classical master equation is defined to be $(S, S)=0$.

(v) Finally, one finds solutions to the classical master equation subject to certain boundary conditions.

The key result of this section is that the solution in steps (iv) and (v) leads to a set of equations containing all relations defining the gauge algebra and its solution. The action $S$ is the generating functional for the structure functions. Hence, the fieldantifield formalism is a compact and efficient way of obtaining the gauge structure derived in Sect. 2 .

\subsection{Fields and Antifields}

Suppose a theory is irreducible with $m_{0}$ gauge invariances. Then, at the quantum level, $m_{0}$ ghost fields are needed. As in Sect. 2.4, it is useful to introduce these ghost fields at the classical level. Hence, the field set $\Phi^{A}$ is $\Phi^{A}=\left\{\phi^{i}, \mathcal{C}_{0}^{\alpha_{0}}\right\}$ where $\alpha_{0}=1, \ldots, m_{0}$. If the theory is first-stage reducible, there are gauge invariances for gauge invariances and hence there are ghosts for ghosts. If there are $m_{1}$ first-level

gauge invariances then, to the above set, one adds the ghost-for-ghost fields $\mathcal{C}_{1}^{\alpha_{1}}$ where $\alpha_{1}=1, \ldots, m_{1}$. In general for an $L$-th stage reducible theory, the set of fields $\Phi^{A}$, $A=1, \ldots, N$, is

$$
\Phi^{A}=\left\{\phi^{i}, \mathcal{C}_{s}^{\alpha_{s}} ; s=0, \ldots, L ; \quad \alpha_{s}=1, \ldots, m_{s}\right\}
$$


An additive conserved charge, called ghost number, is assigned to each of these fields. The classical fields $\phi^{i}$ are assigned ghost number zero, whereas ordinary ghosts have ghost number one. Ghosts for ghosts, i.e., level-one ghosts, have ghost number two, etc.. Similarly, ghosts have opposite statistics of the corresponding gauge parameter, but ghosts for ghosts have the same statistics as the gauge parameter, and so on, with the statistics alternating for higher-level ghosts. More precisely,

$$
\operatorname{gh}\left[\mathcal{C}_{s}^{\alpha_{s}}\right]=s+1, \quad \epsilon\left(\mathcal{C}_{s}^{\alpha_{s}}\right)=\epsilon_{\alpha_{s}}+s+1(\bmod 2)
$$

Next, one introduces an antifield $\Phi_{A}^{*}, A=1, \ldots, N$, for each field $\Phi^{A}$. The ghost number and statistics of $\Phi_{A}^{*}$ are

$$
\operatorname{gh}\left[\Phi_{A}^{*}\right]=-\operatorname{gh}\left[\Phi^{A}\right]-1, \quad \epsilon\left(\Phi_{A}^{*}\right)=\epsilon\left(\Phi^{A}\right)+1(\bmod 2)
$$

so that the statistics of $\Phi_{A}^{*}$ is opposite to that of $\Phi^{A}$.

At this stage, the antifields do not have any direct physical meaning. They are only used as a mathematical tool to develop the formalism. However, they can be interpreted as source coefficients for BRST transformations. This is made clear from the discussion of the effective action. See Sect. 8.4. For computing correlation functions, $S$-matrix elements and certain quantities, antifields are eliminated by a gauge-fixing procedure.

The set of fields and antifields just introduced is called the classical basis. [5 When gauge-fixing is considered in Sect. 6, another basis, known as the gauge-fixed basis, can be introduced. Then, the implications of the classical master equation change. This is discussed in Sect. 6.6.

\subsection{The Antibracket}

In the space of fields and antifields, the antibracket ref. 277, 24] is defined by

$$
(X, Y) \equiv \frac{\partial_{r} X}{\partial \Phi^{A}} \frac{\partial_{l} Y}{\partial \Phi_{A}^{*}}-\frac{\partial_{r} X}{\partial \Phi_{A}^{*}} \frac{\partial_{l} Y}{\partial \Phi^{A}}
$$

Many properties of $(X, Y)$ are similar to a graded version of the Poisson bracket, with the grading of $X$ and $Y$ being $\epsilon_{X}+1$ and $\epsilon_{Y}+1$ instead of $\epsilon_{X}$ and $\epsilon_{Y}$. The antibracket satisfies

$$
\begin{gathered}
(Y, X)=-(-1)^{\left(\epsilon_{X}+1\right)\left(\epsilon_{Y}+1\right)}(X, Y), \\
((X, Y), Z)+(-1)^{\left(\epsilon_{X}+1\right)\left(\epsilon_{Y}+\epsilon_{Z}\right)}((Y, Z), X)+(-1)^{\left(\epsilon_{Z}+1\right)\left(\epsilon_{X}+\epsilon_{Y}\right)}((Z, X), Y)=0, \\
\operatorname{gh}[(X, Y)]=\operatorname{gh}[X]+\operatorname{gh}[Y]+1,
\end{gathered}
$$

\footnotetext{
3 This definition differs somewhat from the one of refs. [252, 257].
} 


$$
\epsilon[(X, Y)]=\epsilon_{X}+\epsilon_{Y}+1(\bmod 2) .
$$

The first equation says that $($,$) is graded antisymmetric. The second equation shows$ that $($, ) satisfies a graded Jacobi identity. The antibracket "carries" ghost number one and has odd statistics. From these properties and the definition of right and left derivatives, one concludes that

$$
\begin{gathered}
(B, B)=2 \frac{\partial_{r} B}{\partial \Phi^{A}} \frac{\partial_{l} B}{\partial \Phi_{A}^{*}}, \quad \text { for } B \text { bosonic }, \\
(F, F)=0, \quad \text { for } F \text { fermionic }, \\
((X, X), X)=0, \quad \text { for any } X .
\end{gathered}
$$

The above expression for $(B, B)$ is derived in Appendix A. The antibracket $(X, Y)$ is also a graded derivation with ordinary statistics for $X$ and $Y$ :

$$
\begin{aligned}
& (X, Y Z)=(X, Y) Z+(-1)^{\epsilon_{Y} \epsilon_{Z}}(X, Z) Y, \\
& (X Y, Z)=X(Y, Z)+(-1)^{\epsilon_{X} \epsilon_{Y}} Y(X, Z) .
\end{aligned}
$$

The antibracket defines an odd symplectic structure because it can be written as

$$
(X, Y)=\frac{\partial_{r} X}{\partial z^{a}} \zeta^{a b} \frac{\partial_{l} Y}{\partial z^{b}}, \quad \text { where } \quad \zeta^{a b} \equiv\left(\begin{array}{cc}
0 & \delta_{B}^{A} \\
-\delta_{B}^{A} & 0
\end{array}\right) \quad,
$$

when one groups the fields and antifields collectively into $z^{a}: z^{a}=\left\{\Phi^{A}, \Phi_{A}^{*}\right\}, a=$ $1, \ldots, 2 N$. The expression for the antibracket in Eq.(4.8) is sometimes useful in abstract proofs. The antibracket formalism can be developed in an arbitrary coordinate system, in which case $\zeta^{a b}$ is replaced by an odd closed field-dependent two-form. For more details, see Sect. 10.7. However, locally there always exists a basis for which $\zeta^{a b}$ is of the form of Eq. (4.8) [218].

The antibracket in the space of fields and antifields plays a role analogous to the Poisson bracket. Whereas the Poisson bracket is used at the classical level in a hamiltonian formulation, the antibracket is used at the classical or quantum level in a lagrangian formalism. One can use the antibracket in a manner similar to the Poisson bracket. The antifield $\Phi_{A}^{*}$ can be thought of as the conjugate variable to $\Phi^{A}$ since

$$
\left(\Phi^{A}, \Phi_{B}^{*}\right)=\delta_{B}^{A}
$$

Infinitesimal canonical transformations [24] in which $\Phi^{A} \rightarrow \bar{\Phi}^{A}$ and $\Phi_{A}^{*} \rightarrow \bar{\Phi}_{A}^{*}$, where $\bar{\Phi}^{A}$ and $\bar{\Phi}_{A}^{*}$ are functions of the $\Phi$ and $\Phi^{*}$, are defined by

$$
\bar{\Phi}^{A}=\Phi^{A}+\varepsilon\left(\Phi^{A}, F\right)+O\left(\varepsilon^{2}\right), \quad \bar{\Phi}_{A}^{*}=\Phi_{A}^{*}+\varepsilon\left(\Phi_{A}^{*}, F\right)+O\left(\varepsilon^{2}\right),
$$


where $F$ is an arbitrary function of the fields and antifields with $\operatorname{gh}[F]=-1$ and $\epsilon(F)=1$. A short calculation reveals that the canonical structure in Eq.(4.9) is maintained:

$$
\left(\bar{\Phi}^{A}, \bar{\Phi}_{B}^{*}\right)=\delta_{B}^{A}+O\left(\varepsilon^{2}\right), \quad\left(\bar{\Phi}^{A}, \bar{\Phi}^{B}\right)=0+O\left(\varepsilon^{2}\right), \quad\left(\bar{\Phi}_{A}^{*}, \bar{\Phi}_{B}^{*}\right)=0+O\left(\varepsilon^{2}\right)
$$

Canonical transformations preserve the antibracket, i.e.,

$$
\frac{\partial_{r} X}{\partial \bar{\Phi}^{A}} \frac{\partial_{l} Y}{\partial \bar{\Phi}_{A}^{*}}-\frac{\partial_{r} X}{\partial \bar{\Phi}_{A}^{*}} \frac{\partial_{l} Y}{\partial \bar{\Phi}^{A}}=\frac{\partial_{r} X}{\partial \Phi^{A}} \frac{\partial_{l} Y}{\partial \Phi_{A}^{*}}-\frac{\partial_{r} X}{\partial \Phi_{A}^{*}} \frac{\partial_{l} Y}{\partial \Phi^{A}}+O\left(\varepsilon^{2}\right) \quad .
$$

Under Eq.(4.10), an arbitrary scalar function $G$ of fields and antifields transforms as $G \rightarrow G+\delta G$ where

$$
\delta G=\varepsilon(G, F)+O\left(\varepsilon^{2}\right) .
$$

An alternative approach [263, 27] uses a function which is a combination of original and transformed fields, $F_{2}\left[\Phi, \tilde{\Phi}^{*}\right]$, to produce a canonical transformation via

$$
\tilde{\Phi}^{A}=\frac{\partial_{l} F_{2}\left[\Phi, \tilde{\Phi}^{*}\right]}{\partial \tilde{\Phi}_{A}^{*}}, \quad \Phi_{A}^{*}=\frac{\partial_{r} F_{2}\left[\Phi, \tilde{\Phi}^{*}\right]}{\partial \Phi^{A}},
$$

where $\epsilon\left(F_{2}\right)=1$ and gh $\left[F_{2}\right]=-1$. Of course, one must require this change of variables to be non-singular. The advantage of using $F_{2}$ is that the antibracket structure is exactly preserved

$$
\left(\tilde{\Phi}^{A}, \tilde{\Phi}_{B}^{*}\right)=\delta_{B}^{A} \quad, \quad\left(\tilde{\Phi}^{A}, \tilde{\Phi}^{B}\right)=\left(\tilde{\Phi}_{A}^{*}, \tilde{\Phi}_{B}^{*}\right)=0 .
$$

so that $F_{2}$ generates a finite transformation. A proof of Eq.(4.14) is given in ref.[251]. In using Eq.4.13), one must solve for $\Phi^{A}$ and $\Phi_{A}^{*}$ in terms of $\tilde{\Phi}^{A}$ and $\tilde{\Phi}_{A}^{*}$, i. e., $\Phi^{A}=\bar{\Phi}^{A}\left[\tilde{\Phi}, \tilde{\Phi}^{*}\right]$ and $\Phi_{A}^{*}=\bar{\Phi}_{A}^{*}\left[\tilde{\Phi}, \tilde{\Phi}^{*}\right]$. Then the canonical transformation corresponds to the shifts $\Phi^{A} \rightarrow \bar{\Phi}^{A}\left[\Phi, \Phi^{*}\right]$ and $\Phi_{A}^{*} \rightarrow \bar{\Phi}_{A}^{*}\left[\Phi, \Phi^{*}\right]$. Infinitesimal transformations are recovered using

$$
F_{2}=\tilde{\Phi}_{A}^{*} \Phi^{A}+\varepsilon f_{2}\left[\Phi, \tilde{\Phi}^{*}\right] .
$$

When $f_{2}=-F$, Eq.(4.10) is reproduced to order $\varepsilon$.

The role of the antibracket at quantum level is discussed in Sect. 8 .

\subsection{Classical Master Equation and Boundary Conditions}

Let $S\left[\Phi, \Phi^{*}\right]$ be an arbitrary functional of fields and antifields with the dimensions of action and with ghost number zero and even statistics: $\epsilon(S)=0$ and $\operatorname{gh}[S]=0$. The equation

$$
(S, S)=2 \frac{\partial_{r} S}{\partial \Phi^{A}} \frac{\partial_{l} S}{\partial \Phi_{A}^{*}}=0
$$


is called the classical master equation. This simple looking equation is the main topic of this subsection.

Not every solution to Eq.(4.16) is of interest. It is necessary to satisfy certain boundary conditions. A relevant solution plays a double role. On one hand, a solution $S$ is the generating functional for the structure functions of the gauge algebra. All relations among structure functions are contained in Eq.(1.16), thereby reproducing the equations in Sect. 2.4 and generalizing them to the generic $L$-th stage reducible theory. On the other hand, $S$ is the starting action to quantize covariantly the theory. After a gauge-fixing procedure is implemented, one can commence perturbation theory. The latter aspects are treated in Sect. 6.

Regard $S$ as the action for fields and antifields. The variations of $S$ with respect $\Phi^{A}$ and $\Phi_{A}^{*}$ are the equations of motion:

$$
\frac{\partial_{r} S}{\partial z^{a}}=0
$$

where the collective variables $z^{a}$ in Eq.(4.8) are used. We assume there exists a least one stationary point for which Eq.(4.17) holds. We let $\Sigma$ denote this subspace of stationary points in the full space of fields and antifields:

$$
\Sigma \longleftrightarrow\left\{\frac{\partial_{r} S}{\partial z^{a}}=0\right\}
$$

It turns out that, given a classical solution $\phi_{0}$ of $S_{0}$ as in Eq.(2.9), one possible stationary point is

$$
\phi^{j}=\phi_{0}^{j}, \quad \mathcal{C}_{s}^{\alpha_{s}}=0 ; \quad s=0, \ldots, L ; \quad \alpha_{s}=1, \ldots, m_{s}, \quad \Phi_{A}^{*}=0 .
$$

An action $S$, satisfying the master equation (4.16), possesses its own set of gauge invariances. Indeed, by differentiating Eq.(4.16) with respect to $z^{b}$, one finds after a little algebra that

$$
\frac{\partial_{r} S}{\partial z^{a}} \mathcal{R}_{b}^{a}=0, \quad a=1, \ldots, 2 N
$$

where

$$
\mathcal{R}_{b}^{a} \equiv \zeta^{a c} \frac{\partial_{l} \partial_{r} S}{\partial z^{c} \partial z^{b}}
$$

Although there appears to be $2 N$ gauge invariances, not all of them are independent on-shell as we now demonstrate. Differentiating Eq.(4.20) with respect to $z^{d}$, multiplying by $\zeta^{c d}$, using the definition of $\mathcal{R}_{b}^{a}$ in Eq.4.21) and imposing the stationary condition in Eq.(4.17), one finds that

$$
\left.\mathcal{R}_{a}^{c} \mathcal{R}_{b}^{a}\right|_{\Sigma}=0
$$


where $\Sigma$ denotes the space satisfying the on-shell condition in Eq.(4.18). One concludes that $\mathcal{R}_{b}^{a}$ is on-shell nilpotent. It is an elementary result of matrix theory that a nilpotent $2 N \times 2 N$ matrix has rank less than or equal to $N$. Hence, at the stationary point there exist at least $N$ relations among the gauge generators $\mathcal{R}_{b}^{a}$ and therefore the number $2 N-r$ of independent gauge transformations on-shell is greater than or equal to $N$, where $r$ is the rank of the hessian of $S$ at the stationary point:

$$
\left.r \equiv \operatorname{rank} \frac{\partial_{l} \partial_{r} S}{\partial z^{a} \partial z^{b}}\right|_{\Sigma}
$$

Necessarily,

$$
r \leq N
$$

A solution to the master equation is called proper if

$$
r=N,
$$

where $r$ is given in Eq.(4.22). When $r<N$, there are solutions to $\frac{\partial_{r} S}{\partial z^{a}} \delta z^{a}=0$ other than the ones given by Eqs.(4.20) and (4.21).

Usually, only proper solutions are of interest. The reason is simple. The action $S$ contains the physical fields $\phi^{i}$ and the ghost fields necessary for quantization. However, the antifields $\Phi_{A}^{*}, A=1, \ldots, N$, are unphysical. If $r=N$, then the number of independent gauge invariances of the type in Eq.(4.20) is the number of antifields. As a consequence, one can remove the $N$ non-physical variables $\Phi_{A}^{*}$, while maintaining the $N$ fields $\Phi^{A}$. At a later stage, the antifields can be eliminated through a gaugefixing procedure. Throughout the rest of this article, we restrict the discussion to the proper case.

We have not yet specified the relation between $S_{0}$ and $S$. To make contact with the original theory, one requires the proper solution to contain the original action $S_{0}[\phi]$. This requirement ensures the correct classical limit. It corresponds to the following boundary condition on $S$

$$
\left.S\left[\Phi, \Phi^{*}\right]\right|_{\Phi^{*}=0}=S_{0}[\phi]
$$

An additional boundary requirement is

$$
\left.\frac{\partial_{l} \partial_{r} S}{\partial \mathcal{C}_{s-1, \alpha_{s-1}}^{*} \partial \mathcal{C}_{s}^{\alpha_{s}}}\right|_{\Phi^{*}=0}=R_{s \alpha_{s}}^{\alpha_{s-1}}(\phi), \quad s=0, \ldots, L
$$

where $\mathcal{C}_{s-1, \alpha_{s-1}}^{*}$ is the antifield of $\mathcal{C}_{s-1}^{\alpha_{s-1}}$ :

$$
\mathcal{C}_{s, \alpha_{s}}^{*} \equiv\left(\mathcal{C}_{s}^{\alpha_{s}}\right)^{*}
$$


In Eq.(4.25), for notational convenience we define

$$
\mathcal{C}_{-1}^{\alpha_{-1}} \equiv \phi^{i}, \quad \mathcal{C}_{-1, \alpha_{-1}}^{*} \equiv \phi_{i}^{*}, \quad \text { with } \alpha_{-1}=i \quad .
$$

Actually, Eq.(4.25) does not need to be imposed as a separate boundary condition. Although it is not obvious, the requirement of being proper (4.23) and the classical boundary condition in Eq.(4.24) necessarily imply that a solution $S$ must satisfy Eq.(4.25) [28].

The proper solution $S$ can be expanded in a power series in antifields. Given the ghost number restriction on $S$ and the boundary conditions in Eqs.(4.24) and (4.25), the expansion necessarily begins as

$$
S\left[\Phi, \Phi^{*}\right]=S_{0}[\phi]+\sum_{s=0}^{L} \mathcal{C}_{s-1, \alpha_{s-1}}^{*} R_{s \alpha_{s}}^{\alpha_{s-1}} \mathcal{C}_{s}^{\alpha_{s}}+O\left(C^{* 2}\right) .
$$

In the next subsection we obtain the leading terms in the solution for a first-stage reducible theory. We also demonstrate how the gauge algebra is encoded in the classical master equation, when the classical basis is used.

\subsection{The Proper Solution and the Gauge Algebra}

This subsection establishes the connection between a proper solution to the classical master equation and the equations that the gauge-structure tensors must satisfy. For a first-stage reducible theory the minimum set of fields is $\phi^{i}, \mathcal{C}_{0}^{\alpha_{0}}$ (which we call $\mathcal{C}^{\alpha}$ ) and $\mathcal{C}_{1}^{\alpha_{1}}$ (which we call $\eta^{a}$ ). One expands the action $S\left[\Phi, \Phi^{*}\right]$ as a Taylor series in ghosts and antifields, while requiring the total ghost number to be zero. The result is

$$
\begin{aligned}
S\left[\Phi, \Phi^{*}\right]= & S_{0}[\phi]+\phi_{i}^{*} R_{\alpha}^{i} \mathcal{C}^{\alpha}+\mathcal{C}_{\alpha}^{*}\left(Z_{a}^{\alpha} \eta^{a}+\frac{1}{2} T_{\beta \gamma}^{\alpha}(-1)^{\epsilon_{\beta}} \mathcal{C}^{\gamma} \mathcal{C}^{\beta}\right) \\
& +\eta_{a}^{*}\left(A_{b \alpha}^{a} \eta^{b} \mathcal{C}^{\alpha}+\frac{1}{2} F_{\alpha \beta \gamma}^{a}(-1)^{\epsilon_{\beta}} \mathcal{C}^{\gamma} \mathcal{C}^{\beta} \mathcal{C}^{\alpha}\right) \\
& \phi_{i}^{*} \phi_{j}^{*}(-1)^{\epsilon_{i}}\left(\frac{1}{2} V_{a}^{j i} \eta^{a}+\frac{1}{4} E_{\alpha \beta}^{j i}(-1)^{\epsilon_{\alpha}} \mathcal{C}^{\beta} \mathcal{C}^{\alpha}\right) \\
+ & \mathcal{C}_{\delta}^{*} \phi_{i}^{*}(-1)^{\epsilon_{\delta}}\left(G_{a \beta}^{i \delta} \eta^{a} \mathcal{C}^{\beta}-\frac{1}{2} D_{\alpha \beta \gamma}^{i \delta}(-1)^{\epsilon_{\beta}} \mathcal{C}^{\gamma} \mathcal{C}^{\beta} \mathcal{C}^{\alpha}\right) \\
+ & \frac{1}{12} \phi_{i}^{*} \phi_{j}^{*} \phi_{k}^{*}(-1)^{\epsilon_{j}} M_{\alpha \beta \gamma}^{k j i}(-1)^{\epsilon_{\beta}} \mathcal{C}^{\gamma} \mathcal{C}^{\beta} \mathcal{C}^{\alpha}+\ldots,
\end{aligned}
$$

where, with the exception of $R_{\alpha}^{i}$ and $Z_{a}^{\alpha}$, which are fixed by the boundary condition in Eq.(4.28), one should, at this stage, think of the tensors, $T_{\alpha \beta}^{\gamma}$, $E_{\alpha \beta}^{j i}$, etc. in Eq.4.29) as having no relation to the tensors found in the gauge algebra structure of Sect. 2.4. The minus signs and fractions, $\frac{1}{2}, \frac{1}{4}$, and $\frac{1}{12}$, in Eq.(4.29) are put in for convenience. 
The goal is to find a solution to Eq.(4.16). It is necessary to compute derivatives of $S$ with respect to fields and antifields. One finds

$$
\begin{aligned}
& \frac{\partial_{r} S}{\partial \phi^{i}}=S_{0, i}+\phi_{j}^{*}\left(R_{\alpha}^{j} \mathcal{C}^{\alpha}\right)_{, i}+\mathcal{C}_{\alpha}^{*}\left(Z_{a}^{\alpha} \eta^{a}+\frac{1}{2} T_{\beta \gamma}^{\alpha}(-1)^{\epsilon_{\beta}} \mathcal{C}^{\gamma} \mathcal{C}^{\beta}\right)_{, i}+ \\
& \frac{1}{4} \phi_{j}^{*} \phi_{k}^{*}(-1)^{\epsilon_{j}}\left(E_{\alpha \beta}^{k j}(-1)^{\epsilon_{\alpha}} \mathcal{C}^{\beta} \mathcal{C}^{\alpha}\right)_{, i}+\ldots \\
& \frac{\partial_{l} S}{\partial \phi_{i}^{*}}=R_{\alpha}^{i} \mathcal{C}^{\alpha}+\phi_{j}^{*}(-1)^{\epsilon_{i}}\left(V_{a}^{j i} \eta^{a}+\frac{1}{2} E_{\alpha \beta}^{j i}(-1)^{\epsilon_{\alpha}} \mathcal{C}^{\beta} \mathcal{C}^{\alpha}\right) \\
& +(-1)^{\epsilon_{\delta} \epsilon_{i}} \mathcal{C}_{\delta}^{*}\left(G_{a \beta}^{i \delta} \eta^{a} \mathcal{C}^{\beta}-\frac{1}{2} D_{\alpha \beta \gamma}^{i \delta}(-1)^{\epsilon_{\beta}} \mathcal{C}^{\gamma} \mathcal{C}^{\beta} \mathcal{C}^{\alpha}\right)+ \\
& +\frac{1}{4}(-1)^{\epsilon_{j}} \phi_{j}^{*} \phi_{k}^{*} M_{\alpha \beta \gamma}^{k j i}(-1)^{\epsilon_{\beta}} \mathcal{C}^{\gamma} \mathcal{C}^{\beta} \mathcal{C}^{\alpha}+\ldots, \\
& \frac{\partial_{r} S}{\partial \mathcal{C}^{\alpha}}=\phi_{i}^{*} R_{\alpha}^{i}+\mathcal{C}_{\gamma}^{*} T_{\alpha \beta}^{\gamma}(-1)^{\epsilon_{\alpha}} \mathcal{C}^{\beta}+(-1)^{\epsilon_{i}} \frac{1}{2} \phi_{i}^{*} \phi_{j}^{*} E_{\alpha \beta}^{j i}(-1)^{\epsilon_{\alpha}} \mathcal{C}^{\beta}+\ldots, \\
& \frac{\partial_{l} S}{\partial \mathcal{C}_{\alpha}^{*}}=Z_{a}^{\alpha} \eta^{a}+\frac{1}{2} T_{\beta \gamma}^{\alpha}(-1)^{\epsilon_{\beta}} \mathcal{C}^{\gamma} \mathcal{C}^{\beta}-\frac{1}{2}(-1)^{\epsilon_{\alpha}} \phi_{i}^{*} D_{\beta \gamma \delta}^{i \alpha}(-1)^{\epsilon_{\gamma}} \mathcal{C}^{\delta} \mathcal{C}^{\gamma} \mathcal{C}^{\beta}+\ldots \\
& \frac{\partial_{r} S}{\partial \eta^{a}}=\mathcal{C}_{\alpha}^{*} Z_{a}^{\alpha}+\phi_{i}^{*} \phi_{j}^{*}(-1)^{\epsilon_{i}} \frac{1}{2} V_{a}^{j i}+\ldots, \\
& \frac{\partial_{l} S}{\partial \eta_{a}^{*}}=A_{b \alpha}^{a} \eta^{b} \mathcal{C}^{\alpha}+\frac{1}{2} F_{\alpha \beta \gamma}^{a}(-1)^{\epsilon_{\beta}} \mathcal{C}^{\gamma} \mathcal{C}^{\beta} \mathcal{C}^{\alpha}+\ldots
\end{aligned}
$$

Although ghosts do not depend on $\phi^{i}$, we write ()$_{, i}$ in the first equation to avoid some minus signs.

Given the above equations, it is straightforward to form the sum over products of derivatives in Eq.(4.16). We leave this step to the reader and simple quote the result [24, 26]: The classical master equation (4.16) is satisfied if the tensors, $T_{\alpha \beta}^{\gamma}, E_{\alpha \beta}^{j i}$, etc. in Eq.(4.29) are the ones in Sect. 2.4. In other words, Eq.4.29) with the tensors identified as the ones in Sect. 2.4 is a proper solution to the master equation. One finds that $(S, S)=0$ implies the gauge structure in equations Eqs.(2.33), (2.34) and (2.39) - (2.42). The reason why one equation $(S, S)=0$ is able to reproduce many equations is that the coefficients of each ghost and antifield term must separately be zero. Up to overall factors, Eq.(2.33) is the term independent of antifields, Eq. (2.34) is the coefficient of $\phi_{i}^{*}$ which is bilinear in the $\mathcal{C}^{\alpha}$, Eq. (2.39) is the coefficient linear in $\mathcal{C}_{\delta}^{*}$ and trilinear in the $\mathcal{C}^{\alpha}$, Eq.(2.40) is the coefficient of $\phi_{i}^{*} \phi_{j}^{*}$ which is trilinear in the $\mathcal{C}^{\alpha}$, Eq.(2.41) is the coefficient linear in $\phi_{i}^{*}$ and in $\eta^{a}$, and Eq.(2.42) is the coefficient of $\mathcal{C}_{\gamma}^{*}$ which is linear in both $\eta^{a}$ and $\mathcal{C}^{\beta}$. The coefficients of higher-order terms produce the higher-order gauge structure equations. For an irreducible system, the proper solution is the above with $\eta^{a}$ and $\eta_{a}^{*}$ set to zero. 
Summarizing, the antibracket formalism using fields and antifields allows a simple determination of the relevant gauge structure tensors. The proper solution to the classical master equation is a compact way of expressing the relations among the structure tensors.

\subsection{Existence and Uniqueness}

At this point, there are two obvious and interesting questions. Does there always exist a proper solution to the classical master equation and is the proper solution unique? Given reasonable conditions, which include the regularity postulates of Appendix B, there always exists a proper solution. This result was obtained in refs. 263, 28] for the case of an irreducible theory. For a general $L$-th stage reducible theory, the theorem was proven in ref. 1105.

To gain insight into the question of uniqueness, assume that a proper solution $S$ has been found so that $(S, S)=0$. Suppose that one performs a canonical transformation via Eq.(4.13). Then the transformed $S$ also obeys the classical master equation because such canonical transformations preserve the antibracket. For an infinitesimal canonical transformation, the transformed proper solution $S^{\prime}$ is, according to Eq.(1.12), given by $S^{\prime}=S+\varepsilon(S, F)+O\left(\varepsilon^{2}\right)$. This is the only ambiguity in the proper solution. Given the minimal set of fields in Eq.(4.1), the proper solution to the classical master equation is unique up to canonical transformations. This result was obtained in refs. 263, 28, 105. Since our aim is pedagogical, we do not present the proof here. The interested reader can consult the above references. Below we illustrate the non-uniqueness question using the example of the spinless relativistic particle. Canonical transformations which lead to field redefinitions of ghosts correspond to the freedom of redefining the gauge generators. This leads to changes in the structure tensors of Sect. 2 and corresponds to the non-uniqueness mentioned at the end of Sect. 2.1.

When gauge-fixing and path integral quantization is considered, it is necessary to enlarge the minimal set of fields in Eq.(4.1) to a non-minimal set. It turns out that trivial variable pairs can be added to the theory while maintaining the classical master equation and its properness. Let $\Lambda$ and $\Pi$ be two new fields and let $\Lambda^{*}$ and $\Pi^{*}$ be the corresponding antifields. Choose the ghost numbers and statistics so that

$$
\begin{gathered}
\operatorname{gh}[\Pi]=\operatorname{gh}[\Lambda]+1, \\
\epsilon(\Pi)=\epsilon(\Lambda)+1(\bmod 2) .
\end{gathered}
$$

Then $\operatorname{gh}\left[\Lambda^{*}\right]=-\operatorname{gh}[\Lambda]-1=-\operatorname{gh}[\Pi]$ and $\epsilon\left(\Lambda^{*}\right)=\epsilon(\Lambda)+1(\bmod 2)=\epsilon(\Pi)$, so that

$$
S_{\text {trivial }}=\int \mathrm{d} x \Lambda^{*} \Pi
$$


can be added to $S$. Adding such a bilinear to $S$ does not ruin the classical master equation $(S, S)=0$. When a non-minimal set of fields is employed, the proper solution to the master equation is unique up to canonical transformations and the addition of trivial pairs.

\subsection{The Classical BRST Symmetry}

An important concept in gauge theories is BRST symmetry. The BRST symmetry is what remains of gauge invariance after gauge-fixing has been implemented. In this sense, it can be regarded as a substitute for gauge invariance. There are three important features governing the BRST transformation: nilpotency, graded derivation, and invariance of the action $S$.

Even before gauge-fixing, the field-antifield formalism has BRST symmetry. Via the antibracket, the generator $\delta_{B}$ of this symmetry is the proper solution $S$ itself. Define the classical BRST transformation of a functional $X$ of fields and antifields by

$$
\delta_{B} X \equiv(X, S) \quad .
$$

The transformation rule for fields and antifields is therefore

$$
\delta_{B} \Phi^{A}=\frac{\partial_{l} S}{\partial \Phi_{A}^{*}}, \quad \delta_{B} \Phi_{A}^{*}=-\frac{\partial_{l} S}{\partial \Phi^{A}}=(-1)^{\epsilon_{A}+1} \frac{\partial_{r} S}{\partial \Phi^{A}} .
$$

Note that the field-antifield action $S$ is classically BRST symmetric

$$
\delta_{B} S=0
$$

as a consequence of $(S, S)=0$.

The BRST operator $\delta_{B}$ is a nilpotent graded derivationf: Given two functionals $X$ and $Y$,

$$
\delta_{B}(X Y)=X \delta_{B} Y+(-1)^{\epsilon_{Y}}\left(\delta_{B} X\right) Y \quad,
$$

and

$$
\delta_{B}^{2} X=0
$$

\footnotetext{
${ }^{4}$ An alternative definition for $\delta_{B}$ given by

$$
\delta_{B} X \equiv(-1)^{\epsilon_{X}}(X, S)
$$
}

is used by some authors. In this case, the $\delta_{B}$ continues to be a nilpotent graded derivation, but acts from the left to right, i.e., 4.35) is replaced by

$$
\delta_{B}(X Y)=\left(\delta_{B} X\right) Y+(-1)^{\epsilon_{X}} X \delta_{B} Y .
$$


The nilpotency follows from two properties of the antibracket: the graded Jacobi identity and graded antisymmetry (see Eq.(4.5)). These properties imply $((X, S), S)=$ $-((S, S), X)+(-1)^{\epsilon_{X}+1}((S, X), S)=-((S, S), X)-((X, S), S)$ which leads to $((X, S), S)=-\frac{1}{2}((S, S), X)=0$. Therefore, $\delta_{B}^{2} X=((X, S), S)=0$.

A functional $\mathcal{O}$ is a classical observable if $\delta_{B} \mathcal{O}=0$ and $\mathcal{O} \neq \delta_{B} Y$ for some $Y$. Two observables are considered equivalent if they differ by a BRST variation [103, 105]. A linear combination of observables is an observable. Because of the graded derivation property of $\delta_{B}$ in Eq.(4.35), the product of two classical observables is BRST invariant. Thus, observables form an algebra.

For a closed irreducible theory, the BRST transformation rules for $\Phi^{A}$ and $\Phi_{A}^{*}$, which depend on the original action $S_{0}$ and the structure tensors $R$ and $T$, can be obtained using Eqs.(4.29) and (4.33):

$$
\begin{gathered}
\delta_{B} \phi^{i}=R_{\alpha}^{i} \mathcal{C}^{\alpha}+\ldots \\
\delta_{B} \mathcal{C}^{\alpha}=\frac{1}{2} T_{\beta \gamma}^{\alpha}(-1)^{\epsilon_{\beta}} \mathcal{C}^{\gamma} \mathcal{C}^{\beta}+\ldots \\
\delta_{B} \phi_{i}^{*}=-(-1)^{\epsilon_{i}} S_{0, i}-(-1)^{\epsilon_{\alpha} \epsilon_{i}} \phi_{j}^{*} R_{\alpha, i}^{j} \mathcal{C}^{\alpha}-\frac{1}{2}(-1)^{\left(\epsilon_{\alpha}+\epsilon_{\beta}+1\right) \epsilon_{i}+\epsilon_{\alpha}} \mathcal{C}_{\gamma}^{*} T_{\alpha \beta, i}^{\gamma} \mathcal{C}^{\beta} \mathcal{C}^{\alpha}+\ldots \\
\delta_{B} \mathcal{C}_{\alpha}^{*}=(-1)^{\epsilon_{\alpha}} \phi_{i}^{*} R_{\alpha}^{i}+\mathcal{C}_{\gamma}^{*} T_{\alpha \beta}^{\gamma} \mathcal{C}^{\beta}+\ldots
\end{gathered}
$$

where we have displayed the terms involving $S_{0}, R$ and $T$. When the gauge-structure equations hold off-shell at all levels, then the tensors $D_{\alpha \beta \gamma}^{i \delta}, M_{\alpha \beta \gamma}^{k j i}$, etc. in Eq.(4.29) are zero, and the proper action is linear in antifields. In this case, there are no additional terms in Eq.(4.37) for irreducible systems. We illustrate BRST symmetry in a few of the examples in the next section.

Because Sect. 1 has introduced many ideas, it is worth enumerating the most important ones. After reading this section, one should know the following tools and concepts: antifields and the antibracket, canonical transformations, the classical master equation, properness and proper solution, classical BRST symmetry, and classical observables. 


\section{Examples of Proper Solutions}

In this section, we present proper solutions to the master equation for the examples considered in Sect. 3. Given the gauge-structure tensors, Eq.(4.29) immediately provides the proper solution $S$ for an irreducible or first-stage-reducible theory. Since all but two of the example field theories fall into one of these two cases, the construction of $S$ is straightforward. For the abelian $p$-form theory, Eq.4.28) is needed. The infinitely reducible open bosonic string requires some guesswork to obtain $S$. Given $S$, the BRST transformations $\delta_{B}$ can be determined from Eq.(4.32). For reasons of space, we display these transformations only for the spinless relativistic particle, Yang-Mills theory and the open bosonic string field theory. In Sect. 5.1, it is shown how two proper solutions to the spinless relativistic particle are related by a canonical transformation.

For exercises, we suggest the following (i) verify $(S, S)=0$, (ii) determine the action of $\delta_{B}$ on the fields and antifields in each example, (iii) use the results of (ii) to verify that $S$ is invariant under BRST transformations. Since (iii) and (i) are related, the calculations are similar and the reader may want to do only one or the other.

\subsection{The Spinless Relativistic Particle}

The spinless relativistic particle, considered in Sect. 3.1, is an example of a closed irreducible theory. The minimal set of fields and antifields are

$$
\Phi^{A}=\left\{x^{\mu}, e, \mathcal{C}\right\}, \quad \Phi_{A}^{*}=\left\{x_{\mu}^{*}, e^{*}, \mathcal{C}^{*}\right\} \quad
$$

with statistical parities

$$
\epsilon\left(x^{\mu}\right)=\epsilon(e)=\epsilon\left(\mathcal{C}^{*}\right)=0, \quad \epsilon\left(x_{\mu}^{*}\right)=\epsilon\left(e^{*}\right)=\epsilon(\mathcal{C})=1
$$

and ghost numbers

$$
\operatorname{gh}\left[x^{\mu}\right]=\operatorname{gh}[e]=0, \quad \operatorname{gh}[\mathcal{C}]=1, \quad \operatorname{gh}\left[x_{\mu}^{*}\right]=\operatorname{gh}\left[e^{*}\right]=-1, \quad \operatorname{gh}\left[\mathcal{C}^{*}\right]=-2 .
$$

Two versions of the gauge transformations were presented. In the first, given in Eq.(3.3), the only non-zero structure tensors are the gauge generators $R_{\alpha}^{i}$ given in Eq.(3.5). Using this and the general solution in Eq.(4.29), one finds

$$
S\left[\Phi, \Phi^{*}\right]=S_{0}\left[x^{\mu}, e\right]+\int \mathrm{d} \tau\left(x_{\mu}^{*} \dot{x}^{\mu} \frac{\mathcal{C}}{e}+e^{*} \dot{\mathcal{C}}\right)
$$

where $S_{0}\left[x^{\mu}, e\right]$ is given in Eq.(3.1). In the second version, given in Eq.(3.6), the gauge generators $R_{\alpha}^{i}$ and the commutator structure constant $T_{\beta \gamma}^{\alpha}$ are non-zero. Using 
Eq.(4.29), one obtains

$$
S\left[\Phi, \Phi^{*}\right]=S_{0}\left[x^{\mu}, e\right]+\int \mathrm{d} \tau\left(x_{\mu}^{*} \dot{x}^{\mu} \mathcal{C}+e^{*} e \dot{\mathcal{C}}+e^{*} \dot{e} \mathcal{C}+\mathcal{C}^{*} \dot{\mathcal{C}}\right)
$$

By the uniqueness theorem of the previous section, the solutions in Eqs.(5.4) and (5.5) must be related by a canonical transformation. In fact, Eq.(5.4) is mapped to Eq.(5.5) if $x^{\mu}, x_{\mu}^{*}$ and $e$ are unchanged but

$$
\mathcal{C} \rightarrow e \mathcal{C}, \quad \mathcal{C}^{*} \rightarrow \frac{1}{e} \mathcal{C}^{*}, \quad e^{*} \rightarrow e^{*}-\frac{1}{e} \mathcal{C}^{*} \mathcal{C}
$$

One can check that this is a canonical transformation by verifying that the antibracket structure in Eq.(4.11) is preserved. The infinitesimal version of the transformation is generated by the fermion

$$
F=\ln (e) \mathcal{C}^{*} \mathcal{C},
$$

when $F$ is used in Eq.(4.10). Infinitesimally,

$$
\delta \mathcal{C}=\varepsilon \ln (e) \mathcal{C}, \quad \delta \mathcal{C}^{*}=-\varepsilon \ln (e) \mathcal{C}^{*}, \quad \delta e^{*}=-\varepsilon \frac{1}{e} \mathcal{C}^{*} \mathcal{C}
$$

The finite transformation is obtained by iterating the infinitesimal transformation $N$ times, requiring $N \varepsilon=1$ and then letting $N \rightarrow \infty$. Alternatively, the full transformation can be generated using

$$
F_{2}=\int \mathrm{d} \tau\left(\tilde{x}_{\mu}^{*} x^{\mu}+\tilde{e}^{*} e+\frac{\tilde{\mathcal{C}}^{*} \mathcal{C}}{e}\right)
$$

and Eq.(4.13).

It is straightforward to obtain the BRST transformation rules using Eq.(4.33). From Eq.(5.4), one finds

$$
\begin{gathered}
\delta_{B} e=\dot{\mathcal{C}}, \quad \delta_{B} x^{\mu}=\frac{\dot{x}^{\mu} \mathcal{C}}{e}, \quad \delta_{B} \mathcal{C}=0 \\
\delta_{B} e^{*}=\frac{1}{2}\left(\frac{\dot{x}^{2}}{e^{2}}+m^{2}\right)+\frac{x_{\mu}^{*} \dot{x}^{\mu} \mathcal{C}}{e^{2}}, \\
\delta_{B} x_{\mu}^{*}=\frac{d}{d \tau}\left(\frac{\dot{x}_{\mu}+x_{\mu}^{*} \mathcal{C}}{e}\right), \\
\delta_{B} \mathcal{C}^{*}=\frac{x_{\mu}^{*} \dot{x}^{\mu}}{e}-\dot{e}^{*} .
\end{gathered}
$$

It is a useful exercise to verify the nilpotency of $\delta_{B}$ when acting on any field or antifield. One must be careful of signs. In this regard Eq.4.35 is useful. 


\subsection{Yang-Mills Theories}

Yang-Mills theories have a closed irreducible algebra. The number of gauge parameters is the rank of the group. There is a ghost field $\mathcal{C}^{a}$ for each gauge parameter $\Lambda^{a}$ in Eq.(3.15). Hence the fields and antifields are

$$
\Phi^{A}=\left\{A^{a \mu}, \mathcal{C}^{a}\right\}, \quad \Phi_{A}^{*}=\left\{A_{a \mu}^{*}, \mathcal{C}_{a}^{*}\right\}
$$

From Eq.4.29), the proper solution is

$$
S=\int \mathrm{d}^{d} x\left\{-\frac{1}{4} F_{\mu \nu}^{a} F_{a}^{\mu \nu}+A_{a \mu}^{*} D^{\mu a} \mathcal{C}^{b}+\frac{1}{2} \mathcal{C}_{c}^{*} f_{a b}{ }^{c} \mathcal{C}^{b} \mathcal{C}^{a}\right\} .
$$

The first term is the classical action (3.12) and the second and third terms correspond respectively to the $R_{\alpha}^{i}$ and $T_{\alpha \beta}^{\gamma}$ terms in Eq.(4.29).

As a check, let us verify the classical master equation $(S, S)=0$. Using Eq.(4.16), one finds

$$
\begin{gathered}
(S, S)=\int \mathrm{d}^{d} x\left(D^{\nu} F_{\nu \mu}\right)_{a} D^{\mu a}{ }_{b} \mathcal{C}^{b} \\
-\int \mathrm{d}^{d} x A_{d \mu}^{*} f_{a c}{ }^{d} \mathcal{C}^{c} D^{\mu a}{ }_{b} \mathcal{C}^{b}+\frac{1}{2} \int \mathrm{d}^{d} x A_{d \mu}^{*} D^{\mu d}{ }_{c} f_{a b}{ }^{c} \mathcal{C}^{b} \mathcal{C}^{a} \\
+\frac{1}{4} \int \mathrm{d}^{d} x \mathcal{C}_{e}^{*} f_{d c}{ }^{e} \mathcal{C}^{c} f_{a b}{ }^{d} \mathcal{C}^{b} \mathcal{C}^{a}
\end{gathered}
$$

The first term vanishes when one integrates by parts, uses the antisymmetry of $F_{\mu \nu}$ in $\mu$ and $\nu$, employs Eq. (3.19) and makes use of the antisymmetry of $f_{a b}{ }^{c}$ in $a$ and $b$. The second and third terms cancel when one makes use of the anticommuting nature of $\mathcal{C}^{a}$ and $\mathcal{C}^{b}$, of the antisymmetry of $f_{a b}{ }^{c}$ in $a$ and $b$, and of the Jacobi identity for the Lie group structure constants in Eq.(3.11). The last term vanishes for similar reasons. Hence, $(S, S)=0$.

The BRST transformation rules are obtained from Eqs.(4.33) and (5.9):

$$
\begin{gathered}
\delta_{B} A^{a \mu}=D^{\mu a}{ }_{b} \mathcal{C}^{b} \\
\delta_{B} \mathcal{C}^{a}=\frac{1}{2} f_{b c}{ }^{a} \mathcal{C}^{c} \mathcal{C}^{b}, \\
\delta_{B} A_{a \mu}^{*}=-\left(D^{\nu} F_{\nu \mu}\right)_{a}+f_{a b}{ }^{c} A_{c \mu}^{*} \mathcal{C}^{b} \\
\delta_{B} \mathcal{C}_{a}^{*}=-\left(D^{\mu} A_{\mu}^{*}\right)_{a}+\mathcal{C}_{c}^{*} f_{a b}{ }^{c} \mathcal{C}^{b}
\end{gathered}
$$




\subsection{Topological Yang-Mills Theory}

This theory is first-stage reducible. The action given in Eq.(3.20) is invariant under two types of gauge transformations. The gauge parameters associated with these transformations are $\Lambda^{a}$ of Eq.(3.15) and $\varepsilon^{a \mu}$ of Eq.(3.21). Correspondingly, one needs to introduce ghosts $\mathcal{C}^{a} \leftrightarrow \Lambda^{a}$ and $\mathcal{C}^{a \mu} \leftrightarrow \varepsilon^{a \mu}$. For the gauge invariances of the

gauge invariances, the ghost-for-ghost field $\eta^{a}$ must be introduced. Hence the fields and antifield are

$$
\Phi^{A}=\left\{A^{a \mu}, \mathcal{C}^{a}, \mathcal{C}^{a \mu}, \eta^{a}\right\}, \quad \Phi_{A}^{*}=\left\{A_{a \mu}^{*}, \mathcal{C}_{a}^{*}, \mathcal{C}_{a \mu}^{*}, \eta_{a}^{*}\right\}
$$

The non-zero gauge tensors are the gauge generators $R_{\alpha}^{i}$ given in Eq.(3.23), the commutator structure constants $T_{\alpha \beta}^{\gamma}$ given in Eq.(3.25), the null vectors $Z_{a}^{\alpha}$ given in Eq.(3.24), and $A_{b \alpha}^{a}$ given in Eq.(3.26). Other tensors are zero. Inserting the non-zero tensors in Eq. (4.29), one finds [137]

$$
\begin{aligned}
S= & \int \mathrm{d}^{4} x\left\{\frac{1}{4} F_{\mu \nu}^{a}{ }^{*} F_{a}^{\mu \nu}+A_{a \mu}^{*}\left(D^{\mu a}{ }_{b} \mathcal{C}^{b}+\mathcal{C}^{a \mu}\right)+\frac{1}{2} \mathcal{C}_{c}^{*} f_{a b}{ }^{c} \mathcal{C}^{b} \mathcal{C}^{a}\right. \\
& \left.+\mathcal{C}_{c \mu}^{*} f_{a b}{ }^{c} \mathcal{C}^{b \mu} \mathcal{C}^{a}+\mathcal{C}_{b}^{*} \eta^{b}-\mathcal{C}_{a \mu}^{*} D^{\mu a}{ }_{b} \eta^{b}+\eta_{c}^{*} f_{b a}{ }^{c} \eta^{a} \mathcal{C}^{b}\right\} .
\end{aligned}
$$

\subsection{The Antisymmetric Tensor Field Theory}

This system has been treated by the antifield formalism in refs. [73, 29, 34, 30, 86, 103, 12. The theory is on-shell first-stage reducible. The fields and antifields are

$$
\Phi^{A}=\left\{A^{a \mu}, B^{a \mu \nu}, \mathcal{C}^{a \mu}, \eta^{a}\right\}, \quad \Phi_{A}^{*}=\left\{A_{a \mu}^{*}, B_{a \mu \nu}^{*}, \mathcal{C}_{a \mu}^{*}, \eta_{a}^{*}\right\} .
$$

The non-zero structure tensors $R_{\alpha}^{i}, Z_{a}^{\alpha}$ and $V_{a}^{i j}$ are given in Eqs. 3.31) - 3.33). The proper solution is

$$
S=S_{0}+\int \mathrm{d}^{4} x\left(\frac{1}{2} B_{a}^{* \kappa \lambda} \varepsilon_{\kappa \lambda \mu \nu} D_{b}^{\mu a} \mathcal{C}^{b \nu}+\mathcal{C}_{b \mu}^{*} D^{\mu b}{ }_{a} \eta^{a}-\frac{1}{8} B_{c \rho \sigma}^{*} B_{\mu \nu}^{* b} \varepsilon^{\mu \nu \rho \sigma} f_{a b}{ }^{c} \eta^{a}\right)
$$

where $S_{0}$ is given in Eq.(3.27). An effect of the on-shell reducibility is the appearance of terms quadratic in the antifields.

\subsection{Abelian $p$-Form Theories}

Recall that these are examples of $p-1$ stage off-shell reducible theories. Consequently there are $p$ different types of ghosts, $\mathcal{C}_{0}, \mathcal{C}_{1}, \ldots, \mathcal{C}_{p-1}$, where $\mathcal{C}_{s}$ is a $p-1-s$ form:

$$
\Phi^{A}=\left\{A, \mathcal{C}_{0}, \mathcal{C}_{1}, \ldots, \mathcal{C}_{p-1}\right\}, \quad \Phi_{A}^{*}=\left\{A^{*}, \mathcal{C}_{0}^{*}, \mathcal{C}_{1}^{*}, \ldots, \mathcal{C}_{p-1}^{*}\right\}
$$


The proper solution is

$$
S=\int\left\{-\frac{1}{2} F \wedge^{*} F+{ }^{*}\left(A^{*}\right) \wedge d \mathcal{C}_{0}+\sum_{i=1}^{p-1} *\left(\mathcal{C}_{i-1}^{*}\right) \wedge d \mathcal{C}_{i}\right\},
$$

where $\wedge$ is the wedge product and a "*" in front of a field or antifield indicates the dual star operation. In Eq.(5.16), some antifields have been redefined by a minus sign factor compared to the definitions in Sect. 2. As a consequence of integration by parts and $d d=0$, as well as the definitions of the Hodge star operation and the wedge product, one can check that $(S, S)=0$. Indeed,

$$
\begin{gathered}
\frac{\partial_{r} S}{\partial A_{\mu_{1} \mu_{2} \ldots \mu_{p}}} \frac{\partial_{l} S}{\partial A^{* \mu_{1} \mu_{2} \ldots \mu_{p}}} \propto \int\left({ }^{*} d^{*} F\right) \wedge{ }^{*} d \mathcal{C}_{0} \propto \int d\left({ }^{*} F \wedge d \mathcal{C}_{0}\right)=0, \\
\frac{\partial_{r} S}{\partial\left(\mathcal{C}_{i}\right)_{\mu_{1} \mu_{2} \ldots \mu_{p-i-1}}} \frac{\partial_{l} S}{\partial\left(\mathcal{C}_{i}\right)^{* \mu_{1} \mu_{2} \ldots \mu_{p-i-1}}} \propto \\
\int\left({ }^{*} d^{*}\left(\mathcal{C}_{i}^{*}\right)\right) \wedge^{*} d \mathcal{C}_{i+1} \propto \int d\left({ }^{*}\left(\mathcal{C}_{i}^{*}\right) \wedge d \mathcal{C}_{i+1}\right)=0, \quad i=0,1, \ldots, p-1,
\end{gathered}
$$

where $\mathcal{C}_{-1}^{*} \equiv A^{*}$.

\subsection{Open String Field Theory}

This is an infinite-stage reducible theory. Hence, there are ghosts, ghosts for ghosts, ghosts for ghosts for ghosts, etc.. The fields are the string field $A$ and the infinite tower $\mathcal{C}_{s}, s=0, \ldots, \infty$ :

$$
\Phi^{A}=\left\{A, \mathcal{C}_{0}, \mathcal{C}_{1}, \mathcal{C}_{2}, \ldots\right\}, \quad \Phi_{A}^{*}=\left\{A^{*}, \mathcal{C}_{0}^{*}, \mathcal{C}_{1}^{*}, \mathcal{C}_{2}^{*}, \ldots\right\}
$$

where $A$ is a string 1 -form, $\mathcal{C}_{0}$ is a string 0 -form, $\mathcal{C}_{1}$ is a string -1 -form, etc.. Likewise $A^{*}$ is a string 1 -form, $\mathcal{C}_{0}^{*}$ is a string 0 -form, $\mathcal{C}_{1}^{*}$ is a string -1-form, etc.. In ChernSimons string field theory, odd forms have odd grading and even forms have even grading. In addition, according to Eq.(4.2) of the field-antifield formalism, $\varepsilon\left(\mathcal{C}_{s}\right)$ is zero for $s$ odd and one for $s$ even. With respect to the calculus of string differential forms, the total statistics is important. Consequently, for any fields and/or antifields $\varphi^{i}$ and $\varphi^{j}$, axioms (3) and (5) of Sect. 3.6, namely the graded distributive property of $Q$ across the star product and the graded commutativity of the star product under the integral, become

$$
\begin{gathered}
Q\left(\varphi^{i} * \varphi^{j}\right)=Q \varphi^{i} * \varphi^{j}+(-1)^{s_{i}} \varphi^{i} * Q \varphi^{j}, \\
\int \varphi^{i} * \varphi^{j}=(-1)^{s_{i} s_{j}} \int \varphi^{j} * \varphi^{i},
\end{gathered}
$$


where $s_{i}$ is the total statistics of $\varphi^{i}$ :

$$
s_{i} \equiv s\left(\varphi^{i}\right)=g\left(\varphi^{i}\right)+\epsilon\left(\varphi^{i}\right)(\bmod 2) \quad .
$$

Here, $g\left(\varphi^{i}\right)$ is the ghost number of $\varphi^{i}$ which is the same as the order of the string form, and $\epsilon\left(\varphi^{i}\right)$ is given by Eq.(4.2).

The quantization of the bosonic string was carried out by C. Thorn 246] and by M. Bochicchio [56]. Since this subject has been reviewed in ref. [247], we keep the discussion brief. To gain some insight in finding the proper solution, let us compute the non-zero terms in Eq.(4.29). The classical action $S_{0}$ is given in Eq.(3.39). The $R_{\alpha}^{i}, Z_{a}^{\alpha}, V_{a}^{i j}$ and $T_{\alpha \beta}^{\gamma}$ terms in Eq.(4.29) are respectively

$$
\begin{gathered}
\int{ }^{*} A^{*} *\left(Q \mathcal{C}_{0}+A * \mathcal{C}_{0}+\mathcal{C}_{0} * A\right), \\
\int{ }^{*} \mathcal{C}_{0}^{*} *\left(Q \mathcal{C}_{1}+A * \mathcal{C}_{1}+\mathcal{C}_{1} * A\right), \\
\int{ }^{*} A^{*} *{ }^{*} A^{*} * \mathcal{C}_{1}, \\
\int{ }^{*} \mathcal{C}_{0}^{*} * \mathcal{C}_{0} * \mathcal{C}_{0}
\end{gathered}
$$

where ${ }^{*}$ before a field is the string analog of the dual star operation determined by the bilinear form $\int A * B$. It takes $p$-forms into $3-p$ forms. Note that the structure of the above terms is similar to the classical action $S_{0}$ evaluated using various fields and antifields. The sign of the term $\mathcal{C}_{0} * A$ in the first equation is opposite to that of Eq.(3.42) because $\mathcal{C}_{0}$ has odd statistics. The field-antifield statistics, ghost number and total statistics for the fields are

$$
\begin{gathered}
\epsilon\left(\mathcal{C}_{i}\right)=i+1(\bmod 2), \quad g\left(\mathcal{C}_{i}\right)=-i, \quad \operatorname{gh}\left[\mathcal{C}_{i}\right]=i+1, \quad s\left(\mathcal{C}_{i}\right)=1, \\
\epsilon\left({ }^{*} \mathcal{C}_{i}^{*}\right)=i(\bmod 2), \quad g\left({ }^{*} \mathcal{C}_{i}^{*}\right)=i+3, \quad \operatorname{gh}\left[{ }^{*} \mathcal{C}_{i}^{*}\right]=-i-2, \quad s\left({ }^{*} \mathcal{C}_{i}^{*}\right)=1,
\end{gathered}
$$

for $i \geq-1$, where we have defined

$$
\mathcal{C}_{-1} \equiv A, \quad \mathcal{C}_{-1}^{*} \equiv A^{*} .
$$

Note that the total statistics of $\mathcal{C}_{i}$ and the ${ }^{*} \mathcal{C}_{i}^{*}$ is odd, so that it makes sense to define a field which is the formal sum

$$
\begin{gathered}
\Psi \equiv \ldots+{ }^{*}{ }^{*} \mathcal{C}_{s}^{*}+\ldots+{ }^{*} \mathcal{C}_{0}^{*}+{ }^{*} A^{*}+\stackrel{1}{A}+\mathcal{C}_{0}+\ldots+\overline{\mathcal{C}}_{s}^{s}+\ldots \\
\Psi=\sum_{p=-\infty}^{\infty} \psi_{p}, \text { where } \psi_{-p} \equiv \mathcal{C}_{p}, \text { for } p \geq-1, \quad \psi_{p} \equiv{ }^{*} \mathcal{C}_{p-3}^{*}, \text { for } p \geq 2 .
\end{gathered}
$$


In the first equation, the order of the string form is denoted above the field. The total statistics of the field-antifield $\Psi$ is odd: $s(\Psi)=1$. The terms in Eq.(5.20) and the classical action are both contained in the following ansatz for the proper solution

$$
\begin{gathered}
S=\frac{1}{2} \int \Psi * Q \Psi+\frac{1}{3} \int \Psi * \Psi * \Psi= \\
\frac{1}{2} \sum_{p=-\infty}^{\infty} \int \psi_{2-p} * Q \psi_{p}+\frac{1}{3} \sum_{\substack{p=-\infty \\
q=-\infty}}^{\infty} \int \psi_{p} * \psi_{q} * \psi_{3-p-q} .
\end{gathered}
$$

By obtaining the $L$-th stage reducible proper solution, computing the non-zero structure tensors, one could derive Eq.(5.24). Instead let us check the ansatz. One simply needs to verify the classical master equation

$$
0 \stackrel{?}{=}(S, S) \propto \int(Q \Psi+\Psi * \Psi) *(Q \Psi+\Psi * \Psi)
$$

Several terms are produced. The first is

$$
\int Q \Psi * Q \Psi=\int Q(\Psi * Q \Psi)=0
$$

where axioms (1), (2) and (3) of Sect. 3.6 have been used. Note that

$$
\begin{gathered}
0=\int Q(\Psi * \Psi * \Psi)= \\
\int(Q \Psi * \Psi * \Psi)-\int(\Psi * Q \Psi * \Psi)+\int(\Psi * \Psi * Q \Psi)= \\
3 \int(Q \Psi * \Psi * \Psi)=3 \int(\Psi * \Psi * Q \Psi),
\end{gathered}
$$

where axioms (2)-(5) are used. This implies that $\int(Q \Psi * \Psi * \Psi)=\int(\Psi * \Psi * Q \Psi)=$ 0, leading to the vanishing of two of the terms in Eq.(5.25). The last term in Eq. (5.25) is zero when axioms (4) and (5) are combined to give

$$
\int(\Psi * \Psi) *(\Psi * \Psi)=\int(\Psi * \Psi * \Psi * \Psi)=-\int(\Psi * \Psi * \Psi * \Psi),
$$

where Eq. (5.18) is used in the last step. Since the master equation is satisfied and the suitable boundary conditions are correctly implemented, Eq.(5.24) is a proper solution. The classical action in Eq.(3.39) and the proper solution in Eq.(5.24), although structurally identical, differ in that the field entering the action is different. In $S_{0}$ it is the string one-form $A$, while in $S$ it is the tower $\Psi$, given in Eq.(5.23), which includes ghosts and antighosts as well as $A$.

The BRST transformation rules for the fields and antifields are

$$
\delta_{B} \Psi=Q \Psi+\Psi * \Psi
$$


The transformation rule for a particular component $\psi_{p}$ of $\Psi$ in Eq.(5.23) is obtained by selecting the $p$-form term of Eq.(5.26).

Let us verify the nilpotency of $\delta_{B}$ :

$$
\begin{gathered}
\delta_{B}^{2} \Psi=Q \delta_{B} \Psi+\Psi * \delta_{B} \Psi-\delta_{B} \Psi * \Psi= \\
Q^{2} \Psi+Q(\Psi * \Psi)+\Psi * Q \Psi+\Psi *(\Psi * \Psi)-Q \Psi * \Psi-(\Psi * \Psi) * \Psi=0 .
\end{gathered}
$$

The last equality holds due to the axioms of open string field theory: The first term is zero because of axiom (1), the nilpotency of $Q$; the second, third and fifth terms cancel because of axiom (3), the graded distributive property of $Q$; and the fourth and sixth terms cancel by axiom (4), the associativity of the star product.

\subsection{The Massless Relativistic Spinning Particle}

This system has a closed irreducible algebra but possesses local supersymmetry. It has been treated by the antifield formalism in ref. [135. To the original fields, $x^{\mu}, \psi^{\mu}, e$, and $\chi$, one adds the ghosts $\mathcal{C}$ and $\Gamma$ respectively for general coordinate transformations and for local supersymmetry transformations. The fields and antifields are

$$
\Phi^{A}=\left\{x^{\mu}, \psi^{\mu}, e, \chi, \mathcal{C}, \Gamma\right\}, \quad \Phi_{A}^{*}=\left\{x_{\mu}^{*}, \psi_{\mu}^{*}, e^{*}, \chi^{*}, \mathcal{C}^{*}, \Gamma^{*}\right\} \quad,
$$

where $x^{\mu}, e, \Gamma, \psi_{\mu}^{*}, \chi^{*}$ and $\mathcal{C}^{*}$ are commuting while $\psi^{\mu}, \chi, \mathcal{C}, \Gamma^{*}, x_{\mu}^{*}$ and $e^{*}$ are anticommuting.

The only non-zero terms in Eq.(14.29) are $S_{0}, \phi_{i}^{*} R_{\alpha}^{i} \mathcal{C}^{\alpha}$ and $\frac{1}{2} \mathcal{C}_{\gamma}^{*} T_{\alpha \beta}^{\gamma}(-1)^{\epsilon_{\alpha}} \mathcal{C}^{\beta} \mathcal{C}^{\alpha}$. These quantities are determined in Eqs.(3.66)-(3.69). The result is

$$
\begin{gathered}
S=S_{0}+\int \mathrm{d} \tau\left\{x_{\mu}^{*}\left(-i e \psi^{\mu} \Gamma+\dot{x}^{\mu} \mathcal{C}\right)+\psi_{\mu}^{*}\left(\left(\dot{x}^{\mu}-i \chi \psi^{\mu}\right) \Gamma+\dot{\psi}^{\mu} \mathcal{C}\right)+\right. \\
e^{*}(-2 i e \chi \Gamma+\dot{e} \mathcal{C}+e \dot{\mathcal{C}})+\chi^{*}(e \dot{\Gamma}+\dot{e} \Gamma+\chi \dot{\mathcal{C}}+\dot{\chi} \mathcal{C})+ \\
\left.\mathcal{C}^{*}(\dot{\mathcal{C}} \mathcal{C}+i e \Gamma \Gamma)+\Gamma^{*}(\mathcal{C} \dot{\Gamma}-\dot{\mathcal{C}} \Gamma+i \chi \Gamma \Gamma)\right\} .
\end{gathered}
$$

where $S_{0}$ is given in Eq.(3.66)

\subsection{The First-Quantized Bosonic String}

This system has a closed irreducible algebra. There are three types of gauge invariances: local translations, local Lorentz boosts and scaling transformations to which we respectively assign the ghosts $\mathcal{C}^{m}, \mathcal{C}^{a b}$ and $\mathcal{C}$ where $a, b$ and $m$ take on the 
values $\sigma$ and $\tau$ and $\mathcal{C}^{b a}=-\mathcal{C}^{a b}$. Combining these ghosts with the original fields $X^{\mu}$ and $e_{a}{ }^{m}$, one arrives at the following set of fields and antifields:

$$
\Phi^{A}=\left\{X^{\mu}, e_{a}^{m}, \mathcal{C}^{m}, \mathcal{C}^{a b}, \mathcal{C}\right\} \quad, \quad \Phi_{A}^{*}=\left\{X_{\mu}^{*}, e^{* a}{ }_{m}, \mathcal{C}_{m}^{*}, \mathcal{C}_{a b}^{*}, \mathcal{C}^{*}\right\} \quad,
$$

where $\mu$ ranges from 0 to $D-1=25$. The only non-zero terms in Eq.(4.29) are $S_{0}$, $\phi_{i}^{*} R_{\alpha}^{i} \mathcal{C}^{\alpha}$ and $\frac{1}{2} \mathcal{C}_{\gamma}^{*} T_{\alpha \beta}^{\gamma}(-1)^{\epsilon_{\alpha}} \mathcal{C}^{\beta} \mathcal{C}^{\alpha}$. These tensors are computed in Sect. 3.8. One thus obtains

$$
\begin{gathered}
S=S_{0}+\int \mathrm{d} \tau \int_{0}^{\pi} \mathrm{d} \sigma\left\{X_{\mu}^{*} \mathcal{C}^{m} \partial_{m} X^{\mu}+\right. \\
e^{* a}{ }_{m}\left(\mathcal{C}^{n} \partial_{n} e_{a}{ }^{m}-e_{a}{ }^{n} \partial_{n} \mathcal{C}^{m}+e_{a}{ }^{m} \mathcal{C}+e_{b}{ }^{m} \eta_{a c} \mathcal{C}^{c b}\right) \\
\left.-\mathcal{C}_{m}^{*} \mathcal{C}^{n} \partial_{n} \mathcal{C}^{m}+\mathcal{C}_{a b}^{*}\left(\mathcal{C}^{a c} \mathcal{C}^{b d} \eta_{c d}-\mathcal{C}^{n} \partial_{n} \mathcal{C}^{a b}\right)-\mathcal{C}^{*} \mathcal{C}^{n} \partial_{n} \mathcal{C}\right\}
\end{gathered}
$$

where $S_{0}$ is given in Eq.(3.79). 


\section{The Gauge-Fixing Fermion}

This section discusses the process of gauge-fixing in the field-antifield formalism [24, 26], thereby paving the way to the quantization of gauge theories via the path integral approach. The important concept is a gauge-fixing fermion $\Psi$. It is a Grassmann-odd functional with ghost number -1. In Sect. 6.1, it is shown how antifields can be eliminated to obtain an action suitable for the computation of correlation functions in standard perturbation theory. Results are independent of $\Psi$ if the action satisfies the quantum master equation in Eq. (6.12] [23, 24]. To construct an appropriate $\Psi$, additional fields and their antifields are needed. See Sect. 6.2. Details of the gauge-fixing procedure for a general theory are presented in Sects. 6.3 and 6.4. Since Sects. 6.2 6.4 are somewhat technical, the reader may wish to read only the irreducible case, which is discussed at the beginning of each subsection. In particular instances, gauge-fixing can be done without $\Psi$ by performing a cleverly chosen canonical transformation [104, 230, 259, 44, 252, 257.

After gauge-fixing is completed, the theory is still invariant under gauge-fixed BRST transformations $\delta_{B_{\Psi}}$ [24]. The nilpotency of $\delta_{B_{\Psi}}$ is not guaranteed off-shell [255, 29, 30, 251]. However, when the equations of motion for the gauge-fixed action are used, $\delta_{B_{\Psi}}^{2}=0$. An attractive way of viewing the effects of gauge-fixing associated with $\Psi$ and the content of $\delta_{B_{\Psi}}$, is to perform a canonical transformation to the socalled gauge-fixed basis. In this basis, one retains antifields as sources of gauge-fixed BRST transformations, and the classical master equation reproduces the algebraic structure associated with $\delta_{B_{\Psi}}$.

\subsection{Generalities}

Although ghost fields have been incorporated into the theory, the field-antifield action $S$ still possesses gauge invariances (See Eq.(4.20)). Hence it is not yet suitable for quantization via the path integral approach. A gauge-fixing procedure is needed. The theory also contains many antifields that usually one wants to eliminate before computing amplitudes and $S$-matrix elements. One cannot simply set the antifields to zero because the action would reduce to the original classical action $S_{0}$, which is not appropriate for commencing perturbation theory due to gauge invariances. Following ref. [24], antifields are eliminated by using a gauge-fixing fermion $\Psi(\Phi)$ via

$$
\Phi_{A}^{*}=\frac{\partial \Psi}{\partial \Phi^{A}}
$$

Note that $\Psi$ is a functional of fields only. It does not matter whether right or left

derivatives are used in Eq. (6.1) because $\frac{\partial_{l} \Psi}{\partial \Phi^{A}}=\frac{\partial_{r} \Psi}{\partial \Phi^{A}}$ since $\epsilon(\Psi)=1$ (see Eq. (A.4) of 
Appendix A). We denote the "surface" in functional space determined by Eq.(6.1) by $\Sigma_{\Psi}$, 円

$$
\Sigma_{\Psi} \longleftrightarrow\left\{\Phi_{A}^{*}=\frac{\partial_{r} \Psi}{\partial \Phi^{A}}\right\}
$$

so that

$$
\left.X\right|_{\Sigma_{\Psi}} \equiv X\left[\Phi, \frac{\partial \Psi}{\partial \Phi}\right]
$$

The corresponding gauge-fixed action is $\left.S\right|_{\Sigma_{\Psi}} \equiv S_{\Psi}$. Matching the statistics and ghost numbers of $\Phi_{A}^{*}$ and $\frac{\partial \Psi}{\partial \Phi^{A}}$ leads to

$$
\epsilon(\Psi)=1 \quad, \quad \operatorname{gh}[\Psi]=-1 \quad .
$$

To quantize the theory, let us use the path integral approach with the constraint in Eq.(6.1) implemented by a delta function:

$$
I_{\Psi}(X)=\int[\mathrm{d} \Phi]\left[\mathrm{d} \Phi^{*}\right] \delta\left(\Phi_{A}^{*}-\frac{\partial \Psi}{\partial \Phi^{A}}\right) \exp \left(\frac{i}{\hbar} W\left[\Phi, \Phi^{*}\right]\right) X\left[\Phi, \Phi^{*}\right],
$$

where $X$ is a correlation function of interest. Here $W$ is the quantum generalization of the field-antifield action $S$. By the correspondence principle, the two should be equal in the $\hbar \rightarrow 0$ limit. One must choose $\Psi$ so that the theory is non-degenerate, that is, when the action is expanded about a solution of the equations of motion, propagators exist. Such a $\Psi$ is called admissible. Conditions on $\Psi$ assuring admissibility are given in Sect. 6.3 for certain gauge-fixing schemes. When $S_{0}$ is local, it is desirable that $\Psi$ be a local functional of the fields so as to preserve the locality of the gauge-fixed action. Selecting a "good" $\Psi$ is a matter of skill. A judicious choice can greatly simplify a particular computation, and the choice can depend on the type of computation, e.g. a correlation function, a proof of unitarity, a proof of renormalizability, etc..

The freedom in choosing $\Psi$ corresponds to the choice of the gauge-fixing procedure. One would like results to be independent of gauge fixing. Let us determine when this is the case. Define the integrand in Eq.(6.5) to be $\mathcal{I}$, i.e.,

$$
\mathcal{I}\left[\Phi, \Phi^{*}\right] \equiv \exp \left(\frac{i}{\hbar} W\left[\Phi, \Phi^{*}\right]\right) X\left[\Phi, \Phi^{*}\right]
$$

Under an infinitesimal change $\delta \Psi$ of $\Psi, I_{\Psi}(X)$ changes by

$$
I_{\Psi+\delta \Psi}(X)-I_{\Psi}(X)=\int[\mathrm{d} \Phi]\left(\mathcal{I}\left[\Phi, \frac{\partial \Psi}{\partial \Phi}+\frac{\partial \delta \Psi}{\partial \Phi}\right]-\mathcal{I}\left[\Phi, \frac{\partial \Psi}{\partial \Phi}\right]\right)
$$

\footnotetext{
${ }^{5}$ Do not confuse $\Sigma_{\Psi}$ with $\Sigma$ of Eq.(4.18). The latter $(\Sigma$ without a subscript $\Psi)$ corresponds to the on-shell condition.
} 


$$
\begin{aligned}
& =\int[\mathrm{d} \Phi] \frac{\partial_{r} \mathcal{I}}{\partial \Phi_{A}^{*}} \frac{\partial_{l} \delta \Psi}{\partial \Phi^{A}}+O\left((\delta \Psi)^{2}\right) \\
& =\int[\mathrm{d} \Phi] \Delta \mathcal{I} \delta \Psi+O\left((\delta \Psi)^{2}\right),
\end{aligned}
$$

where integration by parts (see Eq.(A.5) in Appendix A) has been used, and where the operator $\Delta$ is defined to be [24, 26]

$$
\Delta \equiv(-1)^{\epsilon_{A}+1} \frac{\partial_{r}}{\partial \Phi^{A}} \frac{\partial_{r}}{\partial \Phi_{A}^{*}} .
$$

It is a kind of "nilpotent divergence operator" in the space of fields and antifields. It formally satisfies

$$
\begin{gathered}
\Delta \Delta=0 \\
\Delta(X Y)=X \Delta Y+(-1)^{\epsilon_{Y}}(\Delta X) Y+(-1)^{\epsilon_{Y}}(X, Y), \\
\Delta((X, Y))=(X, \Delta Y)-(-1)^{\epsilon_{Y}}(\Delta X, Y) .
\end{gathered}
$$

Note that

$$
\operatorname{gh}[\Delta]=\epsilon(\Delta)=1 .
$$

According to the above calculation, the integral $I_{\Psi}(X)$ is infinitesimally independent of $\Psi$ if

$$
\Delta \mathcal{I}=0
$$

Eq.(6.10) is a requirement of a "good" integrand.

The path integral itself should be gauge independent. This corresponds to setting $X=1$ in $I_{\Psi}(X)$, from which one finds

$$
\Delta \exp \left(\frac{i}{\hbar} W\right)=\exp \left(\frac{i}{\hbar} W\right)\left(\frac{i}{\hbar} \Delta W-\frac{1}{2 \hbar^{2}}(W, W)\right)=0,
$$

so that one needs

$$
\frac{1}{2}(W, W)=i \hbar \Delta W .
$$

Eq.(6.12) is known as the quantum master equation [24, 26]. When $W$ satisfies Eq.(6.12), $X$ in Eq.(6.6) must satisfy

$$
(X, W)=i \hbar \Delta X
$$

to produce gauge-invariant correlation functions. Eqs. (6.12) and (6.13) summarize the conditions so that a computation does not depend on the choice of $\Psi$ [103, 152].

If $W$ is expanded in powers of $\hbar$ (the loop expansion) via

$$
W=S+\sum_{p=1}^{\infty} \hbar^{p} M_{p}
$$


the quantum master equation (Eq.(6.12)) implies that the first term $S$ and quantum correction terms $M_{p}$ must satisfy

$$
\begin{gathered}
(S, S)=0, \quad\left(M_{1}, S\right)=i \Delta S \quad, \\
\left(M_{n}, S\right)=i \Delta M_{n-1}-\frac{1}{2} \sum_{p=1}^{n-1}\left(M_{p}, M_{n-p}\right), \text { for } n \geq 2 .
\end{gathered}
$$

By the correspondence principle, $S$ in Eq.(6.14) is identified as the classical fieldantifield action and is consistent with Eqs.(6.12) and (6.15) because it satisfies the classical master equation $(S, S)=0$.

Likewise, when $X$ in Eq. (6.13) is expanded in an $\hbar$ series

$$
X=\sum_{p=0}^{\infty} \hbar^{p} X_{p},
$$

one finds that Eq.(6.13) implies

$$
\begin{gathered}
\left(X_{0}, S\right)=0 \\
\left(X_{n}, S\right)=i \Delta X_{n-1}-\sum_{p=1}^{n}\left(X_{n-p}, M_{p}\right), \text { for } n \geq 1 .
\end{gathered}
$$

The first equation says that the "classical" part of the quantum operator $X$ is classically BRST invariant.

\subsection{Gauge-Fixing Auxiliary Fields}

To eliminate antighost fields by Eq.(6.1), $\Psi$ must be a functional of fields and have ghost number -1 . However, the fields of the minimal sector introduced in Sect. 1 all have non-negative ghost numbers. Hence, it is impossible to construct an acceptable $\Psi$ unless one introduces additional fields. At this point, one takes advantage of adding trivial pairs (c.f. Sect. 4.5) to the theory. This subsection enumerates the auxiliary trivial pairs needed to obtain an admissible $\Psi$ [26, 119].

For an irreducible theory, one introduces one trivial pair

$$
\overline{\mathcal{C}}_{0 \alpha_{0}}, \quad \bar{\pi}_{0 \alpha_{0}},
$$

with statistics and ghost numbers equal to

$$
\begin{array}{cc}
\epsilon\left(\overline{\mathcal{C}}_{0 \alpha_{0}}\right)=\epsilon_{\alpha_{0}}+1, & \operatorname{gh}\left[\overline{\mathcal{C}}_{0 \alpha_{0}}\right]=-1, \\
\epsilon\left(\bar{\pi}_{0 \alpha_{0}}\right)=\epsilon_{\alpha_{0}}, \quad \operatorname{gh}\left[\bar{\pi}_{0 \alpha_{0}}\right]=0 .
\end{array}
$$


The fields $\overline{\mathcal{C}}_{0 \alpha_{0}}$ are the Faddeev-Popov antighosts of $\mathcal{C}_{0}^{\alpha_{0}}$.

For a first-stage reducible theory, one needs, in addition to the above pair, two more trivial pairs

$$
\begin{array}{lll}
\overline{\mathcal{C}}_{1 \alpha_{1}}, & \bar{\pi}_{1 \alpha_{1}}, \\
\mathcal{C}_{1}^{1 \alpha_{1}}, & \pi_{1}^{1 \alpha_{1}},
\end{array} .
$$

Their statistics and ghost numbers are

$$
\begin{gathered}
\epsilon\left(\overline{\mathcal{C}}_{1 \alpha_{1}}\right)=\epsilon\left(\mathcal{C}_{1}^{1 \alpha_{1}}\right)=\epsilon_{\alpha_{1}}, \\
\operatorname{gh}\left[\overline{\mathcal{C}}_{1 \alpha_{1}}\right]=-2, \quad \operatorname{gh}\left[\mathcal{C}_{1}^{1 \alpha_{1}}\right]=0, \\
\epsilon\left(\bar{\pi}_{1 \alpha_{1}}\right)=\epsilon\left(\pi_{1}^{1 \alpha_{1}}\right)=\epsilon_{\alpha_{1}}+1, \\
\operatorname{gh}\left[\bar{\pi}_{1 \alpha_{1}}\right]=-1, \quad \operatorname{gh}\left[\pi_{1}^{1 \alpha_{1}}\right]=1 .
\end{gathered}
$$

In general, for an $L$ th-stage reducible theory, for each integer $s$ ranging from 0 to $L$ one introduces $s+1$ trivial pairs [26]:

$$
\begin{gathered}
\overline{\mathcal{C}}_{s \alpha_{s}}^{k}, \bar{\pi}_{s \alpha_{s}}^{k}, \quad k=0,2,4, \ldots, 2\left[\frac{s}{2}\right], \\
\mathcal{C}_{s}^{k \alpha_{s}}, \pi_{s}^{k \alpha_{s}}, \quad k=1,3,5, \ldots, 2\left[\frac{s-1}{2}\right]+1,
\end{gathered}
$$

where $\alpha_{s}=1, \ldots, m_{s}$. The total number of auxiliary trivial pairs is $\sum_{s=0}^{L}(s+1)=$ $(L+1)(L+2) / 2$. The ghosts $\overline{\mathcal{C}}_{s \alpha_{s}}^{k}, \mathcal{C}_{s}^{k \alpha_{s}}$, in Eq.(6.22) for $k \geq 1$ are known as $e x$ traghosts, while the fields $\bar{\pi}_{s \alpha_{s}}^{k}, \pi_{s}^{k \alpha_{s}}$, are usually called Lagrange multipliers for reasons which become clear below. The original set of fields $\left\{\phi^{i}, C_{s}^{\alpha_{s}}\right\}$ of Sect. 国 is called the minimal set. The minimal sector fields together with the auxiliary fields in Eq.(6.22) are called the non-minimal set. In Eq.(6.22), [ ] stands for the greatest integer so that

$$
\left[\frac{m}{2}\right]= \begin{cases}\frac{m}{2}, & \text { if } m \text { is even } \\ \frac{m-1}{2}, & \text { if } m \text { is odd } .\end{cases}
$$

In general, the subscript $s$ on $\mathcal{C}_{s}^{k \alpha_{s}}$ and $\overline{\mathcal{C}}_{s \alpha_{s}}^{k}$ indicate the level of the field, whereas the superscript $k$ distinguishes different fields at the same level. It is convenient to associate $\alpha_{-1}$ with the index $i$ on $\phi^{i}$ and define

$$
\begin{gathered}
\mathcal{C}_{s}^{-1 \alpha_{s}} \equiv \mathcal{C}_{s}^{\alpha_{s}}, \quad \mathcal{C}_{-1}^{-1 \alpha_{-1}} \equiv \phi^{i}, \quad \alpha_{-1} \equiv i \\
\overline{\mathcal{C}}_{s \alpha_{s}}^{0} \equiv \overline{\mathcal{C}}_{s \alpha_{s}}, \quad \bar{\pi}_{s \alpha_{s}}^{0} \equiv \bar{\pi}_{s \alpha_{s}} .
\end{gathered}
$$

The following equations also apply to the minimal sector fields when $k=-1$ and 0 . 
The statistics of $\overline{\mathcal{C}}_{s \alpha_{s}}^{k}$ and $\mathcal{C}_{s}^{k \alpha_{s}}$ are the same as those of $\mathcal{C}_{s}^{\alpha_{s}}$, namely,

$$
\epsilon\left(\overline{\mathcal{C}}_{s \alpha_{s}}^{k}\right)=\epsilon\left(\mathcal{C}_{s}^{k \alpha_{s}}\right)=\epsilon_{\alpha_{s}}+s+1(\bmod 2),
$$

and their ghost numbers are

$$
\begin{gathered}
\operatorname{gh}\left[\overline{\mathcal{C}}_{s \alpha_{s}}^{k}\right]=k-s-1, \quad L \geq s \geq 0, \quad s \geq k \geq 0, \quad \text { for } k \text { even }, \\
\operatorname{gh}\left[\mathcal{C}_{s}^{k \alpha_{s}}\right]=s-k, \quad L \geq s \geq-1, \quad s \geq k \geq-1, \quad \text { for } k \text { odd } .
\end{gathered}
$$

The statistics and ghost numbers of $\bar{\pi}_{s \alpha_{s}}^{k}$ and $\pi_{s}^{k \alpha_{s}}$ are determined by the requirement that they form a trivial pair respectively with $\overline{\mathcal{C}}_{s \alpha_{s}}^{k}$ and $\mathcal{C}_{s}^{k \alpha_{s}}$. Using Eq. (4.30),

$$
\begin{gathered}
\epsilon\left(\bar{\pi}_{s \alpha_{s}}^{k}\right)=\epsilon\left(\pi_{s}^{k \alpha_{s}}\right)=\epsilon_{\alpha_{s}}+s(\bmod 2), \\
\operatorname{gh}\left[\bar{\pi}_{s \alpha_{s}}^{k}\right]=k-s, \quad L \geq s \geq 0, \quad s \geq k \geq 0, \quad \text { for } k \text { even }, \\
\operatorname{gh}\left[\pi_{s}^{k \alpha_{s}}\right]=s-k+1, \quad L \geq s \geq 1, \quad s \geq k \geq 1, \quad \text { for } k \text { odd } .
\end{gathered}
$$

The statistics and ghost numbers of the antifields are determined from Eq.(4.3).

The minimal sector fields along with the auxiliary $\overline{\mathcal{C}}_{s \alpha_{s}}^{k}$ and $\mathcal{C}_{s}^{k \alpha_{s}}$ can be arranged into the following useful triangular tableau [26]:

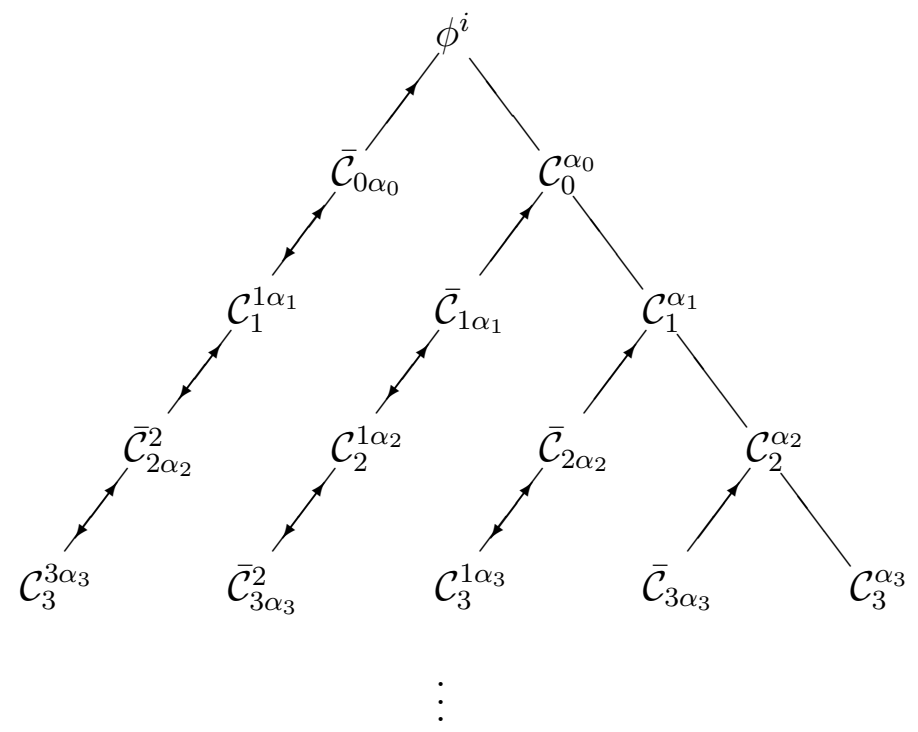

Diagram 2. The Triangular Field Tableau

The index $s$ of $\mathcal{C}_{s}^{k \alpha_{s}}$ or $\overline{\mathcal{C}}_{s \alpha_{s}}^{k}$ labels different horizontal rows, whereas the index $k$ labels different rows slanting to the right and downward.

The new proper solution $S_{\mathrm{nm}}$ of the classical master equation, involving the nonminimal set of fields, is given by

$$
S_{\mathrm{nm}}=S+S_{\mathrm{aux}},
$$


where $S$ is the proper solution for the minimal set of fields presented in Sect. 1 and $S_{\text {aux }}$ is the trivial-pair proper solution for the auxiliary fields given by

$$
\begin{gathered}
S_{\mathrm{aux}}=\bar{\pi}_{0 \alpha_{0}} \overline{\mathcal{C}}_{0}^{* \alpha_{0}}+ \\
\mathcal{C}_{1 \alpha_{1}}^{1 *} \pi_{1}^{1 \alpha_{1}}+\bar{\pi}_{1 \alpha_{1}} \overline{\mathcal{C}}_{1}^{* \alpha_{1}}+ \\
\bar{\pi}_{2 \alpha_{2}}^{2} \overline{\mathcal{C}}_{2}^{2 * \alpha_{2}}+\mathcal{C}_{2 \alpha_{2}}^{1 *} \pi_{2}^{1 \alpha_{2}}+\bar{\pi}_{2 \alpha_{2}} \overline{\mathcal{C}}_{2}^{* \alpha_{2}}+ \\
\mathcal{C}_{3 \alpha_{3}}^{3 *} \pi_{3}^{3 \alpha_{3}}+\bar{\pi}_{3 \alpha_{3}}^{2} \overline{\mathcal{C}}_{3}^{2 * \alpha_{3}}+\mathcal{C}_{3 \alpha_{3}}^{1 *} \pi_{3}^{1 \alpha_{3}}+\bar{\pi}_{3 \alpha_{3}} \overline{\mathcal{C}}_{3}^{* \alpha_{3}}+
\end{gathered}
$$

or

$$
S_{\text {aux }}=\sum_{\substack{k=0 \\ k \text { even }}}^{L} \sum_{s=k}^{L} \bar{\pi}_{s \alpha_{s}}^{k} \overline{\mathcal{C}}_{s}^{k * \alpha_{s}}+\sum_{\substack{k=1 \\ k \text { odd }}}^{L} \sum_{s=k}^{L} \mathcal{C}_{s \alpha_{s}}^{k *} \pi_{s}^{k \alpha_{s}} .
$$

The auxiliary antighost fields at level $s$ are eliminated from the theory using Eq.(6.1) which leads to

$$
\begin{gathered}
\overline{\mathcal{C}}_{s}^{k * \alpha_{s}}=\frac{\partial \Psi}{\partial \overline{\mathcal{C}}_{s \alpha_{s}}^{k}}, \quad k=0,2,4, \ldots, 2\left[\frac{s}{2}\right], \\
\mathcal{C}_{s \alpha_{s}}^{k *}=\frac{\partial \Psi}{\partial \mathcal{C}_{s}^{k \alpha_{s}}}, \quad k=-1,1,3,5, \ldots, 2\left[\frac{s-1}{2}\right]+1,
\end{gathered}
$$

at each level $s$.

\subsection{Delta-Function Gauge-Fixing Procedure}

Let us consider a gauge-fixing scheme for which $\Psi$ is not a function of $\bar{\pi}_{s \alpha_{s}}^{k}$ or $\pi_{s}^{k \alpha_{s}}$ (for $k \geq 0$ ). This is called a $\delta$-function gauge-fixing procedure because when Eq.(6.29) is substituted in $S_{\text {aux }}$ in Eq.(6.28), the conditions

$$
\overline{\mathcal{C}}_{s}^{k * \alpha_{s}}=\frac{\partial \Psi}{\partial \overline{\mathcal{C}}_{s \alpha_{s}}^{k}}=0, \quad \mathcal{C}_{s \alpha_{s}}^{k *}=\frac{\partial \Psi}{\partial \mathcal{C}_{s}^{k \alpha_{s}}}=0
$$

are implemented because the $\pi_{s}^{k \alpha_{s}}$ and $\bar{\pi}_{s \alpha_{s}}^{k}$ in Eq.6.28) act as Lagrange multipliers for these equations. Indeed, in a functional integral approach, integration over $\bar{\pi}_{s \alpha_{s}}^{k}$ and $\pi_{s}^{k \alpha_{s}}$ leads to the insertion of $\delta\left(\frac{\partial \Psi}{\partial \mathcal{C}_{s \alpha_{s}}^{k}}\right)$ and $\delta\left(\frac{\partial \Psi}{\partial \mathcal{C}_{s}^{k \alpha_{s}}}\right)$ in the integrand. Since $\Psi$ does not depend on $\bar{\pi}_{s \alpha_{s}}^{k}$ or $\pi_{s}^{k \alpha_{s}}, \bar{\pi}_{s}^{k * \alpha_{s}}=\frac{\partial \Psi}{\partial \bar{\pi}_{s \alpha_{s}}^{k}}=0$ and $\pi_{s \alpha_{s}}^{k *}=\frac{\partial \Psi}{\partial \pi_{s}^{k \alpha_{s}}}=0$.

Not every $\Psi$ is acceptable. For example, if $\Psi$ is identically zero, all antifields are set to zero and the gauge-fixed proper solution reduces to the original action $S_{0}$. This $S_{0}$ could not be used as the starting point for perturbation theory because propagators 
would not exist. In the rest of this section, we determine the conditions on $\Psi$ so that an admissible gauge-fixing procedure arises. The discussion is somewhat technical so that a reader may wish to skip to Sect. 6.5. For the irreducible case, one should read to Eq. (6.40).

It is useful to define $n_{s}$ as the net number of non-gauge degrees of freedom for the ghost $C_{s}^{\alpha_{s}}$ at level $s$ :

$$
n_{s} \equiv \sum_{t=s}^{L}(-1)^{t-s} m_{t}
$$

The number of gauge degrees of freedom of $\mathcal{C}_{s}^{\alpha_{s}}$ is $m_{s}-n_{s}=n_{s+1}$. If we define $m_{-1}=n$ and use the notation in Eq.(6.23), these statements also apply to $\phi^{i}$ when $s=-1: n_{-1}=m_{-1}-n_{0}=n-n_{0}=n_{\text {dof }}$ is the number of non-gauge degrees of freedom in $\phi^{i}$.

In this paragraph, the general strategy is discussed. The basic idea is that the conditions in Eq.(6.30) fix the gauge degrees of freedom of level $s-1$ fields and nonpropagating independent degrees of freedom of level $s+1$ fields. In the Triangular Field Tableau of Diagram 2, arrows indicate how setting the antifield of one field to zero via Eq.(6.30) eliminates degrees of freedom in another field. An upward-slanting arrow going from $\Phi^{B}$ to $\Phi^{A}$ such as

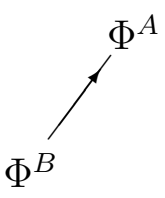

Diagram 3. Elimination of Gauge Degrees of Freedom

indicates that the gauge degrees of freedom in $\Phi^{A}$ are eliminated by the equation $\frac{\partial \Psi}{\partial \Phi_{B}^{*}}=0$. To ensure their elimination, two points on the gauge slice determined by $\Psi$ should not be related by an infinitesimal gauge transformation. This leads to conditions on $\Psi$ that are presented below. A downward-slanting arrow going from $\Phi^{A}$ to $\Phi^{B}$ such as

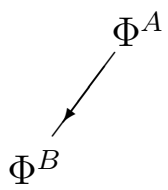

Diagram 4. Elimination of Non-Gauge Degrees of Freedom

indicates that non-propagating independent degrees of freedom in $\Phi^{B}$ are eliminated by the equation $\frac{\partial \Psi}{\partial \Phi_{A}^{*}}=0$. When two arrows appear on a line, there is the fixing of both independent and gauge degrees of freedom. In this delta-function gauge-fixing procedure, only $\phi^{i}, \mathcal{C}_{s}^{\alpha_{s}}$ and $\overline{\mathcal{C}}_{s \alpha_{s}}$ contain propagating fields. In the Triangular Field Tableau of Diagram 2, there are no downward-sloping lines terminating on these fields 
to indicate that non-gauge degrees of freedom are eliminated. In contrast, there are upward-sloping lines meaning that the gauge degrees of freedom in $\phi^{i}, \mathcal{C}_{s}^{\alpha_{s}}$ and $\overline{\mathcal{C}}_{s \alpha_{s}}$ are fixed. The fields $\mathcal{C}_{s}^{k \alpha_{s}}$, for $k \geq 1$ and odd $k$, and $\overline{\mathcal{C}}_{s \alpha_{s}}^{k}$, for $k \geq 2$ and even $k$, are non-propagating, i.e., there is no quadratic form in the action for these fields. When gauge-fixing is fully implemented, they are completely fixed. Like $\mathcal{C}_{s}^{\alpha_{s}}$ and $\overline{\mathcal{C}}_{s \alpha_{s}}$, it will be useful to think of $\mathcal{C}_{s}^{k \alpha_{s}}$ and $\overline{\mathcal{C}}_{s \alpha_{s}}^{k}$ as having $n_{s}$ independent (or non-gauge) degrees of freedom and $n_{s+1}$ gauge degrees of freedom. Since there are both upward-sloping and downward-sloping lines terminating on these fields, both gauge and independent degrees of freedom are fixed. Hence, all $m_{s}=n_{s+1}+n_{s}$ fields are removed from the theory. The difference between $\mathcal{C}_{s}^{\alpha_{s}}$ and $\overline{\mathcal{C}}_{s \alpha_{s}}$ and the other ghosts and antighosts is that the independent degrees of freedom in these fields are propagating. The fields $\overline{\mathcal{C}}_{s \alpha_{s}}$ play a dual role: They fix the gauge degrees of freedom in $\mathcal{C}_{s-1}^{\alpha_{s-1}}$ and serve as the antighosts of $\mathcal{C}_{s}^{\alpha_{s}}$, meaning that they enter quadratically in the action with these fields.

Let $\Phi_{0}$ be a solution to the equations of motion for the action $S_{\Psi}$ fixed by the gauge conditions in Eq.(6.1). Since one is interested in performing a perturbative expansion about this solution, write

$$
\Phi^{A}=\Phi_{0}^{A}+\delta \Phi^{A},
$$

where $\delta \Phi^{A}$ is the quantum fluctuation of $\Phi^{A}$. According to the Triangular Field Tableau of Diagram 2, the gauge degrees of freedom of $\phi^{i}$ should be fixed by the equation $0=\overline{\mathcal{C}}_{0}^{* \alpha_{0}}$. Expanding about the perturbative solution, one obtains

$$
0=\overline{\mathcal{C}}_{0}^{* \alpha_{0}}=\frac{\partial \Psi}{\partial \overline{\mathcal{C}}_{0 \alpha_{0}}}=\left(\frac{\partial \Psi}{\partial \overline{\mathcal{C}}_{0 \alpha_{0}}}\right)_{0}+\left(\frac{\partial_{l} \partial_{r} \Psi}{\partial \overline{\mathcal{C}}_{0 \alpha_{0}} \partial \phi^{i}}\right)_{0} \delta \phi^{i}+\ldots,
$$

where we use the abbreviation

$$
\left.()_{0} \equiv()\right|_{\left\{\Phi^{A}=\Phi_{0}^{A}\right\}}
$$

The matrix $\left(\frac{\partial_{l} \partial_{r} \Psi}{\partial \mathcal{C}_{0} \alpha_{0} \partial \phi^{i}}\right)$ that multiplies $\delta \phi^{i}$ must eliminate $n_{0}$ gauge degrees of freedom. This requires that $\Psi$ be such that

$$
\operatorname{rank}\left(\frac{\partial_{l} \partial_{r} \Psi}{\partial \overline{\mathcal{C}}_{0 \alpha_{0}} \partial \phi^{i}}\right)_{0}=n_{0}
$$

The gauge modes of $\phi^{i}$ are proportional to $R_{\alpha_{0}}^{i}$. Hence one also needs

$$
\operatorname{rank}\left(\frac{\partial_{l} \partial_{r} \Psi}{\partial \overline{\mathcal{C}}_{0 \alpha_{0}} \partial \phi^{i}} R_{\beta_{0}}^{i}\right)_{0}=n_{0}
$$


to ensure that the correct degrees of freedom in $\phi^{i}$ are fixed. Using Eq.(4.28) and $\phi_{i}^{*}=\frac{\partial \Psi}{\partial \phi^{i}}$, one sees that the operator in Eq.(6.36) is the quadratic form for $\overline{\mathcal{C}}_{0 \alpha_{0}}$ and $\mathcal{C}_{0}^{\beta_{0}}$ in the action $S_{\Psi}$ :

$$
S_{\Psi}=\ldots+\overline{\mathcal{C}}_{0 \alpha_{0}}\left(\frac{\partial_{l} \partial_{r} \Psi}{\partial \overline{\mathcal{C}}_{0 \alpha_{0}} \partial \phi^{i}} R_{\beta_{0}}^{i}\right)_{0} \mathcal{C}_{0}^{\beta_{0}}+\ldots .
$$

Hence Eq. (6.36) is consistent with the fact that $\overline{\mathcal{C}}_{0 \alpha_{0}}$ and $\mathcal{C}_{0}^{\beta_{0}}$ should have $n_{0}$ propagating degrees of freedom.

If the system is irreducible, $n_{0}=m_{0}$ and no further constraints on the gaugefixing fermion $\Psi$ are necessary. Eqs.6.35) and (6.36) are the only requirements on $\Psi$. There are $n-m_{0}$ propagating modes for $\phi^{i}$ and $m_{0}$ propagating modes for $\overline{\mathcal{C}}_{0 \alpha_{0}}$ and $\mathcal{C}_{0}^{\alpha_{0}}$. Of the original $n$ degrees of freedom of $\phi^{i}, m_{0}$ have been gauge fixed. The simplest gauge-fixing fermion $\Psi_{\delta}$ is 26]

$$
\Psi_{\delta}=\overline{\mathcal{C}}_{0 \alpha_{0}} \chi^{\alpha_{0}}(\phi)
$$

where $\chi^{\alpha_{0}}$ is an arbitrary functional of $\phi$. The subscript $\delta$ on $\Psi$ indicates that this is a delta-function gauge-fixing scheme. The condition $0=\overline{\mathcal{C}}_{0}^{* \alpha_{0}}$ in Eq.(6.33) leads to

$$
\chi^{\alpha_{0}}(\phi)=0,
$$

so that $\chi^{\alpha_{0}}$ is the gauge-fixing condition on $\phi$. Eqs.(6.35) and (6.36) respectively become

$$
\begin{gathered}
\operatorname{rank}\left(\chi_{, i}^{\alpha_{0}}\left(\phi_{0}\right)\right)=n_{0}, \\
\operatorname{rank}\left(\chi_{, i}^{\alpha_{0}} R_{\beta_{0}}^{i}\left(\phi_{0}\right)\right)=n_{0},
\end{gathered}
$$

where $n_{0}=m_{0}$ for the irreducible case.

Suppose the theory is at least first-stage reducible. Then $\overline{\mathcal{C}}_{0 \alpha_{0}}$ and $\mathcal{C}_{0}^{\alpha_{0}}$ have $m_{0}$ degrees of freedom but $n_{1}$ of these are gauge modes and thus $m_{0}-n_{1}=n_{0}$ are independent. According to the Triangular Field Tableau of Diagram 2, the equation $\overline{\mathcal{C}}_{1}^{* \alpha_{1}}=0$ is used to fix the $n_{1}$ gauge degrees of freedom in $\mathcal{C}_{0}^{\alpha_{0}}$, and $\mathcal{C}_{1 \alpha_{1}}^{1 *}=0$ is used to fix the $n_{1}$ gauge degrees of freedom in $\overline{\mathcal{C}}_{0 \alpha_{0}}$. Expanding the former condition about the perturbative solution, one obtains

$$
0=\overline{\mathcal{C}}_{1}^{* \alpha_{1}}=\frac{\partial \Psi}{\partial \overline{\mathcal{C}}_{1 \alpha_{1}}}=\left(\frac{\partial \Psi}{\partial \overline{\mathcal{C}}_{1 \alpha_{1}}}\right)_{0}+\left(\frac{\partial_{l} \partial_{r} \Psi}{\partial \overline{\mathcal{C}}_{1 \alpha_{1}} \partial \mathcal{C}_{0}^{\alpha_{0}}}\right)_{0} \delta \mathcal{C}_{0}^{\alpha_{0}}+\ldots
$$

Actually, the first term on the right-hand side of Eq.(6.41) vanishes, but we display it to emphasize the idea that we are expanding about the perturbative solution. Since one wants to fix $n_{1}$ gauge modes of $\mathcal{C}_{0}^{\alpha_{0}}$, a necessary condition on $\Psi$ is that

$$
\operatorname{rank}\left(\frac{\partial_{l} \partial_{r} \Psi}{\partial \overline{\mathcal{C}}_{1 \alpha_{1}} \partial \mathcal{C}_{0}^{\alpha_{0}}}\right)_{0}=n_{1}
$$


The modes to gauge-fix are those that are non-propagating in Eq.(6.37). These modes are proportional to

$$
\left.Z_{1 \beta_{1}}^{-1 \alpha_{0}} \equiv R_{1 \beta_{1}}^{\alpha_{0}}\right|_{0} \quad
$$

of Eq.(2.16). Since there are $n_{1}$ such modes,

$$
\operatorname{rank}\left(Z_{1 \beta_{1}}^{-1 \alpha_{0}}\right)=n_{1}
$$

Because these modes need to be eliminated by the $0=\overline{\mathcal{C}}_{1}^{* \alpha_{1}}$ condition in Eq. (6.41), they should not be annihilated by the quadratic form $\left(\frac{\partial_{l} \partial_{r} \Psi}{\partial \mathcal{C}_{1 \alpha_{1}} \partial \mathcal{C}_{0}^{\alpha}}\right)_{0}$ in Eq. (6.42). This leads to the condition on $\Psi$ that

$$
\operatorname{rank}\left(\frac{\partial_{l} \partial_{r} \Psi}{\partial \overline{\mathcal{C}}_{1 \alpha_{1}} \partial \mathcal{C}_{0}^{\alpha_{0}}} Z_{1 \beta_{1}}^{-1 \alpha_{0}}\right)_{0}=n_{1}
$$

Using $\mathcal{C}_{0 \alpha_{0}}^{*}=\frac{\partial_{r} \Psi}{\partial \mathcal{C}_{0}^{\alpha_{0}}}$ in Eq. (4.28) and Eq.(6.43), one notices that the operator in Eq.(6.45) is the quadratic form for $\overline{\mathcal{C}}_{1 \alpha_{1}}$ and $\mathcal{C}_{1}^{\beta_{1}}$. Since these fields have $n_{1}$ propagating degrees of freedom, the rank of the quadratic form should be $n_{1}$, which is consistent with Eq.(6.45).

To fix the $n_{1}$ gauge modes of $\overline{\mathcal{C}}_{0 \alpha_{0}}$, use

$$
0=\mathcal{C}_{1 \alpha_{1}}^{1 *}=\frac{\partial \Psi}{\partial \mathcal{C}_{1}^{1 \alpha_{1}}}=\left(\frac{\partial \Psi}{\partial \mathcal{C}_{1}^{1 \alpha_{1}}}\right)_{0}+\delta \overline{\mathcal{C}}_{0 \alpha_{0}}\left(\frac{\partial_{l} \partial_{r} \Psi}{\partial \overline{\mathcal{C}}_{0 \alpha_{0}} \partial \mathcal{C}_{1}^{1 \alpha_{1}}}\right)_{0}+\ldots
$$

A necessary condition that these gauge degrees of freedom can be fixed is

$$
\operatorname{rank}\left(\frac{\partial_{l} \partial_{r} \Psi}{\partial \overline{\mathcal{C}}_{0 \alpha_{0}} \partial \mathcal{C}_{1}^{1 \alpha_{1}}}\right)_{0}=n_{1}
$$

Next, note that, since rank $\left(\frac{\partial_{1} \partial_{r} \Psi}{\partial \mathcal{C}_{0} \alpha_{0} \partial \phi^{2}}\right)_{0}=n_{0}$ (see Eq. 6.35)) and the index $\alpha_{0}$ takes on $m_{0}$ values, there must be $m_{0}-n_{0}=n_{1}$ left zero modes $\bar{Z}_{1 \alpha_{0}}^{0 \alpha_{1}}$ for the operator $\left(\frac{\partial_{l} \partial_{r} \Psi}{\partial \mathcal{C}_{0} \alpha_{0} \partial \phi^{2}}\right)_{0}:$

$$
\bar{Z}_{1 \alpha_{0}}^{0 \alpha_{1}}\left(\frac{\partial_{l} \partial_{r} \Psi}{\partial \overline{\mathcal{C}}_{0 \alpha_{0}} \partial \phi^{i}}\right)_{0}=0
$$

where we use a redundant superscript $\alpha_{1}$ on $\bar{Z}_{1 \alpha_{0}}^{0 \alpha_{1}}$ but compensate for this by requiring

$$
\operatorname{rank}\left(\bar{Z}_{1 \alpha_{0}}^{0 \alpha_{1}}\right)=n_{1}
$$

The $\bar{Z}_{1 \alpha_{0}}^{0 \alpha_{1}}$ are the non-propagating modes of $\overline{\mathcal{C}}_{0 \alpha_{0}}$ in the quadratic form in Eq. (6.37). They must be fixed from the $0=\mathcal{C}_{1 \alpha_{1}}^{1 *}$ condition in Eq.(6.46), so that

$$
\operatorname{rank}\left(\bar{Z}_{1 \beta_{0}}^{0 \alpha_{1}} \frac{\partial_{l} \partial_{r} \Psi}{\partial \overline{\mathcal{C}}_{0 \alpha_{0}} \partial \mathcal{C}_{1}^{1 \alpha_{1}}}\right)_{0}=n_{1}
$$


Finally one needs to fix the $n_{1}$ independent or "non-gauge" modes of $\mathcal{C}_{1}^{1 \alpha_{1}}$. From Diagram 2, one sees that this is done using

$$
0=\overline{\mathcal{C}}_{0}^{* \alpha_{0}}=\frac{\partial \Psi}{\partial \overline{\mathcal{C}}_{0 \alpha_{0}}}=\left(\frac{\partial \Psi}{\partial \overline{\mathcal{C}}_{0 \alpha_{0}}}\right)_{0}+\left(\frac{\partial_{l} \partial_{r} \Psi}{\partial \overline{\mathcal{C}}_{0 \alpha_{0}} \partial \mathcal{C}_{1}^{1 \alpha_{1}}}\right)_{0} \delta \mathcal{C}_{1}^{1 \alpha_{1}}+\ldots
$$

for which one needs

$$
\operatorname{rank}\left(\frac{\partial_{l} \partial_{r} \Psi}{\partial \overline{\mathcal{C}}_{0 \alpha_{0}} \partial \mathcal{C}_{1}^{1 \alpha_{1}}}\right)_{0}=n_{1}
$$

which is the same condition as Eq. (6.47).

If the system is first-stage reducible then $n_{1}=m_{1}$ and there are no gauge degrees of freedom in $\mathcal{C}_{1}^{1 \alpha_{1}}$ so that the above condition eliminates all the modes of $\mathcal{C}_{1}^{1 \alpha_{1}}$. For $\phi^{i}$, one has $n-m_{0}+m_{1}$ propagating modes and $m_{0}-m_{1}$ gauge modes fixed by gauge conditions. For $\overline{\mathcal{C}}_{0 \alpha_{0}}$ and $\mathcal{C}_{0}^{\alpha_{0}}$, there are $m_{0}-m_{1}$ propagating modes and the $m_{1}$ gauge degrees of freedom are fixed. Lastly, all $m_{1}$ degrees of freedom of $\overline{\mathcal{C}}_{1 \alpha_{1}}$ and of $\mathcal{C}_{1}^{\alpha_{1}}$ are propagating. Summarizing, for a first-stage reducible theory the conditions on $\Psi$ are given in Eqs.(6.35), (6.36), 6.42), (6.45), 6.47), and (6.50).

An example of an acceptable $\Psi_{\delta}$ is 26

$$
\Psi_{\delta}=\overline{\mathcal{C}}_{0 \alpha_{0}} \chi^{\alpha_{0}}(\phi)+\overline{\mathcal{C}}_{1 \alpha_{1}} \omega_{1 \alpha_{0}}^{0 \alpha_{1}}(\phi) \mathcal{C}_{0}^{\alpha_{0}}+\overline{\mathcal{C}}_{0 \alpha_{0}} \bar{\omega}_{0 \alpha_{1}}^{1 \alpha_{0}}(\phi) \mathcal{C}_{1}^{1 \alpha_{1}}
$$

where $\chi^{\alpha_{0}}, \omega_{1 \alpha_{0}}^{0 \alpha_{1}}$ and $\bar{\omega}_{0 \alpha_{1}}^{1 \alpha_{0}}$ are arbitrary functionals of $\phi$ subject to Eq.(6.40) and

$$
\begin{aligned}
& \operatorname{rank}\left(\omega_{1 \alpha_{0}}^{0 \alpha_{1}}\left(\phi_{0}\right)\right)=n_{1}, \\
& \operatorname{rank}\left(\omega_{1 \alpha_{0}}^{0 \alpha_{1}}\left(\phi_{0}\right) Z_{1 \beta_{1}}^{-1 \alpha_{0}}\right)=n_{1}, \\
& \operatorname{rank}\left(\bar{\omega}_{0 \alpha_{1}}^{1 \alpha_{0}}\left(\phi_{0}\right)\right)=n_{1}, \\
& \operatorname{rank}\left(\bar{Z}_{1 \alpha_{0}}^{0 \alpha_{1}} \bar{\omega}_{0 \alpha_{1}}^{1 \alpha_{0}}\left(\phi_{0}\right)\right)=n_{1},
\end{aligned}
$$

where $n_{1}=m_{1}$ for first-stage reducible theories. The $Z_{1 \beta_{1}}^{-1 \alpha_{0}}$ are given in Eq. (6.43) and $\bar{Z}_{1 \alpha_{0}}^{0 \alpha_{1}}$ is determined by $\bar{Z}_{1 \alpha_{0}}^{0 \alpha_{1}} \chi_{, i}^{\alpha_{0}}\left(\phi_{0}\right)=0$.

For completeness, we present the conditions on $\Psi$ for the general Lth-stage reducible theory. There are fields from levels $s=-1$ to $s=L$. See Triangular Field Tableau of Diagram 2.

For odd $k$ and $-1 \leq k \leq s$, there are conditions fixing the $n_{s+1}$ gauge degrees of $\mathcal{C}_{s}^{k \alpha_{s}}$ coming from the diagram

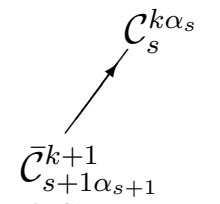

Diagram 5. Elimination of Gauge Degrees of Freedom in $\mathcal{C}_{s}^{k \alpha_{s}}$ 
and arising from

$$
0=\overline{\mathcal{C}}_{s+1}^{k+1 * \alpha_{s+1}}=\frac{\partial \Psi}{\partial \overline{\mathcal{C}}_{s+1 \alpha_{s+1}}^{k+1}}=\ldots+\left(\frac{\partial_{l} \partial_{r} \Psi}{\partial \overline{\mathcal{C}}_{s+1 \alpha_{s+1}}^{k+1} \partial \mathcal{C}_{s}^{k \alpha_{s}}}\right)_{0} \delta \mathcal{C}_{s}^{k \alpha_{s}}+\ldots
$$

A necessary condition that the $n_{s+1}$ gauge modes of $\mathcal{C}_{s}^{k \alpha_{s}}$ can be fixed is

$$
\operatorname{rank}\left(\frac{\partial_{l} \partial_{r} \Psi}{\partial \overline{\mathcal{C}}_{s+1 \alpha_{s+1}}^{k+1} \partial \mathcal{C}_{s}^{k \alpha_{s}}}\right)_{0}=n_{s+1}
$$

where $k$ is odd and ranges between $s \geq k \geq-1$, and $s$ is restricted to $L-1 \geq s \geq-1$.

When there is a diagram such as

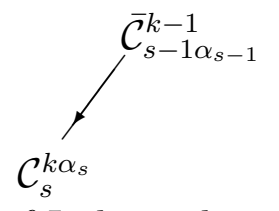

Diagram 6. Elimination of Independent Degrees of Freedom in $\mathcal{C}_{s}^{k \alpha_{s}}$ the remaining $n_{s}$ independent degrees of freedom in $\mathcal{C}_{s}^{k \alpha_{s}}$ are fixed using

$$
0=\overline{\mathcal{C}}_{s-1}^{k-1 * \alpha_{s-1}}=\frac{\partial \Psi}{\partial \overline{\mathcal{C}}_{s-1 \alpha_{s-1}}^{k-1}}=\ldots+\left(\frac{\partial_{l} \partial_{r} \Psi}{\partial \overline{\mathcal{C}}_{s-1 \alpha_{s-1}}^{k-1} \partial \mathcal{C}_{s}^{k \alpha_{s}}}\right)_{0} \delta \mathcal{C}_{s}^{k \alpha_{s}}+\ldots
$$

A necessary condition that these $n_{s}$ non-gauge modes can be fixed is

$$
\operatorname{rank}\left(\frac{\partial_{l} \partial_{r} \Psi}{\partial \overline{\mathcal{C}}_{s-1 \alpha_{s-1}}^{k-1} \partial \mathcal{C}_{s}^{k \alpha_{s}}}\right)_{0}=n_{s}
$$

where $k$ is odd, $s \geq k \geq 1$, and $L \geq s \geq 1$.

The gauge modes of $\mathcal{C}_{s}^{k \alpha_{s}}$ are those not fixed by the condition in Eq.(6.56). They are annihilated by the quadratic form $\left(\frac{\partial_{l} \partial_{r} \Psi}{\partial \overline{\mathcal{C}}_{s-1 \alpha_{s-1}}^{k-1} \partial \mathcal{C}_{s}^{k \alpha_{s}}}\right)_{0}$ in Eq.(6.56). Since this operator acts on $m_{s}$-component vectors and has rank $n_{s}$, it must have exactly $m_{s}-n_{s}=n_{s+1}$ right null vectors. We denote these by $Z_{s+1 \beta_{s+1}}^{k \alpha_{s}}$ using the redundant subscript index $\beta_{s+1}$ but requiring

$$
\operatorname{rank}\left(Z_{s+1 \beta_{s+1}}^{k \alpha_{s}}\right)=n_{s+1}
$$

More precisely,

$$
\left(\frac{\partial_{l} \partial_{r} \Psi}{\partial \overline{\mathcal{C}}_{s-1 \alpha_{s-1}}^{k-1} \partial \mathcal{C}_{s}^{k \alpha_{s}}}\right)_{0} Z_{s+1 \beta_{s+1}}^{k \alpha_{s}}=0
$$

A sufficient condition that these gauge modes $Z_{s+1 \beta_{s+1}}^{k \alpha_{s}}$ can be removed from $\mathcal{C}_{s}^{k \alpha_{s}}$ via Eq. (6.54) is that

$$
\operatorname{rank}\left(\frac{\partial_{l} \partial_{r} \Psi}{\partial \overline{\mathcal{C}}_{s+1 \alpha_{s+1}}^{k+1} \partial \mathcal{C}_{s}^{k \alpha_{s}}} Z_{s+1 \beta_{s+1}}^{k \alpha_{s}}\right)_{0}=n_{s+1}
$$


The conditions on $\Psi$ in Eq.(6.60) hold for odd $k, s \geq k \geq-1$ and $L-1 \geq s \geq-1$ if one defines

$$
\left.Z_{s+1 \beta_{s+1}}^{-1 \alpha_{s}} \equiv R_{s+1 \beta_{s+1}}^{\alpha_{s}}\right|_{0}
$$

When $k=-1$ the operator in Eq. (6.60) is the quadratic form of $S_{\Psi}$ for $\overline{\mathcal{C}}_{s+1 \alpha_{s+1}}$ and $\mathcal{C}_{s+1}^{\beta_{s+1}}$, as can be seen from Eqs. (4.28) and (6.29). The condition that these fields have $n_{s+1}$ propagating modes is consistent with Eq.(6.60) for the case $k=-1$.

The discussion for $\overline{\mathcal{C}}_{s \alpha_{s}}^{k}$ where $k$ is even works similarly. The $n_{s}$ independent degrees of freedom of $\overline{\mathcal{C}}_{s \alpha_{s}}$, via the diagram

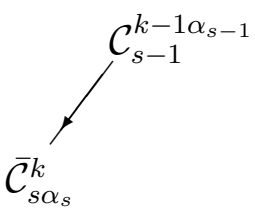

Diagram 7. Elimination of Independent Degrees of Freedom in $\overline{\mathcal{C}}_{s \alpha_{s}}^{k}$

are fixed by

$$
0=\mathcal{C}_{s-1 \alpha_{s-1}}^{k-1 *}=\frac{\partial \Psi}{\partial \mathcal{C}_{s-1}^{k-1 \alpha_{s-1}}}=\ldots+\delta \overline{\mathcal{C}}_{s \alpha_{s}}^{k}\left(\frac{\partial_{l} \partial_{r} \Psi}{\partial \overline{\mathcal{C}}_{s \alpha_{s}}^{k} \partial \mathcal{C}_{s-1}^{k-1 \alpha_{s-1}}}\right)_{0}+\ldots
$$

which requires

$$
\operatorname{rank}\left(\frac{\partial_{l} \partial_{r} \Psi}{\partial \overline{\mathcal{C}}_{s \alpha_{s}}^{k} \partial \mathcal{C}_{s-1}^{k-1 \alpha_{s-1}}}\right)_{0}=n_{s}
$$

This is the same condition as in Eq.(6.55) when $s \rightarrow s+1$ and $k \rightarrow k+1$.

The gauge modes of $\overline{\mathcal{C}}_{s \alpha_{s}}^{k}$ are the left zero modes of the operator $\left(\frac{\partial_{l} \partial_{r} \Psi}{\partial \overline{\mathcal{C}}_{s \alpha_{s}}^{k} \partial \mathcal{C}_{s-1}^{k-1 \alpha_{s-1}}}\right)_{0}$ in Eq.(6.62). Since this operator acts to the left on $m_{s}$-component vectors and has rank $n_{s}$, there are exactly $m_{s}-n_{s}=n_{s+1}$ such zero modes. We denote these by $\bar{Z}_{s+1 \alpha_{s}}^{k \alpha_{s+1}}$, using the redundant superscript index $\alpha_{s+1}$ but requiring

$$
\operatorname{rank}\left(\bar{Z}_{s+1 \alpha_{s}}^{k \alpha_{s+1}}\right)=n_{s+1}
$$

More precisely, the $\bar{Z}_{s+1 \alpha_{s}}^{k \alpha_{s+1}}$ are defined by

$$
\bar{Z}_{s+1 \alpha_{s}}^{k \alpha_{s+1}}\left(\frac{\partial_{l} \partial_{r} \Psi}{\partial \overline{\mathcal{C}}_{s \alpha_{s}}^{k} \partial \mathcal{C}_{s-1}^{k-1 \alpha_{s-1}}}\right)_{0}=0
$$

where $k$ is even, $s-1 \geq k \geq 0$, and $L-1 \geq s \geq 0$. These $n_{s+1}$ gauge degrees of freedom of $\overline{\mathcal{C}}_{s \alpha_{s}}^{k}$, via the diagram 


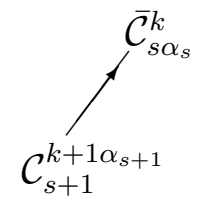

Diagram 8. Elimination of Gauge Degrees of Freedom in $\overline{\mathcal{C}}_{s \alpha_{s}}^{k}$

are fixed by

$$
0=\mathcal{C}_{s+1 \alpha_{s+1}}^{k+1 *}=\frac{\partial \Psi}{\partial \mathcal{C}_{s+1}^{k+1 \alpha_{s+1}}}=\ldots+\delta \overline{\mathcal{C}}_{s \alpha_{s}}^{k}\left(\frac{\partial_{l} \partial_{r} \Psi}{\partial \overline{\mathcal{C}}_{s \alpha_{s}}^{k} \partial \mathcal{C}_{s+1}^{k+1 \alpha_{s+1}}}\right)_{0}+\ldots
$$

which requires

$$
\operatorname{rank}\left(\frac{\partial_{l} \partial_{r} \Psi}{\partial \overline{\mathcal{C}}_{s \alpha_{s}}^{k} \partial \mathcal{C}_{s+1}^{k+1 \alpha_{s+1}}}\right)_{0}=n_{s+1}
$$

This is the same condition as in Eq.(6.57) when $s \rightarrow s-1$ and $k \rightarrow k-1$. Since these gauge modes are proportional to $\bar{Z}_{s+1 \alpha_{s}}^{k \beta_{s+1}}$, a sufficient condition for the elimination of these modes is

$$
\operatorname{rank}\left(\bar{Z}_{s+1 \alpha_{s}}^{k \beta_{s+1}} \frac{\partial_{l} \partial_{r} \Psi}{\partial \overline{\mathcal{C}}_{s \alpha_{s}}^{k} \partial \mathcal{C}_{s+1}^{k+1 \alpha_{s+1}}}\right)_{0}=n_{s+1}
$$

where $k$ is even, $s-1 \geq k \geq 0$, and $L-1 \geq s \geq 0$.

As a final remark, note that all the gauge-fixing conditions have been used: The equations $0=\mathcal{C}_{s \alpha_{s}}^{k *}$ (and $0=\overline{\mathcal{C}}_{s}^{k * \alpha_{s}}$ ) impose $n_{s}$ constraints on level $s-1$ gauge fields and $n_{s+1}$ constraints on level $s+1$ independent fields. The total number of constraints imposed is the number of values of the index $\alpha_{s}$. This number is $m_{s}$ and equals $n_{s}+n_{s+1}$.

For an $L$ th-stage reducible theory, it is necessary for $\Psi$ to satisfy Eqs. 6.55), (6.57), (6.60) and (6.66). All degrees of freedom in $\mathcal{C}_{s}^{k \alpha_{s}}$ for $k$ odd and $k \geq 1$ and in $\overline{\mathcal{C}}_{s \alpha_{s}}^{k}$ for $k$ even and $k \geq 2$ are fixed. Of the original $\phi^{i}, n_{0}$ of the degrees of freedom are fixed and the remaining $n-n_{0}$ modes are propagating. For $\overline{\mathcal{C}}_{s \alpha_{s}}$ and $\mathcal{C}_{s}^{\alpha_{s}}$ with $0 \leq s \leq L-1, n_{s}$ fields are propagating and $n_{s+1}$ fields have been gauge-fixed. For $s=L$, all $n_{L}=m_{L}$ modes of $\overline{\mathcal{C}}_{L \alpha_{L}}$ and $\mathcal{C}_{L}^{\alpha_{L}}$ are propagating.

One useful example of a gauge-fixing fermion is [26]

$$
\begin{gathered}
\Psi_{\delta}=\overline{\mathcal{C}}_{0 \alpha_{0}} \chi^{\alpha_{0}}(\phi)+ \\
\sum_{s=1}^{L} \sum_{\substack{k=0 \\
k \text { even }}}^{s} \overline{\mathcal{C}}_{s \alpha_{s}}^{k} \omega_{s \alpha_{s-1}}^{k \alpha_{s}}(\phi) \mathcal{C}_{s-1}^{k-1 \alpha_{s-1}}+\sum_{s=1}^{L} \sum_{\substack{k=1 \\
k \text { odd }}}^{s} \overline{\mathcal{C}}_{s-1 \alpha_{s-1}}^{k-1} \bar{\omega}_{s \alpha_{s}}^{k \alpha_{s-1}}(\phi) \mathcal{C}_{s}^{k \alpha_{s}}
\end{gathered}
$$

where, for each upward-sloping line (like in Diagram 5 with $k \rightarrow k-1$ and $s \rightarrow s-1$ ), one associates the matrix $\omega_{s \alpha_{s-1}}^{k \alpha_{s}}$ and where, for each downward-sloping line (like in 
Diagram 6), one associates the matrix $\bar{\omega}_{s \alpha_{s}}^{k \alpha_{s-1}}$. In addition to Eq.(6.40), one requires Eqs. 6.55), (6.60), Eq.(6.57), and (6.66), that is,

$$
\begin{gathered}
\operatorname{rank}\left(\omega_{s \alpha_{s-1}}^{k \alpha_{s}}\left(\phi_{0}\right)\right)=n_{s}, \\
\operatorname{rank}\left(\omega_{s \alpha_{s-1}}^{k \alpha_{s}}\left(\phi_{0}\right) Z_{s \beta_{s}}^{k-1 \alpha_{s-1}}\right)=n_{s},
\end{gathered}
$$

for $k$ even, $s \geq k \geq 0$ and $L \geq s \geq 1$, as well as

$$
\begin{gathered}
\operatorname{rank}\left(\bar{\omega}_{s \alpha_{s}}^{k \alpha_{s-1}}\left(\phi_{0}\right)\right)=n_{s}, \\
\operatorname{rank}\left(\bar{Z}_{s \alpha_{s-1}}^{k-1 \beta_{s}} \bar{\omega}_{s \alpha_{s}}^{k \alpha_{s-1}}\left(\phi_{0}\right)\right)=n_{s},
\end{gathered}
$$

for $k$ odd, $s \geq k \geq 1$ and $L \geq s \geq 1$.

\subsection{Other Gauge-Fixing Procedures}

The previous subsection considered the most general gauge-fixing fermion $\Psi$ which is independent of $\bar{\pi}_{s \alpha_{s}}^{k}$ and $\pi_{s}^{k \alpha_{s}}$. By allowing $\Psi$ to be linear in $\bar{\pi}_{s \alpha_{s}}^{k}$ and $\pi_{s}^{k \alpha_{s}}$, one is able to have gaussian averaging over a gauge condition [26]. Such possibilities arise when

$$
\Psi=\Psi_{\delta}+\Psi_{\pi}
$$

where $\Psi_{\delta}$ is a $\delta$-function-type gauge-fixing fermion which is independent of $\bar{\pi}_{s \alpha_{s}}^{k}$ and $\pi_{s}^{k \alpha_{s}}$, and where $\Psi_{\pi}$ is linear in $\bar{\pi}_{s \alpha_{s}}^{k}$ and $\pi_{s}^{k \alpha_{s}}$.

For example, in an irreducible theory, one may take

$$
\Psi_{\pi}=\frac{1}{2} \overline{\mathcal{C}}_{0 \alpha_{0}} \sigma_{0}^{0 \alpha_{0} \beta_{0}}(\phi) \bar{\pi}_{0 \beta_{0}}
$$

where $\sigma_{0}^{0 \alpha_{0} \beta_{0}}$ is an arbitrary matrix. The total gauge-fixing fermion $\Psi$ is

$$
\Psi=\overline{\mathcal{C}}_{0 \alpha_{0}} \chi^{\alpha_{0}}(\phi)+\frac{1}{2} \overline{\mathcal{C}}_{0 \alpha_{0}} \sigma_{0}^{0 \alpha_{0} \beta_{0}}(\phi) \bar{\pi}_{0 \beta_{0}}
$$

The antifields $\overline{\mathcal{C}}_{0}^{* \alpha_{0}}$ are eliminated via Eq. (6.1) to give

$$
\overline{\mathcal{C}}_{0}^{* \alpha_{0}}=\chi^{\alpha_{0}}+\frac{1}{2} \sigma_{0}^{0 \alpha_{0} \beta_{0}} \bar{\pi}_{0 \beta_{0}}
$$

which, when substituted into Eq.(6.28), results in

$$
\left.S_{\mathrm{aux}}\right|_{\Sigma_{\Psi}}=\bar{\pi}_{0 \alpha_{0}} \chi^{\alpha_{0}}(\phi)+\frac{1}{2} \bar{\pi}_{0 \alpha_{0}} \sigma_{0}^{0 \alpha_{0} \beta_{0}} \bar{\pi}_{0 \beta_{0}}
$$

Without the quadratic functional of $\bar{\pi}_{0 \alpha_{0}}$, Eq. 6.74) would give the gauge-fixing conditions $\chi^{\alpha_{0}}(\phi)=0$ on $\phi$. With the quadratic functional of $\bar{\pi}_{0 \alpha_{0}}$, one is able to perform 
a gaussian averaging over these gauging-fixing conditions. If the matrix $\sigma_{0}^{0 \alpha_{0} \beta_{0}}$ is completely invertible then the averaging is done over all gauge invariances. By choosing $\sigma_{0}^{0 \alpha_{0} \beta_{0}}$ not to have an inverse, one may obtain delta-function conditions for some gauge invariances and a gaussian-averaging of others. In some quantization schemes, $\bar{\pi}_{0 \alpha_{0}}$ may become propagating, in which case $\bar{\pi}_{0 \alpha_{0}}$ is called a Nielsen-Kallosh ghost 172, 199, 200.

For a first-stage reducible theory, one can add

$$
\Psi_{\pi}=\frac{1}{2} \overline{\mathcal{C}}_{0 \alpha_{0}} \sigma_{0}^{0 \alpha_{0} \beta_{0}} \bar{\pi}_{0 \beta_{0}}+\frac{1}{2} \overline{\mathcal{C}}_{1 \alpha_{1}} \rho_{1 \beta_{1}}^{0 \alpha_{1}} \pi_{1}^{1 \beta_{1}}+\frac{1}{2} \bar{\pi}_{1 \alpha_{1}} \rho_{1 \beta_{1}}^{0 \alpha_{1}} \mathcal{C}_{1}^{1 \beta_{1}}
$$

to the $\delta$-function gauge-fixing fermion $\Psi_{\delta}$ in Eq.(6.52), where $\sigma_{0}^{0 \alpha_{0} \beta_{0}}$ and $\rho_{1 \beta_{1}}^{0 \alpha_{1}}$ are arbitrary matrix functionals of $\phi$. Eliminating antifields by Eq.(6.1) and inserting into Eq. (6.28) leads to

$$
\left.S_{\mathrm{aux}}\right|_{\Sigma_{\Psi}}=\ldots+\frac{1}{2} \bar{\pi}_{0 \alpha_{0}} \sigma_{0}^{0 \alpha_{0} \beta_{0}} \bar{\pi}_{0 \beta_{0}}+\bar{\pi}_{1 \alpha_{0}} \rho_{1 \beta_{1}}^{0 \alpha_{1}} \pi_{1}^{1 \beta_{1}}+\ldots
$$

For appropriate $\sigma_{0}^{0 \alpha_{0} \beta_{0}}$ and $\rho_{1 \beta_{1}}^{0 \alpha_{1}}$, integration over $\bar{\pi}_{0 \alpha_{0}}, \pi_{1}^{1 \beta_{1}}$ and $\bar{\pi}_{1 \alpha_{0}}$ leads to a gaussian averaging gauge-fixing procedure.

For an $L$ th-stage system, the most general $\Psi_{\pi}$, satisfying Eq.(6.4) and being linear in $\pi_{s}^{k \alpha_{s}}$ and $\bar{\pi}_{s \alpha_{s}}^{k}$ and linear in the auxiliary ghosts and antighosts, is

$$
\begin{aligned}
\Psi_{\pi}=\frac{1}{2} \sum_{\substack{k=0 \\
k \text { even }}}^{L-1} \sum_{s=k+1}^{L}\left(\overline{\mathcal{C}}_{s \alpha_{s}}^{k} \rho_{s \beta_{s}}^{k \alpha_{s}}(\phi) \pi_{s}^{k+1 \beta_{s}}+\bar{\pi}_{s \alpha_{s}}^{k} \rho_{s \beta_{s}}^{k \alpha_{s}}(\phi) \mathcal{C}_{s}^{k+1 \beta_{s}}\right) \\
+\frac{1}{2} \sum_{\substack{k=0 \\
k \text { even }}}^{L} \overline{\mathcal{C}}_{k \alpha_{k}}^{k} \sigma_{k}^{k \alpha_{k} \beta_{k}}(\phi) \bar{\pi}_{k \beta_{k}}^{k},
\end{aligned}
$$

where $\rho_{s \beta_{s}}^{k \alpha_{s}}$ and $\sigma_{k}^{k \alpha_{k} \beta_{k}}$ are arbitrary matrices. If one eliminates antifields by Eq.(6.1) and inserts the result into Eq.(6.28), one obtains the following quadratic terms for $\pi$ fields

$$
\begin{gathered}
\left.S_{\text {aux }}\right|_{\Sigma_{\Psi}}=\ldots+\sum_{\substack{k=0 \\
k \text { even }}}^{L-1} \sum_{s=k+1}^{L} \bar{\pi}_{s \alpha_{s}}^{k} \rho_{s \beta_{s}}^{k \alpha_{s}} \pi_{s}^{k+1 \beta_{s}} \\
+\frac{1}{2} \sum_{\substack{k=0 \\
k \text { even }}}^{L} \bar{\pi}_{k \alpha_{k}}^{k} \sigma_{k}^{k \alpha_{k} \beta_{k}} \bar{\pi}_{k \beta_{k}}^{k}+\ldots
\end{gathered}
$$

thereby allowing a gaussian average procedure for gauge invariances at all levels.

In some instances, in lieu of Eq.66.1), one might want to eliminate certain fields rather than antifields. The simplest way to accomplish this is to first perform a 
canonical transformation that interchanges some fields for antifields [104, 230, 259, 44, 252, 257. Then, when Eq. 6.1) is used, elimination of certain antifields will correspond to the elimination of some original fields. Given the freedom to first perform canonical transformations, Eq.(6.1) is quite general.

\subsection{Gauge-Fixed Classical BRST Symmetry}

The gauge-fixed theory inherits a remnant of the original BRST symmetry $\delta_{B}$ in Eq.(4.32). Given $\Psi$, one defines the gauge-fixed BRST operator $\delta_{B_{\Psi}}$ by [24]

$$
\left.\delta_{B_{\Psi}} X \equiv(X, S)\right|_{\Sigma_{\Psi}} \quad,
$$

where $X$ is any functional of the $\Phi^{A}$. For $X=\Phi^{A}$, one obtains

$$
\delta_{B_{\Psi}} \Phi^{A}=\left.\frac{\partial_{l} S}{\partial \Phi_{A}^{*}}\right|_{\Sigma_{\Psi}}
$$

This is the same result as first performing the non-gauge-fixed BRST transformation in Eq.(4.32) and then imposing the gauge-fixing fermion condition in Eq.(6.1). The BRST transformation is a global symmetry of the classical gauge-fixed action since

$$
\begin{gathered}
\delta_{B_{\Psi}} S_{\Psi}=\left.\left(S_{\Psi}, S\right)\right|_{\Sigma_{\Psi}}=\left.\frac{\partial_{r} S_{\Psi}}{\partial \Phi^{A}} \frac{\partial_{l} S}{\partial \Phi_{A}^{*}}\right|_{\Sigma_{\Psi}}= \\
\left.\left(\frac{\partial_{r} S}{\partial \Phi^{A}} \frac{\partial_{l} S}{\partial \Phi_{A}^{*}}\right)\right|_{\Sigma_{\Psi}}+\left.\frac{\partial_{r} S}{\partial \Phi_{B}^{*}} \frac{\partial_{r} \partial_{r} \Psi}{\partial \Phi^{A} \partial \Phi^{B}} \frac{\partial_{l} S}{\partial \Phi_{A}^{*}}\right|_{\Sigma_{\Psi}}=0,
\end{gathered}
$$

where the first term vanishes as a consequence of the classical master equation and the second term vanishes due to statistical symmetry properties of the factors.

Due to the elimination of antifields, $\delta_{B_{\Psi}}$ is not necessarily off-shell nilpotent. For example, consider

$$
\delta_{B_{\Psi}}^{2} \Phi^{A}=\left.\frac{\partial_{r} \partial_{l} S}{\partial \Phi^{C} \partial \Phi_{A}^{*}} \frac{\partial_{l} S}{\partial \Phi_{C}^{*}}\right|_{\Sigma_{\Psi}}+\left.\frac{\partial_{r} \partial_{l} S}{\partial \Phi_{C}^{*} \partial \Phi_{A}^{*}} \frac{\partial_{r} \partial_{l} \Psi}{\partial \Phi^{D} \partial \Phi^{C}} \frac{\partial_{l} S}{\partial \Phi_{D}^{*}}\right|_{\Sigma_{\Psi}},
$$

where the second term arises from the chain rule (see Eq. (A.7) of Appendix A) via the implicit dependence on $\Phi^{D}$ through $\Phi_{C}^{*}$, which is now a functional of the $\Phi$ via Eq.(6.1). By using the identity $0=\frac{\partial_{l}(S, S)}{\partial \Phi_{A}^{*}}$, the first term can be rearranged and then the two terms combine if one notes that

$$
\frac{\partial_{r}\left(S_{\Psi}\right)}{\partial \Phi^{C}}=\left.\frac{\partial_{r} S}{\partial \Phi^{C}}\right|_{\Sigma_{\Psi}}+\left.\frac{\partial_{r} S}{\partial \Phi_{D}^{*}} \frac{\partial_{r} \partial_{l} \Psi}{\partial \Phi^{C} \partial \Phi^{D}}\right|_{\Sigma_{\Psi}} .
$$


The final result is [29, 30, 251]

$$
\delta_{B_{\Psi}}^{2} \Phi^{A}=\left.(-1)^{\epsilon_{A}} \frac{\partial_{r}\left(S_{\Psi}\right)}{\partial \Phi^{C}} \frac{\partial_{l} \partial_{l} S}{\partial \Phi_{C}^{*} \partial \Phi_{A}^{*}}\right|_{\Sigma_{\Psi}} .
$$

The equations of motion for the gauge-fixed action are $\frac{\partial_{r}\left(S_{\Psi}\right)}{\partial \Phi^{C}}=0$. Hence, the gaugefixed BRST symmetry is on-shell nilpotent. From Eq.(4.29), one sees that a necessary condition for off-shell nilpotency is that the algebra is closed, $E_{\alpha \beta}^{j i}=0$, and that there is off-shell reducibility, $V_{a}^{j i}=0$.

Another way of obtaining the on-shell nilpotency is to note that the gauge-fixed BRST transformation agrees with the ordinary BRST transformation evaluated at $\Sigma_{\Psi}$, up to equations of motion. For functionals which do not depend on antifields this follows from Eq.(6.79). For functionals, which depended on antifields before gauge-fixing but are now evaluated at $\Sigma_{\Psi}$, the result follows because

$$
\delta_{B_{\Psi}} \Phi_{A}^{*}(\Phi)=\left.\left(\delta_{B} \Phi_{A}^{*}\right)\right|_{\Sigma_{\Psi}}+\frac{\partial_{l}\left(S_{\Psi}\right)}{\partial \Phi^{A}}
$$

The last term is a gauge-fixed equation of motion. In other words, the processes of performing the off-shell BRST transformation in Eq.4.32) and gauge-fixing via Eq.(6.1) commute up to equations of motion.

\subsection{The Gauge-Fixed Basis}

Another way to gauge-fix the theory is to first perform a canonical transformation to the "gauge-fixed" basis and then set antifields to zero [230, 231, 259, 44, 252]. The canonical transformation in Eq.(4.13) is done with $F_{2}=\Phi^{A} \tilde{\Phi}_{A}^{*}+\Psi(\Phi)$ to give

$$
\Phi^{A}=\tilde{\Phi}^{A}, \quad \Phi_{A}^{*}=\tilde{\Phi}_{A}^{*}+\frac{\partial \Psi}{\partial \Phi^{A}}
$$

so that $\Phi^{A} \rightarrow \Phi^{A}$ and $\Phi_{A}^{*} \rightarrow \Phi_{A}^{*}+\frac{\partial \Psi}{\partial \Phi^{A}}$. The gauge-fixed basis consists of the new tilde fields and antifields.

In this new basis, the original gauge invariances are replaced by the classical gauge-fixed BRST symmetry $\delta_{B_{\Psi}}$. It emerges as the symmetry of the gauge-fixed action $S_{\Psi}$. To understand how this comes about, expand the original proper solution $S$ in a power series in antifields via

$$
S\left[\Phi, \Phi^{*}\right]=S_{0}\left[\phi^{i}\right]+\Phi_{A}^{*} R^{A}+\frac{1}{2} \Phi_{A}^{*} \Phi_{B}^{*} R^{B A}+\ldots,
$$

and perform the change of variables in Eq. 6.85) to obtain

$$
S\left[\Phi, \Phi^{*}\right]=S_{0}\left[\phi^{i}\right]+\frac{\partial \Psi}{\partial \Phi^{A}} R^{A}+\frac{1}{2} \frac{\partial \Psi}{\partial \Phi^{A}} \frac{\partial \Psi}{\partial \Phi^{B}} R^{B A}+\ldots
$$




$$
\begin{gathered}
+\tilde{\Phi}_{A}^{*}\left(R^{A}+\frac{\partial \Psi}{\partial \Phi^{B}} R^{B A}+\ldots\right) \\
+\frac{1}{2} \tilde{\Phi}_{A}^{*} \tilde{\Phi}_{B}^{*}\left(R^{B A}+\ldots\right)+\ldots \\
\equiv \tilde{S}_{0}[\Phi]+\tilde{\Phi}_{A}^{*} \tilde{R}^{A}+\frac{1}{2} \tilde{\Phi}_{A}^{*} \tilde{\Phi}_{B}^{*} \tilde{R}^{B A}+\ldots \equiv \tilde{S}\left[\Phi, \tilde{\Phi}^{*}\right] .
\end{gathered}
$$

The terms in the $\tilde{\Phi}^{*}$ expansion have an interpretation. The $\tilde{\Phi}^{*}$-independent term

$$
\tilde{S}_{0}[\Phi]=\left.S\right|_{\Sigma_{\Psi}} \equiv S_{\Psi}
$$

is the gauge-fixed action. The term linear in $\tilde{\Phi}^{*}$

$$
\tilde{R}^{A}=\left.\frac{\partial_{l} \tilde{S}}{\partial \tilde{\Phi}_{A}^{*}}\right|_{\left\{\tilde{\Phi}_{B}^{*}=0\right\}}=\left.\frac{\partial_{l} S}{\partial \Phi_{A}^{*}}\right|_{\Sigma_{\Psi}} \equiv \delta_{B_{\Psi}} \Phi^{A}
$$

is the generator of gauge-fixed BRST transformations since $\delta_{B_{\Psi}} X=\frac{\partial_{r} X}{\partial \Phi^{A}} \tilde{R}^{A}$. Hence, $\tilde{S}$ is a theory in which gauge transformations have been replaced by the gauge-fixed BRST transformation $\delta_{B_{\Psi}}$. As in Sect. 2, one can define tensors associated with the gauge structure. It is necessary to absorb ghost fields $\mathcal{C}$ into their definition, i.e., $\tilde{R}^{A} \equiv \tilde{R}_{B}^{A} \tilde{\mathcal{C}}^{B}, \tilde{E}^{B A} \equiv \frac{1}{2} \tilde{E}_{D C}^{B A} \tilde{\mathcal{C}}^{C} \tilde{\mathcal{C}}^{D}$, etc.. Since $\delta_{B_{\Psi}}^{2}$ is zero only on-shell, the algebra is open and there exists a non-zero tensor field $\tilde{E}^{B A}$. Comparing Eqs.(2.23) and (6.83), one concludes

$$
\tilde{E}^{B A}=\left.(-1)^{\epsilon_{A}} \frac{\partial_{l} \partial_{l} S}{\partial \Phi_{B}^{*} \partial \Phi_{A}^{*}}\right|_{\Sigma_{\Psi}} .
$$

For an open algebra, according to Eq.(4.29), $\tilde{E}^{B A}(-1)^{\epsilon_{A}}$ is the coefficient of the proper solution quadratic in antifields. Hence, $\left.\tilde{R}^{B A} \equiv \frac{\partial_{l} \partial_{l} \tilde{S}}{\partial \tilde{\Phi}_{B}^{*} \tilde{\Phi}_{A}^{*}}\right|_{\left\{\tilde{\Phi}_{B}^{*}=0\right\}}=\left.\frac{\partial_{l} \partial_{l} S}{\partial \Phi_{B}^{*} \Phi_{A}^{*}}\right|_{\Sigma_{\Psi}}$ should be equal to $\tilde{E}^{B A}(-1)^{\epsilon_{A}}$. This is indeed true since it is equivalent to Eq. (6.89). Hence, the coefficient of $\tilde{S}$ which is quadratic in tilde antifields is the tensor $\tilde{E}^{B A}$ associated with the fact that the gauge-fixed BRST transformations form an open algebra.

The classical master equation for $\tilde{S}$ encodes the algebraic structure of the gaugefixed BRST transformation [133. A straightforward computation gives

$$
0=\frac{1}{2}(\tilde{S}, \tilde{S})=\frac{\partial_{r} S_{\Psi}}{\partial \Phi^{A}} \tilde{R}^{A}+\tilde{\Phi}_{B}^{*}\left(\frac{\partial_{r} \tilde{R}^{B}}{\partial \Phi^{A}} \tilde{R}^{A}+\tilde{R}^{B A} \frac{\partial_{r} S_{\Psi}}{\partial \Phi^{A}}\right)+\ldots
$$

Since each coefficient of the antifield expansion must be zero,

$$
0=\frac{\partial_{r} S_{\Psi}}{\partial \Phi^{A}} \tilde{R}^{A}=\delta_{B_{\Psi}} S_{\Psi}
$$


which is the invariance of the gauge-fixed action under $\delta_{B_{\Psi}}$. The vanishing of the second coefficient in Eq.(6.90) leads to the equation

$$
\frac{\partial_{r} \tilde{R}^{B}}{\partial \Phi^{A}} \tilde{R}^{A}=-\tilde{R}^{B A} \frac{\partial_{r} S_{\Psi}}{\partial \Phi^{A}} .
$$

Eq.(6.92) is related to the on-shell nilpotency of $\delta_{B_{\Psi}}$ :

$$
\begin{gathered}
0=\delta_{B_{\Psi}}\left(\delta_{B_{\Psi}} S_{\Psi}\right)=\frac{\partial_{r}}{\partial \Phi^{B}}\left(\frac{\partial_{r} S_{\Psi}}{\partial \Phi^{A}} \tilde{R}^{A}\right) \tilde{R}^{B}= \\
\frac{\partial_{r} S_{\Psi}}{\partial \Phi^{A}} \frac{\partial_{r} \tilde{R}^{A}}{\partial \Phi^{B}} \tilde{R}^{B}+(-1)^{\left(\epsilon_{A}+1\right) \epsilon_{B}} \frac{\partial_{r} \partial_{r} S_{\Psi}}{\partial \Phi^{B} \partial \Phi^{A}} \tilde{R}^{A} \tilde{R}^{B}
\end{gathered}
$$

The second term vanishes by the statistical properties of the factors. The first term vanishes when Eq. (6.92) is used in conjunction with statistical symmetry arguments. Higher order terms in $0=(\tilde{S}, \tilde{S})$ represent other consistency requirements of the gauge-fixed classical BRST transformation.

In addition to the gauge structure, other concepts generalize to the gauge-fixed basis. In the effective action or any other approach for which antifields remain present, antifields are interpreted as the sources for gauge-fixed BRST transformations. See Sect. 8.4 for more discussion. In the gauge-fixed basis, the classical limit in Eq. (4.24) is replaced by the gauge-fixed condition

$$
\left.\tilde{S}\left[\Phi, \tilde{\Phi}^{*}\right]\right|_{\tilde{\Phi}^{*}=0}=S_{\Psi}[\Phi]
$$

of Eq.(6.88). The proper condition in Eqs. 4.22) and (4.23) becomes 259, 44, 252, 133

$$
\left.\operatorname{rank} \frac{\partial_{l} \partial_{r} S_{\Psi}[\Phi]}{\partial \Phi^{A} \partial \Phi^{B}}\right|_{\left\{\frac{\partial_{r} S_{\Psi}}{\partial \Phi^{D}}=0\right\}}=N
$$

Eq.(6.94) ensures that propagators for the $\Phi^{A}$ are defined so that the usual perturbation theory can be developed. 


\section{Gauge-Fixing Examples}

In this subsection, we present some gauge-fixing procedures for the examples considered in Sects. 3 and 5. The first step is to introduce the auxiliary fields and antifields necessary for gauge-fixing, as specified in Sect. 6.2. The second step is to choose an appropriate $\Psi$. Here, there is quite a bit of freedom and we make specific choices. The third step is to eliminate antifields using $\Psi$ and substitute the results into $S$ to obtain the gauge-fixed action $S_{\Psi}$.

The reader interested in doing exercises can try the following. (i) Derive the equations of motion of the gauge-fixed action. (ii) Determine the effect of the gaugefixed BRST transformation $\delta_{B_{\Psi}}$ on the fields. (iii) Check the gauge-fixed BRST invariance of the gauge-fixed action. (iv) Compute $\delta_{B_{\Psi}}^{2}$ and verify that the nonzero terms, when present, vanish if the equations of motion are used. (v) Perform a gauge-fixing procedure with a different gauge-fixing fermion $\Psi$.

\subsection{The Spinless Relativistic Particle}

Since this system is irreducible, one only needs to append one non-trivial pair $\{\overline{\mathcal{C}}, \bar{\pi}\}$. The non-minimal proper solution is

$$
S=\int \mathrm{d} \tau\left\{\frac{1}{2}\left(\frac{\dot{x}^{2}}{e}-e m^{2}\right)+x_{\mu}^{*} \dot{x}^{\mu} \frac{\mathcal{C}}{e}+e^{*} \dot{\mathcal{C}}+\bar{\pi} \overline{\mathcal{C}}^{*}\right\} .
$$

An example of a gauge-fixing fermion is

$$
\Psi=\int \mathrm{d} \tau \overline{\mathcal{C}}(e-1)
$$

which, via Eq.(6.1), leads to

$$
\overline{\mathcal{C}}^{*}=e-1, \quad e^{*}=\overline{\mathcal{C}}, \quad x_{\mu}^{*}=0, \quad \bar{\pi}^{*}=0
$$

The gauge-fixed action is

$$
S_{\Psi}=\int \mathrm{d} \tau\left\{\frac{1}{2}\left(\frac{\dot{x}^{2}}{e}-e m^{2}\right)+\overline{\mathcal{C}} \dot{\mathcal{C}}+\bar{\pi}(e-1)\right\} .
$$

Because the original algebra is closed, the gauge-fixed BRST transformation $\delta_{B_{\Psi}}$, given by

$$
\begin{gathered}
\delta_{B_{\Psi}} e=\dot{\mathcal{C}}, \quad \delta_{B_{\Psi}} x^{\mu}=\frac{\dot{x}^{\mu} \mathcal{C}}{e}, \quad \delta_{B_{\Psi}} \mathcal{C}=0 \\
\delta_{B_{\Psi}} \bar{\pi}=0, \quad \delta_{B_{\Psi}} \overline{\mathcal{C}}=\bar{\pi} \quad
\end{gathered}
$$


satisfies $\delta_{B_{\Psi}} \delta_{B_{\Psi}}=0$ off-shell. After integrating over $\bar{\pi}$, the gauge $e=1$ is implemented and Eq.(7.4) becomes

$$
S_{\Psi} \rightarrow \int \mathrm{d} \tau\left\{\frac{1}{2}\left(\dot{x}^{2}-m^{2}\right)+\overline{\mathcal{C}} \dot{\mathcal{C}}\right\}
$$

Since the equations of motion for $\bar{\pi}$ and $e$ have been used, Eq.(7.6) is no longer invariant under Eq.(7.5). To derive the modified BRST transformations, start with Eq.(7.1), and perform the shift, $\overline{\mathcal{C}}^{*} \rightarrow \overline{\mathcal{C}}^{*}+e-1, e^{*} \rightarrow e^{*}+\overline{\mathcal{C}}$, to the gauge-fixed basis. Then, determine the equation of motion for $\bar{\pi}$ using $\frac{\partial_{r} S_{\Psi}}{\partial e}=0$ and perform another canonical transformation to shift $\bar{\pi}$ by this solution: $\bar{\pi} \rightarrow \bar{\pi}+\frac{1}{2}\left(\dot{x}^{2} / e^{2}+m^{2}\right)$. The gauge-fixed BRST transformations of the transformed action at the saddle point, $e=1, \bar{\pi}=0$, given by

$$
\delta_{\tilde{B}_{\Psi}} x^{\mu}=\dot{x}^{\mu} \mathcal{C}, \quad \delta_{\tilde{B}_{\Psi}} \mathcal{C}=0, \quad \delta_{\tilde{B}_{\Psi}} \overline{\mathcal{C}}=\frac{1}{2}\left(\dot{x}^{2}+m^{2}\right),
$$

constitute a symmetry of the action in Eq.(7.6), as can easily be checked. Because equations of motion have been used, $\delta_{\tilde{B}_{\Psi}}$ is not longer nilpotent off-shell. Indeed, a

computation of $\delta_{\tilde{B}_{\Psi}}^{2}$ reveals that it is, in general, nonzero; it is zero if the equations of motion, $\ddot{x}^{\mu}=0$ and $\dot{\mathcal{C}}=0$ are used.

\section{2 $\quad$ Yang-Mills Theories}

Since this is an irreducible system, it is necessary to introduce one trivial pair $\left\{\overline{\mathcal{C}}_{a}, \bar{\pi}_{a}\right\}$ for each generator index $a$. Frequently, $\bar{\pi}_{a}$ is denoted by $B_{a}$ and we adopt this notation. The non-minimal proper solution is

$$
S=\int \mathrm{d}^{d} x\left\{-\frac{1}{4} F_{\mu \nu}^{a} F_{a}^{\mu \nu}+A_{a \mu}^{*} D_{b}^{\mu a} \mathcal{C}^{b}+\frac{1}{2} \mathcal{C}_{c}^{*} f_{a b}{ }^{c} \mathcal{C}^{b} \mathcal{C}^{a}+B_{a} \overline{\mathcal{C}}^{* a}\right\} .
$$

To illustrate a gaussian-averaging gauge-fixing procedure, we choose

$$
\Psi=\int \mathrm{d}^{d} x \overline{\mathcal{C}}_{a}\left(-\frac{B^{a}}{2 \xi}+\partial^{\mu} A_{\mu}^{a}\right)
$$

where $\xi$ is a parameter. Elimination of antifields via Eq.6.1) gives

$$
\begin{gathered}
\overline{\mathcal{C}}^{* a}=-\frac{B^{a}}{2 \xi}+\partial^{\mu} A_{\mu}^{a}, \quad B_{a}^{*}=-\frac{\overline{\mathcal{C}}_{a}}{2 \xi}, \\
A_{a \mu}^{*}=-\partial_{\mu} \overline{\mathcal{C}}_{a}, \quad \mathcal{C}_{a}^{*}=0 .
\end{gathered}
$$

The gauge-fixed action $S_{\Psi}$ is

$$
S_{\Psi}=\int \mathrm{d}^{d} x\left\{-\frac{1}{4} F_{\mu \nu}^{a} F_{a}^{\mu \nu}-\partial_{\mu} \overline{\mathcal{C}}_{a} D_{b}^{\mu a} \mathcal{C}^{b}-\left(\frac{B_{a}}{2 \xi}-\partial^{\mu} A_{a \mu}\right) B^{a}\right\}
$$


The gauge-fixed BRST transformations are

$$
\begin{gathered}
\delta_{B_{\Psi}} A^{a \mu}=D^{\mu a}{ }_{b} \mathcal{C}^{b} \\
\delta_{B_{\Psi}} \mathcal{C}^{a}=\frac{1}{2} f_{b c}{ }^{a} \mathcal{C}^{c} \mathcal{C}^{b} \\
\delta_{B_{\Psi}} \overline{\mathcal{C}}_{a}=B_{a} \\
\delta_{B_{\Psi}} B_{a}=0
\end{gathered}
$$

The nilpotency of $\delta_{B_{\Psi}}$ holds off-shell because the original gauge algebra is closed. The gaussian integration over $B_{a}$ can be performed for Eq.(7.11) to give

$$
S_{\Psi} \rightarrow \int \mathrm{d}^{d} x\left\{-\frac{1}{4} F_{\mu \nu}^{a} F_{a}^{\mu \nu}-\partial_{\mu} \overline{\mathcal{C}}_{a} D^{\mu a}{ }_{b} \mathcal{C}^{b}+\frac{\xi}{2} \partial^{\mu} A_{\mu}^{a} \partial^{\nu} A_{a \nu}\right\}
$$

which is known as the Yang-Mills action fixed in the $R_{\xi}$ gauge [1]. The case $\xi=1$ is the Feynman gauge. When $\xi \rightarrow \infty$, the $\bar{\pi}_{a}=B_{a}$ dependence in $\Psi$ of Eq.(7.9) disappears and the Landau gauge $\partial^{\mu} A_{\mu}^{a}=0$ is imposed as a delta-function condition. Because the quadratic forms in Eq.(7.13) are non-degenerate, $\Psi$ is an admissible gauge-fixing fermion. Propagators exist and Eq.(7.13) can be used as an action for the Yang-Mills perturbation series [1, 165].

The BRST symmetry of Eq.(7.13) is determined by the procedure described at the end of Sect. 7.1. One performs canonical transformations to the gauge-fixed basis and then to the classical solution for $B^{a}$. The latter is determined by varying the action with respect to $B^{a}$ itself, and leads to the shift $B^{a} \rightarrow B^{a}+\xi \partial^{\mu} A_{\mu}^{a}$. One finds that the BRST symmetry of Eq.(7.13) is given by

$$
\delta_{\tilde{B}_{\Psi}} A^{a \mu}=D^{\mu a}{ }_{b} \mathcal{C}^{b}, \quad \delta_{\tilde{B}_{\Psi}} \mathcal{C}^{a}=\frac{1}{2} f_{b c}{ }^{a} \mathcal{C}^{c} \mathcal{C}^{b}, \quad \delta_{\tilde{B}_{\Psi}} \overline{\mathcal{C}}^{a}=\xi \partial^{\mu} A_{\mu}^{a}
$$

The gauge-fixed BRST generator $\delta_{\tilde{B}_{\Psi}}$ is only nilpotent on-shell.

\subsection{Topological Yang-Mills Theory}

By using a redundant set of gauge transformations, we have rendered this theory first-stage reducible. The minimal-field proper solution is given in Eq.(5.12). The gauge parameters are $\Lambda^{a}$ and $\varepsilon^{a \mu}$. The number of level 1 gauge invariances is the rank of the group. Correspondingly, we need to introduce the trivial pairs $\left\{\overline{\mathcal{C}}_{a}, \bar{\pi}_{a}\right\}$ and $\left\{\overline{\mathcal{C}}_{a \mu}, \bar{\pi}_{a \mu}\right\}$ at level 0 , and $\left\{\mathcal{C}_{1}^{1 a}, \pi_{1}^{1 a}\right\}$ and $\left\{\overline{\mathcal{C}}_{1 a}, \bar{\pi}_{1 a}\right\}$ at level 1. In keeping with the notation of Sects. 3.3 and 5.3, we use $\eta$ instead of $\mathcal{C}$ for level 1 ghosts: $\overline{\mathcal{C}}_{1 a} \rightarrow \bar{\eta}_{a}$ and $\mathcal{C}_{1}^{1 a} \rightarrow \eta^{1 a}$. We also use $B$ instead of $\bar{\pi}$ at level $0: \bar{\pi}_{a} \rightarrow B_{a}$ and $\bar{\pi}_{a \mu} \rightarrow B_{a \mu}$, and $\Lambda$ instead of $\pi$ at level 1: $\bar{\pi}_{1 a} \rightarrow \bar{\Lambda}_{a}$ and $\pi_{1}^{1 a} \rightarrow \Lambda^{1 a}$. 
To the minimal proper solution in Eq.(5.12), we add

$$
S_{\mathrm{aux}}=\int \mathrm{d}^{4} x\left(B_{a} \overline{\mathcal{C}}^{* a}+B_{a \mu} \overline{\mathcal{C}}^{* a \mu}+\eta_{a}^{1 *} \Lambda^{1 a}+\bar{\Lambda}_{a} \bar{\eta}^{* a}\right) .
$$

For the gauge-fixing fermion, we select

$$
\Psi=\int \mathrm{d}^{4} x\left(\overline{\mathcal{C}}_{a} \partial^{\mu} A_{\mu}^{a}+\overline{\mathcal{C}}_{a \mu} A^{a \mu}+\bar{\eta}_{a} \mathcal{C}^{a}+\eta^{1 a} \overline{\mathcal{C}}_{a}\right)
$$

The elimination of antifields using $\Psi$ gives

$$
\begin{gathered}
\overline{\mathcal{C}}^{* a}=\partial^{\mu} A_{\mu}^{a}+\eta^{1 a}, \quad \overline{\mathcal{C}}^{* a \mu}=A^{a \mu}, \\
\eta_{a}^{*}=0, \quad \eta_{a}^{1 *}=\overline{\mathcal{C}}_{a}, \quad \bar{\eta}^{* a}=\mathcal{C}^{a}, \\
A_{a \mu}^{*}=-\partial_{\mu} \overline{\mathcal{C}}_{a}+\overline{\mathcal{C}}_{a \mu}, \quad \mathcal{\mathcal { C }}_{a}^{*}=\bar{\eta}_{a}, \quad \overline{\mathcal{C}}^{a *}=\eta^{1 a} .
\end{gathered}
$$

The gauge-fixed action becomes

$$
\begin{aligned}
S_{\Psi}= & \int \mathrm{d}^{4} x\left\{\frac{1}{4} F_{\mu \nu}^{a}{ }^{*} F^{a \mu \nu}+\left(-\partial_{\mu} \overline{\mathcal{C}}_{a}+\overline{\mathcal{C}}_{a \mu}\right)\left(D^{\mu a}{ }_{b} \mathcal{C}^{b}+\mathcal{C}^{a \mu}\right)\right. \\
+ & \frac{1}{2} \bar{\eta}_{c} f_{a b}{ }^{c} \mathcal{C}^{b} \mathcal{C}^{a}+A_{c \mu} f_{a b}{ }^{c} \mathcal{C}^{b \mu} \mathcal{C}^{a}+\left(\bar{\eta}_{b}-A_{a \mu} D^{\mu a}{ }_{b}\right) \eta^{b}+ \\
& \left.B_{a}\left(\partial^{\mu} A_{\mu}^{a}+\eta^{1 a}\right)+B_{a \mu} A^{a \mu}+\overline{\mathcal{C}}_{a} \Lambda^{1 a}+\bar{\Lambda}_{a} \mathcal{C}^{a}\right\} .
\end{aligned}
$$

The integration over $B_{a \mu}$ produces $\delta\left(A^{a \mu}\right)$ in the integrand of the functional integral. This delta function can be used to perform the $A^{a \mu}$ integral. Then the integration over $B_{a}, \Lambda^{1 a}$ and $\bar{\Lambda}_{a}$ produces $\delta\left(\eta^{1 a}\right) \delta\left(\overline{\mathcal{C}}_{a}\right) \delta\left(\mathcal{C}^{a}\right)$ which can be used to do the $\eta^{1 a}, \overline{\mathcal{C}}_{a}$ and $\mathcal{C}^{a}$ integrations. The gauge-fixed action is reduced to

$$
S_{\Psi} \rightarrow \int \mathrm{d}^{4} x\left(\overline{\mathcal{C}}_{a \mu} \mathcal{C}^{a \mu}+\bar{\eta}_{a} \eta^{a}\right)
$$

The integration over $\overline{\mathcal{C}}_{a \mu}$ and $\bar{\eta}_{a}$ leads to $\delta\left(\mathcal{C}^{a \mu}\right) \delta\left(\eta^{a}\right)$ which can be used to do the $\mathcal{C}^{a \mu}$ and $\eta^{a}$ integrals. All the functional integrals over fields have been performed, leaving no local degrees of freedom in the action. Not too surprisingly, the integration over the topological action leads to a trivial lagrangian. The functional integral produces a finite number. It is important that all the terms in $\Psi$ in Eq.(7.16) be present. If the last term $\eta^{1 a} \overline{\mathcal{C}}_{a}$ were dropped from $\Psi$, then the integrations over $\Lambda^{1 a}$ and $\overline{\mathcal{C}}_{a}$ would produce singular contributions. 


\subsection{The Antisymmetric Tensor Field Theory}

Like the previous example, this is a first-stage reducible theory, for which one needs to add one trivial pair $\left\{\overline{\mathcal{C}}_{a \mu}, \bar{\pi}_{a \mu}\right\}$ at level 0 and two trivial pairs $\left\{\mathcal{C}_{1}^{1 a}, \pi_{1}^{1 a}\right\}$ and $\left\{\overline{\mathcal{C}}_{1 a}, \bar{\pi}_{1 a}\right\}$ at level 1 . As in the previous subsections, we use $B$ for $\bar{\pi}$ at level 0 , and use $\eta$ and $\Lambda$ respectively for $\mathcal{C}_{1}$ and $\pi_{1}$ at level 1 . To the minimal-field proper solution, one adds the auxiliary action

$$
S_{\text {aux }}=\int \mathrm{d}^{4} x\left(B_{a \mu} \overline{\mathcal{C}}^{* a \mu}+\bar{\Lambda}_{a} \bar{\eta}^{* a}+\eta_{a}^{1 *} \Lambda^{1 a}\right)
$$

An appropriate gauge-fixing fermion is

$$
\Psi=\int \mathrm{d}^{4} x\left(\frac{1}{2} \overline{\mathcal{C}}_{a \mu} \varepsilon^{\mu \nu \rho \sigma} \partial_{\nu} B_{\rho \sigma}^{a}+\bar{\eta}_{a} \partial^{\mu} \mathcal{C}_{\mu}^{a}+\partial^{\mu} \overline{\mathcal{C}}_{a \mu} \eta^{1 a}\right)
$$

The elimination of antifields using $\Psi$ gives

$$
\begin{gathered}
\overline{\mathcal{C}}^{* a \mu}=\frac{1}{2} \varepsilon^{\mu \nu \rho \sigma} \partial_{\nu} B_{\rho \sigma}^{a}-\partial^{\mu} \eta^{1 a}, \quad \eta_{a}^{*}=0, \quad \bar{\eta}^{* a}=\partial^{\mu} \mathcal{C}_{\mu}^{a}, \quad \eta_{a}^{1 *}=\partial^{\mu} \overline{\mathcal{C}}_{a \mu}, \\
B_{a}^{* \rho \sigma}=\varepsilon^{\rho \sigma \mu \nu} \partial_{\mu} \overline{\mathcal{C}}_{a \nu}, \quad \mathcal{C}_{a}^{* \mu}=-\partial^{\mu} \bar{\eta}_{a}, \quad A_{a \mu}^{*}=0 .
\end{gathered}
$$

The gauge-fixed action is

$$
\begin{gathered}
S_{\Psi}=S_{0}+\int \mathrm{d}^{4} x\left(-\frac{1}{2} \partial_{\nu} \overline{\mathcal{C}}_{a \mu}\left(D_{b}^{\nu a} \mathcal{C}^{\mu b}-D_{b}^{\mu a} \mathcal{C}^{\nu b}\right)\right. \\
-\partial_{\mu} \bar{\eta}_{a} D^{\mu a}{ }_{b} \eta^{b}+\frac{1}{4} \partial_{\mu} \overline{\mathcal{C}}_{c \nu} \partial_{\rho} \overline{\mathcal{C}}_{\sigma}^{b} \varepsilon^{\mu \nu \rho \sigma} f_{a b}{ }^{c} \eta^{a}+ \\
\left.B_{a \mu}\left(\frac{1}{2} \varepsilon^{\mu \nu \rho \sigma} \partial_{\nu} B_{\rho \sigma}^{a}-\partial^{\mu} \eta^{1 a}\right)+\bar{\Lambda}_{a} \partial^{\mu} \mathcal{C}_{\mu}^{a}+\partial^{\mu} \overline{\mathcal{C}}_{a \mu} \Lambda^{1 a}\right)
\end{gathered}
$$

where $S_{0}$ is given in Eq.(3.27). One interesting feature of this example is the appearance of a trilinear ghost term. It originates from the gauge-fixing of the bilinear term in antifields in Eq.(5.14). The fields $B_{a \mu}, \bar{\Lambda}_{a}$ and $\Lambda^{1 a}$ are Lagrange multipliers for the gauge conditions $\frac{1}{2} \varepsilon^{\mu \nu \rho \sigma} \partial_{\nu} B_{\rho \sigma}^{a}-\partial^{\mu} \eta^{1 a}=0, \partial^{\mu} \mathcal{C}_{\mu}^{a}=0$ and $\partial^{\mu} \overline{\mathcal{C}}_{a \mu}=0$. If one wants to perform a gaussian averaging over these conditions, one adds

$$
\Psi_{\pi}=-\frac{1}{2} \int \mathrm{d}^{4} x\left(\frac{\overline{\mathcal{C}}_{a \mu} B^{a \mu}}{\xi}+\frac{\bar{\eta}_{a} \bar{\Lambda}^{a}}{\bar{\xi}}+\frac{\Lambda_{a}^{1} \eta^{1 a}}{\xi^{1}}\right)
$$

to $\Psi$ in Eq.(7.21). Here, $\xi, \bar{\xi}$ and $\xi^{1}$ are constants. One could also replace ordinary derivatives $\partial_{\mu}$ by covariant ones $D_{\mu}$ in Eq.(7.21). Then $D_{\mu}$ replaces $\partial_{\mu}$ in Eqs. (7.22) and (7.23). In this case, $A_{a \mu}^{*} \neq 0$, but since $A_{a \mu}^{*}$ does not enter the proper solution in Eq.(5.14), the gauge-fixed action is as in Eq.(7.23) with $\partial_{\mu} \rightarrow D_{\mu}$. 


\subsection{Open String Field Theory}

Since the open bosonic string is an infinite-reducible theory, one introduces the extraghosts $\mathcal{C}_{s}^{k}$ for $k=1,3,5, \ldots$ and $s \geq k$, and $\overline{\mathcal{C}}_{s}^{k}$ for $k=0,2,4, \ldots$ and $s \geq k$ in the Triangular Field Tableau of Diagram 2 for all levels $s$ :

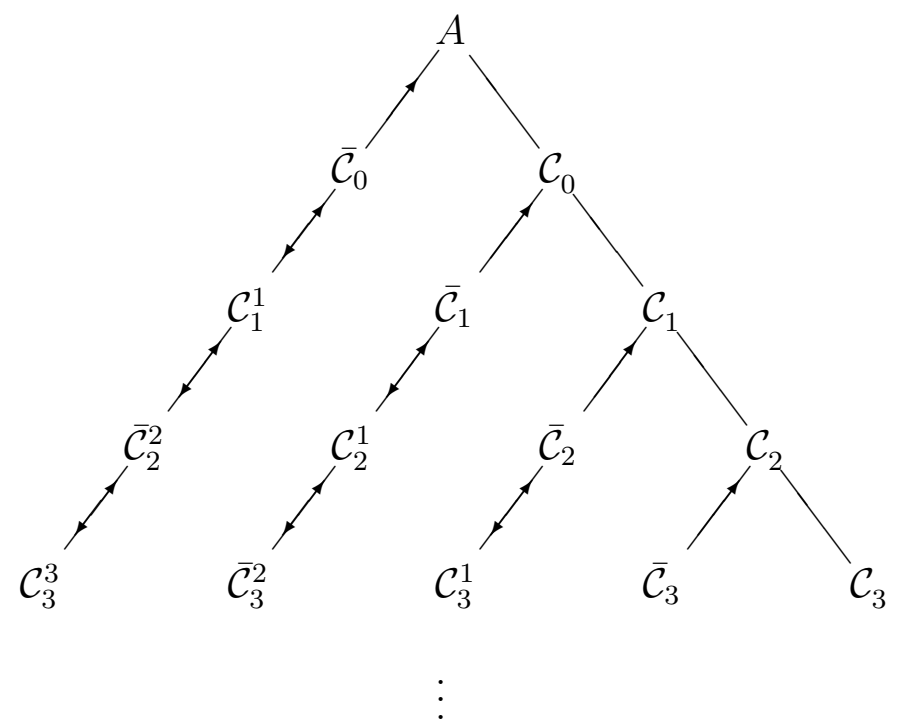

Diagram 9. The Triangular String Field Tableau

In addition, there are the trivial-pair partners $\pi_{s}^{k}$ for $k=1,3,5, \ldots$ and $s \geq k$, and $\bar{\pi}_{s}^{k}$ for $k=0,2,4, \ldots$ and $s \geq k$. Although there are no $\alpha_{i}$ type indices on $\mathcal{C}_{s}^{k}, \overline{\mathcal{C}}_{s}^{k}$, $\pi_{s}^{k}$ and $\bar{\pi}_{s}^{k}$, they are string fields and represent an infinite tower of ordinary particle fields. The ghost numbers of the fields are

$$
\begin{array}{cc}
\operatorname{gh}\left[\mathcal{C}_{s}^{k}\right]=\operatorname{gh}\left[\overline{\mathcal{C}}_{s}^{k *}\right]=s-k, \quad g\left(\mathcal{C}_{s}^{k}\right)=g\left(\overline{\mathcal{C}}_{s}^{k *}\right)=1+k-s, \\
\operatorname{gh}\left[\overline{\mathcal{C}}_{s}^{k}\right]=\operatorname{gh}\left[\mathcal{C}_{s}^{k *}\right]=k-s-1, \quad g\left(\overline{\mathcal{C}}_{s}^{k}\right)=g\left(\mathcal{C}_{s}^{k *}\right)=1+k-s, \\
\operatorname{gh}\left[\bar{\pi}_{s}^{k}\right]=k-s, \quad g\left(\bar{\pi}_{s}^{k}\right)=1+k-s, \\
\operatorname{gh}\left[\pi_{s}^{k}\right]=s-k+1, \quad g\left(\pi_{s}^{k}\right)=1+k-s,
\end{array}
$$

where $\mathrm{gh}[\mathrm{]}$ is the field-antifield ghost number and $g(\mathrm{)}$ is the string ghost number. In Eq.(7.24), we use the abbreviations in Eq.(6.23) for the cases when $k=-1$ and $k=0$. All $\mathcal{C}_{s}^{k}, \overline{\mathcal{C}}_{s}^{k *}$ and $\bar{\pi}_{s}^{k}$ have odd total statistics, while $\overline{\mathcal{C}}_{s}^{k}, \mathcal{C}_{s}^{k *}$ and $\pi_{s}^{k}$ have even total statistics.

The auxiliary action $S_{\text {aux }}$ in Eq.(6.28) is

$$
S_{\mathrm{aux}}=\sum_{\substack{k=0 \\ k \text { even }}}^{\infty} \sum_{s=k}^{\infty} \int{ }^{*} \bar{\pi}_{s}^{k} * \overline{\mathcal{C}}_{s}^{k *}+\sum_{\substack{k=1 \\ k \text { odd }}}^{\infty} \sum_{s=k}^{\infty} \int{ }^{*} \mathcal{C}_{s}^{k *} * \pi_{s}^{k}
$$


where ${ }^{*}$ before a field is the string Hodge star operation. For an arbitrary field or antifield $\varphi, \operatorname{gh}\left[{ }^{*} \varphi\right]=\operatorname{gh}[\varphi]$ but $g\left({ }^{*} \varphi\right)=3-g(\varphi)$. The action in Eq.(7.25) is to be added to the minimal-field proper solution $S$ in Eq.(5.24).

The most convenient gauge for the open bosonic string field theory is the SiegelFeynman gauge 229] which imposes the condition $\bar{c}_{0} A=0$ on the string field $A$. When implemented, the quadratic action of the massless vector $A_{\mu}$ becomes $\frac{1}{2} \int \mathrm{d}^{26} x A_{\mu} \partial_{\nu} \partial^{\nu} A^{\mu}$. Above, $\bar{c}_{0}$ is the zero mode of the antighost of first-quantized open string theory in Sect. 3.8 and Sect. 5.8. It is the conjugate momentum of the zero mode $c_{0}$ of the first-quantized ghost. These modes satisfy

$$
\left\{c_{0}, \bar{c}_{0}\right\}=c_{0} \bar{c}_{0}+\bar{c}_{0} c_{0}=1, \quad c_{0} c_{0}=0, \quad \bar{c}_{0} \bar{c}_{0}=0,
$$

and have string ghost numbers of $g\left(c_{0}\right)=1$ and $g\left(\bar{c}_{0}\right)=-1$. See Sect. 7.7 for more discussion. The gauge fermion $\Psi_{\mathrm{sf}}$, which implements the Siegel-Feynman gauge, has an expansion that, for the first few levels, reads

$$
\begin{gathered}
\Psi_{\mathrm{sf}}=\int{ }^{*} \overline{\mathcal{C}}_{0} * c_{0} \bar{c}_{0} A \\
\int{ }^{*} \overline{\mathcal{C}}_{1} * c_{0} \bar{c}_{0} \mathcal{C}_{0}+\int{ }^{*} \mathcal{C}_{1}^{1} * c_{0} \bar{c}_{0} \overline{\mathcal{C}}_{0}+ \\
\int{ }^{*} \overline{\mathcal{C}}_{2} * c_{0} \bar{c}_{0} \mathcal{C}_{1}+\int{ }^{*} \mathcal{C}_{2}^{1} * c_{0} \bar{c}_{0} \overline{\mathcal{C}}_{1}+\int{ }^{*} \overline{\mathcal{C}}_{2}^{2} * c_{0} \bar{c}_{0} \mathcal{C}_{1}^{1}+\ldots .
\end{gathered}
$$

For all levels,

$$
\Psi_{\mathrm{sf}}=\sum_{\substack{k=0 \\ k \text { even }}}^{\infty} \sum_{s=k}^{\infty} \int * \overline{\mathcal{C}}_{s}^{k} * c_{0} \overline{\mathcal{C}}_{0} \mathcal{C}_{s-1}^{k-1}+\sum_{\substack{k=1 \\ k \text { odd }}}^{\infty} \sum_{s=k}^{\infty} \int{ }^{*} \mathcal{C}_{s}^{k} * c_{0} \bar{c}_{0} \overline{\mathcal{C}}_{s-1}^{k-1}
$$

Since $\Psi_{\mathrm{sf}}$ is not a functional of $\pi_{s}^{k}$ or $\bar{\pi}_{s}^{k}$, a delta-function type gauge-fixing procedure is implemented. Elimination of the antifields via Eq.(6.1) leads to

$$
\begin{aligned}
& \overline{\mathcal{C}}_{s}^{k *}=c_{0} \bar{c}_{0} \mathcal{C}_{s-1}^{k-1}+\bar{c}_{0} c_{0} \mathcal{C}_{s+1}^{k+1}, \quad k=0,2,4, \ldots, \\
& \mathcal{C}_{s}^{k *}=\bar{c}_{0} c_{0} \overline{\mathcal{C}}_{s-1}^{k-1}+c_{0} \bar{c}_{0} \overline{\mathcal{C}}_{s+1}^{k+1}, \quad k=1,3,5, \ldots .
\end{aligned}
$$

When the antifields in Eq.(7.28) are substituted into $S_{\text {aux }}$ of Eq. (7.25) and the integration over $\pi_{s}^{k}$ or $\bar{\pi}_{s}^{k}$ is performed, the following conditions are implemented

$$
\begin{aligned}
& \overline{\mathcal{C}}_{s}^{k *}=0 \Rightarrow \bar{c}_{0} \mathcal{C}_{s-1}^{k-1}=0 \text { and } c_{0} \mathcal{C}_{s+1}^{k+1}=0, \quad k=0,2,4, \ldots, \\
& \mathcal{C}_{s}^{k *}=0 \Rightarrow c_{0} \overline{\mathcal{C}}_{s-1}^{k-1}=0 \text { and } \bar{c}_{0} \overline{\mathcal{C}}_{s+1}^{k+1}=0, \quad k=1,3,5, \ldots,
\end{aligned}
$$

which follow from Eqs.(7.26) and (7.28). For $k \geq 1, \mathcal{C}_{s}^{k}$ and $\overline{\mathcal{C}}_{s}^{k}$ are set equal to zero because any field $\Phi$, for which $\bar{c}_{0} \Phi=0$ and $c_{0} \Phi=0$, must be identically zero, as 
Eq.(7.26) implies. On the other hand, for $\mathcal{C}_{s-1}$ and $\overline{\mathcal{C}}_{s}$, only half these conditions are imposed:

$$
\begin{gathered}
\bar{c}_{0} \mathcal{C}_{s-1}=0, \quad s \geq 0, \\
c_{0} \overline{\mathcal{C}}_{s}=0, \quad s \geq 0 .
\end{gathered}
$$

When these results are used in $\mathcal{C}_{s}^{*} \equiv \mathcal{C}_{s}^{-1 *}=c_{0} \bar{c}_{0} \overline{\mathcal{C}}_{s+1}^{0} \equiv c_{0} \bar{c}_{0} \overline{\mathcal{C}}_{s+1}$, for $s \geq-1$, one finds $\mathcal{C}_{s}^{-1 *}=\overline{\mathcal{C}}_{s+1}$, so that the gauge-fixed string field $\Psi_{\Sigma_{\text {sf }}}$ in Eq. (5.23) is

$$
\Psi_{\Sigma_{\mathrm{sf}}}=\ldots+{ }^{*} \overline{\mathcal{C}}_{s+1}+\ldots+{ }^{*} \overline{\mathcal{C}}_{1}+{ }^{*} \overline{\mathcal{C}}_{0}+A+\mathcal{C}_{0}+\ldots+\mathcal{C}_{s}+\ldots .
$$

The condition $c_{0} \overline{\mathcal{C}}_{s}=0$ implies $\bar{c}_{0}{ }^{*} \overline{\mathcal{C}}_{s}=0$. Hence, the constraints in Eq. (7.30) can be written succinctly as

$$
\bar{c}_{0} \Psi_{\Sigma_{\mathrm{sf}}}=0
$$

Eq.(7.32) implies that $\Psi_{\Sigma_{\text {sf }}}$ has no term proportional to $c_{0}$. Since the string integral is zero unless there is a $c_{0}$ factor present, $Q$ must supply it. Since $Q=c_{0} L_{0}+\ldots$,

$$
Q \rightarrow c_{0} L_{0}
$$

where $L_{0}$ is the string "Klein-Gordon" operator

$$
L_{0}=\frac{1}{2}\left(\partial_{\mu} \partial^{\mu}-\mathcal{M}^{2}\right),
$$

and $\mathcal{M}^{2}$ is the mass-squared operator. The gauge-fixed action $S_{\Psi_{\text {sf }}}$ is

$$
S=\frac{1}{2} \int \Psi_{\Sigma_{\mathrm{sf}}} * c_{0} L_{0} \Psi_{\Sigma_{\mathrm{sf}}}+\frac{1}{3} \int \Psi_{\Sigma_{\mathrm{sf}}} * \Psi_{\Sigma_{\mathrm{sf}}} * \Psi_{\Sigma_{\mathrm{sf}}},
$$

where $\Psi_{\Sigma_{\mathrm{sf}}}$ in Eq.(7.31) satisfies equation (7.32). This is the result obtained in refs. 56 , 246]. Off-shell scattering amplitudes in the Siegel-Feynman gauge have been obtained in refs. 128, 111, 216, 54, 55, 217.

\subsection{The Massless Relativistic Spinning Particle}

This irreducible system has super-reparametrization invariance. It illustrates the gauge-fixing procedure for a simple supergravity theory. The system possesses one ordinary gauge symmetry and one gauge supersymmetry, for which auxiliary trivial pairs $\{\overline{\mathcal{C}}, \bar{\pi}\}$ and $\{\bar{\Gamma}, \bar{\Lambda}\}$ are needed. The fields $\bar{\pi}, \bar{\Gamma}, \overline{\mathcal{C}}^{*}$ and $\bar{\Lambda}^{*}$ are commuting, while $\overline{\mathcal{C}}, \bar{\Lambda}, \bar{\pi}^{*}$ and $\bar{\Gamma}^{*}$ are anticommuting. The auxiliary action for the pairs is

$$
S_{\text {aux }}=\int \mathrm{d} \tau\left(\overline{\overline{\mathcal{C}}} \overline{\mathcal{C}}^{*}+\bar{\Lambda} \bar{\Gamma}^{*}\right)
$$


The fermion

$$
\Psi=\int \mathrm{d} \tau(\overline{\mathcal{C}}(e-1)+\bar{\Gamma} \chi)
$$

imposes the delta-function conditions

$$
e=1, \quad \chi=0
$$

A straightforward calculation shows that the gauge-fixed action becomes

$$
S_{\Psi} \rightarrow \frac{1}{2} \int \mathrm{d} \tau\left(\partial_{\tau} x^{\mu} \partial_{\tau} x_{\mu}-i \psi^{\mu} \partial_{\tau} \psi_{\mu}\right)+\int \mathrm{d} \tau\left(\overline{\mathcal{C}} \partial_{\tau} \mathcal{C}+\bar{\Gamma} \partial_{\tau} \Gamma\right)
$$

For $x^{\mu}$ and $\psi^{\mu}$, this is the flat-space action in Eq.(3.57). Using the superfield formulation in Eq.(3.52) and defining the ghost superfields

$$
G \equiv \mathcal{C}+\theta \Gamma, \quad \bar{G}=\bar{\Gamma}+\theta \overline{\mathcal{C}},
$$

the action in Eq.(7.39) can be written in supersymmetric form as

$$
i \int \mathrm{d} \tau \int \mathrm{d} \theta\left(\frac{1}{2} D_{\theta} X^{\mu} \partial_{\tau} X_{\mu}-D_{\theta} \bar{G} D_{\theta} G\right),
$$

where $D_{\theta}$ is given in Eq.(3.53).

\subsection{The First-Quantized Bosonic String}

The BRST quantization of the first-quantized bosonic string was carried out in refs. [121, 163, 174]. The field-antifield treatment is similar. In the formulation of Sect. 5.8, there are three types of gauge transformations for this irreducible system. Correspondingly, one needs to introduce three level 0 auxiliary trivial pairs $\{\overline{\mathcal{C}}, \bar{\pi}\}$, $\left\{\overline{\mathcal{C}}_{n}, \bar{\pi}_{n}\right\}$, and $\left\{\overline{\mathcal{C}}_{a b}, \bar{\pi}_{a b}\right\}$, where $a, b$ and $n$ take on the values $\sigma$ and $\tau$, and $\overline{\mathcal{C}}_{a b}$ and $\bar{\pi}_{a b}$ are antisymmetric in $a$ and $b$. The auxiliary action $S_{\text {aux }}$ is

$$
S_{\text {aux }}=\int \mathrm{d} \tau \int_{0}^{\pi} \mathrm{d} \sigma\left\{\bar{\pi} \overline{\mathcal{C}}^{*}+\bar{\pi}_{n} \overline{\mathcal{C}}^{* n}+\frac{1}{2} \bar{\pi}_{a b} \overline{\mathcal{C}}^{* a b}\right\} .
$$

This action is to be added to $S$ in Eq.(5.30) to give a total action $S_{\text {total }}=S+S_{\text {aux }}$. For the gauge-fixing fermion, we use

$$
\begin{gathered}
\Psi=\int \mathrm{d} \tau \int_{0}^{\pi} \mathrm{d} \sigma\left\{\overline{\mathcal{C}}_{\tau}\left(e_{\tau}{ }^{\sigma}-e_{\sigma}{ }^{\tau}\right)+\overline{\mathcal{C}}_{\sigma}\left(e_{\tau}^{\tau}-e_{\sigma}{ }^{\sigma}\right)+\right. \\
\left.\overline{\mathcal{C}}_{\tau \sigma}\left(e_{\tau}{ }^{\sigma}+e_{\sigma}{ }^{\tau}\right)+\overline{\mathcal{C}}\left(e_{\tau}{ }^{\tau}+e_{\sigma}{ }^{\sigma}-2 \rho^{-1 / 2}\right)\right\}
\end{gathered}
$$


Such a $\Psi$ leads to the delta-function-type gauge conditions

$$
e_{\tau}^{\tau}=e_{\sigma}^{\sigma}=\rho^{-1 / 2}, \quad e_{\tau}^{\sigma}=e_{\sigma}^{\tau}=0 .
$$

This is known as the conformal gauge. The antifields are

$$
\begin{gathered}
e^{* \tau}=\overline{\mathcal{C}}+\overline{\mathcal{C}}_{\sigma}, \quad e^{* \sigma}=\overline{\mathcal{C}}-\overline{\mathcal{C}}_{\sigma}, \\
e^{* \tau}=\overline{\mathcal{C}}_{\tau \sigma}+\overline{\mathcal{C}}_{\tau}, \quad e^{* \sigma}=\overline{\mathcal{C}}_{\tau \sigma}-\overline{\mathcal{C}}_{\tau}, \\
\overline{\mathcal{C}}^{* \tau}=e_{\tau}^{\sigma}-e_{\sigma}^{\tau}, \quad \overline{\mathcal{C}}^{* \sigma}=e_{\tau}^{\tau}-e_{\sigma}{ }^{\sigma}, \\
\overline{\mathcal{C}}^{* \tau \sigma}=e_{\tau}{ }^{\sigma}+e_{\sigma}{ }^{\tau}, \quad \overline{\mathcal{C}}^{*}=e_{\tau}{ }^{\tau}+e_{\sigma}{ }^{\sigma}-2 \rho^{-1 / 2} \\
X_{\mu}^{*}=0, \quad \mathcal{C}^{*}=\mathcal{C}_{n}^{*}=\mathcal{C}_{a b}^{*}=0 .
\end{gathered}
$$

The gauge-fixed action becomes

$$
\begin{gathered}
S_{\Psi} \rightarrow \int \mathrm{d} \tau \int_{0}^{\pi} \mathrm{d} \sigma\left\{\frac{1}{2} \eta_{\mu \nu}\left(\partial_{\tau} X^{\mu} \partial_{\tau} X^{\nu}-\partial_{\sigma} X^{\mu} \partial_{\sigma} X^{\nu}\right)\right. \\
+\overline{\mathcal{C}}_{\tau} \rho^{-1 / 2}\left(\partial_{\sigma} \mathcal{C}^{\tau}-\partial_{\tau} \mathcal{C}^{\sigma}\right)+\overline{\mathcal{C}}_{\sigma} \rho^{-1 / 2}\left(\partial_{\sigma} \mathcal{C}^{\sigma}-\partial_{\tau} \mathcal{C}^{\tau}\right) \\
+2 \overline{\mathcal{C}}\left(\mathcal{C}^{n} \partial_{n} \rho^{-1 / 2}\right)-\overline{\mathcal{C}}_{\tau \sigma} \rho^{-1 / 2}\left(\partial_{\tau} \mathcal{C}^{\sigma}+\partial_{\sigma} \mathcal{C}^{\tau}+2 \mathcal{C}^{\tau \sigma}\right) \\
\left.+\overline{\mathcal{C}} \rho^{-1 / 2}\left(2 \mathcal{C}-\partial_{\tau} \mathcal{C}^{\tau}-\partial_{\sigma} \mathcal{C}^{\sigma}\right)\right\}
\end{gathered}
$$

where the sum over $n$ is over $\tau$ and $\sigma: \mathcal{C}^{n} \partial_{n} \rho^{-1 / 2}=\mathcal{C}^{\tau} \partial_{\tau} \rho^{-1 / 2}+\mathcal{C}^{\sigma} \partial_{\sigma} \rho^{-1 / 2}$. Integrating over $\mathcal{C}$ and $\mathcal{C}^{\tau \sigma}$ leads to delta functions for $\overline{\mathcal{C}}$ and $\overline{\mathcal{C}}_{\tau \sigma}$, so that the gauge-fixed action becomes the first two lines in Eq.(7.45):

$$
\begin{aligned}
& S_{\Psi} \rightarrow \int \mathrm{d} \tau \int_{0}^{\pi} \mathrm{d} \sigma\left\{\frac{1}{2} \eta_{\mu \nu}\left(\partial_{\tau} X^{\mu} \partial_{\tau} X^{\nu}-\partial_{\sigma} X^{\mu} \partial_{\sigma} X^{\nu}\right)\right. \\
& \left.+\overline{\mathcal{C}}_{\tau} \rho^{-1 / 2}\left(\partial_{\sigma} \mathcal{C}^{\tau}-\partial_{\tau} \mathcal{C}^{\sigma}\right)+\overline{\mathcal{C}}_{\sigma} \rho^{-1 / 2}\left(\partial_{\sigma} \mathcal{C}^{\sigma}-\partial_{\tau} \mathcal{C}^{\tau}\right)\right\} .
\end{aligned}
$$

At this stage, one can either define a new $\overline{\mathcal{C}}_{n}$ field which absorbs the factor of $\rho^{-1 / 2}$ or one can set $\rho(\tau, \sigma)=1$, as long as $D=26$. In either case, since the resulting action is a free theory, it is straightforward to quantize the system. In what follows, we consider the open string case. The equations of motion are

$$
\begin{gathered}
\partial_{\tau} \partial_{\tau} X^{\mu}-\partial_{\sigma} \partial_{\sigma} X^{\mu}=0 \\
\partial_{\tau} \mathcal{C}^{\tau}-\partial_{\sigma} \mathcal{C}^{\sigma}=0, \quad \partial_{\tau} \mathcal{C}^{\sigma}-\partial_{\sigma} \mathcal{C}^{\tau}=0 \\
\partial_{\tau} \overline{\mathcal{C}}_{\tau}-\partial_{\sigma} \overline{\mathcal{C}}_{\sigma}=0, \quad \partial_{\tau} \overline{\mathcal{C}}_{\sigma}-\partial_{\sigma} \overline{\mathcal{C}}_{\tau}=0
\end{gathered}
$$

\footnotetext{
${ }^{6}$ When $D \neq 26$, one cannot do this due to Weyl anomaly. See Sect. 9.3 .
} 
and

$$
\partial_{\sigma} X^{\mu}=\mathcal{C}^{\sigma}=\overline{\mathcal{C}}_{\sigma}=0, \quad \text { at } \sigma=0, \pi,
$$

which follow from a careful treatment of boundary conditions. The solutions are

$$
\begin{aligned}
X^{\mu}(\tau, \sigma) & =x^{\mu}+p^{\mu} \tau+i \sum_{\substack{n=-\infty \\
n \neq 0}}^{\infty} \frac{\alpha_{n}^{\mu}}{n} e^{-i n \tau} \cos (n \sigma) \\
\mathcal{C}^{\tau}(\tau, \sigma) & =\sum_{n=-\infty}^{\infty} c_{n} e^{-i n \tau} \cos (n \sigma) \\
\mathcal{C}^{\sigma}(\tau, \sigma) & =-i \sum_{n=-\infty}^{\infty} c_{n} e^{-i n \tau} \sin (n \sigma) \\
\overline{\mathcal{C}}_{\sigma}(\tau, \sigma) & =-i \sum_{n=-\infty}^{\infty} \bar{c}_{n} e^{-i n \tau} \cos (n \sigma) \\
\overline{\mathcal{C}}_{\tau}(\tau, \sigma) & =\sum_{n=-\infty}^{\infty} \bar{c}_{n} e^{-i n \tau} \sin (n \sigma)
\end{aligned}
$$

where the modes $\alpha_{n}^{\mu}, c_{n}$ and $\bar{c}_{n}(n=-\infty$ to $+\infty, n \neq 0)$ are harmonic-oscillator-like raising and lowering operators satisfying

$$
\begin{gathered}
{\left[p^{\mu}, x^{\nu}\right]=-i \eta^{\mu \nu}, \quad\left[\alpha_{n}^{\mu}, \alpha_{-m}^{\nu}\right]=n \eta^{\mu \nu} \delta_{n m},} \\
\left\{c_{n}, \bar{c}_{-m}\right\}=\delta_{n m}, \quad\left\{c_{n}, c_{m}\right\}=0, \quad\left\{\bar{c}_{n}, \bar{c}_{m}\right\}=0,
\end{gathered}
$$

where $\eta^{\mu \nu}$ is the $D=25+1$ flat space metric with signature $(-,+,+, \ldots)$. The operators $x^{\mu}$ and $p^{\mu}$ correspond to the center-of-mass position and momentum of the string. The zero-mode $c_{0}$ and $\bar{c}_{0}$ play an important role in the Siegel-Feynman gauge of the open bosonic string as discussed in Sect. 7.5. States in the theory are obtained by applying creation operators, corresponding to modes $n$ with $n<0$, to the vacuum states, which are eigenfunctions of $p^{\mu}$ and the zero-mode ghost system.

In second quantization, fields become functionals of the first-quantized variables $X^{\mu}(0, \sigma), \mathcal{C}^{\tau}(0, \sigma)$ and $\overline{\mathcal{C}}_{\sigma}(0, \sigma)$. The string fields for the open bosonic string field theory of Sects. 3.6, 5.6 and 7.5 are such functionals. Alternatively, one may expand the string fields as a linear combination of first-quantized states whose coefficients are ordinary particle fields. For more details, see the reviews in refs. 215, 247].

The gauge-fixed BRST symmetry of the action in Eq.(7.46) is

$$
\begin{gathered}
\delta_{B_{\Psi}} X^{\mu}=\mathcal{C}^{n} \partial_{n} X^{\mu}, \\
\delta_{B_{\Psi}} \mathcal{C}^{m}=-\mathcal{C}^{n} \partial_{n} \mathcal{C}^{m}
\end{gathered}
$$




$$
\begin{gathered}
\delta_{B_{\Psi}} \overline{\mathcal{C}}_{\tau}=-\rho^{1 / 2} \partial_{\tau} X_{\mu} \partial_{\sigma} X^{\mu}+\rho^{1 / 2} \partial_{n}\left(\rho^{-1 / 2} \overline{\mathcal{C}}_{\tau} \mathcal{C}^{n}\right)+\overline{\mathcal{C}}_{\sigma}\left(\partial_{\sigma} \mathcal{C}^{\tau}+\partial_{\tau} \mathcal{C}^{\sigma}\right) \\
\delta_{B_{\Psi}} \overline{\mathcal{C}}_{\sigma}=-\rho^{1 / 2} \frac{1}{2}\left(\partial_{\tau} X_{\mu} \partial_{\tau} X^{\mu}+\partial_{\sigma} X^{\mu} \partial_{\sigma} X_{\mu}\right)+\rho^{1 / 2} \partial_{n}\left(\rho^{-1 / 2} \overline{\mathcal{C}}_{\sigma} \mathcal{C}^{n}\right)+\overline{\mathcal{C}}_{\tau}\left(\partial_{\sigma} \mathcal{C}^{\tau}+\partial_{\tau} \mathcal{C}^{\sigma}\right)
\end{gathered}
$$

where $m$ takes on the values $\tau$ and $\sigma$ and the sum over $n$ is over $\tau$ and $\sigma$. A straightforward computation confirms that

$$
\delta_{B_{\Psi}} S_{\Psi} \equiv \frac{\partial_{r} S_{\Psi}}{\partial \Phi^{A}} \delta_{B_{\Psi}} \Phi^{A}=0
$$

where $S_{\Psi}$ is given in Eq.(7.46). A useful exercise is to perform this computation. Because some fields have been eliminated using equations of motion, $\delta_{B_{\Psi}} \delta_{B_{\Psi}}=0$ only on-shell. 


\section{Quantum Effects and Anomalies}

In this section we discuss some of the quantum aspects of the field-antifield formalism. Basically, the classical quantities and concepts have quantum counterparts: The proper solution $S$ is replaced by a quantum action $W$. The quantum master equation is used in lieu of the classical master equation. The BRST transformation $\delta_{B}$ is generalized to a quantum version $\delta_{\hat{B}}$. It is nilpotent only if the quantum master equation is satisfied. Quantum observables are elements of the cohomology of $\delta_{\hat{B}}$. A violation of the quantum master equation corresponds to a gauge anomaly [251] and is the subject of Sects. 8.5 8.7. In Sects. 8.1 8.4, however, we assume that $W$ satisfies the quantum master equation. Many equations in those subsections are of a formal nature due to the singular operator $\Delta$ in the context of local theories. To obtain a well-defined action of $\Delta$, a regularization scheme must be used. See Sect. 8.7. In Sect. 8.4, the effective action $\Gamma$ is discussed. Antifields become classical and acquire a conceptual interpretation: They are sources for BRST transformations generated by $\Gamma$.

\subsection{Quantum-BRST Transformation and Its Cohomology}

Recall that the condition in Eq.(6.13) that a functional $X$ produces a gaugeinvariant correlation is

$$
(X, W)=i \hbar \Delta X
$$

Introducing the operator $\delta_{\hat{B}} 186$,

$$
\delta_{\hat{B}} X \equiv(X, W)-i \hbar \Delta X
$$

this can be written as $\delta_{\hat{B}} X=0$. The operator $\delta_{\hat{B}}$ is the quantum BRST transformation. It is the quantum generalization of the classical BRST transformation $\delta_{B}$ : As $\hbar$ goes to zero, $\delta_{\hat{B}}$ becomes $\delta_{B}$ in Eq.(4.32). A functional is said to be quantum-BRST invariant if

$$
\delta_{\hat{B}} X=0
$$

Although $\delta_{\hat{B}}$ is nilpotent as a consequence of the quantum master equation

$$
\delta_{\hat{B}} \delta_{\hat{B}} X=-\left(\frac{1}{2}(W, W)-i \hbar \Delta W, X\right)=0
$$

it no longer is a graded derivation since

$$
\delta_{\hat{B}}(X Y)=X\left(\delta_{\hat{B}} Y\right)+(-1)^{\epsilon_{Y}}\left(\delta_{\hat{B}} X\right) Y-i \hbar(-1)^{\epsilon_{Y}}(X, Y)
$$


Suppose $X$ is the BRST transform of another functional $Y$, i.e.,

$$
X=\delta_{\hat{B}} Y=(Y, W)-i \hbar \Delta Y .
$$

Then, one refers to $X$ as quantum-BRST trivial. By the nilpotency of $\delta_{\hat{B}}, X$ is quantum-BRST invariant, but not in an interesting way. In fact, a quantum-BRST trivial operator $X$ produces a zero correlation function, as can be seen as follows. The integrand $\mathcal{I}$ in Eq.(6.6) becomes

$$
\exp \left(\frac{i}{\hbar} W\right) X=-i \hbar \Delta\left(\exp \left(\frac{i}{\hbar} W\right) Y\right) .
$$

Let $\tilde{\mathcal{I}}=-i \hbar \exp \left(\frac{i}{\hbar} W\right) Y$. Then

$$
\begin{gathered}
\left.\int[\mathrm{d} \Phi] \Delta \tilde{\mathcal{I}}\right|_{\Sigma_{\Psi}}=\left.\int[\mathrm{d} \Phi](-1)^{\epsilon_{A}+1}\left(\frac{\partial_{r}}{\partial \Phi^{A}} \frac{\partial_{r}}{\partial \Phi_{A}^{*}} \tilde{\mathcal{I}}\right)\right|_{\Sigma_{\Psi}}= \\
\int[\mathrm{d} \Phi](-1)^{\epsilon_{A}+1} \frac{\partial_{r}}{\partial \Phi^{A}}\left(\left.\left(\frac{\partial_{r}}{\partial \Phi_{A}^{*}} \tilde{\mathcal{I}}\right)\right|_{\Sigma_{\Psi}}\right)+ \\
\left.\int[\mathrm{d} \Phi](-1)^{\epsilon_{A}}\left(\frac{\partial_{r} \partial_{r} \tilde{\mathcal{I}}}{\partial \Phi_{B}^{*} \partial \Phi_{A}^{*}} \frac{\partial_{r} \partial_{r} \Psi}{\partial \Phi^{A} \partial \Phi^{B}}\right)\right|_{\Sigma_{\Psi}} .
\end{gathered}
$$

The first term produces zero since it is a surface term and the second term is zero for symmetry reasons. Hence, an interesting functional $\mathcal{O}$ is one that is non-trivial in the quantum-BRST cohomology, i.e., $\mathcal{O}$ is quantum-BRST invariant but cannot be written as the quantum-BRST transform of some functional:

$$
\delta_{\hat{B}} \mathcal{O}=0 \quad \text { but } \mathcal{O} \neq \delta_{\hat{B}} Y,
$$

for any $Y$. An $\mathcal{O}$ satisfying Eq.(8.5) is called a quantum observable. Observables are considered equivalent if they differ by a quantum-BRST trivial functional.

Unlike the situation at the classical level, quantum observables do not form an algebra. This is due to the fact that $\delta_{\hat{B}}$ is no longer a graded derivation, c.f. Eq. (8.4). This is not surprising since, in the quantum theory, the product of two observables is singular and must be regularized. The regularization process may ruin quantumBRST invariance. However, if the space-time supports of $\mathcal{O}_{1}$ and $\mathcal{O}_{2}$ do not intersect, then $\left(\mathcal{O}_{1}, \mathcal{O}_{2}\right)=0$. In such a case, $\mathcal{O}_{1} \mathcal{O}_{2}$ is quantum-BRST invariant, if $\mathcal{O}_{1}$ and $\mathcal{O}_{2}$ are.

The result obtained above, namely,

$$
\int[\mathrm{d} \Phi] \exp \left(\frac{i}{\hbar} W\right) \delta_{\hat{B}} Y=0,
$$


for various functionals $Y$ produces identities among correlation functions. They embody the Ward identities associated with the BRST invariance of the theory 265, 239, 233, 241.

An example of a correlation function that produces a zero expectation value is

$$
\int[\mathrm{d} \Phi] \exp \left(\frac{i}{\hbar} W_{\Psi}\right)\left(\frac{\partial_{l}\left(\left.X\right|_{\Sigma_{\Psi}}\right)}{\partial \Phi^{A}}+\left.\frac{i}{\hbar} \frac{\partial_{l}\left(W_{\Psi}\right)}{\partial \Phi^{A}} X\right|_{\Sigma_{\Psi}}\right)=0
$$

obtained by integration by parts using Eq.A.5). Up to a normalization factor, Eq.(8.7) is the Schwinger-Dyson equation [94, 221]

$$
\left\langle\frac{\partial_{l}\left(\left.X\right|_{\Sigma_{\Psi}}\right)}{\partial \Phi^{A}}+\left.\frac{i}{\hbar} \frac{\partial_{l}\left(W_{\Psi}\right)}{\partial \Phi^{A}} X\right|_{\Sigma_{\Psi}}\right\rangle=0
$$

In the above, $W_{\Psi}$ denotes the gauge-fixed quantum action

$$
\left.W_{\Psi} \equiv W\right|_{\Sigma_{\Psi}}
$$

and $X$ is any quantum-BRST invariant functional, $\delta_{\hat{B}} X=0$. The derivatives in Eqs. (8.7) and (8.8) involve implicit differentiation of the gauge-fixing fermion, e.g.,

$$
\frac{\partial_{l}\left(\left.X\right|_{\Sigma_{\Psi}}\right)}{\partial \Phi^{A}}=\left.\frac{\partial_{l} X}{\partial \Phi^{A}}\right|_{\Sigma_{\Psi}}+\left.\frac{\partial_{l} \partial \Psi}{\partial \Phi^{A} \partial \Phi^{B}} \frac{\partial_{l} X}{\partial \Phi_{B}^{*}}\right|_{\Sigma_{\Psi}}
$$

and a similar identity holds for $X \rightarrow W$. The fact that the functional in Eq.(8.7) produces a zero expectation suggests that it is quantum-BRST trivial. Indeed, this is the case since

$$
\frac{\partial_{l}\left(\left.X\right|_{\Sigma_{\Psi}}\right)}{\partial \Phi^{A}}+\left.\frac{i}{\hbar} \frac{\partial_{l}\left(W_{\Psi}\right)}{\partial \Phi^{A}} X\right|_{\Sigma_{\Psi}}=\left.\left(\delta_{\hat{B}}\left(\frac{(-1)^{\epsilon(X)}}{i \hbar}\left(\Phi_{A}^{*}-\frac{\partial \Psi}{\partial \Phi^{A}}\right) X\right)\right)\right|_{\Sigma_{\Psi}}
$$

as a short calculation reveals. One obtains the interesting result that the SchwingerDyson equations are a consequence of the quantum-BRST symmetry of the fieldantifield formalism [152]. Reference [2] argued that antifields originate as the antighosts of collective fields that ensure the Schwinger-Dyson equations. This differs from one viewpoint that antifields are the sources of BRST transformations. Equations (8.6) and (8.10) show that the Schwinger-Dyson equations are certain quantum-BRST Ward identities. 


\subsection{Satisfying the Quantum Master Equation}

The quantum master equation

$$
\frac{1}{2}(W, W)-i \hbar \Delta W=0
$$

is the most important requirement of the field-antifield formalism for several reasons: As demonstrated in Sect. 6.1, it guarantees that computations are independent of the gauge-fixing procedure. When the quantum action $W$ is expanded in powers of $\hbar$ via $W=S+O(\hbar)$, the $O\left(\hbar^{0}\right)$ term in Eq. (8.11) is the classical master equation

$$
\frac{1}{2}(S, S)=0
$$

which yields the structure equations. The nilpotency of the quantum BRST operator depends on Eq.(8.11) being satisfied, as can be seen from Eq.(8.3). The quantum cohomology requires the existence of a nilpotent quantum BRST operator. It is used to define the quantum observables of a gauge theory and to determine when two functionals are considered equivalent. In Sect. 8.5, it is argued that the existence of gauge anomalies is related to the violation of the quantum master equation. In short, the field-antifield formalism at the quantum level depends crucially on Eq.(8.11) being satisfied.

The potential difficulty is due to the operator $\Delta$ in Eq.(6.7) which is singular when acting on $S$ or $W$ because usually they are local functionals. Often terms proportional to delta functions and derivatives of delta functions are produced. One therefore needs to regularize $\Delta$. If one can find a regularization such that $(\Delta S)_{\text {reg }}=0$, while maintaining the classical master equation, then one can simply let $W=S . \square$ Then the quantum master equation is satisfied. An example of this situation is a theory without gauge invariances: Antifields are absent in $S$ since the proper solution is given by $S=S_{0}$. Consequently, $\Delta S=0$.

The quantum master equation has a symmetry given by quantum BRST transformations, in which $W \rightarrow W-\varepsilon \delta_{\hat{B}} F$, where $\epsilon(F)=1$ and $\operatorname{gh}[F]=-1$ [27, 103, 152, 75. 253. If Eq.(8.11) is true, and

$$
W=W^{\prime}+\varepsilon\left(W^{\prime}, F\right)+i \hbar \varepsilon \Delta F,
$$

then $W^{\prime}$ also satisfies Eq.(8.11) to order $\varepsilon^{2}$. Eq.(8.13) has a simple interpretation. The term $\varepsilon\left(W^{\prime}, F\right)$ is the change in $W$ due to a canonical transformation (see Eq.(1.12)). The extra term $i \hbar \varepsilon \Delta F$ is due to the non-invariance of the functional integral measure

${ }^{7}$ Strictly speaking, this statement holds for a finite or a regularized theory. Renormalization may require one to add additional terms to $S$. 
under a canonical transformation. The measure effect can be exponentiated and leads to the extra term.

A solution to the quantum master equation ensures that the gauge symmetries survive the process of quantization. Although finding a solution is an important objective, a solution does not necessarily guarantee good behavior of the theory. The theory may still have difficulties in regard to other issues such as locality, unitarity and the presence of Feynman-diagram infinities. These difficulties may be insurmountable or it may be necessary to add new counterterms to achieve the desired properties. There are systems for which the quantum master equation is satisfied but the theory is non-renormalizable (see the next subsection). In short, the quantum theory is not determined solely by the quantum master equation.

\subsection{Remarks on Renormalization}

At the one-loop level and beyond, perturbation theory produces infinities if Feynman-diagram contributions are not regularized. If the regularization involves a cutoff, then standard renormalizability of a field theory means that the cutoff dependence can be absorbed into the coefficients of the terms in the original action [165, 278].8 In this manner, infinities are eliminated. If a regularization procedure can be found that respects the structure of the field-antifield formalism then renormalization is expected to proceed as in the usual field theory case. Infinities should be "absorbable" into the coefficients of the terms in $S$. Regularization methods that seem most convenient for gauge theories, such as the Pauli-Villars scheme, are the best candidates for respecting the field-antifield formalism.

To be concrete, suppose a gauge theory is renormalizable, and suppose the regularization of $\Delta$ happens to give $(\Delta S)_{\text {reg }}=0.9$ Then the $M_{n}$ in Eq. (6.14) can be chosen to be the counterterms removing the cutoff dependence of the theory. The $M_{n}$ involve terms similar to the original action $S$. The quantum master equation in Eq.(6.15) is then satisfied because $\left(\Delta M_{n}\right)_{\text {reg }}=0$ and because $\left(M_{n}, S\right)=\left(M_{n}, M_{m}\right)=0$. These equations hold because of the classical master equation $(S, S)=0$, because $(\Delta S)_{\text {reg }}=0$, because the $M_{n}$ resemble terms in $S$, and because of analyticity of $S$ in coupling constants. If $(\Delta S)_{\text {reg }} \neq 0$ and an infinite number of local counterterms have to be added to the action to satisfy the quantum master equation, then the issue of renormalizability is unclear [ [4]. This situation arises for the closed bosonic string field theory (see Sect. 10.8), as currently formulated.

\footnotetext{
${ }^{8}$ In dealing with renormalizable theories, we also assume that a reasonable gauge-fixing procedure is used, i.e., we exclude procedures leading to interactions that are non-renormalizable by power counting.

${ }^{9}$ A renormalizable theory without gauge invariances is a useful case to keep in mind.
} 
The question of whether the quantum master equation is satisfied and the question of renormalizability are separate issues (although somewhat related). As argued above, a renormalizable theory without gauge invariances is guaranteed to satisfy the quantum master equation. Regularization and renormalization must be performed, even if the quantum master equation is satisfied at all stages of the renormalization process. When gauge invariances are present, the interesting issue is whether the quantum master equation can be maintained after renormalization. A theory can be non-renormalizable but satisfy the quantum master equation: A non-renormalizable theory without gauge invariances is an example. An infinite number of counterterms must be added to the action, but the quantum master equation is satisfied trivially because no antifields enter. In the other extreme, the two-dimensional chiral Schwinger model is anomalous, yet the theory still makes sense and is renormalizable. It is an example of a renormalizable system that does not satisfy the quantum master equation. For more discussion on regularization and renormalization in the field-antifield formalism, which are the natural generalizations of ideas contained in earlier approaches [277, 187, 167], see refs. 2262, 264, 249, 4, 75, 156, 191].

The Zinn-Justin equation [277] has played an important role in analyzing the renormalizability of Yang-Mills theories. The generalization of this equation within the antibracket formalism is the subject of the next section.

\subsection{The Effective Action and the Zinn-Justin Equation}

A useful concept in functional approaches to field theories [1, 165, 278] is the effective action $\Gamma$. When used in the classical approximation, it reproduces computations at the quantum level. This is accomplished by incorporating all loop contributions into effective interactions. These interactions sum the one-particle-irreducible diagrams. Hence, to compute a correlation function in perturbation theory with $\Gamma$, one uses tree diagrams only.

The functional $\Gamma$ is obtained by a Legendre transformation. One performs the functional integral over the quantum fields in the presence of sources. Classical fields are obtained by evaluating the expectation values of the quantum fields in the presence of these source terms. The effective action $\Gamma$ is a functional of the classical fields.

The concept of an effective action also exists in the antibracket formalism [24]. We also denote it by $\Gamma$. It makes the quantum system resemble a classical system by summing loop effects. Since one wants the analog of the "effective antibracket formalism", it is necessary to have classical antifields as well as classical fields. After this is accomplished, one can define an antibracket $(,)_{c}$ in the space of classical fields and antifields. Since computations are performed as though the system is classical, the classical master equation for $\Gamma$ incorporates the quantum master equation of the 
original system.

Consider the functional integral in the presence of sources $J_{A}$, i.e.,

$$
Z\left[J, \Phi_{c}^{*}\right] \equiv \int[\mathrm{d} \Phi] \exp \left[\frac{i}{\hbar}\left(W\left[\Phi, \Phi_{c}^{*}\right]+J_{A} \Phi^{A}\right)\right],
$$

where $J_{A}$ are independent variables that do not depend on $\Phi$ and $\Phi_{c}^{*}$, and have statistics $\epsilon\left(J_{A}\right)=\epsilon\left(\Phi^{A}\right)=\epsilon_{A}$. For reasons that become clear below, we have replaced the $\Phi^{*}$ by classical antifields $\Phi_{c}^{*}$. Actually, to compute perturbatively, one needs to shift the antifields $\Phi_{c A}^{*}$ by $\frac{\partial \Psi}{\partial \Phi^{A}}$, where $\Psi$ is an appropriate gauge-fixing fermion. More precisely, one performs a canonical transformation to the gauge-fixed basis before setting $\Phi_{A}^{*}$ equal to $\Phi_{c A}^{*}$. The net result is that $Z\left[J, \Phi_{c}^{*}+\frac{\partial \Psi}{\partial \Phi}\right]$ is the functional integral of interest. Although a gauge-fixing fermion is present, we omit the dependence on $\Psi$ for notational convenience, in what follows. Since antifields appear in $S$ in the form $S=S_{\Psi}+\Phi_{A}^{*} \delta_{B_{\Psi}} \Phi^{A}+\ldots$, they act as sources for the gaugefixed BRST transformations.

Define a classical field $\Phi_{c}^{A}$ by

$$
\Phi_{c}^{A}\left[J, \Phi_{c}^{*}\right] \equiv \frac{\hbar}{i} \frac{\partial_{l} \ln Z\left[J, \Phi_{c}^{*}\right]}{\partial J_{A}}=\left\langle\Phi^{A}\right\rangle^{J}
$$

where \langle\rangle$^{J}$ denotes an expectation value in the presence of sources:

$$
\langle X\rangle^{J} \equiv \frac{1}{Z\left[J, \Phi_{c}^{*}\right]} \int[\mathrm{d} \Phi] X\left[\Phi, \Phi_{c}^{*}\right] \exp \left[\frac{i}{\hbar}\left(W+J_{A} \Phi^{A}\right)\right] .
$$

Note that $\Phi_{c}^{A}$ is a functional of $J_{B}$ and $\Phi_{c B}^{*}$. In principle, it is possible to invert this relation to determine $J_{A}$ in terms of $\Phi_{c}^{B}$ and $\Phi_{c B}^{*}$. We indicate the solution to this inversion by $J_{c A}: J_{c A}=J_{A}\left[\Phi_{c}, \Phi_{c}^{*}\right]$. The effective action is obtained by a Legendre transformation [165]:

$$
\Gamma\left[\Phi_{c}, \Phi_{c}^{*}\right] \equiv-i \hbar \ln Z_{c}-J_{c A} \Phi_{c}^{A},
$$

where $Z_{c}$ is $Z\left[J, \Phi_{c}^{*}\right]$ evaluated at $J_{A}=J_{c A}$ :

$$
Z_{c} \equiv Z\left[J_{c}, \Phi_{c}^{*}\right]
$$

As a result, $Z_{c}$ is a functional of classical fields and antifields. A straightforward calculation of $\frac{\partial_{r} \Gamma}{\partial \Phi_{c}^{A}}$ gives

$$
\frac{\partial_{r} \Gamma}{\partial \Phi_{c}^{A}}=-J_{c A}-(-1)^{\epsilon_{A} \epsilon_{B}} \frac{\partial_{r} J_{c B}}{\partial \Phi_{c}^{A}} \Phi_{c}^{B}-\left.i \hbar \frac{\partial_{r} \ln Z\left[J, \Phi_{c}^{*}\right]}{\partial J_{B}} \frac{\partial_{r} J_{c B}}{\partial \Phi_{c}^{A}}\right|_{J=J_{c}} .
$$


Using Eq.(8.15), one finds that the last two terms cancel so that

$$
\frac{\partial_{r} \Gamma}{\partial \Phi_{c}^{A}}=-J_{c A}
$$

At this stage, we have made a transition from fields to classical fields. Essentially all quantities are now functionals of $\Phi_{c}^{A}$ and $\Phi_{c A}^{*}$. Given two functionals $X$ and $Y$ of $\Phi_{c}^{A}$ and $\Phi_{c A}^{*}$, define the classical antibracket $(,)_{c}$ by

$$
(X, Y)_{c} \equiv \frac{\partial_{r} X}{\partial \Phi_{c}^{A}} \frac{\partial_{l} Y}{\partial \Phi_{c A}^{*}}-\frac{\partial_{r} X}{\partial \Phi_{c A}^{*}} \frac{\partial_{l} Y}{\partial \Phi_{c}^{A}}
$$

Since the classical antibracket is defined in the same manner as the antibracket, it satisfies the same identities (4.5) - (4.7). Using Eq.(8.19), one obtains

$$
\frac{1}{2}(\Gamma, \Gamma)_{c}=-J_{c A} \frac{\partial_{l} \Gamma}{\partial \Phi_{c A}^{*}}=\left\langle-i \hbar \Delta W+\frac{1}{2}(W, W)\right\rangle_{c},
$$

where the final equality is obtained after some algebra, which makes use of integration by parts. In Eq. (8.21),\langle\rangle$_{c}$ denotes the expectation value in the presence of $J$ but expressed in terms of $\Phi_{c}$ and $\Phi_{c}^{*}$. More precisely, if $X$ is a functional of $\Phi$ and $\Phi^{*}$ then

$$
\left.\langle X\rangle_{c} \equiv\langle X\rangle^{J}\right|_{J=J_{c}}
$$

where \langle\rangle$^{J}$ is defined in Eq.(8.16). Because of the quantum master equation (8.11), Eq.(8.21) becomes

$$
(\Gamma, \Gamma)_{c}=0
$$

a result known as the Zinn-Justin equation [277].

Equation (8.22) allows one to pass from a functional $X$ of the original fields $\Phi$ and $\Phi^{*}$ to a classical functional $\langle X\rangle_{c}$ of classical fields $\Phi_{c}$ and $\Phi_{c}^{*}$ by taking the "classical expectation" of $X: X\left[\Phi, \Phi^{*}\right] \rightarrow\langle X\rangle_{c}$. We refer to $\langle X\rangle_{c}$ as the classical version of the quantum functional $X$. The definition is consistent with the notation for $\Phi_{c}^{A}$ since

$$
\left\langle\Phi^{A}\right\rangle_{c}=\Phi_{c}^{A}
$$

The process $X \rightarrow\langle X\rangle_{c}$ conforms to the idea that a classical variable is the expectation value of the corresponding quantum functional.

Since $\Gamma$ satisfies the Zinn-Justin equation and plays the role of $S$ in the classical antibracket formalism, one can construct by analogy a "classical-quantum" BRST transformation $\delta_{B_{c q}}$ having the same properties as $\delta_{B}$. Define

$$
\delta_{B_{c q}} X \equiv(X, \Gamma)_{c} \quad,
$$


where $X$ is any functional of $\Phi_{c}$ and $\Phi_{c}^{*}$. The nilpotency $\delta_{B_{c q}}^{2}=0$ of $\delta_{B_{c q}}$ follows from the Zinn-Justin equation. Define $X$ to be cq-BRST invariant if $\delta_{B_{c q}} X=0$. According to Eq.(8.23), the effective action $\Gamma$ is cq-BRST invariant since

$$
\delta_{B_{c q}} \Gamma=(\Gamma, \Gamma)_{c}=0
$$

Several of the above-mentioned classical functionals are also cq-BRST invariant. A analysis of $\delta_{B_{c q}} J_{c A}$ reveals that

$$
\delta_{B_{c q}} J_{c A} \equiv\left(J_{c A}, \Gamma\right)_{c}=\frac{1}{2}(-1)^{\epsilon_{A}+1} \frac{\partial_{r}(\Gamma, \Gamma)_{c}}{\partial \Phi_{c}^{A}}=0
$$

Consider

$$
-i \hbar \delta_{B_{c q}} \ln \left(Z_{c}\right)=\left(\Gamma+J_{c A} \Phi_{c}^{A}, \Gamma\right)_{c}=J_{c A}\left(\Phi_{c}^{A}, \Gamma\right)_{c}=J_{c A} \frac{\partial_{l} \Gamma}{\partial \Phi_{c A}^{*}}=0,
$$

where the first equality holds because of Eqs.(8.26) and (8.27), and the last equality follows from Eqs.8.11) and (8.21). Hence,

$$
\delta_{B_{c q}} Z_{c}=\left(Z_{c}, \Gamma\right)_{c}=0 .
$$

The cq-BRST operator $\delta_{B_{c q}}$ is the effective classical version of the quantum-BRST operator $\delta_{\hat{B}} X$. To understand this statement, consider

$$
\begin{gathered}
\delta_{B_{c q}}\left(Z_{c}\langle X\rangle_{c}\right)=\left(Z_{c}\langle X\rangle_{c}, \Gamma\right)_{c}=\frac{\partial_{r}\left(Z_{c}\langle X\rangle_{c}\right)}{\partial \Phi_{c}^{A}} \frac{\partial_{l} \Gamma}{\partial \Phi_{c A}^{*}}-\frac{\partial_{r}\left(Z_{c}\langle X\rangle_{c}\right)}{\partial \Phi_{c A}^{*}} \frac{\partial_{l} \Gamma}{\partial \Phi_{c}^{A}} \\
=\frac{\partial_{r}\left(Z\langle X\rangle^{J}\right)}{\partial J_{c B}}\left(J_{c B}, \Gamma\right)_{c}+(-1)^{\epsilon_{A}} Z_{c}\left\langle\left(\frac{\partial_{r} X}{\partial \Phi_{A}^{*}}+\frac{i}{\hbar} X \frac{\partial_{r} W}{\partial \Phi_{A}^{*}}\right) J_{A}\right\rangle_{c} .
\end{gathered}
$$

The last step follows because \langle\rangle$_{c}$ has dependence on $\Phi_{c}$ and $\Phi_{c}^{*}$ through $J_{c}$. After some algebra which makes use of integration by parts, one finds that

$\delta_{B_{c q}}\left(Z_{c}\langle X\rangle_{c}\right)=Z_{c}\left\langle\delta_{\hat{B}} X+X\left(\Delta W+\frac{i}{2 \hbar}(W, W)\right)\right\rangle_{c}+(-1)^{\epsilon_{B}} Z_{c}\left\langle X \Phi_{c}^{B}\right\rangle\left(J_{c B}, \Gamma\right)_{c}$.

If $\delta_{B_{c q}}\left(\langle X\rangle_{c}\right)$ is computed instead, one obtains the connected part of Eq. (8.29) without a $Z_{c}$ factor. Since Eq.8.27) and the quantum master equation (8.11) are satisfied,

$$
\delta_{B_{c q}}\left(\langle X\rangle_{c}\right)=\left\langle\delta_{\hat{B}} X\right\rangle_{c}
$$

In other words, the cq-BRST variation of the classical version of $X$ is the classical version of the quantum-BRST variation of $X$ [四]. 
The effective action $\Gamma$ in the classical antibracket formalism plays a role analogous to the proper solution $S$ in the ordinary antibracket formalism. Properties obeyed by $\Gamma$ are the same as those obeyed by $S$. Therefore, one can define a BRST structure associated with $\Gamma$ 133. The BRST structure tensors are encoded in $\Gamma$ and the relations among them are given by $(\Gamma, \Gamma)=0$. Expanding in a Taylor series in $\Phi_{c}^{*}$, one has

$$
\Gamma\left[\Phi_{c}, \Phi_{c}^{*}\right]=\Gamma_{0}\left(\Phi_{c}\right)+\Phi_{c A}^{*} \Gamma^{A}\left(\Phi_{c}\right)+\frac{1}{2} \Phi_{c A}^{*} \Phi_{c B}^{*} \Gamma^{B A}\left(\Phi_{c}\right)+\ldots .
$$

Recalling that there is an undisplayed dependence on the gauge-fixing fermion $\Psi$, the above terms have the following interpretation: $\Gamma_{0}\left(\Phi_{c}\right)$ is the one-particle-irreducible generating functional for the basic fields including all loop corrections for the action gauge-fixed using $\Psi$, i.e., $S_{\Psi}$ in Eq. (6.88), and $\Gamma^{A}\left(\Phi_{c}\right)$ is the generator of gauge-fixed cq-BRST transformations. The gauge-fixed cq-BRST operator $\delta_{B_{c q \Psi}}$, the analogy of $\delta_{B_{\Psi}}$, is defined by

$$
\left.\delta_{B_{c q \Psi}} X \equiv(X, \Gamma)\right|_{\Sigma_{\Psi}},
$$

where $X$ is a functional of the $\Phi_{c}^{A}$ only. In the gauge-fixed basis, $\Sigma_{\Psi}$ in Eq. (8.32) means that classical antifields are set to zero. Thus, one has $\delta_{B_{c q \Psi}} \Phi_{c}^{A}=\Gamma^{A}\left(\Phi_{c}\right)$. The tensor $\Gamma^{B A}\left(\Phi_{c}\right)$ is related to the on-shell nilpotency of $\delta_{B_{c q \Psi}}$. In summary, the quantum aspects of the classical theory described by $S$ are reproduced by an effective classical theory governed by $\Gamma$.

\subsection{Quantum Master Equation Violations: Generalities}

Suppose Eq. 8.11) is not zero. Let

$$
\mathcal{A} \equiv \Delta W+\frac{i}{2 \hbar}(W, W)
$$

be the violation of the quantum master equation. A straightforward computation using Eqs.(4.5), (6.8) and (8.1), but not assuming the validity of the quantum master equation, reveals that

$$
\delta_{\hat{B}} \mathcal{A}=0
$$

This equation is consistent with Eqs. (8.21), (8.22) and (8.30) and the Jacobi identity for the antibracket: One has $\frac{1}{2}(\Gamma, \Gamma)_{c}=-i \hbar\langle\mathcal{A}\rangle_{c}$ so that $0=\frac{i}{2 \hbar}\left((\Gamma, \Gamma)_{c}, \Gamma\right)_{c}$ $=\left(\langle\mathcal{A}\rangle_{c}, \Gamma\right)_{c}=\delta_{B_{c q}}\langle\mathcal{A}\rangle_{c}=\left\langle\delta_{\hat{B}} \mathcal{A}\right\rangle_{c}$.

Suppose that

$$
i \mathcal{A}=\delta_{\hat{B}} \Omega+\frac{\hbar}{2}(\Omega, \Omega)=(\Omega, W)-i \hbar \Delta \Omega+\frac{\hbar}{2}(\Omega, \Omega),
$$


where $\Omega$ is a local functional of the fields and antifields. The last term may seem surprising but it is necessary if $\mathcal{A}$ is to satisfy Eq.(8.34): When the quantum master equation is violated as in Eq. (8.33), the nilpotency of the quantum BRST operator no longer holds, as can be seen from Eq. (8.3). The last term in Eq.(8.35) is required to compensate for this effect and ensures Eq.(8.34). Let

$$
W^{\prime}=W+\hbar \Omega
$$

Then, using Eqs.(4.5) and (6.8), one finds that $W^{\prime}$ satisfies the quantum master equation:

$$
\frac{1}{2}\left(W^{\prime}, W^{\prime}\right)-i \hbar \Delta W^{\prime}=0
$$

Since $W^{\prime}$ has the same classical limit as $W$, namely, its order $\hbar^{0}$ term is $S$, one can use $W^{\prime}$ in lieu of $W$ for the quantum action. Then, since the quantum master equation is satisfied, a quantum gauge theory can be defined.

When $\mathcal{A}$ cannot be expressed as in Eq.(8.35) for a local functional $\Omega$, there is an anomaly in the quantum master equation and an obstruction to maintaining gauge symmetries at the quantum level. Since anomalies involve subtleties and singular expressions, they are usually not too easy to compute. The usual approach to this subject uses a loop expansion:

$$
\mathcal{A}=\sum_{l=1}^{\infty} \mathcal{A}_{l} \hbar^{l-1}=\mathcal{A}_{1}+\hbar \mathcal{A}_{2}+\ldots
$$

Using Eqs.(6.14) and (8.37), one finds the following expression for the anomaly at the one-loop level

$$
\mathcal{A}_{1} \equiv \Delta S+i\left(M_{1}, S\right)
$$

To this order, the condition for the absence of an anomaly in Eq.(8.35) is that $\mathcal{A}_{1}$ is a classical BRST variation, i. e.,

$$
\mathcal{A}_{1}=-i\left(\Omega_{1}, S\right)=-i \delta_{B} \Omega_{1}
$$

If $\mathcal{A}_{1}$ can be expressed as in Eq.(8.39) for some local functional $\Omega_{1}$, then, by setting

$$
W^{\prime}=S+\hbar \Omega_{1},
$$

the quantum master equation in Eq.(8.11) is satisfied to order $\hbar$. In words, Eq. (8.39) says that if $\mathcal{A}_{1}$ is expressible as a local BRST variation then effectively there is no one-loop anomaly. Since the second term in Eq.(8.38) is already of this form, the requirement becomes that $\Delta S$ should be a classical-BRST variation of a local functional. 
To order $\hbar$, the equation $\delta_{\hat{B}} \mathcal{A}=0$ in Eq.(8.34) is

$$
\delta_{B} \mathcal{A}_{1}=\left(\mathcal{A}_{1}, S\right)=0 .
$$

In view of Eqs.(8.39) and (8.41), the investigation of anomalies is related to the local BRST cohomology at ghost number one [36, 32, 159, 251, 248]. Although not obvious, it turns out that Eq.(8.41) embodies the Wess-Zumino anomaly consistency equations [268]. In field-antifield formalism, the one-loop master equation anomaly must be classically BRST invariant. If anomalies arise beyond the one-loop level, Eq.(8.34) provides the full quantum consistency conditions: The master equation anomaly must be quantum-BRST invariant.

Note that $\operatorname{gh}[\mathcal{A}]=1$. Of the fields in Eq.(4.1), only $\mathcal{C}_{0}^{\alpha_{0}} \equiv \mathcal{C}^{\alpha}$ has ghost number one. Hence, one may write

$$
i \mathcal{A}=a_{\alpha}(\phi) \mathcal{C}^{\alpha}+\ldots,
$$

where the omitted terms involve antifields. Sometimes these terms are absent so that Eq. (8.42) gives the structure of the quantum-master-equation anomaly. Although not obvious, the coefficients $a_{\alpha}(\phi)$ are the usual gauge anomalies [251]. In other words, a quantum-master-equation anomaly and a gauge anomaly are equivalent.

\subsection{Canonical Transformations and the Quantum Master Equation}

Canonical transformations preserve the quantum master equation as long as $W$ is appropriately transformed [157]. Consider an infinitesimal canonical transformation as in Eq. (4.10) governed by $F$. Normally a functional $G$ transforms as $G \rightarrow G+$ $\varepsilon(G, F)$. For $W$, however, one must add an extra term $i \hbar \varepsilon \Delta F$ to compensate for "measure effects". Hence, the transformation rule for $W$ is taken to be

$$
W \rightarrow W+\varepsilon(W, F)+i \hbar \varepsilon \Delta F+O\left(\varepsilon^{2}\right)=W-\varepsilon \delta_{\hat{B}} F+O\left(\varepsilon^{2}\right) \quad .
$$

According to Eq.(8.13), Eq. 8.43) is a symmetry of the quantum master equation [27, 152, 103, 175, 253]. If $W$ is a solution to the quantum master equation, changing $W$ as in Eq.8.43) will not upset the solution.

The same conclusion holds for finite transformations governed by $F_{2}$ in Eq.(4.13). In this case, one transforms from $\left\{\Phi, \Phi^{*}\right\}$ variables to $\left\{\tilde{\Phi}, \tilde{\Phi}^{*}\right\}$ variables via Eq.(4.13). The transformation rule for $W$ is [27]

$$
\widetilde{W}\left[\tilde{\Phi}, \tilde{\Phi}^{*}\right]=W\left[\Phi, \Phi^{*}\right]-\frac{i \hbar}{2} \ln J,
$$


where the jacobian factor $J$ is the berezinian governing the change from $\left\{\Phi, \Phi^{*}\right\}$ variables to tilde variables. Then, $\widetilde{W}$ satisfies the quantum master equation exactly in the tilde variables if $W$ satisfies it in the $\left\{\Phi, \Phi^{*}\right\}$ variables. A detailed proof of this result and a formula for $J$ can be found in ref. 251]. Here, we provide a few key steps. Define $\widetilde{\Delta}$ as the analog of $\Delta$ in the transformed variables, i.e.,

$$
\widetilde{\Delta} \equiv(-1)^{\epsilon_{A}+1} \frac{\partial_{r}}{\partial \tilde{\Phi}^{A}} \frac{\partial_{r}}{\partial \tilde{\Phi}_{A}^{*}} .
$$

If $G$ is an arbitrary functions of $\tilde{\Phi}$ and $\tilde{\Phi}^{*}$, then

$$
\widetilde{\Delta} G\left[\tilde{\Phi}, \tilde{\Phi}^{*}\right]=\Delta G\left[\tilde{\Phi}\left[\Phi, \Phi^{*}\right], \tilde{\Phi}^{*}\left[\Phi, \Phi^{*}\right]\right]-\frac{1}{2}(G, \ln J) .
$$

When $\Delta$ acts on $G$ on the right-hand side of Eq.(8.45), the tilde fields should be regarded as functionals of $\Phi$ and $\Phi^{*}$, as indicated. The chain rule for derivatives is then used. This produces $\widetilde{\Delta} G$ plus an extra term, which is equal to $\frac{1}{2}(G, \ln J)$ and needs to be subtracted to obtain the identity in Eq.(8.45). Using Eqs. (8.44) and (8.45), one finds,

$$
\begin{gathered}
i \hbar \widetilde{\Delta} \widetilde{W}-\frac{1}{2}(\widetilde{W}, \widetilde{W})=i \hbar\left\{\Delta \widetilde{W}-\frac{1}{2}(\widetilde{W}, \ln J)\right\}-\frac{1}{2}(\widetilde{W}, \widetilde{W}) \\
=i \hbar\left\{\Delta W-\frac{i \hbar}{2} \Delta \ln J-\frac{1}{2}\left(W-\frac{i \hbar}{2} \ln J, \ln J\right)\right\}-\frac{1}{2}\left(W-\frac{i \hbar}{2} \ln J, W-\frac{i \hbar}{2} \ln J\right) \\
=i \hbar \Delta W-\frac{1}{2}(W, W)+\frac{\hbar^{2}}{2}\left\{\Delta \ln J-\frac{1}{4}(\ln J, \ln J)\right\} .
\end{gathered}
$$

It can be shown [27, 251] that $\Delta \ln J=\frac{1}{4}(\ln J, \ln J)$, so that the last term is zero. Hence, if $W$ satisfies the quantum master equation, then $\widetilde{W}$ satisfies the tilde version of the quantum master equation. For infinitesimal transformations,

$$
\begin{gathered}
\tilde{\Phi}^{A}=\Phi^{A}-\varepsilon\left(\Phi^{A}, F\right)+O\left(\varepsilon^{2}\right), \\
\tilde{\Phi}_{A}^{*}=\Phi_{A}^{*}-\varepsilon\left(\Phi_{A}^{*}, F\right)+O\left(\varepsilon^{2}\right), \\
J=1-2 \varepsilon \Delta F+O\left(\varepsilon^{2}\right) .
\end{gathered}
$$

Then,

$$
\widetilde{W}\left[\Phi, \Phi^{*}\right]=W\left[\Phi, \Phi^{*}\right]+\varepsilon(W, F)+i \hbar \Delta F+O\left(\varepsilon^{2}\right),
$$

so that

$$
W\left[\Phi, \Phi^{*}\right] \rightarrow \widetilde{W}\left[\Phi, \Phi^{*}\right]=W-\varepsilon \delta_{\hat{B}} F+O\left(\varepsilon^{2}\right),
$$

and one recovers Eq.(8.43). The identity $\Delta \ln J-\frac{1}{4}(\ln J, \ln J) \sim O\left(\varepsilon^{2}\right)$ follows from Eq. (8.46) and $\Delta^{2}=0$.

One can take advantage of canonical transformations in analyzing potential anomalies by going to a basis for which the computation is simpler. 


\subsection{The Anomaly at the One-Loop Level}

The quantities that appear in the violation of the quantum master equation in Sect. 8.2 involve both fields and antifields. As a consequence, one must use the action before any elimination of antifields. On the other hand, since propagators are needed to perform perturbative computations, a gauge-fixing procedure is required. Both these requirements can be satisfied by working in the gauge-fixed basis described in Sect. 6.6. It is achieved by performing a canonical transformation with the gaugefixing fermion $\Psi$ so that $\Phi_{A}^{*} \rightarrow \Phi_{A}^{*}+\frac{\partial \Psi}{\partial \Phi^{A}}$. Throughout the rest of this section, we assume that an admissible $\Psi$ has been selected and that the shift to the gauge-fixed basis has been performed. According to the result in Sect. 8.6, if the quantum master equation is satisfied and a canonical transformation is performed to a new basis then, by appropriately adjusting the action, the quantum master equation is satisfied in the new basis. Hence, the existence or non-existence of an anomaly is independent of the choice of basis, although the form of the anomaly may depend on this choice.

There are different ways of obtaining the anomaly. We mostly follow the approach of ref. 251 and briefly mention other methods at the end of this subsection. Reference [251] obtained general formulas for the antifield-independent part of the one-loop anomaly using a Pauli-Villars regularization scheme. Since the derivation is somewhat technical, we present only the final results. For more details, see refs. 87, 251, 259, 78, 75, 252, 257, 253. In particular, refs. 75, 253 have an extensive discussion of Pauli-Villars regularization in the antibracket formalism to which we refer the reader.

The goal of the next few paragraphs is to obtain a regularized expression for $\Delta S$, denoted by $(\Delta S)_{\text {reg. }}$. The anomaly $\mathcal{A}_{1}$ is essentially $(\Delta S)_{\text {reg }}$ since the $M_{1}$ term in Eq. (8.38) is eliminated as a possible violation of the quantum master equation via Eq.(8.40) with $\Omega_{1}=-M_{1}$, i.e., the counterterm $-\hbar M_{1}$ is added to the action. Define

$$
\begin{aligned}
K_{B}^{A} & \equiv \frac{\partial_{l}}{\partial \Phi_{A}^{*}} \frac{\partial_{r}}{\partial \Phi^{B}} S\left[\Phi, \Phi^{*}\right] \\
Q_{A B} & \equiv \frac{\partial_{l}}{\partial \Phi^{A}} \frac{\partial_{r}}{\partial \Phi^{B}} S\left[\Phi, \Phi^{*}\right]
\end{aligned}
$$

Note that $Q$ involves derivatives with respect to fields and not antifields. If expanded about a stationary point, $Q$ becomes the quadratic form for the fields. In such an expansion, the inverse of $Q$ is the propagator. The properness condition in the gaugefixed basis guarantees that propagators exist. An operator $\mathcal{O}$ used to regulate $\Delta S$ is related to $Q$ by

$$
\mathcal{O}^{A}{ }_{B} \equiv\left(T^{-1}\right)^{A C} Q_{C B}
$$

where $T_{A C}$ is an arbitrary invertible matrix satisfying $T_{B A}=(-1)^{\epsilon_{A}+\epsilon_{B}+\epsilon_{A} \epsilon_{B}} T_{A B}$. The inverse of $T$ obeys $\left(T^{-1}\right)^{B A}=(-1)^{\epsilon_{A} \epsilon_{B}}\left(T^{-1}\right)^{A B}$. The Grassmann statistics of 
$T_{A B}$ and $\left(T^{-1}\right)^{A B}$ are $\epsilon_{A}+\epsilon_{B}(\bmod 2)$. Eq.(8.49) implies $Q_{A B}=T_{A C} \mathcal{O}^{C}{ }_{B}$. In the regularization scheme of ref. 2251], the matrix $T$ appears in the mass term for the regulating Pauli-Villars fields. In that approach, the violation of the quantum master equation is shifted from the $\Delta$ term to the $(S, S)$ term.

The regulated expression for $(\Delta S)_{\text {reg }}$ is [251]

$$
(\Delta S)_{\mathrm{reg}}=\left[F_{B}^{A}\left(\frac{1}{1-\frac{\mathcal{O}}{M}}\right)^{B}\right]_{0},
$$

where

$$
F_{C}^{A} \equiv K^{A}{ }_{C}+\frac{1}{2}\left(T^{-1}\right)^{A D}\left(\delta_{B} T\right)_{D C}(-1)^{\epsilon_{C}},
$$

and where $\delta_{B} T$ denotes the classical BRST transform of $T: \delta_{B} T=(T, S)$. In Eq. (8.50), the sum over $A$ and $B$, leading to the trace, involves the quadratic form of fields only and not that of antifields. The subscript 0 on the square brackets in Eq.(8.50) indicates that the term independent of $M$ is to be extracted. Here, $M$ denotes a regulator mass. The one-loop nature of Eq. (8.50) is evident by the presence of the propagator factor $-i /(M-\mathcal{O}) \rightarrow(i M)^{-1} /(1-\mathcal{O} / M)$ and the sum over the index $A$ indicating a trace.

When $\mathcal{O}$ is quadratic in space-time derivatives, one lets

$$
\mathcal{R}=\mathcal{O} \quad, \quad \mathcal{M}^{2}=M,
$$

where $\mathcal{R}$ denotes the quadratic regulator operator and $\mathcal{M}$ denotes the regulating mass or cutoff. When $\mathcal{O}$ is linear in space-time derivatives, it is convenient to multiply on the right in the trace in Eq. (8.50) by $1 /(1+\mathcal{O} / M)(1+\mathcal{O} / M)$ and carry out the multiplication of $1 /(1-\mathcal{O} / M)$ with $1 /(1+\mathcal{O} / M)$. Eq.(8.50) can then be manipulated into the form [251]

$$
(\Delta S)_{\mathrm{reg}}=\left[F^{\prime A}{ }_{B}\left(\frac{1}{1-\frac{\mathcal{R}}{\mathcal{M}^{2}}}\right)^{B}\right]_{0},
$$

where

$$
\mathcal{R}=\mathcal{O}^{2} \quad, \quad \mathcal{M}=M
$$

and

$$
F^{\prime A}{ }_{C} \equiv F^{A}{ }_{C}-\frac{1}{2 M}\left(\delta_{B} \mathcal{O}\right)^{A}{ }_{C}(-1)^{\epsilon_{C}}
$$

with $\delta_{B} \mathcal{O}=(\mathcal{O}, S)$.

Summarizing, in the quadratic momenta case, $(\Delta S)_{\text {reg }}$ is given by Eqs. (8.50) and (8.51). This is the same as using Eq.(8.53) with $\mathcal{R}$ and $\mathcal{M}$ given in Eq.(8.52) and 
with $F$ in Eq.(8.51) replacing $F^{\prime}$. In the linear momenta case, Eqs. (8.53)-(8.55) are used.

For the situation in which $\mathcal{O}$ is quadratic in momenta or in the case where $\delta_{B} \mathcal{O}$ does contribute in Eq. (8.53), one can replace $1 /\left(1-\mathcal{R} / \mathcal{M}^{2}\right)$ by $\exp \left(\mathcal{R} / \mathcal{M}^{2}\right)$. This follows by writing $1 /\left(1-\mathcal{R} / \mathcal{M}^{2}\right)=\int_{0}^{\infty} \exp \left[-\lambda\left(1-\mathcal{R} / \mathcal{M}^{2}\right)\right] \mathrm{d} \lambda$, and inserting in Eq.(8.50) or Eq.8.53):

$$
\begin{gathered}
\int_{0}^{\infty} \mathrm{d} \lambda \exp (-\lambda)\left[F^{A}\left(\exp \left(\frac{\lambda \mathcal{R}}{\mathcal{M}^{2}}\right)\right)^{B}\right]_{A}= \\
\int_{0}^{\infty} \mathrm{d} \lambda \exp (-\lambda) \sum_{n=-p}^{\infty} f_{n}\left[\left(\frac{\lambda}{\mathcal{M}^{2}}\right)^{n}\right]_{0}=f_{0}= \\
\sum_{n=-p}^{\infty} f_{n}\left[\left(\frac{1}{\mathcal{M}^{2}}\right)^{n}\right]_{0}=\left[F^{A}{ }_{B}\left(\exp \left(\frac{\mathcal{R}}{\mathcal{M}^{2}}\right)\right)_{A}^{B}\right]_{0},
\end{gathered}
$$

where a Laurent expansion in $\frac{1}{\mathcal{M}^{2}}$ has been performed. The resulting expression,

$$
(\Delta S)_{\mathrm{reg}}=\left[F_{B}^{A}\left(\exp \left(\frac{\mathcal{R}}{\mathcal{M}^{2}}\right)\right)^{B}\right]_{0},
$$

corresponds to the Fujikawa form [120] of the regularization [251] . In the limit $\mathcal{M} \rightarrow \infty$, terms of order $1 / \mathcal{M}^{n}$ for $n>0$ vanish, whereas terms with $n<0$ blow up. The regularization scheme consists of dropping the terms that blow up. In the PauliVillars regularization, this is achieved by adding fields with appropriate statistics, couplings and masses to cancel all $n<0$ terms. As a consequence, only the $n=0$ term remains.

In the quadratic momentum case, Eq.(8.50) can be manipulated into the following supertrace form 133

$$
(\Delta S)_{\mathrm{reg}}=\left[-\frac{1}{2}\left(\mathcal{R}^{-1} \delta \mathcal{R}\right)^{A}{ }_{B}\left(\frac{1}{1-\frac{\mathcal{R}}{\mathcal{M}^{2}}}\right)_{A}^{B}(-1)^{\epsilon_{A}}\right]_{0},
$$

which only involves the quadratic regulator $\mathcal{R}$. Eq. 8.57) shows that if $\delta \mathcal{R}=[\mathcal{R}, G]$ for some $G$ then the anomaly vanishes, as a consequence of the cyclicity of the trace [133. Formally, Eq. (8.57) is

$$
(\Delta S)_{\mathrm{reg}}=\delta_{B}\left[-\frac{1}{2}\left(\ln \left(\frac{-\mathcal{R}}{\mathcal{M}^{2}-\mathcal{R}}\right)\right)_{A}^{A}(-1)^{\epsilon_{A}}\right]_{0},
$$

which is tantamount to demonstrating that $(\Delta S)_{\text {reg }}$ satisfies the one-loop anomaly consistency condition $\delta_{B}(\Delta S)_{\mathrm{reg}}=0$ in Eq.(8.41) since $(\Delta S)_{\text {reg }}$ is a classical BRST 
variation and $\delta_{B}$ is nilpotent. If the non-BRST-invariant part of the quantity corresponding to $[\ldots]_{0}$ in Eq. (8.58) is local then there is no one-loop anomaly according to Eqs. 8.39) and (8.40). The expression in square brackets in Eq. 8.58) turns out to be the one-loop contribution to the effective action. Eq.(8.58) says that the one-loop anomaly is the BRST variation of this one-loop contribution.

In the approach of ref. [251], Eq.(8.50), (8.53), or (8.56) is evaluated using standard perturbation theory about a stationary point. Antifields are finally set to zero and $\mathcal{R}$ is evaluated at $\Sigma$, i.e, on-shell.

Another approach to anomalies, which retains antifields, is developed in refs. 259, 133, 252, 257. At one loop, results agree with the above. It has the advantage of making it easier to compute antifield-dependent terms in the anomaly, if present. Such terms might arise if there is an anomalous non-closure of BRST transformations or some other difficulty with a BRST-structure equation. Antifields may be retained or eliminated at any stage of a computation. A third approach is to use the effective action $\Gamma$ in Eq. (8.21) [32, 159, 248]. In this method, antifields must be retained. Information about anomalies can be obtained using cohomolgical arguments based on the Wess-Zumino consistency conditions [268]. One must compute the coefficients of candidate terms using perturbative methods [89, 57, 99, 8].

Eq.(8.57) demonstrates that fields for which $\delta \mathcal{R}$ is zero, do not contribute to the anomaly. Non-propagating degrees of freedom, such as gauge-fixed fields and delta-function-generating Lagrange multipliers $\pi$, are not expected to contribute [253] because anomalies arise from loop effects. In practice, the evaluation of anomalies is performed perturbatively. Consequently, one expands around a stationary point. In the gauge-fixed basis, this involves expanding about $\Sigma$, that is, around $\Phi_{A}^{*}=0$, if the method retains antifields, 1 and about $\Phi^{A}=\Phi_{0}^{A}$, where the $\Phi_{0}^{A}$ satisfy the gaugefixed equations of motion. One must be careful to compute $F^{\prime A}{ }_{C}$ in Eq.8.55) before expanding about the perturbative saddle point. In a delta-function implementation of gauge-fixing, it is advantageous, at the beginning of a computation, to perform a canonical transformation that shifts the Lagrange multipliers $\pi$ by solutions to equations of motion. These equations are generated by the fields which are being gauge-fixed. Such a canonical transformation ensures that gauge-fixed fields and the $\pi$ do not mix on-shell with the other fields of the system. This is illustrated in the first and third sample computations of Sect. 9 .

The choice of $T$ in Eq. (8.49), which determines the regulator $\mathcal{O}$, is at one's disposal. The requirements on $T$ are that it be invertible and that it lead to a quadratic

\footnotetext{
${ }^{10}$ The BRST-invariant part, which may be non-local, gives zero contribution to the anomaly since $\delta_{B}$ is applied to it.

${ }^{11}$ In methods for which antifields are eliminated, one does not expand about $\Phi_{A}^{*}=0$ but simply sets $\Phi_{A}^{*}=0$.
} 
regulator $\mathcal{R}$ that is negative definite after a Wick rotation to Euclidean space. Modifying $T$ changes the form of the anomaly. In particular, when more than one gauge symmetry is present, varying $T$ changes the coefficients $a_{\alpha}$ in Eq. (8.42). If some nonzero $a_{\alpha}$ are made zero and vice-versa, the anomaly is shifted from being associated with one type of gauge symmetry to another. This is analogous to the well known situation for anomalous chiral gauge theories in four dimensions: The anomaly can be moved from the axial vector sector to the vector sector, if so desired. See, for example, Sect. 4.1 of ref. 251]. Although we do not present any examples of this effect, it is well illustrated in ref. [251]. In performing anomaly calculations, it is useful to choose $T$ to render a computation as simple as possible. For a similar reason, it is also useful to perform certain canonical transformations before commencing a calculation. 


\section{Sample Anomaly Calculations}

In this section, we present computations of $(\Delta S)_{\text {reg }}$ to see whether the quantum master equation is violated at the one-loop level. In general, the analysis is complicated and lengthy. For this reason, we treat only three cases: the spinless relativistic particle, the chiral Schwinger model, and the first-quantized bosonic string. We use the method of ref. 251, which we have outlined in Sect. 8.7. The first step in the procedure is to transform to the gauge-fixed basis. One then has the option of performing additional canonical transformations. They can be used to partially diagonalize the system, so that potential contributions to the anomaly can be calculated separately from various sectors. The second step is to compute the matrices $K^{A}{ }_{B}$ and $Q_{A B}$ in Eqs. 8.47) and (8.48). The third step is to select a $T_{A B}$ matrix so that the operator $\mathcal{O}^{A}{ }_{B}$ in Eq. (8.49) can be obtained. A judicious choice of $T_{A B}$ can simplify a computation. One then obtains $F^{A}{ }_{B}$ from Eq. (8.51) and $\mathcal{R}$ from Eq.(8.52) or Eq.(8.54). The final step is to use the anomaly formula in Eq. (8.56). Standard perturbation theory is performed, in which one expands about a stationary point and sets antifields to zero. For sample computations using a method that retain antifields throughout the computation, see ref. 133, 257. Other useful results for anomaly calculations can be found in 2251, 78, 75, 133, 162, 193, 14, 64, 195, 257 and references therein.

\subsection{Computation for the Spinless Relativistic Particle}

In this subsection, we show that the spinless relativistic particle of Sect. 7.1 possesses no anomaly. This example is useful for illustrating the formalism of Sect. 8.7 because the computation is relatively simple.

We use

$$
\Psi=\int \mathrm{d} \tau \overline{\mathcal{C}}(e-\rho)
$$

for the gauge-fixing fermion, where $\rho$ is an arbitrary function of $\tau$. This allows us to judge potential dependence on the gauge-fixing procedure by varying $\rho$. Next, a canonical transformation is performed to the gauge-fixed basis of Sect. 6.6 using $\Psi$ in Eq.(9.1). One obtains

$$
S \rightarrow \int \mathrm{d} \tau\left\{\frac{1}{2}\left(\frac{\dot{x}^{2}}{e}-m^{2} e\right)+x_{\mu}^{*} \dot{x}^{\mu} \frac{\mathcal{C}}{e}+\left(e^{*}+\overline{\mathcal{C}}\right) \dot{\mathcal{C}}+\bar{\pi}\left(\overline{\mathcal{C}}^{*}+e-\rho\right)\right\}
$$

It is advantageous to perform a canonical transformation that shifts $\bar{\pi}$ by the solution of the equation of motion generated by $e$. According to the result in Sect. 8.6, canonical transformations do not affect the existence or non-existence of violations 
of the quantum master equation. The variation of $S_{\Psi}$ with respect to $e$ yields

$$
\bar{\pi}-\frac{1}{2} \frac{\dot{x}^{2}}{e^{2}}-\frac{1}{2} m^{2}=0 .
$$

The relevant canonical transformation is

$$
\begin{gathered}
\bar{\pi} \rightarrow \bar{\pi}+\frac{1}{2} \frac{\dot{x}^{2}}{e^{2}}+\frac{1}{2} m^{2}, \\
e^{*} \rightarrow e^{*}+\bar{\pi}^{*} \frac{\dot{x}^{2}}{e^{3}}, \quad x_{\mu}^{*} \rightarrow x_{\mu}^{*}+\frac{d}{d \tau}\left(\frac{\bar{\pi}^{*} \dot{x}_{\mu}}{e^{2}}\right),
\end{gathered}
$$

with the other fields and antifields left unchanged. The action becomes

$$
\begin{gathered}
S \rightarrow \int \mathrm{d} \tau\left\{\left(\frac{1}{e}-\frac{\rho}{2 e^{2}}\right) \dot{x}^{2}-\frac{1}{2} \rho m^{2}+\overline{\mathcal{C}} \dot{\mathcal{C}}+\bar{\pi}(e-\rho)+\right. \\
\left.x_{\mu}^{*} \dot{x}^{\mu} \frac{\mathcal{C}}{e}+e^{*} \dot{\mathcal{C}}+\overline{\mathcal{C}}^{*} \frac{1}{2}\left(\frac{\dot{x}^{2}}{e^{2}}+m^{2}\right)-\frac{\bar{\pi}^{*} \mathcal{C} \dot{x}_{\mu}}{e^{2}} \frac{d}{d \tau}\left(\frac{\dot{x}^{\mu}}{e}\right)\right\} .
\end{gathered}
$$

Let us first determine the overall structure of the computation. From Eq.(9.5), one finds that the non-zero entries of the matrix $K_{B}^{A}$ are

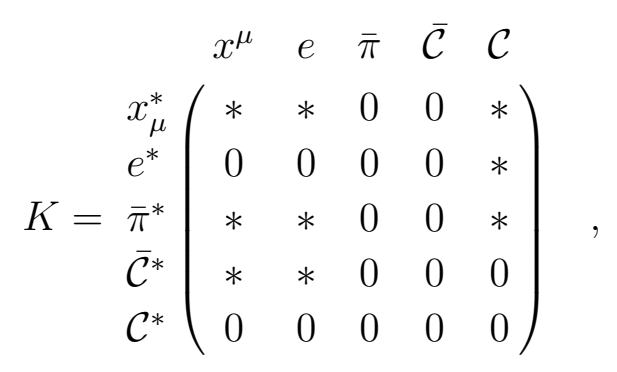

where the columns and rows are labelled by the corresponding fields and antifields. We select $T_{A B}$ to be proportional to the identity matrix, except in the ghost sector for which

$$
T_{\overline{\mathcal{C}} \mathcal{C}}=\begin{gathered}
\overline{\mathcal{C}} \\
\mathcal{C}
\end{gathered}\left(\begin{array}{cc}
0 & -1 \\
1 & 0
\end{array}\right),
$$

and in the $x$ sector for which

$$
\left(T_{x}\right)_{\mu \nu}=\eta_{\mu \nu} .
$$

In perturbation theory, the regulator $\mathcal{R}$ is evaluated at the stationary point of the gauge-fixed action. For $e$, this corresponds to

$$
\left.e\right|_{\Sigma}=\rho
$$


where $\Sigma$ indicates the stationary-point surface in field space. Using Eq.(9.5), a straightforward calculation reveals that the non-zero entries of $\left.\mathcal{R}\right|_{\Sigma}$ are

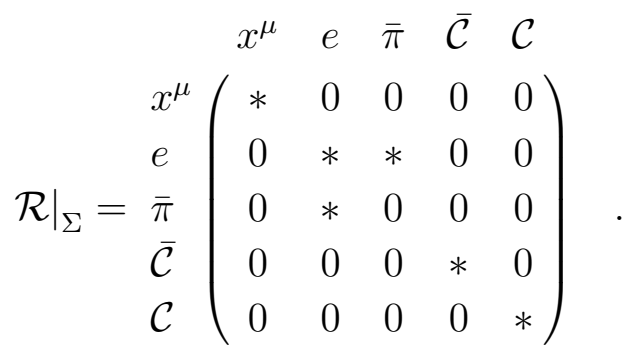

Eq.(9.10) shows that propagation is diagonal within three sectors: the $x^{\mu}$ sector, the $e-\bar{\pi}$ sector and the ghost sector. As expected, the canonical transformation in Eq.(9.4) decouples $e$ from $x^{\mu}$ : For the shifted action in Eq.(9.5), one has

$$
\left.\frac{\partial_{l} \partial_{r} S}{\partial e \partial x^{\mu}}\right|_{\Sigma}=\left.\left(-2 \frac{d}{d \tau} \frac{1}{e^{2}} \dot{x}^{\mu}+2 \frac{d}{d \tau} \frac{\rho}{e^{3}} \dot{x}^{\mu}\right)\right|_{\Sigma}=0
$$

Because a constant $T_{A B}$ matrix has been selected, the non-zero entries of $F^{A}{ }_{B}$ in Eq.(8.51) are the same as in Eq.(9.6). From the structure of Eqs.(9.6) and (9.10), one sees that the anomaly computation separates into contributions from the $x^{\mu}$ sector, the $e-\bar{\pi}$ sector and the ghost sector. The propagating fields are $x^{\mu}, \overline{\mathcal{C}}$ and $\mathcal{C}$. The field $\bar{\pi}$ serves as a Lagrange multiplier for setting $e$ equal to $\rho$. Hence, $e$ and $\bar{\pi}$ are non-propagating and should not contribute to the anomaly according to the analysis in Sect. 8.7 [253]. For this particular system, the contribution is zero because $F^{A}{ }_{B}$ in the $e-\bar{\pi}$ sector is off-diagonal. It is also clear that $\mathcal{C}$ and $\overline{\mathcal{C}}$ do not contribute to the anomaly since $F_{B}^{A}$ is zero for all ghost entries. One only needs to consider the $x^{\mu}$ sector.

In what follows we use a subscript $x$ for quantities associated with $x^{\mu}$. Applying Eqs.(8.47) and (8.48) to Eq.(9.5), one arrives at

$$
\left(K_{x}\right)_{\nu}^{\mu}=\delta^{\mu}{ }_{\nu} e^{-1} \mathcal{C} \frac{d}{d \tau}
$$

and

$$
\left(Q_{x}\right)_{\mu \nu}=\eta_{\mu \nu} Q_{x}
$$

where $Q_{x}$ without $\mu \nu$ subscripts is defined by

$$
Q_{x} \equiv-2 \frac{d}{d \tau} e^{-1} \frac{d}{d \tau}+\frac{d}{d \tau} \rho e^{-2} \frac{d}{d \tau}
$$

Here and below, the derivative $\frac{d}{d \tau}$ acts on everything to the right including $e, \rho$ and the function to which $Q_{x}$ is applied. Some contributions to Eq.(9.14) come from the 
shifts in Eq.(9.4). We have also dropped terms proportional to $\bar{\pi}^{*}$ and $\overline{\mathcal{C}}^{*}$ because they will not contribute, when expanding about the stationary point.

Since the $T_{x}$ matrix is $\left(T_{x}\right)_{\mu \nu}=\eta_{\mu \nu}$, the regulator matrix in Eq.8.52) is

$$
\left(\mathcal{R}_{x}\right)^{\mu}{ }_{\nu}=\delta^{\mu}{ }_{\nu} Q_{x}
$$

Because $\delta_{B} T_{x}=0$,

$$
\left(F_{x}\right)^{\mu}{ }_{\nu}=\left(K_{x}\right)^{\mu}{ }_{\nu} \quad,
$$

where $\left(K_{x}\right)^{\mu}{ }_{\nu}$ is given in Eq.(9.12).

All the relevant matrices of Sect. 8.7 for the computation of the anomaly have been obtained. At this stage, one expands about the stationary point of the gaugefixed action. The field $e$ is set equal to the function $\rho$ according to Eq.(9.9). The regulator matrix becomes

$$
\left.\left(\mathcal{R}_{x}\right)^{\mu}{ }_{\nu}\right|_{\Sigma}=-\delta^{\mu}{ }_{\nu} \frac{d}{d \tau} \rho^{-1} \frac{d}{d \tau},
$$

so that on-shell

$$
\begin{gathered}
(\Delta S)_{\mathrm{reg}}= \\
D \int \mathrm{d} \tau\left[\int_{-\infty}^{\infty} \frac{\mathrm{d} k}{2 \pi} \exp (-i k \tau) \rho^{-1} \mathcal{C} \frac{d}{d \tau} \exp \left(\frac{-\frac{d}{d \tau} \rho^{-1} \frac{d}{d \tau}}{\mathcal{M}^{2}}\right) \exp (i k \tau)\right]_{0},
\end{gathered}
$$

where we have used the form of $(\Delta S)_{\text {reg }}$ in Eq.(8.56). The trace over field indices $A$ produces a factor of $\int \mathrm{d} \tau$ and a factor of $D$ (because the number of $x^{\mu}$ fields is $D$ and each contributes equally). The operator trace is evaluated using a complete set of momentum-space functions, thereby generating the factors $\exp ( \pm i k \tau)$. The calculation in Eq.(9.18) is performed in Appendix C, where it is shown that the integrand is an odd function of $k$. Consequently,

$$
(\Delta S)_{\mathrm{reg}}=0 .
$$

The calculation of the $x^{\mu}$ contribution is even simpler using Eq.(8.57). To compute $\delta_{B} \mathcal{R}$, note that

$$
\delta_{B} e=(e, S)=\dot{\mathcal{C}} .
$$

Using this equation, Eq.(9.14) and Eq.(9.15), one finds

$$
\left(\delta_{B} \mathcal{R}_{x}\right)^{\mu}{ }_{\nu}=\delta^{\mu}{ }_{\nu}\left(2 \frac{d}{d \tau} e^{-2} \dot{\mathcal{C}} \frac{d}{d \tau}-2 \frac{d}{d \tau} \rho e^{-3} \dot{\mathcal{C}} \frac{d}{d \tau}\right),
$$

so that

$$
\left.\left(\delta_{B} \mathcal{R}_{x}\right)^{\mu}{ }_{\nu}\right|_{\Sigma}=0
$$


When $\delta_{B} \mathcal{R}_{x}$ is substituted into Eq.(8.57), one gets

$$
(\Delta S)_{\text {reg }}=0 \text {, }
$$

in agreement with Eq.(9.19). The absence of a violation of the quantum master equation means that the spinless relativistic particle theory is gauge-invariant even at the quantum level.

\subsection{The Abelian Chiral Schwinger Model}

In this subsection, we analyze the abelian chiral Schwinger model in two-dimensions. It is an anomalous gauge theory and a particularly simple example that illustrates the formalism of Sect. 8.7. The model contains an abelian gauge field, i. e., "a photon" $A_{\mu}$, and a charged left-handed fermion. It is governed by the following classical action

$$
S_{0}\left[A_{\mu}, \psi, \bar{\psi}\right]=\int \mathrm{d}^{2} x\left[-\frac{1}{4 e^{2}} F^{\mu \nu} F_{\mu \nu}+\bar{\psi} i \not D \psi\right],
$$

where $e$ is the electromagnetic coupling constant. Although we take $\psi$ to be a Dirac fermion, we use a covariant derivative that couples the photon only to the the rightmoving component:

$$
i \not D=i \not \supset+\not P_{-} .
$$

In other words, $P_{-} \psi$ is charged but $P_{+} \psi$ is neutral. Here $P_{ \pm}$are the chiral projectors

$$
P_{-}=\frac{1}{2}\left(1-\gamma_{5}\right), \quad P_{+}=\frac{1}{2}\left(1+\gamma_{5}\right) \quad .
$$

In two-dimensions, they project onto right- and left-moving states. Hence, the leftmoving fermion $P_{+} \psi$ is a free particle and decouples.

A slash through a vector $V_{\mu}$ represents $\gamma^{\mu} V_{\mu}: Y=\gamma^{\mu} V_{\mu}$. In two-dimensions the $\gamma^{\mu}$ are $2 \times 2$ matrices satisfying $\gamma^{\mu} \gamma^{\nu}+\gamma^{\nu} \gamma^{\mu}=-2 \eta^{\mu \nu}$, and $\gamma_{5}$ is defined as $\gamma_{5} \equiv \gamma^{0} \gamma^{1}$.

The action in Eq.(9.23) is invariant under the finite gauge transformations

$$
A_{\mu}^{\prime}=A_{\mu}+\partial_{\mu} \varepsilon, \quad \psi^{\prime}=\exp \left\{i P_{-} \varepsilon\right\} \psi, \quad \bar{\psi}^{\prime}=\bar{\psi} \exp \left\{-i P_{+} \varepsilon\right\} .
$$

Although this model has not been discussed in previous sections, it is straightforward to apply the field-antifield formalism [61, 63]. The proper solution for the gauge sector corresponds to the solution for the Yang-Mills example given in Sect. 5.2 for $d=2$ and for a $U(1)$ group. In addition to the antifield $A_{\mu}^{*}$ of the photon, one has commuting antifields $\psi^{*}$ and $\bar{\psi}^{*}$ for the fermions. The proper solution of the master equation is

$$
S=S_{0}+\int \mathrm{d}^{2} x\left[A_{\mu}^{*} \partial^{\mu} \mathcal{C}+i\left(\psi^{*}\right)^{t} P_{-} \psi \mathcal{C}-i \bar{\psi}^{*} P_{+}^{t} \bar{\psi}^{t} \mathcal{C}\right]
$$


where $\mathcal{C}$ is the ghost field associated with the gauge parameter $\varepsilon$. The superscript $t$ stands for transpose: $\psi=\left(\begin{array}{c}\psi^{1} \\ \psi^{2}\end{array}\right)$ and $\bar{\psi}=\left(\bar{\psi}^{1}, \bar{\psi}^{2}\right)$, so that $\bar{\psi}^{t}=\left(\begin{array}{c}\bar{\psi}^{1} \\ \bar{\psi}^{2}\end{array}\right)$ and $\left(\psi^{*}\right)^{t}=\left(\left(\psi^{1}\right)^{*},\left(\psi^{2}\right)^{*}\right)$.

A formal computation using the expression for $\Delta$ in Eq.(6.7) reveals that only the fermion sector contributes to $\Delta S$. A more detailed analysis using a regularization procedure confirms this. Therefore, we focus on the contribution to $\Delta S$ from $\psi$ and $\bar{\psi}$. Gauge-fixing is not needed because propagators for the fermions already exist. For these reasons, it is not necessary to consider gauge-fixing auxiliary fields, nor a non-minimal proper solution.

Using Eqs.(8.47) and (9.27), one finds that the $K$ matrix is given by

$$
K=-i \mathcal{C}\left(\begin{array}{cc}
P_{-} & 0_{2} \\
0_{2} & -P_{+}^{t}
\end{array}\right)
$$

where all entries are $2 \times 2$ matrices, e. g., $0_{2}=\left(\begin{array}{ll}0 & 0 \\ 0 & 0\end{array}\right)$, so that $K$ is a $4 \times 4$ matrix. In Eq.(9.28) and throughout this subsection, we label the rows and columns of matrices in the order $\psi^{1}, \psi^{2}, \bar{\psi}^{1}, \bar{\psi}^{2}$. From Eqs.(8.48) and (9.23), the matrix $Q$ for the fermion sector is

$$
Q=\left(\begin{array}{cc}
0_{2} & -i \tilde{D} \\
i \not D & 0_{2}
\end{array}\right)
$$

where $\tilde{\not D}$ is defined by $\int \mathrm{d}^{2} x \bar{\psi} i \not D \psi=-\int \mathrm{d}^{2} x \psi^{t} i \tilde{D} \bar{\psi}^{t}$. More precisely,

$$
i \tilde{D}=i \not \partial^{t}-A^{t} P_{+}^{t}
$$

where, here, the superscript $t$ indicates the transpose of a matrix in Dirac-index space.

For the matrix $T$, we choose

$$
T=\left(\begin{array}{cc}
0_{2} & 1_{2} \\
-1_{2} & 0_{2}
\end{array}\right)
$$

where $1_{2}=\left(\begin{array}{ll}1 & 0 \\ 0 & 1\end{array}\right)$. Because $\delta_{B} T=0$, the matrix $F$ in Eq.(8.51) is equal to the matrix $K$ in Eq.(9.28). Using Eq.(8.49), one finds

$$
\mathcal{O}=\left(\begin{array}{cc}
i \not D & 0_{2} \\
0_{2} & i \tilde{D}
\end{array}\right)
$$

${ }^{12}$ The computation made in ref.[78] for a pure Yang-Mills theories in four dimensions supports the idea that gauge fields and ghosts produce a BRST trivial contribution to $(\Delta S)_{\text {reg }}$. 
Then from Eq.(8.54), one obtains

$$
R=\left(\begin{array}{cc}
-\not D D D & 0_{2} \\
0_{2} & -\tilde{D} \tilde{D}
\end{array}\right),
$$

for the regulator matrix.

Let us use the following representation of the gamma matrices:

$$
\gamma^{0}=\sigma^{1}=\left(\begin{array}{ll}
0 & 1 \\
1 & 0
\end{array}\right), \quad \gamma^{1}=i \sigma^{2}=\left(\begin{array}{cc}
0 & 1 \\
-1 & 0
\end{array}\right),
$$

so that

$$
\gamma^{5}=\gamma^{0} \gamma^{1}=-\sigma^{3}=\left(\begin{array}{cc}
-1 & 0 \\
0 & 1
\end{array}\right)
$$

and

$$
P_{-}=\left(\begin{array}{ll}
1 & 0 \\
0 & 0
\end{array}\right), \quad P_{+}=\left(\begin{array}{ll}
0 & 0 \\
0 & 1
\end{array}\right) .
$$

With this representation,

$$
i \not D=\left(\begin{array}{cc}
0 & i \partial_{+} \\
i \partial_{-}+A_{-} & 0
\end{array}\right), \quad i \tilde{D}=\left(\begin{array}{cc}
0 & i \partial_{-}-A_{-} \\
i \partial_{+} & 0
\end{array}\right),
$$

where

$$
\partial_{ \pm}=\partial_{0} \pm \partial_{1}, \quad A_{-}=A_{0}-A_{1}
$$

The entries in $R$ of Eq.9.33) are

$$
\begin{aligned}
& -\not D D D \\
& =\left(\begin{array}{cc}
i \partial_{+}\left(i \partial_{-}+A_{-}\right) & 0 \\
0 & \left(i \partial_{-}+A_{-}\right) i \partial_{+}
\end{array}\right), \\
& -\tilde{D} \tilde{D}=\left(\begin{array}{cc}
\left(i \partial_{-}-A_{-}\right) i \partial_{+} & 0 \\
0 & i \partial_{+}\left(i \partial_{-}-A_{-}\right)
\end{array}\right) .
\end{aligned}
$$

The derivative $\partial_{+}$acts to the right, so that it differentiates $A_{-}$as well as any function to which $R$ is applied. Note that $R$ is diagonal.

In general, the matrix $F^{\prime}$ in Eq. (8.55) has two contributions. However, the term proportial to $\delta_{B} \mathcal{O}$ does not contribute, upon taking the trace, because $\mathcal{O}$ is offdiagonal and $R$ is diagonal when the above gamma matrices are used. Hence, one may take

$$
F^{\prime}=F=K,
$$

where $K$ is given in Eq.(9.28). Summarizing, for the computation of $\Delta S$ in Eq.(8.56), one uses $F=K$ in Eq.(9.28) and $R$ given by Eqs. (9.33) and (9.39). Incorporating the projectors $P_{ \pm}$in $K$, one obtains

$$
\Delta S=-i\left[\operatorname{Tr} \mathcal{C}\left(\exp \left(\frac{R_{+}}{\mathcal{M}^{2}}\right)-\exp \left(\frac{R_{-}}{\mathcal{M}^{2}}\right)\right)\right]_{0},
$$


where there is no trace in Dirac-index space, only in function space. In Eq.(9.41),

$$
R_{ \pm}=\partial^{\mu} \partial_{\mu} \pm i\left(\partial_{+} A_{-}\right) \pm i A_{-} \partial_{+} \quad .
$$

where we use a parenthesis around $\left(\partial_{+} A_{-}\right)$to indicate that $\partial_{+}$acts only on $A_{-}$.

In Appendix C, the computation of Eq.(9.42) is performed. One finds

$$
\mathcal{A}_{1}=\Delta S=\frac{i}{4 \pi} \int \mathrm{d}^{2} x \mathcal{C}\left(\epsilon^{\mu \nu} \partial_{\mu} A_{\nu}-\partial_{\mu} A^{\mu}\right)
$$

where $\epsilon^{10}=1=-\epsilon^{01}$. Note that the anomaly is consistent since

$$
\delta_{B} \mathcal{A}_{1}=\frac{i}{4 \pi} \int \mathrm{d}^{2} x \mathcal{C}\left(\epsilon^{\mu \nu} \partial_{\mu} \partial_{\nu} \mathcal{C}-\partial_{\mu} \partial^{\mu} \mathcal{C}\right)=0 .
$$

The first term vanishes by the antisymmetry property of $\epsilon^{\mu \nu}$, while the second term vanishes by integration by parts and the anticommuting nature of $\mathcal{C}$. A local counterterm $\Omega_{1}$ cannot be added to the action to eliminate $\mathcal{A}_{1}$ via Eq.(8.40). If one takes $\Omega_{1}=-\frac{1}{8 \pi} \int \mathrm{d}^{2} x A^{\mu} A_{\mu}$, then the second term in Eq.(9.43) is eliminated, but the term $\frac{i}{8 \pi} \int \mathrm{d}^{2} x \mathcal{C} \epsilon^{\mu \nu} F_{\mu \nu}$ in $\mathcal{A}_{1}$ remains.

\subsection{Anomaly in the Open Bosonic String}

In this subsection, we investigate the violation of the quantum master equation for the first-quantized open bosonic string when the dimension of space-time is not 26. The bosonic contribution was explicitly computed in refs. [251, 133]. We gauge-fix the action using the fermion $\Psi$ in Eq.(7.42). It depends on the conformal factor $\rho$. It turns out that when $D \neq 26$, there is an anomaly. As a consequence, the theory depends on $\rho$. Although the theory is classically gauge-invariant, one of the four gauge symmetries is violated by quantum effects. This anomalous gauge symmetry cannot be fixed for $D \neq 26$.

Let us apply the one-loop anomaly analysis given in Sect. 8.7 to the bosonic string. First, we perform a canonical transformation with $\Psi$ so that $\Phi_{A}^{*} \rightarrow \Phi_{A}^{*}+\frac{\partial \Psi}{\partial \Phi^{A}}$. This leads to the following shifts in antifield fields

$$
\begin{array}{rlrl}
e^{* \tau} & \rightarrow e^{* \tau}+\overline{\mathcal{C}}+\overline{\mathcal{C}}_{\sigma}, & e^{* \sigma}{ }_{\sigma} \rightarrow e^{* \sigma}{ }_{\sigma}+\overline{\mathcal{C}}-\overline{\mathcal{C}}_{\sigma}, \\
e^{* \tau}{ }_{\sigma} \rightarrow e^{* \tau}+\overline{\mathcal{C}}_{\tau \sigma}+\overline{\mathcal{C}}_{\tau}, & e^{* \sigma} \rightarrow e^{* \sigma}+\overline{\mathcal{C}}_{\tau \sigma}-\overline{\mathcal{C}}_{\tau}, \\
\overline{\mathcal{C}}^{* \tau} \rightarrow \overline{\mathcal{C}}^{* \tau}+e_{\tau}{ }^{\sigma}-e_{\sigma}{ }^{\tau}, & \overline{\mathcal{C}}^{* \sigma} & \rightarrow \overline{\mathcal{C}}^{* \sigma}+e_{\tau}{ }^{\tau}-e_{\sigma}{ }^{\sigma}, \\
\overline{\mathcal{C}}^{*} \rightarrow \overline{\mathcal{C}}^{*}+e_{\tau}^{\tau}+e_{\sigma}{ }^{\sigma}-2 \rho^{-1 / 2}, & \overline{\mathcal{C}}^{* \tau \sigma} & \rightarrow \overline{\mathcal{C}}^{* \tau \sigma}+e_{\tau}{ }^{\sigma}+e_{\sigma}{ }^{\tau} .
\end{array}
$$


Additional terms are produced in the total action $S_{\text {total }}=S+S_{\text {aux }}$ of Eqs.(5.30) and (7.41) given by

$$
\begin{aligned}
& S \rightarrow S+\int \mathrm{d} \tau \int_{0}^{\pi} \mathrm{d} \sigma\left\{\left(\overline{\mathcal{C}}+\overline{\mathcal{C}}_{\sigma}\right)\left(\mathcal{C}^{n} \partial_{n} e_{\tau}^{\tau}-e_{\tau}^{n} \partial_{n} \mathcal{C}^{\tau}\right)+\left(\overline{\mathcal{C}}-\overline{\mathcal{C}}_{\sigma}\right)\left(\mathcal{C}^{n} \partial_{n} e_{\sigma}{ }^{\sigma}-e_{\sigma}{ }^{n} \partial_{n} \mathcal{C}^{\sigma}\right)\right. \\
&+\left(\overline{\mathcal{C}}_{\tau \sigma}+\overline{\mathcal{C}}_{\tau}\right)\left(\mathcal{C}^{n} \partial_{n} e_{\tau}{ }^{\sigma}-e_{\tau}^{n} \partial_{n} \mathcal{C}^{\sigma}\right)+\left(\overline{\mathcal{C}}_{\tau \sigma}-\overline{\mathcal{C}}_{\tau}\right)\left(\mathcal{C}^{n} \partial_{n} e_{\sigma}{ }^{\tau}-e_{\sigma}{ }^{n} \partial_{n} \mathcal{C}^{\tau}\right) \\
&+\left(\left(\overline{\mathcal{C}}+\overline{\mathcal{C}}_{\sigma}\right) e_{\tau}^{\tau}+\left(\overline{\mathcal{C}}-\overline{\mathcal{C}}_{\sigma}\right) e_{\sigma}{ }^{\sigma}+\left(\overline{\mathcal{C}}_{\tau \sigma}+\overline{\mathcal{C}}_{\tau}\right) e_{\tau}{ }^{\sigma}+\left(\overline{\mathcal{C}}_{\tau \sigma}-\overline{\mathcal{C}}_{\tau}\right) e_{\sigma}{ }^{\tau}\right) \mathcal{C} \\
&\left.-\left(\left(\overline{\mathcal{C}}+\overline{\mathcal{C}}_{\sigma}\right) e_{\sigma}^{\tau}+\left(\overline{\mathcal{C}}-\overline{\mathcal{C}}_{\sigma}\right) e_{\tau}{ }^{\sigma}+\left(\overline{\mathcal{C}}_{\tau \sigma}+\overline{\mathcal{C}}_{\tau}\right) e_{\sigma}{ }^{\sigma}+\left(\overline{\mathcal{C}}_{\tau \sigma}-\overline{\mathcal{C}}_{\tau}\right) e_{\tau}^{\tau}\right) \mathcal{C}^{\tau \sigma}\right\}
\end{aligned}
$$

and

$$
\begin{gathered}
S_{\mathrm{aux}} \rightarrow \int \mathrm{d} \tau \int_{0}^{\pi} \mathrm{d} \sigma\left\{\bar{\pi}\left(\overline{\mathcal{C}}^{*}+e_{\tau}^{\tau}+e_{\sigma}{ }^{\sigma}-2 \rho^{-1 / 2}\right)+\right. \\
\left.\bar{\pi}_{\tau}\left(\overline{\mathcal{C}}^{* \tau}+e_{\tau}^{\sigma}-e_{\sigma}^{\tau}\right)+\bar{\pi}_{\sigma}\left(\overline{\mathcal{C}}^{* \sigma}+e_{\tau}^{\tau}-e_{\sigma}^{\sigma}\right)+\bar{\pi}_{\tau \sigma}\left(\overline{\mathcal{C}}^{* \tau \sigma}+e_{\tau}{ }^{\sigma}+e_{\sigma}{ }^{\tau}\right)\right\} .
\end{gathered}
$$

At this stage, it is desirable to shift fields by the solutions to the equations of motion of the $e_{a}{ }^{m}$, by using a canonical transformation. Such a shift guarantees that the quadratic form $Q_{A B}$ is on-shell diagonal in the $e_{a}{ }^{m}$ sector. This avoids mixing of the $X^{\mu}$ and ghost sectors with the $\bar{\pi}-e_{a}{ }^{m}$ sector. Variations of $S_{\text {total }}$ in the gauge-fixed basis with respect to the $e_{a}{ }^{m}$ produce the equations for the four $\bar{\pi}$. Using a subscript 0 to denote the solutions, and ignoring terms proportional to antifields, one finds that

$$
\begin{gathered}
2(\bar{\pi})_{0} \equiv-\left(\frac{\partial S}{\partial e_{\tau}{ }^{\tau}}+\frac{\partial S}{\partial e_{\sigma}{ }^{\sigma}}-2 \bar{\pi}\right)= \\
\left(e_{\tau}^{\tau}+e_{\sigma}^{\sigma}\right) e \mathcal{L}_{X}-e\left(\partial_{\tau} X^{\mu} D_{\tau} X_{\mu}-\partial_{\sigma} X^{\mu} D_{\sigma} X_{\mu}\right)+ \\
2 \partial_{n}\left(\overline{\mathcal{C}} \mathcal{C}^{n}\right)+\overline{\mathcal{C}}\left(\partial_{n} \mathcal{C}^{n}-2 \mathcal{C}\right)+\overline{\mathcal{C}}_{\tau \sigma}\left(\partial_{\tau} \mathcal{C}^{\sigma}+\partial_{\sigma} \mathcal{C}^{\tau}+2 \mathcal{C}^{\tau \sigma}\right)+ \\
\overline{\mathcal{C}}_{\tau}\left(\partial_{\tau} \mathcal{C}^{\sigma}-\partial_{\sigma} \mathcal{C}^{\tau}\right)+\overline{\mathcal{C}}_{\sigma}\left(\partial_{\tau} \mathcal{C}^{\tau}-\partial_{\sigma} \mathcal{C}^{\sigma}\right), \\
2\left(\bar{\pi}_{\tau \sigma}\right)_{0} \equiv-\left(\frac{\partial S}{\partial e_{\tau}{ }^{\sigma}}+\frac{\partial S}{\partial e_{\sigma}{ }^{\tau}}-2 \bar{\pi}_{\tau \sigma}\right)= \\
-\left(e_{\tau}{ }^{\sigma}+e_{\sigma}{ }^{\tau}\right) e \mathcal{L}_{X}-e\left(\partial_{\sigma} X^{\mu} D_{\tau} X_{\mu}-\partial_{\tau} X^{\mu} D_{\sigma} X_{\mu}\right)+ \\
2 \partial_{n}\left(\overline{\mathcal{C}}_{\tau \sigma} \mathcal{C}^{n}\right)+\overline{\mathcal{C}}\left(\partial_{\tau} \mathcal{C}^{\sigma}+\partial_{\sigma} \mathcal{C}^{\tau}+2 \mathcal{C}^{\tau \sigma}\right)+\overline{\mathcal{C}}_{\tau \sigma}\left(\partial_{n} \mathcal{C}^{n}-2 \mathcal{C}\right) \\
-\overline{\mathcal{C}}_{\tau}\left(\partial_{\tau} \mathcal{C}^{\tau}-\partial_{\sigma} \mathcal{C}^{\sigma}\right)-\overline{\mathcal{C}}_{\sigma}\left(\partial_{\tau} \mathcal{C}^{\sigma}-\partial_{\sigma} \mathcal{C}^{\tau}\right) \\
2\left(\bar{\pi}_{\tau}\right)_{0} \equiv-\left(\frac{\partial S}{\partial e_{\tau}{ }^{\sigma}}-\frac{\partial S}{\partial e_{\sigma} \tau}-2 \bar{\pi}_{\tau}\right)= \\
\left(e_{\tau}{ }^{\sigma}-e_{\sigma}{ }^{\tau}\right) e \mathcal{L}_{X}-e\left(\partial_{\sigma} X^{\mu} D_{\tau} X_{\mu}+\partial_{\tau} X^{\mu} D_{\sigma} X_{\mu}\right)+
\end{gathered}
$$




$$
\begin{gathered}
2 \partial_{n}\left(\overline{\mathcal{C}}_{\tau} \mathcal{C}^{n}\right)-\overline{\mathcal{C}}\left(\partial_{\tau} \mathcal{C}^{\sigma}-\partial_{\sigma} \mathcal{C}^{\tau}\right)-\overline{\mathcal{C}}_{\tau \sigma}\left(\partial_{\tau} \mathcal{C}^{\tau}-\partial_{\sigma} \mathcal{C}^{\sigma}\right) \\
+\overline{\mathcal{C}}_{\tau}\left(\partial_{n} \mathcal{C}^{n}-2 \mathcal{C}\right)+\overline{\mathcal{C}}_{\sigma}\left(\partial_{\tau} \mathcal{C}^{\sigma}+\partial_{\sigma} \mathcal{C}^{\tau}-2 \mathcal{C}^{\tau \sigma}\right) \\
2\left(\bar{\pi}_{\sigma}\right)_{0} \equiv-\left(\frac{\partial S}{\partial e_{\tau} \tau}-\frac{\partial S}{\partial e_{\sigma}{ }^{\sigma}}-2 \bar{\pi}_{\sigma}\right)= \\
\left(e_{\sigma}{ }^{\sigma}-e_{\tau}^{\tau}\right) e \mathcal{L}_{X}-e\left(\partial_{\tau} X^{\mu} D_{\tau} X_{\mu}+\partial_{\sigma} X^{\mu} D_{\sigma} X_{\mu}\right)+ \\
2 \partial_{n}\left(\overline{\mathcal{C}}_{\sigma} \mathcal{C}^{n}\right)+\overline{\mathcal{C}}\left(\partial_{\tau} \mathcal{C}^{\tau}-\partial_{\sigma} \mathcal{C}^{\sigma}\right)+\overline{\mathcal{C}}_{\tau \sigma}\left(\partial_{\tau} \mathcal{C}^{\sigma}-\partial_{\sigma} \mathcal{C}^{\tau}\right) \\
+\overline{\mathcal{C}}_{\tau}\left(\partial_{\tau} \mathcal{C}^{\sigma}+\partial_{\sigma} \mathcal{C}^{\tau}-2 \mathcal{C}^{\tau \sigma}\right)+\overline{\mathcal{C}}_{\sigma}\left(\partial_{n} \mathcal{C}^{n}-2 \mathcal{C}\right)
\end{gathered}
$$

where the $X^{\mu}$-lagrangian density $\mathcal{L}_{X}$ is defined to be

$$
\mathcal{L}_{X} \equiv \frac{e}{2}\left(D_{\tau} X^{\mu} D_{\tau} X_{\mu}-D_{\sigma} X^{\mu} D_{\sigma} X_{\mu}\right)
$$

The canonical transformation of interest is given by

$$
\begin{gathered}
\bar{\pi} \rightarrow \bar{\pi}+(\bar{\pi})_{0}, \quad \bar{\pi}_{n} \rightarrow \bar{\pi}_{n}+\left(\bar{\pi}_{n}\right)_{0}, \quad \bar{\pi}_{\tau \sigma} \rightarrow \bar{\pi}_{\tau \sigma}+\left(\bar{\pi}_{\tau \sigma}\right)_{0} \\
\Phi_{A}^{*} \rightarrow \Phi_{A}^{*}-\bar{\pi}^{*} \frac{\partial_{r}(\bar{\pi})_{0}}{\partial \Phi^{A}}-\bar{\pi}^{* \tau} \frac{\partial_{r}\left(\bar{\pi}_{\tau}\right)_{0}}{\partial \Phi^{A}}-\bar{\pi}^{* \sigma} \frac{\partial_{r}\left(\bar{\pi}_{\sigma}\right)_{0}}{\partial \Phi^{A}}-\bar{\pi}^{* \tau \sigma} \frac{\partial_{r}\left(\bar{\pi}_{\tau \sigma}\right)_{0}}{\partial \Phi^{A}}
\end{gathered}
$$

where in the first equation, $n$ stands for $\tau$ or $\sigma$.

The goal of the next few paragraphs is to obtain $F^{A}{ }_{B}$ and $\mathcal{R}^{A}{ }_{B}$ so that the anomaly in Eq. (8.53) can be computed. One must first calculate $K^{A}{ }_{B}$ and $Q_{A B}$ and specify $T_{A B}$. Simplifications occur for the following reasons. In the final gauge-fixed form of the action in Eq.(7.45), the propagating fields are $X^{\mu}, \overline{\mathcal{C}}_{n}$ and $\mathcal{C}^{n}$ where $n$ represents $\tau$ and $\sigma .3$ By the argument in Sect. 8.7, only these fields contribute; the $F_{B}^{A}$ and $\mathcal{R}_{B}^{A}$ associated with non-propagating fields do not enter the calculation 253]. Furthermore, by a judicial choice of $T_{A B}, \mathcal{R}^{A}{ }_{B}$ can be made diagonal. Hence, only the diagonal components of $K^{A}{ }_{B}, \delta_{B} \mathcal{O}^{A}{ }_{B}$ and $F^{A}{ }_{B}$ for $X^{\mu}, \overline{\mathcal{C}}_{n}$ and $\mathcal{C}^{n}$ need to be computed. In what follows, we use a subscript $X, \overline{\mathcal{C}}$ and $\mathcal{C}$ to denote respectively a matrix restricted to the subspace corresponding to $X^{\mu}, \overline{\mathcal{C}}_{n}$ and $\mathcal{C}^{n}$. The subscript "ghost" is used to denote the combined $\overline{\mathcal{C}}_{n}$ and $\mathcal{C}^{n}$ subspace.

After the above two canonical transformations have been performed, the computation is straightforward. From Eq.(8.47), one finds that the $K_{B}^{A}$ in the three sectors are

$$
\left(K_{X}\right)^{\mu}{ }_{\nu}=\delta^{\mu}{ }_{\nu} \mathcal{C}^{n} \partial_{n}
$$

${ }^{13}$ This is made clear by setting $\mathcal{C}=\hat{\mathcal{C}}+\frac{1}{2}\left(\partial_{\tau} \mathcal{C}^{\tau}+\partial_{\sigma} \mathcal{C}^{\sigma}\right)-2 \rho^{1 / 2} \mathcal{C}^{n} \partial_{n} \rho^{-1 / 2}$ and $\mathcal{C}^{\tau \sigma}=\hat{\mathcal{C}}^{\tau \sigma}-$ $\frac{1}{2}\left(\partial_{\tau} \mathcal{C}^{\sigma}+\partial_{\sigma} \mathcal{C}^{\tau}\right)$. Throughout this subsection, one should think of $\mathcal{C}$ and $\mathcal{C}^{\tau \sigma}$ as standing for these combinations of fields. In terms of $\hat{\mathcal{C}}$ and $\hat{\mathcal{C}}^{\tau \sigma}$, the gauge-fixed action in Eq. (7.45) is block diagonal and $\overline{\mathcal{C}}, \overline{\mathcal{C}}^{\tau \sigma}, \hat{\mathcal{C}}$ and $\hat{\mathcal{C}}^{\tau \sigma}$ are non-propagating fields. The calculations in this subsection should be performed in terms of $\hat{\mathcal{C}}$ and $\hat{\mathcal{C}}^{\tau \sigma}$. At the end of the computation, one sets $\hat{\mathcal{C}}=\mathcal{C}-\frac{1}{2}\left(\partial_{\tau} \mathcal{C}^{\sigma}-\partial_{\sigma} \mathcal{C}^{\tau}\right)+$ $2 \rho^{1 / 2} \mathcal{C}^{n} \partial_{n} \rho^{-1 / 2}$ and $\hat{\mathcal{C}}^{\tau \sigma}=\mathcal{C}^{\tau \sigma}+\frac{1}{2}\left(\partial_{\tau} \mathcal{C}^{\sigma}-\partial_{\sigma} \mathcal{C}^{\tau}\right)$, thereby returning to the original fields. 


$$
\begin{gathered}
\left(K_{\overline{\mathcal{C}}}\right)_{\tau}^{\tau}=\left(K_{\overline{\mathcal{C}}}\right)_{\sigma}^{\sigma}=-\mathcal{C}^{n} \partial_{n}-\frac{3}{2}\left(\partial_{n} \mathcal{C}^{n}\right)+\mathcal{C}, \\
\left(K_{\mathcal{C}}\right)_{\tau}^{\tau}=-\mathcal{C}^{n} \partial_{n}+\frac{1}{2}\left(\partial_{n} \mathcal{C}^{n}\right)+\frac{1}{2}\left(\partial_{\tau} C^{\tau}-\partial_{\sigma} C^{\sigma}\right), \\
\left(K_{\mathcal{C}}\right)_{\sigma}^{\sigma}=-\mathcal{C}^{n} \partial_{n}+\frac{1}{2}\left(\partial_{n} \mathcal{C}^{n}\right)-\frac{1}{2}\left(\partial_{\tau} C^{\tau}-\partial_{\sigma} C^{\sigma}\right),
\end{gathered}
$$

where the presence of a parenthesis around a derivative indicates that it acts only on fields within the parenthesis. Throughout this subsection, the absence of a parenthesis means that the derivative acts on everything to the right. The terms for $K_{X}$ and $K_{\mathcal{C}}$ arise from differentiating Eq.(5.30), whereas those for $K_{\overline{\mathcal{C}}}$ come from $S_{\text {aux }}$ in Eq. (9.47) after the $\bar{\pi}_{n}$ shifts in Eq.9.50) have been performed.

The quadratic forms $Q_{A B}$ are

$$
\left(Q_{X}\right)_{\mu \nu}=\eta_{\mu \nu} Q_{X}
$$

where

$$
\begin{aligned}
Q_{X}= & D_{\tau}^{\dagger} e\left(1-e \rho^{-1 / 2}\left(e_{\tau}{ }^{\tau}+e_{\sigma}{ }^{\sigma}\right)\right) D_{\tau}-D_{\sigma}^{\dagger} e\left(1-e \rho^{-1 / 2}\left(e_{\tau}{ }^{\tau}+e_{\sigma}{ }^{\sigma}\right)\right) D_{\sigma} \\
& +D_{\tau}^{\dagger} e \rho^{-1 / 2} \partial_{\tau}-\partial_{\tau} e \rho^{-1 / 2} D_{\tau}-D_{\sigma}^{\dagger} e \rho^{-1 / 2} e \partial_{\sigma}+\partial_{\sigma} e \rho^{-1 / 2} D_{\sigma}
\end{aligned}
$$

for the $X^{\mu}$ sector, where the covariant derivatives $D_{\tau}$ and $D_{\sigma}$ are given in Eq.(3.71), and where $D_{\tau}^{\dagger}=-\partial_{\tau} e_{\tau}^{\tau}-\partial_{\sigma} e_{\tau}{ }^{\sigma}$ and $D_{\sigma}^{\dagger}=-\partial_{\tau} e_{\sigma}{ }^{\tau}-\partial_{\sigma} e_{\sigma}{ }^{\sigma}$. Since derivatives act to the right, $\partial_{\tau}$ and $\partial_{\sigma}$ in $D_{\tau}^{\dagger}$ in first the term in Eq.(9.53) act on $e\left(1-e \rho^{-1 / 2}\left(e_{\tau}^{\tau}+e_{\sigma}^{\sigma}\right)\right)$, on the vielbeins in $D_{\tau}^{\dagger}$ and $D_{\tau}$, and on any function to which the operator $Q_{X}$ is applied. Likewise, for the other derivatives. The terms in Eq.(9.53) come from the original action $S_{0}$ in Eq.(3.79), as well as from the $\bar{\pi}$ shifts of Eq.(9.50) in $S_{\text {aux }}$ of Eq.(9.47). For the ghost sector, let

$$
V=\left(\begin{array}{l}
\overline{\mathcal{C}}_{\tau} \\
\overline{\mathcal{C}}_{\sigma} \\
C^{\tau} \\
C^{\sigma}
\end{array}\right)
$$

Then the ghost part of the action is

$$
S_{\text {ghost }}=\frac{1}{2} \int \mathrm{d} \tau \int_{0}^{\pi} \mathrm{d} \sigma V^{t} Q_{\text {ghost }} V,
$$

where the superscript $t$ on $V^{t}$ stands for transpose. The ghost quadratic form is

$$
Q_{\text {ghost }}=\left(\begin{array}{llll}
0 & 0 & \rho^{-1 / 2} \partial_{\sigma} & -\rho^{-1 / 2} \partial_{\tau} \\
0 & 0 & -\rho^{-1 / 2} \partial_{\tau} & \rho^{-1 / 2} \partial_{\sigma} \\
\partial_{\sigma} \rho^{-1 / 2} & -\partial_{\tau} \rho^{-1 / 2} & 0 & 0 \\
-\partial_{\tau} \rho^{-1 / 2} & \partial_{\sigma} \rho^{-1 / 2} & 0 & 0
\end{array}\right) .
$$


The terms in $Q_{\text {ghost }}$ originate from Eq.(9.46) and from the $\bar{\pi}$ shifts of Eq.(9.50) in $S_{\text {aux }}$ of Eq.(9.47). The dependence on $e_{a}{ }^{m}$ cancels between the two contributions leaving only a dependence on $\rho$.

For the matrix $T_{A B}$ of Sect. 8.7, we choose

$$
\left(T_{X}\right)_{\mu \nu}=e \eta_{\mu \nu}
$$

and

$$
T_{\text {ghost }}=-i\left(\begin{array}{llll}
0 & e^{-2} & 0 & 0 \\
-e^{-2} & 0 & 0 & 0 \\
0 & 0 & 0 & e^{2} \\
0 & 0 & -e^{2} & 0
\end{array}\right),
$$

where $T_{\text {ghost }}$ acts in the space of antighosts and ghosts given in Eq.(9.54). In the $X^{\mu}$ sector the operator $\mathcal{O}$, defined in Eq. 8.49), is $e^{-1} \delta^{\mu}{ }_{\nu} Q_{X}$ and is equal to $\left(\mathcal{R}_{X}\right)^{\mu}{ }_{\nu}$, where $Q_{X}$ is given in Eq.(9.53). In the ghost sector, $\mathcal{O}$ is

$$
\mathcal{O}_{\text {ghost }}=i\left(\begin{array}{ll}
0_{2} & e^{2} \rho^{-1 / 2} \not \partial \\
e^{-2} \not \partial \rho^{-1 / 2} & 0_{2}
\end{array}\right),
$$

where each entry is a two by two matrix. It is somewhat accidental that the Dirac operator in two-dimensional Minkowski space

$$
\not \partial \equiv \gamma^{n} \partial_{n}=\gamma^{\tau} \partial_{\tau}+\gamma^{\sigma} \partial_{\sigma} \quad,
$$

enters in Eq.(9.59), if the gamma matrices

$$
\gamma^{\tau}=\sigma^{3}=\left(\begin{array}{ll}
1 & 0 \\
0 & -1
\end{array}\right), \quad \gamma^{\sigma}=-i \sigma^{2}=\left(\begin{array}{ll}
0 & -1 \\
1 & 0
\end{array}\right)
$$

are used. They satisfy

$$
\gamma^{n} \gamma^{m}+\gamma^{m} \gamma^{n}=-2 \eta^{n m} .
$$

The ghost-sector regulator matrix $\mathcal{R}_{\text {ghost }}$ is

$$
\mathcal{R}_{\text {ghost }}=\mathcal{O}^{2}=\left(\begin{array}{ll}
\mathcal{R}_{\overline{\mathcal{C}}} & 0_{2} \\
0_{2} & \mathcal{R}_{\mathcal{C}}
\end{array}\right)
$$

where

$$
\mathcal{R}_{\overline{\mathcal{C}}}=-e^{2} \rho^{-1 / 2} \not \partial e^{-2} \not \partial \rho^{-1 / 2}, \quad \mathcal{R}_{\mathcal{C}}=-e^{-2} \not \partial e^{2} \rho^{-1} \not \partial
$$

To compute $F^{A}{ }_{C}$ in Eq.(8.51), one needs the BRST variation of $T_{A B}$. Using

$$
\delta_{B} e=\mathcal{C}^{n} \partial_{n} e+e \partial_{n} \mathcal{C}^{n}-2 e \mathcal{C}
$$


and Eqs.(9.57) and (9.58), it is straightforward to calculate $\left(T^{-1}\right)^{A D}\left(\delta_{B} T\right)_{D C}$. The result is combined with $K^{A}{ }_{C}$ in Eq.9.51) to arrive at

$$
\left(F_{X}\right)^{\mu}{ }_{\nu}=\delta^{\mu}{ }_{\nu} F_{X}
$$

where

$$
F_{X}=\mathcal{C}^{n} \partial_{n}+\frac{1}{2}\left(\partial_{n} \mathcal{C}^{n}\right)+\frac{1}{2} e^{-1} \mathcal{C}^{n}\left(\partial_{n} e\right)-\mathcal{C},
$$

for the $X^{\mu}$ sector. For the ghost sector

$$
\begin{gathered}
\left(F_{\overline{\mathcal{C}}}\right)^{\tau}{ }_{\tau}=\left(F_{\overline{\mathcal{C}}}\right)^{\sigma}{ }_{\sigma}=-\mathcal{C}^{n} \partial_{n}-\frac{1}{2}\left(\partial_{n} \mathcal{C}^{n}\right)+e^{-1} \mathcal{C}^{n}\left(\partial_{n} e\right)-\mathcal{C} \\
\left(F_{\mathcal{C}}\right)_{\tau}^{\tau}=-\mathcal{C}^{n} \partial_{n}-\frac{1}{2}\left(\partial_{n} \mathcal{C}^{n}\right)-e^{-1} \mathcal{C}^{n}\left(\partial_{n} e\right)+2 \mathcal{C}+\frac{1}{2}\left(\partial_{\tau} \mathcal{C}^{\tau}-\partial_{\sigma} \mathcal{C}^{\sigma}\right) \\
\left(F_{\mathcal{C}}\right)_{\sigma}^{\sigma}=-\mathcal{C}^{n} \partial_{n}-\frac{1}{2}\left(\partial_{n} \mathcal{C}^{n}\right)-e^{-1} \mathcal{C}^{n}\left(\partial_{n} e\right)+2 \mathcal{C}-\frac{1}{2}\left(\partial_{\tau} \mathcal{C}^{\tau}-\partial_{\sigma} \mathcal{C}^{\sigma}\right)
\end{gathered}
$$

Although $\delta_{B} \mathcal{O}_{\text {ghost }}$ is non-zero, it does not contribute because the regulator matrix $\mathcal{R}$ is block diagonal in the $\overline{\mathcal{C}}_{n}$ and $\mathcal{C}^{n}$ sectors. It turns out that, even in each sector, only diagonal terms contribute because of the nature of $\mathcal{R}$. Hence, in Eq.(9.67) we display only the diagonal part of $F^{A}{ }_{C}$. Finally, since the contribution from $\mathcal{C}_{\tau}$ turns out to be equal to the contribution from $\mathcal{C}_{\sigma}$, the two $\partial_{\tau} \mathcal{C}^{\tau}-\partial_{\sigma} \mathcal{C}^{\sigma}$ terms in Eq.(9.67) for $F_{\mathcal{C}}$ cancel. For the rest of this section, we drop these terms.

The final step is the computation of $(\Delta S)_{\text {reg. }}$. Since $\delta_{B} \mathcal{O}$ does not contribute, we may use Eq.(8.56). At this stage, we expand about the classical saddle point, denoted by $\Sigma$, corresponding to the solution to the equations of motion. We set the $\bar{\pi}$ equal to zero and evaluate the $e_{a}{ }^{m}$ as in Eq.(7.43).

To determine the regulators and $F^{A}{ }_{B}$ at $\Sigma$, note that

$$
\left.e\right|_{\Sigma}=\rho
$$

It is useful to express the regulators in terms of symmetric operators $\tilde{\mathcal{R}}$. For $X^{\mu}$,

$$
\left.\left(\mathcal{R}_{X}\right)\right|_{\Sigma}=\rho^{-1}\left(-\partial_{\tau} \partial_{\tau}+\partial_{\sigma} \partial_{\sigma}\right)=\rho^{-1 / 2} \tilde{\mathcal{R}}_{X} \rho^{1 / 2},
$$

where

$$
\tilde{\mathcal{R}}_{X}=\rho^{-1 / 2} \partial^{n} \partial_{n} \rho^{-1 / 2} .
$$

For ghosts,

$$
\left.\mathcal{R}_{\overline{\mathcal{C}}}\right|_{\Sigma}=\rho \tilde{\mathcal{R}}_{\overline{\mathcal{C}}} \rho^{-1},\left.\quad \mathcal{R}_{\mathcal{C}}\right|_{\Sigma}=\rho^{-1} \tilde{\mathcal{R}}_{\mathcal{C}} \rho
$$

where

$$
\tilde{\mathcal{R}}_{\overline{\mathcal{C}}}=-\rho^{1 / 2} \not \partial \rho^{-2} \not \partial \rho^{1 / 2}, \quad \tilde{\mathcal{R}}_{\mathcal{C}}=-\rho^{-1} \not \partial \not \rho^{-1} .
$$


The conjugating factors in Eqs.(9.69) and (9.71) can be commuted past the operators $F_{B}^{A}$ to arrive at the following equivalent form for $(\Delta S)_{\text {reg }}$

$$
\begin{gathered}
(\Delta S)_{\text {reg }}=\left[\left(\tilde{F}_{X}\right)_{\nu}^{\mu}\left(\exp \left(\frac{\tilde{\mathcal{R}}_{X}}{\mathcal{M}^{2}}\right)\right)_{\mu}^{\nu}+\right. \\
\left.\left(\tilde{F}_{\overline{\mathcal{C}}}\right)_{n}^{n}\left(\exp \left(\frac{\tilde{\mathcal{R}}_{\overline{\mathcal{C}}}}{\mathcal{M}^{2}}\right)\right)_{n}^{n}+\left(\tilde{F}_{\mathcal{C}}\right)_{n}^{n}\left(\exp \left(\frac{\tilde{\mathcal{R}}_{\mathcal{C}}}{\mathcal{M}^{2}}\right)\right)_{n}^{n}\right]_{0},
\end{gathered}
$$

where

$$
\begin{gathered}
\left(\tilde{F}_{X}\right)_{\nu}^{\mu}=\delta_{\nu}^{\mu}\left(\mathcal{C}^{n} \partial_{n}+\frac{1}{2}\left(\partial_{n} \mathcal{C}^{n}\right)-\mathcal{C}\right), \\
\left(\tilde{F}_{\overline{\mathcal{C}}}\right)_{\tau}^{\tau}=\left(\tilde{F}_{\overline{\mathcal{C}}}\right)_{\sigma}^{\sigma}=-\mathcal{C}^{n} \partial_{n}-\frac{1}{2}\left(\partial_{n} \mathcal{C}^{n}\right)-\mathcal{C}, \\
\left(\tilde{F}_{\mathcal{C}}\right)_{\tau}^{\tau}=\left(\tilde{F}_{\mathcal{C}}\right)_{\sigma}^{\sigma}=-\mathcal{C}^{n} \partial_{n}-\frac{1}{2}\left(\partial_{n} \mathcal{C}^{n}\right)+2 \mathcal{C} .
\end{gathered}
$$

The $T_{A B}$ matrix has been judiciously chosen so that the violation in the quantum master equation is proportional to $\mathcal{C}$. The coefficient of $\mathcal{C}^{n}$ in Eq.(9.73) vanishes. To see this, let $\psi_{r}$ and $-E_{r}^{2}$ be the eigenfunctions and eigenvalues of any of the $\tilde{\mathcal{R}}$ operators:

$$
\tilde{\mathcal{R}} \psi_{r}=-E_{r}^{2} \psi_{r}
$$

The $\psi_{r}$ can be chosen to be real. The terms in (9.74) involving $\mathcal{C}^{n}$ enter in the combination $\mathcal{C}^{n} \partial_{n}+\frac{1}{2}\left(\partial_{n} \mathcal{C}^{n}\right)$. Such a combination gives a zero contribution to $(\Delta S)_{\text {reg }}$ since

$$
\begin{gathered}
\left(\mathcal{C}^{n} \partial_{n}+\frac{1}{2}\left(\partial_{n} \mathcal{C}^{n}\right)\right)\left(\exp \left(\frac{\tilde{\mathcal{R}}}{\mathcal{M}^{2}}\right)\right)= \\
\int \mathrm{d} \tau \int_{0}^{\pi} \mathrm{d} \sigma \sum_{r} \psi_{r}(\tau, \sigma)\left(\mathcal{C}^{n} \partial_{n}+\frac{1}{2}\left(\partial_{n} \mathcal{C}^{n}\right)\right) \psi_{r}(\tau, \sigma) \exp \left(-\frac{E_{r}^{2}}{\mathcal{M}^{2}}\right)= \\
\frac{1}{2} \int \mathrm{d} \tau \int_{0}^{\pi} \mathrm{d} \sigma \sum_{r} \partial_{n}\left(\psi_{r}^{2}(\tau, \sigma) \mathcal{C}^{n} \exp \left(-\frac{E_{r}^{2}}{\mathcal{M}^{2}}\right)\right) \rightarrow 0
\end{gathered}
$$

where, in the last step, we assume that quantities fall off sufficiently fast at large $\tau$ and obey appropriate boundary conditions at $\sigma=0$ and $\sigma=\pi$. The reader can also verify the absence of a $\mathcal{C}^{n}$ anomaly directly by using the methods in Appendix $\mathrm{C}$.

To evaluate the terms in $(\Delta S)_{\text {reg }}$ proportional to $\mathcal{C}$, let

$$
H_{r} \equiv-\rho^{-(r+1) / 2} \not \partial \rho^{r} \not \partial \rho^{-(r+1) / 2}
$$

The tilde regulators in Eqs.9.70) and (9.72) are

$$
\tilde{\mathcal{R}}_{X}=H_{0}, \quad \tilde{\mathcal{R}}_{\overline{\mathcal{C}}}=H_{-2}, \quad \tilde{\mathcal{R}}_{\mathcal{C}}=H_{1},
$$


except that, for $X^{\mu}, \tilde{\mathcal{R}}_{X}$ acts in a $D$-dimensional space, whereas $H_{0}$ acts in a twodimensional space, since $-\not \partial \not=I_{2} \partial^{n} \partial_{n}=I_{2}\left(-\partial_{\tau} \partial_{\tau}+\partial_{\sigma} \partial_{\sigma}\right)$, where $I_{2}$ denotes the two-dimensional unit matrix. The non-zero terms in $(\Delta S)_{\text {reg }}$ in Eq.(9.73) all involve

$$
\frac{\operatorname{Tr}}{2}\left[\exp \left(\frac{H_{r}}{\mathcal{M}^{2}}\right)\right]_{0} \equiv \int \mathrm{d} \tau \int_{0}^{\pi} \mathrm{d} \sigma \kappa_{r} .
$$

The trace $\operatorname{Tr}$ is over both 2 by 2 gamma space and function space. The coefficients $\kappa_{r}$, which are computed in Appendix C, are

$$
\kappa_{r}=\frac{1}{24 \pi i}(3 r+1) \partial^{n} \partial_{n} \ln (\rho) \quad .
$$

The contributions to the violation of the quantum master equation from the $X^{\mu}$, $\overline{\mathcal{C}}_{n}$, and $\mathcal{C}_{n}$ sectors are respectively $(-1) D \kappa_{0},(-1) 2 \kappa_{-2}$, and $(+2) 2 \kappa_{1}$, where the factors in parentheses are the coefficients of $\mathcal{C}$ in the $\tilde{F}$ in Eq. (9.74). The total anomaly $\mathcal{A}$ on-shell is [210, 121]

$$
\begin{gathered}
(\Delta S)_{\text {reg }}=i \frac{(D-26)}{24 \pi} \int \mathrm{d} \tau \int_{0}^{\pi} \mathrm{d} \sigma \mathcal{C} \partial^{n} \partial_{n} \ln (\rho) \\
=\left.i \frac{(D-26)}{24 \pi} \int \mathrm{d} \tau \int_{0}^{\pi} \mathrm{d} \sigma \mathcal{C} \partial^{n} \partial_{n} \ln (e)\right|_{\Sigma} .
\end{gathered}
$$

It is absent when $D=26$. For $D \neq 26$ no local counter term $\Omega_{1}$ can be added to cancel the violation of the quantum master equation via Eq.(8.39) and the theory is anomalous. 


\section{Brief Discussion of Other Topics}

The following are discussed in this section: applications to global symmetries, a geometric interpretation of the field-antifield formalism, locality, cohomology, the equivalence of lagrangian and hamiltonian approaches, unitarity, the antibracket formalism in a general coordinate system, the $D=26$ closed bosonic string field theory, and the extended formalism for anomalous gauge theories. One topic not addressed is the anti-BRST symmetry [69, 202, 3, 32, 20, 135, 137, 160, 12, 155, 164].

\subsection{Applications to Global Symmetries}

Certain models with continuous rigid symmetries share some of the characteristics of gauge theories, such as the closure only on-shell of the commutator algebra and the presence of field-dependent structure constants. Global supersymmetric theories without auxiliary fields and models employing non-linear realizations of rigid symmetries are often examples of algebras that do not close off-shell. The antibracket formalism can be used to assist in the analysis of such theories [33, 159]. Even though, in the rigid-symmetry case, the parameters $\varepsilon^{\alpha}$ in the transformation law in Eq.(2.1) are not functions of the space-time variable $x$, there is still the notion of a symmetry structure. In other words, the analogs of the structure equations in Sect. 2, such as the Noether identity, the Jacobi identity, etc., still exist. There are two differences for the globally symmetric case: (a) everywhere $\varepsilon^{\alpha}$ appears, it is a constant and (b) the compact notation for Greek indices, associated with gauge transformations, involves a discrete sum but not an integral over space-time. For Latin indices, associated with the $\phi^{i}$, repeated indices still indicate a space-time integral. Taking into account the above two differences, the equations in Sect. 2 hold for the globally symmetric case.

The development of an antibracket-like formalism proceeds as in Sect. 1 . Since the gauge parameters $\varepsilon^{\alpha}$ are not functions of the space-time variable $x$, one introduces constant ghosts $\mathcal{C}^{\alpha}$. The antifields $\phi_{i}^{*}$ for the original fields $\phi^{i}$ are space-time functions, but the antifields for ghosts are constants. Grassmann statistics and ghost numbers are assigned as in the gauge-theory case. In the antibracket and elsewhere, functional derivatives with respect to ghosts and antifields of ghosts are replaced by ordinary partial derivatives. The proper solution $S$ in Eq.(4.29) is a generating functional for the structure tensors. The structure equations are encoded in the classical master equation $(S, S)=0$. Of course, since global symmetries do not affect the rank of the hessian of $S$ at a stationary point, the concept of properness has little meaning: If one wants to treat all global symmetries via an antibracket-like formalism, one should proceed by mimicking the gauge case.

Since global symmetries do not upset the development of perturbation theory, no 
gauge-fixing procedure via a fermion $\Psi$ is implemented. In the quantum theory, the ghosts are only technical tools. They should not be considered as quantum fields. Antifields are still interpreted as sources for rigid symmetries. To perform standard perturbation theory, antifields can be set to zero. Alternatively, one can differentiate with respect to antifields before setting them to zero to obtain global Ward identities. A third approach is to introduce sources $J$ for fields via Eq.(8.14), retain antifields, and construct the effective action $\Gamma$ as described in Sect. 8.4. Anomalous violations of global symmetries can be analyzed by searching for violations of the Zinn-Justin equation $(\Gamma, \Gamma)_{c}=0$ (see Eq.(8.21)). Examples of anomalous global symmetries are the axial vector currents of massless four-dimensional QCD. An application to the $D=4$ supersymmetric Wess-Zumino model is given in ref. [159].

\subsection{A Geometric Interpretation}

This subsection discusses a geometric interpretation of the field-antifield formalism, as presented by E. Witten [273]. See also refs. [175, 155, 21, 218, 176].

The geometric intepretation is made clearer if we first assume that no fermionic fields are present. Let $\mathcal{M}$ denote the manifold of infinite-dimensional function space. The classical fields $\Phi^{A}$ of the theory are local coordinates for $\mathcal{M}$. Then, $\frac{\partial}{\partial \Phi^{A}}$ is a local basis for the tangent space $\mathcal{T} \mathcal{M}$ of vector fields. Likewise, $d \Phi^{A}$ is a basis for the cotangent space $\mathcal{T}^{*} \mathcal{M}$ consisting of differential forms. There exists a natural quadratic form on $\mathcal{T} \mathcal{M} \oplus \mathcal{T}^{*} \mathcal{M}$ given by $\left\langle d \Phi^{A}, \frac{\partial}{\partial \Phi^{B}}\right\rangle=\delta_{B}^{A},\left\langle d \Phi^{A}, d \Phi^{B}\right\rangle=0$, $\left\langle\frac{\partial}{\partial \Phi^{A}}, \frac{\partial}{\partial \Phi^{B}}\right\rangle=0$. Introduce, in an ad hoc manner, two quantities $z^{A}$ and $w_{A}$ and associate $z^{A}$ with $d \Phi^{A}$ and $w_{A}$ with $\frac{\partial}{\partial \Phi^{A}}$, i.e.,

$$
z^{A} \leftrightarrow d \Phi^{A}, \quad w_{A} \leftrightarrow \frac{\partial}{\partial \Phi^{A}} .
$$

Consider the Clifford algebra for $z^{A}$ and $w_{A}$ determined by the quadratic form $\langle$,$\rangle ,$ namely

$$
\left\{z^{A}, w_{B}\right\}=\delta_{B}^{A}, \quad\left\{z^{A}, z^{B}\right\}=0, \quad\left\{w_{A}, w_{B}\right\}=0 \quad,
$$

where $\{$,$\} denotes the anticommutator: \{x, y\}=x y+y x$. A possible representation of the Clifford algebra regards the $z^{A}$ as creation operators and the $w_{A}$ as destruction operators. Then, the most general state at a point $\Phi$ on the manifold is created by $\Omega(\Phi, z)=\Omega_{0}(\Phi)+\Omega_{A}(\Phi) z^{A}+\frac{1}{2} \Omega_{A B}(\Phi) z^{B} z^{A}+\ldots$ acting on a Fock-space vacuum $|0\rangle$, defined by $w_{A}|0\rangle=0$ for all $A$, i. e., it is annihilated by all the $w_{A}$. Representing the $w_{A}$ as $\frac{\partial}{\partial z^{A}},|0\rangle$ can be taken to be 1 when considered as a function of the $z^{A}$. With the association $z^{A} \leftrightarrow d \Phi^{A}$, one sees that $\Omega$ is equivalent to an element of the exterior algebra of differential forms on $\mathcal{M}$, in which differential forms are multiplied 
by using the wedge product $\wedge$. When supplemented with the exterior derivative $d$, this structure becomes the de Rham complex [107, 96]. In summary, one has an irreducible representation of the Clifford algebra in Eq.(10.2) at each point of the manifold.

The Clifford algebra in (10.2) is symmetrical in its treatment of the elements $z$ and $w$. Hence, one can reverse the above viewpoint and regard the $w_{A}$ as creation operators and the $z^{A}$ as annihilation operators. In this picture, let us identify the antifields $\Phi_{A}^{*}$ of the antibracket formalism with the vector-field-like objects $w_{A}$. The most general state at a point $\Phi$ is created by

$$
F\left[\Phi, \Phi^{*}\right]=F_{0}(\Phi)+F^{A}(\Phi) \Phi_{A}^{*}+\frac{1}{2} F^{A B}(\Phi) \Phi_{B}^{*} \Phi_{A}^{*}+\ldots
$$

acting on a state $|0\rangle^{\prime}$ that is annihilated by all $z^{A}$. In this picture, denoted by $R^{\prime}$ by E. Witten, $z^{A}=\frac{\partial_{r}}{\partial \Phi_{A}^{*}}, w_{A}=\Phi_{A}^{*}$, and $|0\rangle^{\prime}$ can be taken to be 1 when regarded as a function of the $\Phi_{A}^{*}$. Two elements $F$ and $G$ in the form of Eq.(10.3) are multiplied using $\left\{\Phi_{A}^{*}, \Phi_{B}^{*}\right\}=0$. Exploiting the association $d \Phi^{A} \rightarrow z^{A}=\frac{\partial_{r}}{\partial \Phi_{A}^{*}}$ in the $R^{\prime}$ picture, the exterior derivative $d \equiv \frac{\partial_{r}}{\partial \Phi^{A}} d \Phi^{A}$ becomes $\frac{\partial_{r}}{\partial \Phi^{A}} \frac{\partial_{r}}{\partial \Phi_{A}^{*}} F=-\Delta F$ when acting on a general functional $F$ of the type in Eq.(10.3). Here, we have used the definition of $\Delta$ given in Eq.(6.7). In computing $\Delta F$, one treats $\Phi^{A}$ and $\Phi_{B}^{*}$ as independent variables. Because the exterior derivative is nilpotent, $\Delta$ satisfies $\Delta^{2}=0$. In short, one arrives at a dual picture of the de Rham complex. It is isomorphic to the standard de Rham complex but not in a natural way because there is no preferred manner of associating the above two Fock-space vacuums $|0\rangle$ and $|0\rangle^{\prime}$. The state $|0\rangle^{\prime}$ of the $R^{\prime}$ picture is represented as $\left(f_{12 \ldots}\right) \prod_{A} z^{A}$ in the first picture, where $f_{12 \ldots}$ is arbitrary. A natural choice does exist if $\mathcal{M}$ is endowed with a measure $d \mu=\left(\mu_{12 \ldots}\right) d \Phi^{1} \wedge d \Phi^{2} \wedge \ldots$ Then, one can take $f=\mu$.

If fermionic fields are present, $\mathcal{M}$ is a supermanifold. Then, $\{$,$\} appears as a$ graded commutator: $\{x, y\}=x y-(-1)^{\epsilon_{x} \epsilon_{y}} y x$. Note that $\epsilon\left(z^{A}\right)=\epsilon_{A}+1=\epsilon\left(w_{A}\right)$, so that $z^{A}$ and $w_{A}$ have the opposite statistics of $\Phi^{A}$. For the bosonic case, $\epsilon_{A}=0$ for all $A$, and $\{$,$\} becomes the usual anticommutator.$

In the $R^{\prime}$ picture, the Clifford algebra in Eq.(10.2) is satisfied when

$$
w_{A}=\Phi_{A}^{*}, \quad z^{A}=(-1)^{\epsilon_{A}} \frac{\partial_{r}}{\partial \Phi_{A}^{*}} .
$$

Then, $-d$ becomes, when acting on $F$ of Eq.(10.3),

$$
(-1)^{\epsilon_{A}+1} \frac{\partial_{r}}{\partial \Phi^{A}} \frac{\partial_{r}}{\partial \Phi_{A}^{*}} F=\Delta F
$$

The nilpotent operator $\Delta$ of the field-antifield formalism is identified with minus the exterior derivative. 
For elements $F$ and $G$ in Eq.(10.3), the antibracket ( , ) is defined by $\left(F\left[\Phi, \Phi^{*}\right], G\left[\Phi, \Phi^{*}\right]\right)$ $\equiv \frac{\partial_{r} F}{\partial \Phi^{A}} \frac{\partial_{l} G}{\partial \Phi_{A}^{*}}-\frac{\partial_{r} F}{\partial \Phi_{A}^{*}} \frac{\partial_{l} G}{\partial \Phi^{A}}$. Using $\Delta$, the antibracket can be expressed as

$$
\Delta(F G)-F \Delta(G)-(-1)^{\epsilon_{G}} \Delta(F) G=(-1)^{\epsilon_{G}}\left(F\left[\Phi, \Phi^{*}\right], G\left[\Phi, \Phi^{*}\right]\right) .
$$

Eq.(10.6) shows that the antibracket is the obstruction of $\Delta$ to be a derivation from the right. Once $\Delta$ has been defined, one can take the left-hand side of Eq.(10.6) to be the definition of the antibracket after multiplying by $(-1)^{\epsilon_{G}}$.

Summarizing, one has the following analogy. If one thinks of function space as a supermanifold, then antifields are the basis vectors in the $R^{\prime}$ picture of the de Rham complex. The operator $\Delta$ is the analog of the exterior derivative. The antibracket is the obstruction to $\Delta$ for it to be a derivation. Interestingly, the quantum master equation

$$
\frac{1}{2}(W, W)-i \hbar \Delta W=0
$$

has the same form as the equation of motion for a Chern-Simons theory [273]. The analog of a gauge transformation in Chern-Simons theory is a quantum BRST transformation [186] in the antibracket formalism. It is not always too easy to obtain solutions $W$ to the quantum master equation. In some sense, finding an appropriate $W$ is equivalent to obtaining the correct measure, i.e., specifying $\mu$.

\subsection{Locality}

An important but technical aspect of quantum field theories is locality. Here, we study this issue in the antibracket formalism [154, 157, 206, 132, 11, 257]. In going from the classical action $S_{0}$ to the proper solution $S$ and to the quantum action $W$, lagrangian terms are added. In a theory defined by a local classical action, the question is whether these terms are also local. Local interactions involve fields and derivatives, up to a finite order, of fields multiplied at the same space-time point. Nonlocal terms are likely to lead to difficulties such as non-renormalizability, nonunitarity or violations of causality.

The discussion of the gauge structure algebra in Sect. 2 used extensively the consequences of the regularity condition in Eq.(2.11). An examination of the proof of Eq.(2.12) reveals that certain operators need to be inverted so that nonlocal effects are possible. Indeed, it is easy to find a $\lambda^{i}$ so that the solution to $S_{0, i} \lambda^{i}=0$ in Eq.(2.12) involves a nonlocal operator $T^{j i}$ or a function $\lambda^{\prime \alpha_{0}}$ that does not fall off fast at large space-time distances. Nonlocality often occurs when the quantity of interest vanishes because it is an integral of a total derivative. As an example, consider $n$ free quantum mechanical particles governed by the action $S_{0}=\frac{1}{2} \int \mathrm{d} t \dot{q}^{i} \dot{q}_{i}$. Note that $S_{0, i}=-\ddot{q}_{i}$. 
Let $\lambda^{i}=\dot{q}^{i}$. Then, $S_{0, i} \lambda^{i}=-\frac{1}{2} \int \mathrm{d} t \frac{d}{d t}\left(\dot{q}^{i} \dot{q}_{i}\right) \rightarrow 0$. The solution in Eq.(2.12) is $\lambda^{\prime \alpha_{0}}=0$ and

$$
T^{j i}\left(t^{\prime}, t\right)=\left\{\begin{array}{rl}
\frac{\delta^{j i}}{2}, & \text { for } t>t^{\prime}, \\
-\frac{\delta^{j i}}{2}, & \text { for } t<t^{\prime},
\end{array},\right.
$$

since $\lambda^{i}(t)=\int \mathrm{d} t^{\prime} S_{0, j}\left(t^{\prime}\right) T^{j i}\left(t^{\prime}, t\right)$. The trivial gauge transformation governed by $T^{j i}\left(t^{\prime}, t\right)$ obeys the correct symmetry property $T^{i j}\left(t, t^{\prime}\right)=-T^{j i}\left(t^{\prime}, t\right)$.

Hence, an important concept is local completeness [154]. Local completeness holds, when solutions to equations, such as in Eqs.(2.12), (2.17) and (2.19), can be satisfied for local functionals or more precisely, in non-integrated versions. The difficulty is that sometimes these equations are valid due to total derivatives.

In principle, it is possible that the gauge structure tensors involve nonlocal operators. This issue has been analyzed in refs. [92, 154, 132]. Given the locality of $S_{0}$ and that the gauge generators $R_{\alpha}^{i}$ are local operators, then the proper solution $S$ of the classical master equation is local. Reference [154] used cohomological arguments to obtain this result. The gauge-fixed classical action $S_{\Psi}$ is then guaranteed to be local if the gauge-fixing fermion $\Psi$ is. Under these conditions, the classical BRST operators $\delta_{B}$ and $\delta_{B_{\Psi}}$ produce local variations. The question of quantum locality is more involved. Since this must be analyzed on a case by case basis, no general statements about the locality of $W$ can be made.

\subsection{Cohomological Aspects}

In this section, we introduce the concept of cohomology. Cohomological methods have been used to obtain certain general results [151, 103, 105, 159, 13, 11, 257, 253, for the field-antifield formalism. For example, the existence proof of the proper solution is based on these methods [105]. This section is intended to assist the reader in understanding such research. Because these methods have been reviewed in refs. [152, 157, 253], our discussion is brief.

Consider a series of spaces $F_{k}$. The integer $k$ labels different grading levels. If $\alpha_{k} \in F_{k}$ then $\alpha_{k}$ is said to have grading $k$. Let $\delta$ be a nilpotent map from one space to a successive space

$$
\ldots \longrightarrow F_{k-1} \stackrel{\delta}{\longrightarrow} F_{k} \stackrel{\delta}{\longrightarrow} F_{k+1} \longrightarrow \ldots \quad .
$$

Nilpotency means that

$$
\delta^{2}=\delta \delta=0 \quad .
$$

One can think of $\delta$ as carrying a grading of 1 . An element $\alpha_{k}$ in $F_{k}$ is said to be closed if $\delta \alpha_{k}=0$. The kernel of $\delta$ for the $k$ th space, $\operatorname{Ker}_{k} \delta$, consists of the set of 
closed elements of $F_{k}$, i.e.,

$$
\operatorname{Ker}_{k} \delta=\left\{\alpha_{k} \mid \alpha_{k} \in F_{k}, \delta \alpha_{k}=0\right\}
$$

An element $\alpha$ is said to be exact if it is expressible as $\alpha=\delta \beta$. The image of $\delta$ in the $k$ th space, $\operatorname{Im}_{k} \delta$, are the exact elements of $F_{k}$, i.e.,

$$
\operatorname{Im}_{k} \delta=\left\{\alpha_{k} \mid \alpha_{k}=\delta \beta_{k-1}, \text { for some } \beta_{k-1} \in F_{k-1}\right\}
$$

Due to the nilpotency of $\delta$, an exact element is automatically closed, so that $\operatorname{Im}_{k} \delta \subset$ $\operatorname{Ker}_{k} \delta$. Consider the equivalence relation $\underset{k}{\sim}$ that identifies two elements of $F_{k}$ if their difference is exact:

$$
\alpha_{k} \tilde{k} \alpha_{k}^{\prime} \text { if there is a } \beta_{k-1} \in F_{k-1} \text { such that } \alpha_{k}-\alpha_{k}^{\prime}=\delta \beta_{k-1} \text {. }
$$

Define $H_{k}(\delta)$ to be the set of elements of $\operatorname{Ker}_{k} \delta$ modulo the equivalence relation $\underset{k}{\sim}$ :

$$
H_{k}(\delta)=\operatorname{Ker}_{k} \delta / \operatorname{Im}_{k} \delta
$$

The cohomology $H_{k}(\delta)$ is equivalent to the elements in $F_{k}$ that are closed but not exact.

A standard example is the de Rham cohomology on an $n$-dimensional manifold $\mathcal{M}$. The spaces $F_{k}$ consist of the differential forms of order $k$ on $\mathcal{M}$ and $\delta$ is the exterior derivative $d$. The dimension of $H_{k}(d)$ is the $k$ th Betti number for $\mathcal{M}$. In this example, more structure can be defined. Differential forms can be multiplied using the wedge product $\wedge$. One can add differential forms so that the formal sum of the $F_{k}$ spaces constitutes an algebra. The exterior derivative respects addition: $d(\alpha+\beta)=d \alpha+d \beta$, and it is a graded derivation from the left of the wedge product: $d\left(\alpha_{j} \wedge \beta_{k}\right)=d \alpha_{j} \wedge$ $\beta_{k}+(-1)^{j} \alpha_{j} \wedge d \beta_{k}$. In applications within the antibracket formalism $\delta$ has these properties, except, with our conventions, $\delta$ is a graded derivation from the right: $\delta(\alpha \beta)=\alpha \delta \beta+(-1)^{\epsilon_{\beta}}(\delta \alpha) \beta$, where multiplication is denoted by juxtaposition of elements.

Cohomological methods can be powerful. However, they often involve subtle issues so that one must proceed with strict rigor. The question of whether a closed element is expressible as $\delta$ ( of something ) often involves global issues; usually, it can be done "locally". Hence, if one is not careful, one can miscalculate the cohomology. In regard to the antibracket formalism, the pitfalls are more severe: The spaces $F_{k}$ are almost always infinite dimensional, and, in quantizing the system, the multiplication operation becomes singular. Furthermore, an ambiguity concerning the issue of locality in Sect. 10.3 enters: one needs to decide whether local or non-local functionals are permitted. 
One cohomology in the antibracket formalism uses the classical BRST operator $\delta_{B}$ for $\delta$. The spaces $F_{k}$ consist of smooth functionals of fields with ghost number $k$. Note that $k$ ranges over all integers, both positive, negative and zero. Functionals form an algebra since they can be added and multiplied. Furthermore, $\delta_{B}$ satisfies the correct properties: it is nilpotent and is a graded derivation from the right. The cohomology $H_{k}\left(\delta_{B}\right)$ is the classical space of observables in the sector with ghost number $k$.

For proving certain results, two other cohomologies are useful. The first uses the Koszul-Tate differential $\delta_{k t}$ [179, 58, 240]. Let $G_{+}$be the condition of setting all ghost fields to zero:

$$
G_{+} \equiv\left\{\Phi^{A}=0 \mid \operatorname{gh}\left[\Phi^{A}\right] \geq 1\right\} .
$$

Then, the Koszul-Tate differential is defined by

$$
\left.\delta_{k t} X \equiv \delta_{B} X\right|_{G_{+}},
$$

where $\delta_{B} X=(X, S)$. When acting on fields (and ghosts), $\delta_{k t}$ produces zero

$$
\delta_{k t} \Phi^{A}=\left.\frac{\partial_{l} S}{\partial \Phi_{A}^{*}}\right|_{G_{+}}=0
$$

since $\delta_{k t} \Phi^{A}$, having ghost number greater than one, must be proportional to ghost fields. Hence, the interesting action is on antifields:

$$
\delta_{k t} \Phi_{A}^{*}=\left.(-1)^{\epsilon_{A}+1} \frac{\partial_{r} S}{\partial \Phi^{A}}\right|_{G_{+}} .
$$

To check the nilpotency of $\delta_{k t}$, it suffices to compute $\delta_{k t}^{2} \Phi_{A}^{*}$. One finds

$$
\delta_{k t}^{2} \Phi_{A}^{*}=\left.(-1)^{\epsilon_{A}+\epsilon_{B}}\left(\left(\frac{\partial_{r} \partial_{r} S}{\partial \Phi_{B}^{*} \partial \Phi^{A}}\right)\left(\frac{\partial_{r} S}{\partial \Phi^{B}}\right)\right)\right|_{G_{+}},
$$

which can be manipulated to give

$$
\delta_{k t}^{2} \Phi_{A}^{*}=\left.(-1)^{\epsilon_{B}}\left(\frac{\partial_{r}}{\partial \Phi^{A}}\left(\frac{\partial_{r} S}{\partial \Phi_{B}^{*}} \frac{\partial_{r} S}{\partial \Phi^{B}}\right)-\frac{\partial_{r} S}{\partial \Phi_{B}^{*}} \frac{\partial_{r} \partial_{r} S}{\partial \Phi^{A} \partial \Phi^{B}}\right)\right|_{G_{+}},
$$

which, using Eqs.(14.16), (A.4) and (10.16), produces

$$
=-\left.\frac{1}{2}\left(\frac{\partial_{r}(S, S)}{\partial \Phi^{A}}\right)\right|_{G_{+}}=0 .
$$

The Koszul-Tate differential is zero because $S$ satisfies the classical master equation. Because $\delta_{k t}$ is constructed using the BRST operator it is a graded derivation, i.e.,

$$
\delta_{k t}(X Y)=X \delta_{k t}(Y)+(-1)^{\epsilon_{Y}} \delta_{k t}(X) Y .
$$


The action of $\delta_{k t}$ for the antifields with ghosts numbers $-1,-2$ and -3 is respectively

$$
\begin{gathered}
\delta_{k t} \phi_{i}^{*}=(-1)^{\epsilon_{i}+1} S_{0, i} \\
\delta_{k t} \mathcal{C}_{\alpha}^{*}=(-1)^{\epsilon_{\alpha}} \phi_{i}^{*} R_{\alpha}^{i} \\
\delta_{k t} \mathcal{C}_{1 \alpha_{1}}^{*}=(-1)^{\epsilon_{\alpha_{1}}+1}\left(\mathcal{C}_{\alpha}^{*} R_{1 \alpha_{1}}^{\alpha}+\frac{1}{2}(-1)^{\epsilon_{i}} \phi_{i}^{*} \phi_{j}^{*} V_{1 \alpha_{1}}^{j i}\right),
\end{gathered}
$$

where the tensors $R$ and $V$ are given in Sect. 2.

An important result is that $H_{k}\left(\delta_{k t}\right)=\emptyset$ for $k \leq-1$, where $\emptyset$ denotes the empty set 105, 152. Let us formally verify this for $k=-1$ and $k=-2$. One can set terms involving ghosts to zero because they either transform to zero or are set to zero. The most general element $\alpha_{-1}$, with ghost number -1 and constructed from antifields, is

$$
\alpha_{-1}=\phi_{i}^{*} \lambda^{i}
$$

where $\lambda^{i}$ are functionals of the $\phi$, with $\epsilon\left(\lambda^{i}\right)=\epsilon_{i}$ so that $\epsilon\left(\alpha_{-1}\right)=1$. Since $\delta_{k t} \alpha_{-1}=$ $-S_{0, i} \lambda^{i}, \alpha_{-1}$ is closed if $S_{0, i} \lambda^{i}=0$. Accordingly, $\lambda^{i}$ must be expressible as in Eq.(2.12), so that

$$
\alpha_{-1}=\phi_{i}^{*} R_{\alpha}^{i} \lambda^{\prime \alpha}+\phi_{i}^{*} S_{0, j} T^{j i}
$$

This is the most general form for a closed element with ghost number -1 . The key question is whether $\alpha_{-1}$ is exact, i.e., $\alpha_{-1} \stackrel{?}{=} \delta_{k t} \beta_{-2}$. Let

$$
\beta_{-2}=\mathcal{C}_{\alpha}^{*} \lambda^{\prime \alpha}+(-1)^{\epsilon_{i}+1} \frac{1}{2} \phi_{i}^{*} \phi_{j}^{*} T^{j i}
$$

Then, a short computation reveals that $\delta_{k t} \beta_{-2}=\alpha_{-1}$. In the $k=-1$ sector, there are no closed elements that are not exact, so that the cohomology is trivial $H_{-1}\left(\delta_{k t}\right)=\emptyset$.

In the $k=-2$ sector, the most general element is

$$
\alpha_{-2}=\mathcal{C}_{\alpha}^{*} \lambda^{\alpha}+(-1)^{\epsilon_{i}} \frac{1}{2} \phi_{i}^{*} \phi_{j}^{*} M_{0}^{j i},
$$

where $\epsilon\left(\lambda^{\alpha}\right)=\epsilon_{\alpha}$ and $\epsilon\left(M_{0}^{j i}\right)=\epsilon_{i}+\epsilon_{j}$ (mode 2), so that $\epsilon\left(\alpha_{-2}\right)=0$. The functional $M_{0}^{j i}$ obeys $M_{0}^{j i}=(-1)^{\epsilon_{i} \epsilon_{j}+1} M_{0}^{i j}$. A computation reveals that

$$
\delta_{k t} \alpha_{-2}=\phi_{i}^{*}\left(R_{\alpha}^{i} \lambda^{\alpha}-S_{0, j} M_{0}^{j i}\right)
$$

so that

$$
R_{\alpha}^{i} \lambda^{\alpha}-S_{0, j} M_{0}^{j i}=0
$$

if $\alpha_{-2}$ is to be closed. Is $\alpha_{-2} \stackrel{?}{=} \delta_{k t} \beta_{-3}$. It takes a little work to show that $\alpha_{-2}$ is exact. For $\alpha_{-2}$ to be closed, $\lambda^{\alpha}$ must obey Eq.(2.12). Substituting the solution of Eq.(2.12) for $\lambda^{\alpha}$ into Eq. (10.20) gives

$$
R_{\alpha}^{i}\left(R_{1 \alpha_{1}}^{\alpha} \lambda^{\prime \alpha_{1}}+S_{0, j} T_{0}^{j \alpha}\right)-S_{0, j} M_{0}^{j i}=0,
$$


or, using Eq.(2.19),

$$
S_{0, j}\left(V_{1 \alpha_{1}}^{j i} \lambda^{\prime \alpha_{1}}+(-1)^{\epsilon_{j}\left(\epsilon_{i}+\epsilon_{\alpha}\right)} R_{\alpha}^{i} T_{0}^{j \alpha}-M_{0}^{j i}\right)=0 .
$$

The general solution of this equation is

$$
V_{1 \alpha_{1}}^{j i} \lambda^{\prime \alpha_{1}}+(-1)^{\epsilon_{j}\left(\epsilon_{i}+\epsilon_{\alpha}\right)} R_{\alpha}^{i} T_{0}^{j \alpha}-(-1)^{\epsilon_{i} \epsilon_{\alpha}} R_{\alpha}^{j} T_{0}^{i \alpha}-M_{0}^{j i}=S_{0, k} N^{k j i},
$$

where $N^{k j i}$ must be graded antisymmetric in all indices and $\epsilon\left(N^{k j i}\right)=\epsilon_{i}+\epsilon_{j}+\epsilon_{k}$ $(\bmod 2)$. In terms of the above functionals and tensors, let

$$
\beta_{-3}=-\mathcal{C}_{1 \alpha_{1}}^{*} \lambda^{\prime \alpha_{1}}-(-1)^{\epsilon_{\alpha}} \mathcal{C}_{\alpha}^{*} \phi_{j}^{*} T_{0}^{j \alpha}+\frac{1}{6}(-1)^{\epsilon_{j}} \phi_{i}^{*} \phi_{j}^{*} \phi_{k}^{*} N^{k j i} .
$$

Then, a short computation shows that $\alpha_{-2}=\delta_{k t} \beta_{-3}$. Since any closed $k=-2$ element is expressible as an exact form, $H_{-2}\left(\delta_{k t}\right)=\emptyset$.

Notice that the triviality of the Koszul-Tate cohomology $H_{-k}\left(\delta_{k t}\right)=\emptyset$ for $k>0$ reflects the consequences of the regularity condition used in Sect. 2. Plthough the above discussion has been formal, a more rigorous analysis can be given. See refs. 105, 152].

Insight into the physical significance of the Koszul-Tate cohomology is gained by computing $H_{0}\left(\delta_{k t}\right)$. A general closed element of the zero ghost-number sector is a functional $\alpha_{0}$ of the fields $\phi$ since $\delta_{k t} \alpha_{0}(\phi)$ is automatically zero due to $\delta_{k t} \phi^{i}=0$. Let $\beta_{-1}$ be a general element of the -1 ghost sector, i.e, $\beta_{-1}=-\phi_{i}^{*} \lambda^{i}$, where we include a minus sign for convenience, and where the Grassmann nature of $\lambda^{i}$ is $\epsilon\left(\lambda^{i}\right)=\epsilon_{i}$. Apply the Koszul-Tate differential to $\beta_{-1}$ to obtain $\delta_{k t} \beta_{-1}=S_{0, i} \lambda^{i}$. One concludes that if $\alpha_{0}=S_{0, i} \lambda^{i}$ then $\alpha_{0}$ is exact. Therefore $\alpha_{0}$ and $\alpha_{0}{ }^{\prime}$ are not related under the equivalence relation $\underset{0}{\sim}$ if $\alpha_{0}$ and $\alpha_{0}{ }^{\prime}$ differ on the stationary surface $\Sigma$ where the equations of motion $S_{0, i}=0$ hold. Consequently, $H_{0}\left(\delta_{k t}\right)$ corresponds to the set of distinct functions on $\Sigma$. More precisely,

$$
H_{0}\left(\delta_{k t}\right)=\left\{\alpha_{0}(\phi) \mid \alpha_{0} \sim \alpha_{0}^{\prime}, \text { if } \alpha_{0}-\alpha_{0}^{\prime}=S_{0, i} \lambda^{i} \text { for some } \lambda^{i}\right\} .
$$

Suppose that a theory has no gauge invariances. Then the classical observables correspond to functionals taking on distinct values on $\Sigma$. This space is $H_{0}\left(\delta_{k t}\right)$.

If a theory has gauge invariances, then the observables should be the gaugeinvariant elements of $H_{0}\left(\delta_{k t}\right)$. To facilitate the issue of gauge invariance, one introduces the vertical differential $\delta_{g}$ [106, 152]. An alternative name for $\delta_{g}$ is the "exterior derivative along the gauge orbit". It is defined as

$$
\delta_{g} X=\left.\left(\delta_{B} X\right)\right|_{G_{-}}=\left.(X, S)\right|_{G_{-}},
$$

\footnotetext{
${ }^{14}$ If one does not use a proper solution for $S, H_{-k}\left(\delta_{k t}\right)$ can be non-empty for $k>0$.
} 
where $G_{-}$corresponds to the condition of setting antifields to zero and going on-shell with respect to the original fields, i.e.,

$$
G_{-}=\left\{\Phi_{A}^{*}=0, \frac{\partial_{r} S}{\partial \phi^{i}}=0\right\} .
$$

Because $\delta_{g}$ is defined in terms of $\delta_{B}$, it is a derivation from the right:

$$
\delta_{g}(X Y)=X \delta_{g} Y+(-1)^{\epsilon_{y}}\left(\delta_{g} X\right) Y .
$$

Antifields can be ignored in evaluating the vertical differential because they are either set to zero or transformed to zero since

$$
\delta_{g} \Phi_{A}^{*}=-\left.\left(\frac{\partial_{l} S}{\partial \Phi^{A}}\right)\right|_{G_{-}}=0
$$

which follows from ghost number considerations or Eq.(10.23). On fields, one has

$$
\delta_{g} \Phi^{A}=\left.\left(\frac{\partial_{l} S}{\partial \Phi_{A}^{*}}\right)\right|_{G_{-}} .
$$

To check the nilpotency of $\delta_{g}$, one only needs to check that $\delta_{g} \delta_{g} \Phi^{A}=0$. A straightforward calculation gives

$$
\begin{gathered}
\delta_{g}^{2} \Phi^{A}=\left.\left(\frac{\partial_{r} \partial_{l} S}{\partial \Phi^{B} \partial \Phi_{A}^{*}} \frac{\partial_{l} S}{\partial \Phi_{B}^{*}}\right)\right|_{G_{-}} \\
=\left.\left(\frac{\partial_{l}}{\partial \Phi_{A}^{*}}\left(\frac{\partial_{r} S}{\partial \Phi^{B}} \frac{\partial_{l} S}{\partial \Phi_{B}^{*}}\right)-(-1)^{\left(\epsilon_{A}+1\right) \epsilon_{B}} \frac{\partial_{r} S}{\partial \Phi^{B}} \frac{\partial_{l} \partial_{l} S}{\partial \Phi_{A}^{*} \partial \Phi_{B}^{*}}\right)\right|_{G_{-}} \\
=\left.\frac{1}{2}\left(\frac{\partial_{l}(S, S)}{\partial \Phi_{A}^{*}}\right)\right|_{G_{-}}=0,
\end{gathered}
$$

where Eqs.(4.16) and (10.25) have been used. As a consequence of nilpotency, a cohomology with respect to $\delta_{g}$ can be defined.

The physical relevance of $\delta_{g}$ can be seen by computing $H_{0}\left(\delta_{g}\right)$. The action of $\delta_{g}$ on the original fields $\phi^{i}$ is

$$
\delta_{g} \phi^{i}=R_{\alpha}^{i} \mathcal{C}^{\alpha} .
$$

Without loss of generality, a functional $\alpha_{0}$ with ghost number 0 can be taken to be a functional of the $\phi^{i}$ only. Such a functional is closed if $\delta_{g} \alpha_{0}(\phi)=0$. Using Eq. (10.27), one finds

$$
\delta_{g} \alpha_{0}=\alpha_{0, i} R_{\alpha}^{i} \mathcal{C}^{\alpha}=0 \Rightarrow \alpha_{0} \text { is gauge invariant }
$$


Since any functional $\beta_{-1}$ with ghost number -1 is annihilated by $\delta_{g}$, a closed $\alpha_{0}$ cannot be exact: $\alpha_{0} \neq \delta_{g} \beta_{-1}$. The conclusion is that

$$
H_{0}\left(\delta_{g}\right)=\text { the set of gauge-invariant functionals } .
$$

With the above insights, one realizes that observables should roughly correspond to $H_{0}\left(\delta_{k t}\right) \cap H_{0}\left(\delta_{g}\right)$. However, a difficulty arises. The intersection $H_{0}\left(\delta_{k t}\right) \cap H_{0}\left(\delta_{g}\right)$ does not make sense unless $\delta_{k t} \delta_{g}+\delta_{g} \delta_{k t}=0$. The situation is analogous to the one in quantum mechanics where one seeks a state that is simultaneously the eigenvector of two different operators. Such a state is possible if the two operators commute. Because of the graded nature of $\delta_{k t}$ and $\delta_{g}$, the analog condition is that $\delta_{k t}$ and $\delta_{g}$ anticommute. The difficulty can be posed as a question: Should one take the gaugeinvariant elements of $H_{0}\left(\delta_{k t}\right)$ or should one take the elements of $H_{0}\left(\delta_{g}\right)$ modulo the equivalence relation of Eq.(10.21)? If $\delta_{k t} \delta_{g}+\delta_{g} \delta_{k t}=0$, then the above two procedures yield the same result. In such a case, one can define a nilpotent BRST operator $\delta_{B} \equiv \delta_{k t}+\delta_{g}$, and the observables correspond to the BRST cohomology. Unfortunately, $\delta_{k t} \delta_{g}+\delta_{g} \delta_{k t} \neq 0$ in general. An inspection of $\delta_{B}, \delta_{k t}$ and $\delta_{g}$ reveals that $\delta_{B}=\delta_{k t}+\delta_{g}+$ extra terms. The extra terms render $\delta_{B}$ nilpotent, by compensating for the failure of the anticommutivity of $\delta_{k t}$ and $\delta_{g}$. The BRST operator is the natural extension of $\delta_{k t}+\delta_{g}$. The elements of the cohomology of $\delta_{B}$ are the classical observables [105, 152]. They are the definition of what one means by the "gaugeinvariant functionals on $\Sigma$ ".

When quantum effects are incorporated, the quantum BRST transformation $\delta_{\hat{B}}$ is relevant. As discussed in Sect. 8.1, the quantum observables correspond to the elements of the cohomology of $\delta_{\hat{B}}$.

Because canonical transformations preserve the antibracket, the cohomology of $\delta_{B}$ is independent of the basis, as can be seen as follows. Given a proper solution $S\left[\Phi, \Phi^{*}\right]$ in one (untilde) basis, a solution $\tilde{S}\left[\tilde{\Phi}, \tilde{\Phi}^{*}\right]$ in another (tilde) basis is given by $\widetilde{S}\left[\tilde{\Phi}, \tilde{\Phi}^{*}\right] \equiv S\left[\Phi, \Phi^{*}\right]$. Likewise, given any functional $X\left[\Phi, \Phi^{*}\right]$, one can define a functional $\widetilde{X}$ of tilde fields using $\widetilde{X}\left[\tilde{\Phi}, \tilde{\Phi}^{*}\right] \equiv X\left[\Phi, \Phi^{*}\right]$. The tilde antibracket of $\widetilde{X}$ and $\widetilde{S}$, as a function of tilde fields and antifields, equals $(X, S)$ as a function of untilde fields. Hence, $\widetilde{X}$ is closed if and only if $X$ is, and $\widetilde{X}$ is exact if and only if $X$ is. Consequently, there is an exact isomorphism of the cohomologies.

Since the gauge-fixed BRST transformation $\delta_{B_{\Psi}}$ is not nilpotent, one cannot directly define a cohomology associated with $\delta_{B_{\Psi}}$. However, according to Eq. (6.83), $\delta_{B_{\Psi}}^{2}$ is proportional to the equations of motion of the gauge-fixed action $S_{\Psi}$. Define an equivalence relation, denoted by $\approx$, that equates two quantities if they differ by terms proportional to the equations of motion for $S_{\Psi}$. Then, one has $\delta_{B_{\Psi}}^{2} \approx 0$ and a gauge-fixed BRST cohomology can be defined. What is the relation between $H_{n}\left(\delta_{B_{\Psi}}\right)$ 
and $H_{n}\left(\delta_{B}\right)$ ? The connection is best seen by going to the gauge-fixed basis for $\delta_{B}$. Let

$$
Y\left(\tilde{\Phi}, \tilde{\Phi}^{*}\right)=y(\tilde{\Phi})+y^{A}(\tilde{\Phi}) \tilde{\Phi}_{A}^{*}+\ldots
$$

be the antifield expansion of a functional $Y$ in the gauge-fixed basis $\tilde{\Phi}^{A}$ and $\tilde{\Phi}_{A}^{*}$ of Sect. 6.6. Eqs.(6.79) and (6.84) imply

$$
\delta_{B} Y=\delta_{B_{\Psi}} y-y^{A} \frac{\partial_{l} S_{\Psi}}{\partial \tilde{\Phi}^{A}}+O\left(\tilde{\Phi}^{*}\right),
$$

so that

$$
\left.\left(\delta_{B} Y\right)\right|_{\left\{\tilde{\Phi}^{*}=0\right\}} \approx \delta_{B_{\Psi}} y \quad,
$$

since the last term in Eq.(10.30) is proportional to gauge-fixed equations of motion. Eq.(10.31) implies that if $Y$ is $\delta_{B}$-closed then $y$ is $\delta_{B_{\Psi}}$-closed, and that if $Y$ is $\delta_{B}$-exact then $y$ is $\delta_{B_{\Psi}}$-exact. This is not enough to establish any relation between $H_{n}\left(\delta_{B_{\Psi}}\right)$ and $H_{n}\left(\delta_{B}\right)$. Given a element of $y$ of $H_{n}\left(\delta_{B_{\Psi}}\right)$, one must uniquely construct an element $Y$ of $H_{n}\left(\delta_{B_{\Psi}}\right)$, under the condition that $\left.Y\right|_{\left\{\tilde{\Phi}^{*}=0\right\}}=y$. In other words, one must find the higher-order terms in Eq.(10.29). References [151, 105, 103] succeeded in doing this. For additional discussion, see refs. [157, 253]. The cohomologies governed by $\delta_{B_{\Psi}}$ and $\delta_{B}$ are equivalent.

\subsection{Equivalence with the Hamiltonian BFV Formalism}

Gauge theories can also be analyzed using a hamiltonian formalism. For the generic theory, the Batalin-Fradkin-Vilkovisky (BFV) approach [109, 23, 108, 18, 19] is quite useful. For the simplest theories, such as particle models, Yang-Mills theory, and gravitation, it is not difficult to show that it yields results equivalent to the fieldantifield formulation. Demonstrating the equivalence in general, at the classical level or formally at the quantum level without regularization, has been the subject of the work in refs. [31, 104, 230, 231, 91, 140, 141, 201, 206, 75, ,76].

A review of the BFV hamiltonian formalism is given in ref. [150]. Here, we present only the key ideas. Let $S_{0}$ be the classical action as determined from a lagrangian $L$ by $S_{0}=\int \mathrm{d} t L$. The hamiltonian $H_{S_{0}}$ associated with $S_{0}$ is constructed in the standard manner using

$$
H_{S_{0}}[\phi, \pi] \equiv \dot{\phi}^{i} \pi_{i}-L
$$

\footnotetext{
${ }^{15}$ In this subsection, we use the convention that a field index also represents a spatial position. An index appearing twice represents a sum not only that index but also an integration over space. This is the hamiltonian analog of the compact notation described in Sect. 2.1. The difference, here, is that time is not included as part of the integration.
} 
where a dot over a field indicates a time derivative, where the conjugate momentum of $\phi^{i}$ is $\pi_{i} \equiv \frac{\partial_{l} S_{0}}{\partial \dot{\phi}^{i}}, i=1, \ldots, n$, and where $H_{S_{0}}$ is obtained as a function of the $\phi$ and $\pi$ by solving for $\dot{\phi}$ in terms of the $\pi$ and $\phi$. For some systems, this velocity-momentum inversion process is not possible due to the presence of primary constraints. Even in this case, a hamiltonian $H_{S_{0}}$ can be uniquely constructed on the surface of these primary constraints. We symbolically represent the procedure of obtaining a hamiltonian from an action diagrammatically as

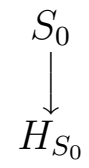

For a wide class of gauge theories, $H_{S_{0}}$ is of the form

$$
H_{S_{0}}=H_{0}[\varphi, \pi]+\lambda^{\alpha} T_{\alpha}[\varphi, \pi] \quad,
$$

where the original $n$ variables $\phi^{i}$ are split into dynamical degrees of freedom $\varphi^{a}, a=$ $1, \ldots, m \leq n$ and Lagrange multipliers $\lambda^{\alpha}$ for the (secondary) constraints $T_{\alpha}$. In Eq.(10.33), $H_{0}$ and $T_{\alpha}$ are functions of the $\varphi$ and their momenta only. The velocities $\dot{\lambda}^{\alpha}$ are usually assumed not to appear in $S_{0}$. This means that the momenta of the $\lambda^{\alpha}$, namely $\pi_{\alpha}$, are primary constraints and do not enter in $H_{S_{0}}$. For example, in a Yang-Mills theory, the hamiltonian density $\mathcal{H}_{0}$ is $\mathcal{H}_{0}=\frac{1}{2} E_{a}^{i} E_{i}^{a}+\frac{1}{4} F_{i j}^{a} F_{a}^{i j}$, where $E_{a}^{i}=-F_{a}^{0 i}=F_{a 0 i}$ are the canonical momenta for the potentials $A_{i}^{a}$, the constraints $T_{\alpha}$ correspond to Gauss's law: $T_{a}=-D_{i a}{ }^{b} E_{b}^{i}$, and the Lagrange multipliers $\lambda^{\alpha}$ are $A_{0}^{a}$.

For simplicity, assume that the constraints $T_{\alpha}$ and the hamiltonian $H_{0}$ are first class, i.e,

$$
\left\{T_{\alpha}, T_{\beta}\right\}_{P B}=T_{\alpha \beta}^{\gamma} T_{\gamma}, \quad\left\{H_{0}, T_{\alpha}\right\}_{P B}=V_{\alpha}^{\beta} T_{\beta},
$$

where $\{,\}_{P B}$ denotes the graded Poisson bracket defined by

$$
\{F, G\}_{P B}=(-1)^{\epsilon_{i}} \frac{\partial_{r} F}{\partial \phi^{i}} \frac{\partial_{l} G}{\partial \pi_{i}}-\frac{\partial_{r} F}{\partial \pi_{i}} \frac{\partial_{l} G}{\partial \phi^{i}} .
$$

Here, the sum over $i$ is such that all fields and momenta are included. If the constraints are second class, Dirac brackets 88 must be used. Note that $\left\{\pi_{i}, \phi^{j}\right\}_{P B}=-\delta_{i}^{j}$.

The BFV program is based on BRST invariance. One introduces ghosts and their conjugate momenta. The ghosts needed correspond to the minimal set, introduced in Eq.(4.1). For the irreducible case, they are the $\mathcal{C}^{\alpha}$. We use $\overline{\mathcal{P}}_{a}$ to denote the momentum associated with a ghost $\mathcal{C}^{a}$, where $a$ is a label that enumerate all ghosts. The Poisson bracket in Eq.(10.35) is then extended to include a sum over ghosts. With these conventions, $\left\{\overline{\mathcal{P}}_{b}, \mathcal{C}^{a}\right\}_{P B}=-\delta_{b}^{a}$. The ghost numbers and statistics of the 
BFV ghosts are the same as in Sect. 4.1. For momenta, gh $\left[\overline{\mathcal{P}}_{a}\right]=-\operatorname{gh}\left[\mathcal{C}^{a}\right]$ and $\epsilon\left(\overline{\mathcal{P}}_{a}\right)=\epsilon\left(\mathcal{C}^{a}\right)$. A canonical generator of the BRST transformations $Q_{B}$ and an extended hamiltonian $H$ are constructed, using the requirement that $Q_{B}$ be nilpotent and that $H$ be BRST invariant:

$$
\left\{Q_{B}, Q_{B}\right\}_{P B}=0, \quad\left\{H, Q_{B}\right\}_{P B}=0
$$

They can be expanded as a power series in ghost fields, for which the first few terms are

$$
H=H_{0}+\mathcal{C}^{\alpha} V_{\alpha}^{\beta} \overline{\mathcal{P}}_{\beta}+\ldots, \quad Q_{B}=\mathcal{C}^{\alpha} T_{\alpha}-\frac{1}{2}(-1)^{\epsilon_{\beta}} \mathcal{C}^{\beta} \mathcal{C}^{\gamma} T_{\gamma \beta}^{\alpha} \overline{\mathcal{P}}_{\alpha}+\ldots .
$$

It turns out that the requirement of $\left\{Q_{B}, Q_{B}\right\}_{P B}=0$ reproduces the relations defining the structure of the gauge algebra at hamiltonian level. In other words, $Q_{B}$ plays a role analogous to the proper solution $S$ of the antibracket formalism.

In this approach, $\mathcal{O}$ is an observable if it is BRST-invariant, i.e., $\left\{\mathcal{O}, Q_{B}\right\}_{P B}=0$. Thus, the hamiltonian is an observable. Two observables $\mathcal{O}_{1}$ and $\mathcal{O}_{2}$ are considered equivalent if $\mathcal{O}_{2}=\mathcal{O}_{1}+\left\{\mathcal{O}^{\prime}, Q_{B}\right\}_{P B}$, for some $\mathcal{O}^{\prime}$. A state $|\psi\rangle$ is called physical if $Q_{B}|\psi\rangle=0$. Two states $\left|\psi_{1}\right\rangle$ and $\left|\psi_{2}\right\rangle$ are considered equivalent if $\left|\psi_{2}\right\rangle=\left|\psi_{1}\right\rangle+$ $Q_{B}\left|\psi^{\prime}\right\rangle$, for some $\left|\psi^{\prime}\right\rangle$.

Given a suitable hamiltonian $H$, a lagrangian can be constructed via

$$
\exp \left(\frac{i}{\hbar} S_{H}[\Phi, \dot{\Phi}]\right)=\int \frac{[\mathrm{d} \Pi]}{2 \pi i \hbar} \exp \left[\frac{i}{\hbar} \int \mathrm{d} t\left(\dot{\Phi}^{i} \Pi_{i}-H[\Phi, \Pi]\right)\right],
$$

where $\Phi$ denotes all degrees of freedom and $\Pi$ denotes the corresponding momenta. We indicate the process of constructing an action $S_{H}$ from a hamiltonian $H$ by the following diagram

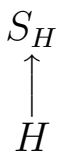

In the $\mathrm{BFV}$ formalism, to obtain a hamiltonian $H_{\Psi}$, which is appropriate for insertion in the functional integral, a fermion $\Psi$ with ghost number minus one is used. As in the antibracket formalism, BRST trivial pairs exist. Given two fields $\Lambda$ and $\Sigma$, and their conjugate momenta, $\overline{\mathcal{P}}_{\Lambda}$ and $\overline{\mathcal{P}}_{\Sigma}$, a term $\int \mathrm{d}^{d-1} x \Sigma \overline{\mathcal{P}}_{\Lambda}$ can be added to $Q_{B}$ without ruining nilpotency. The next step in the BFV program is to introduce additional fields and their momenta and add them as trivial pairs to $Q_{B}$. These fields are the analogs of the auxiliary gauge-fixing fields of Sect. 6.2. They include antighosts, extraghosts, and the Lagrange-multiplier fields of Eq.(6.22). The fermion $\Psi$ in the hamiltonian formulation must satisfy conditions similar to those in Sect. 6.3 
for the $\Psi$ in the antibracket formalism. We denote the BRST charge extended by the inclusion of the additional trivial terms by $Q_{B}^{\mathrm{nm}}$. The hamiltonian $H_{\Psi}$ is given by

$$
H_{\Psi}=H-\left\{\Psi, Q_{B}^{\mathrm{nm}}\right\}_{P B} .
$$

Let $Z_{\Psi}=\int[\mathrm{d} \Phi] \exp \left(\frac{i}{\hbar} S_{H_{\Psi}}\right)$, where $S_{H_{\Psi}}$ is constructed from $H_{\Psi}$ via Eq. (10.38). The Fradkin-Vilkovisky theorem [109] states that $Z_{\Psi}$ is independent of $\Psi$.

The equivalence of the BFV hamiltonian and antibracket methods is established if the remaining leg of the following diagram

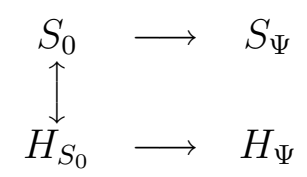

can be completed. In other words, is $S_{H_{\Psi}}$, as constructed from $H_{\Psi}$ via Eq. (10.38), equivalent to $S_{\Psi}$ as obtained from the antibracket formalism? Likewise, is $H_{S_{\Psi}}$, as constructed from $S_{\Psi}$ via Eq. (10.32), equivalent to the BFV hamiltonian $H_{\Psi}$ ? Another question is whether the gauge-fixed BRST charge $Q_{\text {Noether }}$, as constructed from $S_{\Psi}$ using Noether's theorem, coincides with the BRST charge $Q_{B}^{\text {nm }}$ for the BFV formalism. The affirmative answer to the above questions, obtained in refs. [104, 91], implies that construction processes in

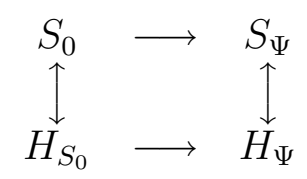

commute to give equivalent results.

One can also ask whether an equivalence occurs before the introduction of gaugefixing and $\Psi$ :

$$
\begin{aligned}
& S \\
& \downarrow \\
& H
\end{aligned}
$$

Clearly, a straightforward correspondence cannot exist because $S$ contains antifields. However, at least for closed irreducible theories, if certain antifields are set to zero and others are identified with ghost momenta, then an equivalence of $H_{S}$, as constructed from $S$ via Eq.(10.32), and the BFV $H$ is achieved [31]. Similar results have been obtained in refs. [230, 91].

If sources for the BRST transformations are included at the hamiltonian level, the above correspondence can be made clearer. Then, the sources in the hamiltonian formulation can be identified with antifields in the antibracket formalism. This method was used in refs. [140, 75, 76] to establish the equivalence in the gauge-fixed basis.

An open problem is to extend all of the above analysis to the quantum case in a rigorous manner. That situation is more difficult due to operator ordering problems and the singular character of field theories. 


\subsection{Unitarity}

The difficulty in proving unitarity in covariant approaches to quantizing gauge theories is due to the presence of ghosts and of unphysical degrees of freedom with negative norms. One often deals with indefinite-metric Hilbert spaces. Unitarity can be spoiled in theories with kinetic energy terms of the wrong sign and/or nonhermitian interaction terms. Wrong sign kinetic energy terms almost always arise in gauge theories with particles of spin one or higher. Due to the sign of the metric component $\eta_{00}$, there are potential difficulties with the temporal components, such as $A_{0}$ in electromagnetism, $A_{0}^{a}$ in Yang-Mills theories, and $g_{0 i}$ in gravity. Faddeev-Popov and other gauge-fixing ghosts enter in loops with the wrong sign, and would lead to a violation of unitarity, if their contributions were considered in isolation.

Let us summarize how unitarity is established in certain covariant quantization procedures. First of all, one needs to assume that there are not any non-hermitian interactions in the original theory and that the spatial components of tensors have the correct sign in kinetic energy terms. In other words, the theory should be "naively" unitary.

The first approach is as follows. In some theories, there exists a unitary gauge, in which it is evident that the unphysical excitations are not present. If one can establish the gauge invariance of the $S$-matrix, then unitarity can be proven by going from a covariant gauge to an unitary one [100]. Unfortunately, this method is only well developed for irreducible theories with closed algebras. For reducible systems, this approach often encounter difficulties, although for some specific examples it has been successfully implemented [116].

Another method for checking unitarity is in perturbation theory via Feynman diagrams 243, 245]. Using the Ward-Takahashi [265, 239] or Slavnov-Taylor identities [241, 233], as well as the Landau-Cutkosky rules [95, one tries to show that contributions from the unphysical polarizations of the classical fields are cancelled by contributions from ghost fields or from other sources.

A third approach proceeds via canonical quantization. The "physical sector" is selected out by imposing some subsidiary conditions that remove negative norm states. The physical sector should be stable under time evolution and should involve a non-negative metric. A well-known example of this approach is the Gupta-Bleuler procedure [53, 144] for quantizing QED. All components of the electromagnetic field $A^{\mu}$ are used; however only states $|\varphi\rangle_{\text {phys }}$ satisfying

$$
\left(\partial_{\mu} A^{\mu}\right)^{+}|\varphi\rangle_{\mathrm{phys}}=0
$$

are considered, where $\left(\partial_{\mu} A^{\mu}\right)^{+}$denotes the positive frequency components of $\partial_{\mu} A^{\mu}$. This condition determines the physical sector $\mathcal{H}_{\text {phys }}$ in the Gupta-Bleuler procedure. 
Unfortunately, when applied to non-abelian Yang-Mills theories, this method fails to preserve $\mathcal{H}_{\text {phys }}$ under time evolution. To quantize covariantly non-abelian gauge theories, refs. [70, 181, 182] proposed

$$
Q_{B}|\varphi\rangle_{\mathrm{phys}}=0
$$

where $Q_{B}$ is the hermitian nilpotent BRST operator. Eq.(10.41) is the basis for BRST quantization. We use $V_{\text {phys }}$ to denote the space of states annihilated by $Q_{B}$. In the BRST approach, the hamiltonian is automatically hermitian so that the $S$-matrix is a unitarity operator in $V_{\text {phys }}$. However, there is a possible difficulty with $V_{\text {phys }}$. Despite the fact that $Q_{B}$ commutes with the hamiltonian, the positive semidefiniteness of the norm of $V_{\text {phys }}$ is not ensured. The question of unitarity in BRST quantization becomes that of proving the positive semidefiniteness of $V_{\text {phys }}$, and must be analyzed model by model.

However, T. Kugo and I. Ojima [181, 182] (see also [196]), obtained criteria under which unitarity does hold. They established a connection with the metric structure of $V_{\text {phys }}$ and the multiplets of the algebra generated by the conserved BRST charge $Q_{B}$ and the conserved ghost number charge $Q_{C}$. With our conventions, $Q_{C}$ is antihermitian: $Q_{C}^{\dagger}=-Q_{C}$. These generators satisfy

$$
\left[Q_{C}, Q_{B}\right]=Q_{B}, \quad\left[Q_{C}, Q_{C}\right]=0, \quad \frac{1}{2}\left\{Q_{B}, Q_{B}\right\}=Q_{B}^{2}=0 \quad .
$$

Three types of multiplets are possible:

(a) "True physical states": BRST singlets with zero ghost number.

(b) Doublets: pairs of BRST singlets related by ghost conjugation.

(c) Quartets: pairs of BRST doublets related by ghost conjugation.

Roughly speaking, ghost conjugation is the operation that interchanges ghosts and antighosts. Under this operation, the sign of the ghost number of a state is flipped. In the next three paragraphs, we explain the classification of the multiplets.

One can choose states to be eigenfunctions of $Q_{c}$. Let $|g\rangle$ be a state with a nonzero ghost number $g$. Then $|g\rangle$ has zero norm since $\left\langle g\left|Q_{c}\right| g\right\rangle=g\langle g \mid g\rangle=-g\langle g \mid g\rangle$, the first equality arising when $Q_{c}$ acts to the right, and the second equality arising when $Q_{c}$ acts to the left. Non-zero matrix elements occur only when bra and ket states have opposite ghost numbers. Under application of $Q_{B}$, the ghost number of

\footnotetext{
${ }^{16}$ We assume there are no anomalies associated with $Q_{B}$ and $Q_{C}$. For the first-quantized string, this is actually not the case for $Q_{C}$, but BRST quantization is still possible 163, 174, 115]. For similar analyses in other models see refs. [59, 42, 48, 60.
} 
a state is increased by one. Such states $|s\rangle=Q_{B}\left|s^{\prime}\right\rangle$ also have null norms since $\langle s \mid s\rangle=\left\langle s^{\prime}\left|Q_{B} Q_{B}\right| s^{\prime}\right\rangle=0$.

Due to the nilpotency of $Q_{B}$, the representations are either BRST singlets or BRST doublets. A BRST singlet $|s\rangle$ satisfies $Q_{B}|s\rangle=0$ and $|s\rangle \neq Q_{B}\left|s^{\prime}\right\rangle$ for any $\left|s^{\prime}\right\rangle$. If $Q_{B}\left|s^{\prime}\right\rangle=|s\rangle \neq 0$, then $\left|s^{\prime}\right\rangle$ is a member of the BRST doublet consisting of $\left|s^{\prime}\right\rangle$ and $|s\rangle$. The upper member of a doublet $|s\rangle$ is annihilated by $Q_{B}$, since $Q_{B}|s\rangle=Q_{B} Q_{B}\left|s^{\prime}\right\rangle=0$, and it carries one unit of ghost number more than $\left|s^{\prime}\right\rangle$ : $Q_{c}|s\rangle=Q_{c}\left|s^{\prime}\right\rangle+1$.

If $|s\rangle$ is a BRST singlet and carries ghost number zero, then it is of type (a). If $|s\rangle$ is a BRST singlet and carries non-zero ghost number $g$, then it is of type (b). Under ghost conjugation, another BRST single with ghost number $-g$ is created, thus forming the pair. If $|s\rangle$ and $\left|s^{\prime}\right\rangle$ constitute a BRST doublet, then ghost conjugation produces another BRST doublet and a type (c) multiplet is obtained.

For an irreducible gauge theory, T. Kugo and I. Ojima in [181, 182] proved that (i) if type (a) states have positive definite norm and (ii) if type (b) states are absent, then quartets only appear in $V_{\text {phys }}$ through zero norm combinations. Consequently, when (i) and (ii) are satisfied, $V_{\text {phys }}$ has a positive semidefinite norm. To obtain a unitary theory, one mods out the null-norm states: Two states are identified if they differ by a null-norm vector. Clearly, null-norm states are identified with the null state. The modding-out procedure automatically restricts states to the zero-ghost number sector, since states with non-zero-ghost number have zero norms. Furthermore, because BRST-trivial states $Q_{B}\left|s^{\prime}\right\rangle$ are null-norm vectors, all that remains after modding out are the non-trivial elements of the $g=0$ BRST cohomology, i.e., states with ghost number zero that are annihilated by $Q_{B}$ and that cannot be expressed as $Q_{B}\left|s^{\prime}\right\rangle$ for any state $\left|s^{\prime}\right\rangle$. This sector is preserved under time evolution because $Q_{B}$ and $Q_{C}$ commute with the hamiltonian.

In the $g=0$ sector, it makes sense to identify null-norm states with the null vector because they decouple from matrix elements involving observables, such as the hamiltonian. Observables $\mathcal{O}$ are BRST-invariant operators: $\left[\mathcal{O}, Q_{B}\right]=0$. If $|t\rangle$ is a BRST-trivial state, so that $|t\rangle=Q_{B}\left|s^{\prime}\right\rangle$, and if $|s\rangle$ is any element of $V_{\text {phys }}$, so that $Q_{B}|s\rangle=0$, then $\langle s|\mathcal{O}| t\rangle=\left\langle s\left|\mathcal{O} Q_{B}\right| s^{\prime}\right\rangle=\left\langle s\left|Q_{B} \mathcal{O}\right| s^{\prime}\right\rangle=0$.

For reducible systems, ghosts for ghosts and extraghosts arise, some of which have zero ghost number. Hence a third condition arises for reducible theories: (iii) a state of $V_{\text {phys }}$ involving ghosts in the $g=0$ sector must be a member of a quartet multiplet. This guarantees that they are null vectors and do not ruin the positive semidefiniteness of $V_{\text {phys }}$.

The above conditions provide criteria for establishing the positivity of the norm and hence unitarity in a covariant formulation. Reference [70, 181, 182] established 
unitarity for Yang-Mills theories by proving (i) and (ii) for this case.

In perturbation theory and in a Fock space representation, A. Slavnov in 234 used (i)-(iii) to obtain simpler criteria. The most important requirements, apart from the positivity of the norm of type (a) states, were that $Q_{B}$ be nilpotent and that it have nontrivial action on all ghost fields or their conjugate momenta. Under these conditions, $V_{\text {phys }}$ has a positive semidefinite norm. Then, S. A. Frolov and A. Slavnov [117] using the hamiltonian BFV-BRST formalism for lagrangians $L$ of the form in Eqs.(10.32) and (10.33), verified the above-mentioned conditions perturbatively. The analysis was simplified because one could use the free BRST charge $Q_{B}^{(0)}$. The criteria became that $Q_{B}^{(0)} Q_{B}^{(0)}=0$ and that $Q_{B}^{(0)}$ have non-trivial action on all ghosts. Given the validity of perturbation theory, their result on the unitarity of a gauge theory holds for the finite reducible case.

S. A. Frolov and A. Slavnov in ref. [118, 235], were able to translate the above program into a lagrangian approach, by using an effective action $A_{\text {eff }}$. The action and BRST charge were perturbatively expanded in a series: $A_{\text {eff }}=A_{\text {eff }}^{(0)}+\ldots$ and $Q_{B}=Q_{B}^{(0)}+\ldots$ The term $A_{\text {eff }}^{(0)}$ was the leading order part of the general gaussian gauge-fixed action $S_{\Psi}$ of the field-antifield formalism presented in Sect. 6.4. Requiring nilpotency and BRST invariance of the action lead to a series of recursion relations for the higher order terms in $A_{\text {eff }}$ and $Q_{B}$. The action $A_{\text {eff }}$, thus obtained, is constructed using unitarity requirements. Finally, when certain conditions on the rank of the gauge generators are imposed, the free BRST charge is seen to act nontrivially on ghosts fields and unphysical polarizations of the classical fields, thereby yielding a unitary theory if the classical gauge-invariant degrees of freedom have a positive norm.

The problem of unitarity in the field-antifield formalism was addressed in 130 , 206, 203, 204. A perturbative solution of the proper solution $S$ was obtained in [130, 206, 132] (see also ref. [13]). Then, a general gaussian gauge-fixing procedure was performed, using a fermion $\Psi$ of the type given in Sects. 6.3 and 6.4. It was shown that BRST invariance of the gauge-fixed action and nilpotency of the gaugefixed BRST transformation lead to the same recursion relations obtained in [118, 235], and that the leading two terms of $S_{\Psi}$ agree with $A_{e f f}$. The conclusion is that the fieldantifield formalism produces an action $S_{\Psi}$ that coincides with $A_{\text {eff }}$ of ref. 1118, 235] obtained by unitarity considerations.

The above approaches to unitarity are formal in that the difficulties with fieldtheoretic infinities are not addressed. The renormalizability or non-renormalizability is not used. To proceed rigorously, one needs to regulate the theory with a cutoff, verify unitarity, and then make sure that unitarity remains as the cutoff is removed. The issue of locality also enters here. For example, it may happen that $A_{e f f}$ or $S$ 
contains non-local terms. This does not necessarily ruin unitarity, but might signal that the theory is non-renormalizable or ill-defined. Studies of unitarity without using perturbation theory for general systems with finite degrees of freedom, such as in quantum mechanics, have been carried out in ref. 194.

\subsection{The Antibracket Formalism in General Coordinates}

The antibracket formalism in a general coordinate system has been developed in refs. 273, 175, 21, 176, 218, 219, 149. A brief overview is given in [157]. This approach sometimes goes by the name covariant formulation of the field-antifield formalism. Possible applications are in mathematics [209, 5, 189, 198, 158 and in string field theory [209, 274, 189, 149, 158, 225, 226].

Consider a supermanifold $\mathcal{M}$ of type $(N, N)$, meaning that there are $N$ bosonic and $N$ fermionic coordinates. Collectively denote these as $z^{a}, a=1, \ldots, 2 N$. In this coordinate system, a local basis for the cotangent space $\mathcal{T}^{*} \mathcal{M}$ consists of the 1-forms $d z^{a}, a=1, \ldots, 2 N$. The Grassmann parity of a differential is the same as that of the corresponding coordinate: $\epsilon\left(d z^{a}\right)=\epsilon\left(z^{a}\right)=\epsilon_{a}$. Introduce an odd two-form $\zeta$, $\epsilon(\zeta)=1$, which is non-degenerate and closed, i.e., $d \zeta=0$. In the local basis, $\zeta$ is expressed as

$$
\zeta=-\frac{1}{2} \zeta_{a b}(z) d z^{b} \wedge d z^{a}=\frac{1}{2} d z^{b} \wedge \zeta_{b a}(z) d z^{a}, \quad \zeta_{a b}=(-1)^{\epsilon_{a} \epsilon_{b}+1} \zeta_{b a} \quad,
$$

where $\epsilon\left(\zeta_{a b}\right)=\epsilon_{a}+\epsilon_{b}+1(\bmod 2)$. Let $\zeta^{a b}$ be the inverse of the matrix $\zeta_{a b}$. It obeys $\zeta^{a b}=(-1)^{\epsilon_{a}+\epsilon_{b}+\epsilon_{a} \epsilon_{b}} \zeta^{b a}$. One then defines the antibracket via Eq. (4.8) but using $\zeta^{a b}(z)$. Alternatively, let $X$ be a function on $\mathcal{M}$. Then one can define a vector field $\overleftarrow{V}_{X}=\frac{\overleftarrow{\partial}}{\partial z^{a}} \zeta^{a b} \frac{\partial_{l} X}{\partial z^{b}}$ that acts from right to left on functions $Y$ of $\mathcal{M}:[Y] \overleftarrow{V}_{X}=\frac{\partial_{r} Y}{\partial z^{a}} \zeta^{a b} \frac{\partial_{l} X}{\partial z^{b}}$. Then the antibracket can be written as

$$
(X, Y)=\zeta\left[\overleftarrow{V}_{X}, \overleftarrow{V}_{Y}\right]=[X] \overleftarrow{V}_{Y}
$$

where the first equality follows from

$$
d z^{b} \wedge d z^{a}\left[\frac{\partial_{r}}{\partial z^{c}}, \frac{\partial_{r}}{\partial z^{d}}\right]=\delta_{c}^{a} \delta_{b}^{d}-(-1)^{\epsilon_{a} \epsilon_{b}} \delta_{c}^{b} \delta_{a}^{d} .
$$

In this way, $\{\mathcal{M}, \zeta\}$ becomes an odd symplectic structure. The antibracket, defined as above, obeys the properties in Eqs.(4.5) and (4.7). It turns out that $d \zeta=0$ is necessary for the Jacobi identity in Eq.(4.5).

For ordinary symplectic manifolds, there exists a natural volume element $d \mu$ obtained by wedging $\zeta$ with itself $N$ times. Unfortunately, for an old sympletic manifold, 
$\zeta \wedge \zeta=0$. Hence, a measure must be introduced by hand:

$$
d \mu(z)=\rho(z) \prod_{a=1}^{2 N} d z^{a}
$$

where $\rho(z)$ is a density. The divergence of a generic vector field $\overleftarrow{V}=\frac{\overleftarrow{\partial}}{\partial z^{a}} V^{a}$ is defined in the usual way by

$$
\operatorname{div}_{\rho} \overleftarrow{V} \equiv \frac{1}{\rho}(-1)^{\epsilon_{a}} \frac{\partial_{l}\left(\rho V^{a}\right)}{\partial z^{a}}
$$

Then, the laplacian $\Delta_{\rho}$ acting on a function $X$ is defined by taking the divergence of the corresponding vector field via

$$
\Delta_{\rho} X \equiv(-1)^{\epsilon_{X}} \frac{1}{2} \operatorname{div}_{\rho} \overleftarrow{V}_{X}=(-1)^{\epsilon_{X}+\epsilon_{a}} \frac{1}{2 \rho} \frac{\partial_{l}}{\partial z^{a}}\left(\rho \zeta^{a b} \frac{\partial_{l} X}{\partial z^{b}}\right) .
$$

Since one would like to use $\Delta_{\rho}$ as the general coordinate version of $\Delta$ of Sect. 6.1, one wants it to be nilpotent. However, this is not necessarily the case since

$$
\Delta_{\rho} \Delta_{\rho}=\frac{1}{2}\left[\Delta_{\rho}\left(\frac{1}{\rho}(-1)^{\epsilon_{a}+\epsilon_{b}} \frac{\partial_{l}}{\partial z^{a}}\left(\rho \zeta^{a b}\right)\right)\right] \frac{\partial_{l}}{\partial z^{b}} .
$$

Hence, one requires $\rho$ to satisfy

$$
\Delta_{\rho}\left(\frac{1}{\rho}(-1)^{\epsilon_{a}+\epsilon_{b}} \frac{\partial_{l}}{\partial z^{a}}\left(\rho \zeta^{a b}\right)\right)=0 .
$$

When Eq. (10.48) holds, $\Delta_{\rho}$ is formally nilpotent and a graded derivation of both functional multiplication and the antibracket, i.e., it satisfies Eq.(6.8) with $\Delta \rightarrow \Delta_{\rho}$.

It turns out that Eq.(10.48) is the necessary and sufficient condition for the existence of Darboux coordinates locally. For such coordinates, $\rho=1$ and $\zeta^{a b}$ takes the form in Eq.(4.8). Then $z^{a}$ for $a=1, \ldots, N$ can be identified with fields and $z^{a}$ for $a=N+1, \ldots, 2 N$ can be identified with antifields. Hence, we employed the Darboux coordinate system for the antibracket formalism in Sects. 1 -8. Darboux coordinates suffice as long as global issues are not important.

Quantization in a general coordinate system proceeds as in the Darboux case. Everywhere $\Delta$ appears in Sects. 6- 6 , one replaces it by $\Delta_{\rho}$. The functional-integral measure also must be modified. Integration is restricted to an $N$-dimensional submanifold $\mathcal{N}$. Since little distinction is made between fermionic and bosonic coordinates in the covariant formulation, $\mathcal{N}$ can be an arbitrary $(k, N-k)$ submanifold as long as $\zeta\left[V, V^{\prime}\right]=0$ on $\mathcal{N}$ for any two tangent vectors $V, V^{\prime} \in \mathcal{T} \mathcal{N}$. One considers a basis $\left\{e_{1}, \ldots, e_{N} ; h^{1}, \ldots, h^{N}\right\}$ for $\mathcal{T} \mathcal{M}$, such that $\left\{e_{1}, \ldots, e_{N}\right\}$ is a basis for $\mathcal{T} \mathcal{N}$ and $\zeta\left[e_{i}, h^{j}\right]=\delta_{i}^{j}$. Then the volume element on $\mathcal{N}$ is

$$
d \mu_{\mathcal{N}}\left(e_{1}, \ldots, e_{N}\right)=\left[d \mu\left(e_{1}, \ldots, e_{N} ; h^{1}, \ldots, h^{N}\right)\right]^{1 / 2} .
$$


Since integration in function space is restricted to $\mathcal{N}$, the above procedure corresponds to a gauge-fixing procedure. The submanifold $\mathcal{N}$ can be defined using $N$ linearly independent constraints $\Psi_{A}(z)=0$ satisfying

$$
\left(\Psi_{A}, \Psi_{B}\right)=T_{A B}^{C}(z) \Psi_{C}
$$

The vectors $\overleftarrow{V}_{\Psi_{A}}$ are a basis for the tangent space $\mathcal{T} \mathcal{N}$ of $\mathcal{N}$. Furthermore, as a consequence of Eq. (10.50), $\zeta\left[\overleftarrow{V}_{\Psi_{A}}, \overleftarrow{V}_{\Psi_{B}}\right]=0$ on $\mathcal{N}$, which is a consistency check. To make contact with the gauge-fixing procedure of Sect. 6, one goes to the Darboux coordinate system and chooses $\Psi_{A}=\Phi_{A}^{*}-\frac{\partial \Psi}{\partial \Phi^{A}}$. One disadvantage of the general coordinate approach, is that the concept of ghost number becomes obscure.

\subsection{The $\mathrm{D}=26$ Closed Bosonic String Field Theory}

A review of the current formulation of the closed bosonic string has been given in ref.[281]. Here, we present some of the salient points.

At the first-quantized level, closed strings possess holomorphic factorization. This means that, with the possible exception of zero modes, the integrands of closed-string amplitudes factorize into two open-string-like integrands, one for left-moving degrees of freedom and one for right-moving degrees of freedom. At the second-quantized level, there is a similar splitting. Hence, a closed string field $A$ is a tensor product of a left string field $A_{L}$ with a right string field $A_{R}$, so that one can write $A$ as

$$
A=\left(A_{L} ; A_{R}\right) \text {, }
$$

or as a sum of terms of the form in Eq.(10.51). The field $A_{L}$ (respectively, $A_{R}$ ) is precisely of the form of the open string case, except a subscript $L$ (respectively, $R$ ) is appended to all quantities. One exception is the zero modes of $X^{\mu}(\sigma)$, namely the position and momentum operators. They are the same for both left and right sectors, so that $x_{L}^{\mu}=x_{R}^{\mu} \equiv x^{\mu}$ and $p_{L}^{\mu}=p_{R}^{\mu} \equiv p^{\mu}$. The total string ghost number is the sum of the left and right string ghost numbers, i.e., $g(A)=g_{L}\left(A_{L}\right)+g_{R}\left(A_{R}\right)$.

One can attempt to construct closed-string field theory along the lines of the open string case described in Sect. 3.6. It is easy to see that not all the open string axioms can be extended. When the axioms hold, Paton-Chan factors [208] can be appended to the string field leading to a non-abelian Yang-Mills gauge group. However, closed string theories cannot possess such a non-abelian gauge structure [220].

Define closed-string integration $\int_{\text {closed }}$ as the product of left and right open string integrals via $\int_{\text {closed }} \equiv \int_{L} \int_{R}$, i.e., for fields in the form in Eq.(10.51), one has

$$
\int_{\text {closed }} A=\int_{L} A_{L} \int_{R} A_{R}
$$


To generate a non-zero integral in Eq.(10.52), $A$ must have a left-ghost number of 3 and a right-ghost number of 3 and consequently a total ghost number of 6 :

$$
\int_{\text {closed }} A=0, \quad \text { if } g(A) \neq 6 \text {. }
$$

Let $\sigma$ be the first-quantized variable parametrizing the string. It varies between 0 and $2 \pi$ and is periodic. To define the closed-string star operation $\circ$, pick two antipodal points, e.g. $\sigma=0$ and $\sigma=\pi$. This divides a string into two halves. Then, $\circ$ is defined in analogy to the open string case. One half of one string overlaps with one half of the other string and what remains is the product string. The first-quantized BRST charge $Q$ is the sum of the right and left parts:

$$
Q=Q_{L}+Q_{R}
$$

and it carries ghost number one: $g(Q)=1$. Eq.(10.53) is equivalent to

$$
Q A=\left(Q_{L} A_{L} ; A_{R}\right)+\left(A_{L} ; Q_{R} A_{R}\right) \quad .
$$

Even though $\int, \circ$ and $Q$ have been defined, there is a difficulty in obtaining a free action. Let $C$ denote the closed-string field. The naive term $\int C \circ Q C$ vanishes because of ghost number considerations. The total ghost number of the integrand, which is $2 g(C)+1$, must be equal to 6 . This constraint cannot be satisfied because $g(C)$ is an integer.

To correct the problem, various schemes can be used [9, 192, 168]. Let

$$
\bar{c}_{0}^{-} \equiv \bar{c}_{0}^{L}-\bar{c}_{0}^{R}, \quad c_{0}^{-} \equiv \frac{1}{2}\left(c_{0}^{L}-c_{0}^{R}\right) .
$$

Impose the following two constraints on $C$

$$
\bar{c}_{0}^{-} C=0, \quad\left(L_{0}^{L}-L_{0}^{R}\right) C=0 .
$$

Anticipating that the free-theory equation is $Q C=0$, one sees that these constraints are consistent since $\left\{Q, \bar{c}_{0}^{-}\right\}=L_{0}^{L}-L_{0}^{R},\left[Q, L_{0}^{L}-L_{0}^{R}\right]=0$ and $\left[\bar{c}_{0}^{-}, L_{0}^{L}-L_{0}^{R}\right]=0$. The condition $\left(L_{0}^{L}-L_{0}^{R}\right) C=0$ is quite natural. The operator $L_{0}^{L}-L_{0}^{R}$ is the generator for rigid rotation of the first-quantized parameter $\sigma$, which labels the points along the string. Since, in the closed-string case, $\sigma$ is periodic, there is no preferred choice of an origin. The condition $\left(L_{0}^{L}-L_{0}^{R}\right) C=0$ does not lead to an equation of motion for $C$ since the $\frac{1}{2} \partial_{\mu} \partial^{\mu}$ terms in $L_{0}^{L}$ and $L_{0}^{R}$ cancel. From Eq.(7.34), one sees that it implies that the mass $\mathcal{M}_{L}$ of the left-sector must equal the mass $\mathcal{M}_{R}$ of the right-sector, a well-known constraint of first-quantized closed-string states. However, 
the operation $\circ$ does not preserve the constraint. One can modify $\circ$ to ô by averaging over a rigid rotation that rotates the product string over angles ranging from 0 to $2 \pi$. The new ô no longer is associative, as can be checked by drawing some pictures.

The ghost number problem can be fixed by inserting a factor of $c_{0}^{-}$. Define the quadratic form

$$
\langle A, B\rangle=\int_{\text {closed }} A \hat{o} c_{0}^{-} B
$$

Let the free action be

$$
S_{0}^{(2)}=\frac{1}{2}\langle C, Q C\rangle
$$

It is invariant under the gauge transformations $\delta C=Q \Lambda$, where $g(\Lambda)=1$ since $g(C)=2$. There are many ways of resolving the difficulty with ghost number of the free action of closed-string field theory but they are equivalent to the above. The tree-level three-point interaction is

$$
S_{0}^{(3)}=\frac{2 g}{3} \int_{\text {closed }} C \hat{o} C \hat{o} C
$$

For on-shell external states, this interaction correctly produces three-point interactions.

Tree-level gauge invariance is violated for the theory described by $S_{0}^{(2)}+S_{0}^{(3)}$ due to the violation of the associativity axiom. However, by adding higher-order terms gauge invariance can be restored [168, 169, 170, 180, 212, 183, 280]. The new interactions can be defined by relatively simple geometrical constraints 280, 281. This leads to a tree-level non-polynomial closed-string field theory. Unfortunately, the classical theory needs further modification at the quantum level. One-loop and higher-loop amplitudes are not produced by using only tree-level vertices. It is at this stage where the antibracket formalism has been of great utility. Interaction terms proportional to powers of $\hbar$ need to be added in a manner similar to Eq.(6.14). To ensure that the theory is quantum-mechanically gauge invariant, the work in refs. 280, 224, 281, 282, 149, 225 has relied on the antibracket formalism. The guiding principle is that the quantum closed-string field theory must satisfy the quantum master equation.

The antibracket is defined using the quadratic form in Eq.(10.57) [224, 281, 282. As in the open string field theory, the system is infinitely reducible so that there are ghosts for ghosts ad infinitum. The fields can be collected into one object $\Psi_{c}$ in a manner similar to the open string case in Eq. (5.23)

$$
\Psi_{c} \equiv \ldots+{ }^{*}{ }^{*} \mathcal{C}_{s}^{*}+\ldots+{ }^{*} \mathcal{C}_{0}^{*}+{ }^{*} C^{*}+\stackrel{2}{C}+\stackrel{1}{\mathcal{C}_{0}}+\ldots+{ }^{-s} \mathcal{C}_{s}+\ldots
$$


where the ghost number is indicated above the field. The close-string Hodge operation, denoted by a superscript $*$ in front of a field, is defined using the quadratic form in Eq.(10.57). It takes a $p$-form into a $5-p$, where the order of string form is the same as the ghost number.

Let $\varphi_{s}$ be a complete set of normalized first-quantized states for $g\left(\varphi_{s}\right) \leq 2$. Let ${ }^{*} \varphi^{s}$ denote the corresponding state transformed by the Hodge star operation. The ${ }^{*} \varphi^{s}$ are a normalized complete set of states for ghost numbers greater than 2. With these definitions, $\Psi_{c}$ in Eq.(10.60) can be written in terms of ordinary particle fields $\psi^{s}$ via $\Psi_{c}=\sum_{s}\left(\varphi_{s} \psi^{s}+{ }^{*} \varphi^{s} \psi_{s}^{*}\right)$ where $\psi_{s}^{*}$ are the antifields of $\psi^{s}$. The quantum master equation is then the same as the particle case, namely $\frac{1}{2}\left(W\left(\Psi_{c}\right), W\left(\Psi_{c}\right)\right)=$ $i \hbar \Delta W\left(\Psi_{c}\right)$, where the antibracket is $\left(X\left(\Psi_{c}\right), Y\left(\Psi_{c}\right)\right)=\frac{\partial_{r} X}{\partial \psi^{s}} \frac{\partial_{l} Y}{\partial \psi_{s}^{*}}-\frac{\partial_{r} X}{\partial \psi_{s}^{*}} \frac{\partial_{l} Y}{\partial \psi^{s}}$ and $\Delta=$ $(-1)^{\epsilon_{s}+1} \frac{\partial_{r}}{\partial \psi^{s}} \frac{\partial_{r}}{\partial \psi_{s}^{*}}$.

The solution of the quantum master equation for the closed-string field theory is presented in refs. [281, 282]. This tour-de-force work goes beyond the goals of our review. The reader interested in this topic can consult the above references for more discussion.

The current formulation of string fields theories is developed around a particular space-time background. Any background is permitted, as long as it leads to a nilpotent first-quantized BRST charge. Such BRST charges correspond to two-dimensional conformal field theories with the total central charge of the Virasoro algebra equal to zero. Usually, the flat space-time background in 26 dimensions is used. Since string theories contain gravity, it should be possible to pass from one background to another. It is an interesting question of whether there is background independence of string field theory [223, 222, 274, 224, 275, 225]. A proof for bosonic string field theories has been obtained in refs. 2224, 225], for backgrounds infinitesimally close. The antibracket formalism has played an important role in the analysis. The basic idea is that string field theory, formulated about a particular background $\mathcal{B}$, corresponds to a particular solution $S_{\mathcal{B}}$ of the classical master equation. Reference [224] demonstrated that, for two nearby backgrounds $\mathcal{B}_{1}$ and $\mathcal{B}_{2}, S_{\mathcal{B}_{1}}$ and $S_{\mathcal{B}_{2}}$ are related by a canonical transformation of the antibracket. The conclusion is that string field theory is background independent, although not manifestly. Barring difficulties with singular expressions, ref. 2225 has extended the result to the quantum case. For the quantum system, a particular background $\mathcal{B}$ corresponds to a solution $W_{\mathcal{B}}$ of the quantum master equation. 


\subsection{Extended Antibracket Formalism for Anomalous Gauge Theories}

In certain cases, it is possible to quantize an anomalous gauge theory. An example is the first-quantized bosonic string for $D \neq 26$ discussed in Sect. 9.3. Polyakov [210] quantized this system in the presence of a conformal anomaly. A new degree of freedom, the Liouville mode, emerged. Another example is the chiral Schwinger model in two dimensions. Despite its anomalous behavior, it is consistent and unitary [166]. In refs. [97, 99], additional degrees of freedom were introduced into four-dimensional anomalous Yang-Mills theories to cancel the anomalies in the path integral. This cancellation can be obtained by adding a Wess-Zumino term for the extra degrees of freedom. A careful treatment of the integration measure in Faddeev-Popov pathintegral quantization shows how such a Wess-Zumino term can arise naturally [207]. For earlier approaches to this subject in the case of the Schwinger model, see [7, 145]. A treatment of anomalous chiral QCD in two dimensions within the field-antifield formalism was obtained in refs. 61, 63]. Methods of quantizing anomalous gauge theories using the antibracket formalism were developed in [61, 62, 131, 133, 134, 77, 63.

Let us describe in general terms the extended antibracket method of refs. 131, 133, 134. For simplicity we consider the closed irreducible case. At the classical level, the number of dynamical local degrees of freedom $n_{\text {dof }}$ is the total number of fields $n$ minus the number of gauge invariances $m_{0}$, i.e., $n_{\text {dof }}=n-m_{0}$. Suppose there are $r$ anomalous gauge invariances. Then, due to quantum effects, $r$ of the $m_{0}$ gauge degrees of freedom enter the theory dynamically. Hence, the true net number of degrees of freedom at quantum level is $n-m_{0}+r$. Following the ideas of refs. 268, 210, 279, 97, 99] for treating anomalous gauge theories, one wants to have $r$ extra degrees of freedom. The proposal is to augment the original set of fields, $\phi^{i}$, $i=1,2, \ldots, n$ with $r$ new fields $\widehat{\phi}^{\hat{i}}, \hat{i}=1,2, \ldots, r$, in such a way that the original gauge structure continues to be maintained at classical level. Roughly speaking, the $\widehat{\phi}$ are fields parametrizing the anomalous part of the gauge group. In what follows, a "hat" on a quantity indicates that the quantity is associated with the extra degrees of freedom or that the quantity has been generalized to the extended system.

The key step is to extend the antibracket formalism to include $\widehat{\phi}$ variables. To implement this idea, one demands the extra fields to transform under the action of the gauge group:

$$
\delta \widehat{\phi}^{\hat{i}}=\widehat{R}_{\alpha}^{\hat{i}}[\phi, \widehat{\phi}] \varepsilon^{\alpha} .
$$

Let $\widehat{\phi}_{\hat{i}}^{*}$ be the antifield of $\widehat{\phi}^{\hat{i}}$. Given Eq. (4.29), the classical gauge structure of the extended theory should be governed by an action $\widetilde{S}=S+\widehat{\phi}_{\hat{i}}^{*} \widehat{R}_{\alpha}^{\hat{i}} \mathcal{C}^{\alpha}$. Since field indices 
range over $a=1,2, \ldots, n, n+1, \ldots, n+r$, the compact notation $\phi^{a} \equiv\left[\phi^{i}, \widehat{\phi}^{\hat{\imath}}\right]$ and $R_{\alpha}^{a} \equiv\left[R_{\alpha}^{i}, \hat{R}_{\alpha}^{\hat{i}}\right]$ is useful. To maintain the gauge structure, the extended generators $R_{\alpha}^{a}$ must satisfy the original gauge algebra. In other words, when the field content is extended, Eq.(2.22) must still hold, where the tensors $T$ and $E$ are functions of the $\phi^{i}$ only and have the same values as in the unextended theory. This requirement leads to a set of equations for the generators $\widehat{R}_{\alpha}^{\hat{i}}$ in Eq.(10.61). Formulas for $\widehat{R}_{\alpha}^{\hat{i}}$ in the closed case for which $E=0$ are given in refs. [131, 133]. In the closed case, it is possible to solve the antifield independent part of the original quantum master equation at one-loop in a quasilocal way in the extended theory. In particular, the Wess-Zumino term $M_{1}[\phi, \widehat{\phi}]$ can be written as

$$
M_{1}[\phi, \widehat{\phi}]=-i \int_{0}^{1} d s \mathcal{A}_{\hat{i}}(F[\phi, \widehat{\phi} s]) \hat{\phi}^{\hat{i}}
$$

where the $\mathcal{A}_{\hat{i}}$ are the antifield-independent part of the anomalies and $F^{i}$ is the finite gauge transformation of the classical fields $\phi^{i}$ under the anomalous part of the group. The BRST variation of $M_{1}$ gives the original anomaly: $\left(M_{1}, \widetilde{S}\right)=i \mathcal{A}_{\alpha} \mathcal{C}^{\alpha}$.

However, in the extended antibracket formalism the action $\widetilde{S}$ is not proper [77]. To have a well defined perturbation expansion, it is necessary to modify $\widetilde{S}$ to a new extended action $\widehat{S}$ that satisfies the classical master equation

$$
(\widehat{S}, \widehat{S})=0
$$

and is proper, i.e.,

$$
\left.\operatorname{rank} \frac{\partial_{l} \partial_{r} \widehat{S}}{\partial z^{a} \partial z^{b}}\right|_{\text {on-shell }}=n+m_{0}+r
$$

where the $z^{a}$ include $n$ fields, $r$ extended fields, $m_{0}$ ghosts, and their antifields. One proposal for $\widehat{S}$ would be $\widetilde{S}+\hbar M_{1}$. A difficulty is the presence of an order $\hbar$ term. However, in certain cases a canonical transformation which scales $\widehat{\phi}^{\hat{i}}$ by $1 / \hbar^{\frac{1}{2}}$ [77] can be performed to overcome the problem. The general structure for $\widehat{S}$ for anomalous gauge theories with an anomalous abelian subgroup is

$$
\widehat{S}=S-\frac{i}{2} \widehat{\phi}^{\hat{i}} \widehat{D}_{\hat{i} \hat{j}} \widehat{\phi}^{\hat{j}}+\widehat{\phi}_{\hat{i}}^{*} \widehat{T}_{\beta \hat{j}}^{\hat{i}} \widehat{\phi}^{\hat{j}} \mathcal{C}^{\beta}
$$

where $\widehat{D}$ and $\widehat{T}$ are tensors which can be found in ref. 133. In particular, $\widehat{D}_{\hat{i} \hat{j}}$ is related to the BRST variation of the anomalies $\mathcal{A}_{\hat{i}}$. Since $\widehat{S}$ satisfies the master equation, a classical BRST symmetry can be defined using $\delta_{B} X=(X, \widehat{S})$.

The final stage is to find a solution $\widehat{W}$ to the quantum master equation in the extended space. Because of the above-mentioned scaling of $\widehat{\phi}, \widehat{W}$ is a series in $\hbar^{\frac{1}{2}}$ [77, 257] rather than $\hbar: \widehat{W}=\widehat{S}+\hbar^{\frac{1}{2}} \widehat{M}_{\frac{1}{2}}+\ldots$. After regulating the extended theory, $(\Delta \widehat{S})_{\text {reg }}$ 
is computed. By appropriately adjusting $\widehat{S}$ and $\widehat{M}_{\frac{1}{2}}$, the antifield-independent part of the complete one-loop obstruction $\widehat{\mathcal{A}}_{1}$ to the quantum master equation in the extended space can be cancelled. In terms of the unscaled extra variables, this adjustment corresponds to a finite renormalization of the original expression of the Wess-Zumino term in Eq. (10.62), $M_{1} \rightarrow \widehat{M}_{1}$. Note that the locality of $\widehat{M}_{\frac{1}{2}}$ cannot be guaranteed. Likewise, locality of the renormalized Wess-Zumino term is not assured due to the integral over the variable $s$ in expressions like (10.62). In some cases, such as the first-quantized bosonic string [131, 133] or the abelian Schwinger model [207], only local action terms are generated. Then, the anomalous theory makes sense at the quantum level. However, for chiral QCD in two dimensions [131], the integral over $s$ remains. Even in these cases, the violation of locality is in some sense not severe: The equations of motion are local, a situation referred to as quasilocal. When the quantum extended theory is well-defined, the final stage, namely gauge-fixing, proceeds in a manner similar to the non-anomalous case [131].

\section{Acknowledgements}

We thank G. Barnich, C. Batlle, F. De Jonghe, M. Henneaux, A. K. Kostelecký, J. M. Pons, J. Roca, R. Siebelink, A. Slavnov, P. Townsend, W. Troost, S. Vandoren, A. Van Proeyen and F. Zamora for discussions. This work is supported in part by the Comisión Interministerial para la Ciencia y la Technología (project number AEN-0695), by a Human Capital and Mobility Grant (ERB4050PL930544), by the National Science Foundation (grant number PHY-9009850), by a NATO Collaborative Research Grant (0763/87), by the Robert A. Welch Foundation, and by the United States Department of Energy (grant number DE-FG02-92ER40698). 


\section{A Appendix: Right and Left Derivatives}

In this appendix, we provide more details about left and right derivatives [38, 40, [188, 85, 39]. For any function or functional $X$ of $\phi$, they are defined as

$$
\frac{\partial_{l} X}{\partial \phi} \equiv \frac{\vec{\partial}}{\partial \phi} X, \quad \frac{\partial_{r} X}{\partial \phi} \equiv X \frac{\overleftarrow{\partial}}{\partial \phi}
$$

Left derivatives are the ones usually used. Right derivatives act from right to left. The differential $d X(\phi)$ of $X$ is

$$
d X(\phi)=d \phi \frac{\partial_{l} X}{\partial \phi}=\frac{\partial_{r} X}{\partial \phi} d \phi
$$

The formula for the variation $\delta X$ of $X$ with respect to $\phi$ resembles Eq.(A.2):

$$
\delta X(\phi)=\delta \phi \frac{\partial_{l} X}{\partial \phi}=\frac{\partial_{r} X}{\partial \phi} \delta \phi
$$

What is the relation between left and right derivatives? If $\phi$ is commuting $(\epsilon(\phi)=$ $0)$, then $\frac{\partial_{l} X}{\partial \phi}=\frac{\partial_{r} X}{\partial \phi}$, so that one only needs to be careful when $\phi$ is anticommuting $(\epsilon(\phi)=1)$. Assume $\phi$ is anticommuting. Then $\phi \phi=0$. Without loss of generality we may assume that $X=\phi Y+Z$ where $Y$ and $Z$ have no $\phi$ dependence. The left and right derivatives of $X$ are then $\frac{\partial_{l} X}{\partial \phi}=Y$ and $\frac{\partial_{r} X}{\partial \phi}=(-1)^{\epsilon_{Y}} Y=(-1)^{\epsilon(\phi)\left(\epsilon_{X}+1\right)} Y$. For all cases,

$$
\frac{\partial_{l} X}{\partial \phi}=(-1)^{\epsilon(\phi)\left(\epsilon_{X}+1\right)} \frac{\partial_{r} X}{\partial \phi}
$$

As a pedagogical exercise, let us derive the graded antisymmetry property of the bracket in Eq.(4.5). Start with the definition of $(Y, X)$ in Eq.(4.4) and interchange the order of derivatives to obtain

$$
\begin{gathered}
(Y, X)=\frac{\partial_{r} Y}{\partial \Phi^{A}} \frac{\partial_{l} X}{\partial \Phi_{A}^{*}}-\frac{\partial_{r} Y}{\partial \Phi_{A}^{*}} \frac{\partial_{l} X}{\partial \Phi^{A}}= \\
(-1)^{\left(\epsilon_{Y}+\epsilon_{A}\right)\left(\epsilon_{X}+\epsilon_{A}+1\right)} \frac{\partial_{l} X}{\partial \Phi_{A}^{*}} \frac{\partial_{r} Y}{\partial \Phi^{A}}-(-1)^{\left(\epsilon_{X}+\epsilon_{A}\right)\left(\epsilon_{Y}+\epsilon_{A}+1\right)} \frac{\partial_{l} X}{\partial \Phi^{A}} \frac{\partial_{r} Y}{\partial \Phi_{A}^{*}} .
\end{gathered}
$$

Then using Eq.(A.4), one finds that the above is equal to

$$
\begin{gathered}
(-1)^{\epsilon_{X} \epsilon_{Y}+\epsilon_{X}+\epsilon_{Y}+1} \frac{\partial_{r} X}{\partial \Phi_{A}^{*}} \frac{\partial_{l} Y}{\partial \Phi^{A}}-(-1)^{\epsilon_{X} \epsilon_{Y}+\epsilon_{X}+\epsilon_{Y}+1} \frac{\partial_{r} X}{\partial \Phi^{A}} \frac{\partial_{l} Y}{\partial \Phi_{A}^{*}} \\
=-(-1)^{\left(\epsilon_{X}+1\right)\left(\epsilon_{Y}+1\right)}(X, Y) .
\end{gathered}
$$

This is the desired result. 
As another exercise, let us verify the formulas for $(F, F)$ and $(B, B)$ in Eq.(4.6) When $Y=X$, the second term in Eq.4.4) can be written as

$$
\frac{\partial_{r} X}{\partial \Phi_{A}^{*}} \frac{\partial_{l} X}{\partial \Phi^{A}}=(-1)^{\left(\epsilon_{X}+1\right)\left(\epsilon_{X}+1\right)} \frac{\partial_{r} X}{\partial \Phi^{A}} \frac{\partial_{l} X}{\partial \Phi_{A}^{*}},
$$

using the same manipulations as in the previous paragraph. When $X=F$ is anticommuting, the sign factor is plus and the second term in Eq.(4.4) cancels the first. When $X=B$ is commuting, the sign factor is minus and the two add.

Another useful result concerns integration by parts. When $\phi$ is commuting, one has the standard formula $\int \mathrm{d} \phi \frac{\partial_{r} X}{\partial \phi} Y=-\int \mathrm{d} \phi X \frac{\partial_{l} Y}{\partial \phi}$. In such a case, $\frac{\partial_{r}}{\partial \phi}=\frac{\partial_{l}}{\partial \phi}$, so that the left and right subscripts on $\partial$ are inconsequential. Suppose $\epsilon(\phi)=1$. Then, $\int \mathrm{d} \phi \frac{\partial_{r} X}{\partial \phi} Y$ and $\int \mathrm{d} \phi X \frac{\partial_{l} Y}{\partial \phi}$ are both zero unless both $X$ and $Y$ are linear in $\phi$. Without loss of generality, we may assume that $X=x \phi$ and $Y=\phi y$ where $x$ and $y$ are independent of $\phi$. Then, $\int \mathrm{d} \phi \frac{\partial_{r} X}{\partial \phi} Y=\int \mathrm{d} \phi x(\phi y)=(-1)^{\epsilon(x)} x y$ and $\int \mathrm{d} \phi X \frac{\partial_{l} Y}{\partial \phi}=$ $\int \mathrm{d} \phi(x \phi) y=(-1)^{\epsilon(x)} x y$ lead to the same result. Summarizing, all cases are contained in the formula

$$
\int \mathrm{d} \phi \frac{\partial_{r} X}{\partial \phi} Y=(-1)^{\epsilon(\phi)+1} \int \mathrm{d} \phi X \frac{\partial_{l} Y}{\partial \phi}
$$

For second derivatives of $X$, one has

$$
\begin{gathered}
\frac{\partial_{r} \partial_{l} X}{\partial \phi \partial \phi^{\prime}}=\frac{\partial_{l} \partial_{r} X}{\partial \phi^{\prime} \partial \phi}, \\
\frac{\partial_{l} \partial_{l} X}{\partial \phi \partial \phi^{\prime}}=(-1)^{\epsilon(\phi) \epsilon\left(\phi^{\prime}\right)} \frac{\partial_{l} \partial_{l} X}{\partial \phi^{\prime} \partial \phi} \\
\frac{\partial_{r} \partial_{r} X}{\partial \phi \partial \phi^{\prime}}=(-1)^{\epsilon(\phi) \epsilon\left(\phi^{\prime}\right)} \frac{\partial_{r} \partial_{r} X}{\partial \phi^{\prime} \partial \phi}
\end{gathered}
$$

In the first equation, derivatives act from different directions and hence commute. In the second and third equations, one must be careful of the order.

If $X$ is a functional of $Y$ which is a function of $\phi$, one has the chain rules

$$
\begin{gathered}
\frac{\partial_{r} X(Y(\phi))}{\partial \phi}=\frac{\partial_{r} X}{\partial Y} \frac{\partial_{r} Y}{\partial \phi} \\
\frac{\partial_{l} X(Y(\phi))}{\partial \phi}=\frac{\partial_{l} Y}{\partial \phi} \frac{\partial_{l} X}{\partial Y} .
\end{gathered}
$$




\section{B Appendix: The Regularity Condition}

If a theory is invariant under the gauge transformations in Eq. (2.1) then the lagrangian does not depend on all degrees of freedom. In other words, $S_{0}\left[\phi^{\prime}\right]=S_{0}[\phi]$ where $\phi^{\prime}$ stands for finitely transformed fields produced by any of the infinitesimal variations in Eq.(2.1). When this relation is expanded to first order in the gauge parameters $\varepsilon^{\alpha}$, the Noether relations in Eq.(2.8) are obtained.

Let $\phi_{0}$ be the stationary point about which one would like to perform the perturbative expansion. Then, in a neighborhood of $\phi_{0}$ there are other stationary points given by performing finite gauge transformations on $\phi_{0}$. Let $\Sigma$ locally be the surface around $\phi_{0}$ in $\phi$ configuration space where the equations of motion vanish. The regularity condition assumes that the dimension of $\Sigma$ is maximal and that the quadratic form generated by expanding the lagrangian to second order in fields has a rank $n_{\text {dof }}$ on this surface 28]. Hence, the number of fields that enter dynamically in $S_{0}$ is $n_{\text {dof }}$. The regularity assumption is important for implementing perturbation theory since the propagator - which is the inverse of this quadratic form - then exists.

Summarizing, the regularity condition is

$$
\left.\operatorname{rank} \frac{\partial_{l} S_{0, i}}{\partial \phi^{j}}\right|_{\Sigma}=\left.\frac{\partial_{l} \partial_{r} S_{0}}{\partial \phi^{i} \partial \phi^{j}}\right|_{\Sigma}=n_{\text {dof }}
$$

where $\Sigma$ is the stationary surface defined implicitly by

$$
\left.S_{0, i}\right|_{\Sigma}=0
$$

In other words, the on-shell degeneracy of the hessian in Eq.(B.1) is completely due to the $n-n_{\text {dof }}$ independent null vectors $R_{\alpha}^{i}$ associated with gauge transformations and does not come from some other source 27, 28]:

$$
\left.\frac{\partial_{l} \partial_{r} S_{0}}{\partial \phi^{i} \partial \phi^{j}} R_{\alpha}^{i}\right|_{\Sigma}=0
$$

An example of a lagrangian that does not satisfy the regularity condition is $\mathcal{L}=\phi^{4}$ with no kinetic energy term for $\phi$. The stationary point $\phi_{0}=0$ has a vanishing quadratic form even though there is no gauge invariance. In such a case one can proceed by arbitrarily adding and subtracting some kinetic energy term and treating $\phi^{4}$ minus this kinetic energy term as a perturbation. However, throughout this review we assume that such singular cases do not arise.

In principle, one can separate the degrees of freedom into propagating degrees of freedom $\varphi^{s}, s=1,2, \ldots, n_{\text {dof }}$ and gauge degrees of freedom $\chi^{a}, a=1,2, \ldots, n-$ $n_{\text {dof }}$. An efficient separation is often difficult and the $\varphi^{s}$ and $\chi^{a}$ are usually quite complicated and nonlocal functionals of the $\phi^{i}$. 
The regularity conditions are then given by

$$
\begin{aligned}
& S_{0, a}=0, \quad \text { identically }, \\
& \left.\operatorname{rank} \frac{\partial_{l} S_{0, s}}{\partial \phi^{i}}\right|_{\phi=\phi_{0}}=n_{\text {dof }} .
\end{aligned}
$$

The regularity condition assumes that the equations of motion $S_{0, i}$ constitute a regular representation of the stationary surface $\Sigma$. This means that the functions $S_{0, i}$ can be locally split into independent, $G_{s}$, and dependent ones, $G_{a}$, in such a way that

1. $G_{a}=0$ are a direct consequence of $G_{s}=0$, and

2. The rank of the matrix of the gradients $d G_{s}$ is maximal on $\Sigma$.

In other words, the regularity condition ensures that locally the changes of variables $\phi^{i} \rightarrow\left[\varphi^{s}, \chi^{a}\right]$ or $\phi^{i} \rightarrow\left[G_{s}, G_{a}\right]$ makes sense [27, 28, 105].

When the regularity condition is fulfilled, it can be shown that any smooth function that vanishes on the stationary surface $\Sigma$ can be written as a combination of the equations of motion [28, 103, 105, 106], i.e.,

$$
\left.F(\phi)\right|_{\Sigma}=0 \Rightarrow F(\phi)=\lambda^{j} S_{0, j} \quad,
$$

where the $\lambda^{j}$ may be functions of the $\phi^{i}$. No restrictions are made on the $\lambda^{j}(\phi)$. Putting restrictions can lead to violations of (B.5). An example is presented in [257]. By considering only local functionals, ref. [257] found cases for which Eq. (B.5) could not be satisfied as a local combination of the equations of motion.

For more details on regularity conditions as well as derivations of the above results consult references [81, 27, 28, 106, 103, 105, 152. 


\section{Appendix: Anomaly Trace Computations}

In this appendix, we perform the functional trace calculations of Sect. 9. One key idea is to use the Dyson-like expansion 102, 165

$$
\begin{array}{r}
\exp \left[\mathcal{R}_{0}+V\right]=\exp \left[\mathcal{R}_{0}\right]+\int_{0}^{1} \mathrm{~d} s \exp \left[(1-s) \mathcal{R}_{0}\right] V \exp \left[s \mathcal{R}_{0}\right]+ \\
\int_{0}^{1} \mathrm{~d} u \int_{0}^{u} \mathrm{~d} s \exp \left[(1-u) \mathcal{R}_{0}\right] V \exp \left[(u-s) \mathcal{R}_{0}\right] V \exp \left[s \mathcal{R}_{0}\right]+\ldots
\end{array}
$$

Typically, $\mathcal{R}_{0}$ is independent of the cutoff $\mathcal{M}$, and $V$ goes like inverse powers of $\mathcal{M}$ so that only a few terms in Eq. (C.1) need to be kept.

The anomaly equation Eq. 8.53) involves a functional trace [120. If one uses momentum-space eigenfunctions to saturate the sum, then expressions such as

$$
\exp (-i k \cdot x)\left(O\left(\partial_{\mu}\right)\right) \exp (i k \cdot x)
$$

arise where $O\left(\partial_{\mu}\right)$ is an arbitrary operator, or a product of operators, involving the derivative $\partial_{\mu}$. By commuting $\exp (i k \cdot x)$ through the expression, one arrives at

$$
\exp (-i k \cdot x)\left(O\left(\partial_{\mu}\right)\right) \exp (i k \cdot x)=\left(O\left(\partial_{\mu}+i k_{\mu}\right)\right) 1
$$

When derivatives in $O\left(\partial_{\mu}+i k_{\mu}\right)$ act on the function 1 , they produce zero.

For the spinless relativistic particle system, we begin by taking Eq.(9.18) and commuting $\exp (i k \tau)$ through the expression, using Eq.(C.2), to obtain

$$
\begin{gathered}
(\Delta S)_{\text {reg }}= \\
D \int \mathrm{d} \tau\left[\int_{-\infty}^{\infty} \frac{\mathrm{d} k}{2 \pi} \rho^{-1} \mathcal{C}\left(\frac{d}{d \tau}+i k\right) \exp \left(\frac{-\left(\frac{d}{d \tau}+i k\right) \rho^{-1}\left(\frac{d}{d \tau}+i k\right)}{\mathcal{M}^{2}}\right) 1\right]_{0} .
\end{gathered}
$$

Rescaling $k$ by $\mathcal{M}$ produces

$$
\begin{gathered}
(\Delta S)_{\text {reg }}=D \int \mathrm{d} \tau \int_{-\infty}^{\infty} \frac{\mathrm{d} k}{2 \pi}\left[\mathcal{M} \rho^{-1} \mathcal{C}\left(\frac{d}{d \tau}+i \mathcal{M} k\right) \times\right. \\
\left.\exp \left(\frac{k^{2}}{\rho}-i \frac{k}{\mathcal{M}}\left(\rho^{-1} \frac{d}{d \tau}+\frac{d}{d \tau} \rho^{-1}\right)-\frac{1}{\mathcal{M}^{2}} \frac{d}{d \tau} \rho^{-1} \frac{d}{d \tau}\right) 1\right]_{0} .
\end{gathered}
$$

Eq.(C.3) is in the form of Eq. (C.1) where $\mathcal{R}_{0}=k^{2} / \rho$. We use the Dyson-like expansion in Eq. C.1] and pick out the $\mathcal{M}$-independent term to arrive at

$$
(\Delta S)_{\mathrm{reg}}=
$$




$$
\begin{gathered}
-i D \int \mathrm{d} \tau \int_{-\infty}^{\infty} \frac{\mathrm{d} k}{2 \pi} \\
k \rho^{-1} \mathcal{C}\left\{\frac{d}{d \tau} \int_{0}^{1} \mathrm{~d} s \exp \left((1-s) \frac{k^{2}}{\rho}\right)\left(\rho^{-1} \frac{d}{d \tau}+\frac{d}{d \tau} \rho^{-1}\right) \exp \left(s \frac{k^{2}}{\rho}\right)\right. \\
+\int_{0}^{1} \mathrm{~d} s \exp \left((1-s) \frac{k^{2}}{\rho}\right)\left(\frac{d}{d \tau} \rho^{-1} \frac{d}{d \tau}\right) \exp \left(s \frac{k^{2}}{\rho}\right) \\
+k^{2} \int_{0}^{1} \mathrm{~d} u \int_{0}^{u} \mathrm{~d} s \exp \left((1-u) \frac{k^{2}}{\rho}\right)\left(\rho^{-1} \frac{d}{d \tau}+\frac{d}{d \tau} \rho^{-1}\right) \times \\
\left.\exp \left((u-s) \frac{k^{2}}{\rho}\right)\left(\rho^{-1} \frac{d}{d \tau}+\frac{d}{d \tau} \rho^{-1}\right) \exp \left(s \frac{k^{2}}{\rho}\right)\right\} .
\end{gathered}
$$

The first term in Eq.(C.4) is zero because it is a total derivative. To calculate the other two terms rotate to Euclidean space using $k \rightarrow-i k_{E}$. Then the expression $k^{2} / \rho \rightarrow-k_{E}^{2} / \rho$ in the exponents yields gaussian damping factors, so that the integrals are convergent. Even before evaluating the derivatives $\frac{d}{d \tau}$, it is clear that the integrand is an odd function of $k$ and hence produces a zero integral.

For the chiral Schwinger model, one starts with Eq.(9.41). Using momentum-space wave functions, the functional trace is

$$
\begin{gathered}
\Delta S=-i \int \mathrm{d}^{2} x \mathcal{C} \times \\
{\left[\int \frac{\mathrm{d}^{2} k}{(2 \pi)^{2}} \exp (-i k \cdot x)\left(\exp \left(\frac{R_{+}}{\mathcal{M}^{2}}\right)-\exp \left(\frac{R_{-}}{\mathcal{M}^{2}}\right)\right) \exp (i k \cdot x)\right]_{0},}
\end{gathered}
$$

Equation (C.2) is used to eliminate the $\exp ( \pm i k \cdot x)$ factors. Then, one scales the momentum $k$ by $\mathcal{M}$. The expression for $\Delta S$ becomes

$$
\Delta S=-i \int \mathrm{d}^{2} x \mathcal{C} \int \frac{\mathrm{d}^{2} k}{(2 \pi)^{2}}\left[\mathcal{M}^{2}\left(\exp \left(\tilde{R}_{+}\right)-\exp \left(\tilde{R}_{-}\right)\right) 1\right]_{0},
$$

where

$$
\tilde{R}_{ \pm}=-k^{\mu} k_{\mu}-\frac{-2 i k^{\mu} \partial_{\mu} \pm A_{-} k_{+}}{\mathcal{M}}+\frac{\partial^{\mu} \partial_{\mu} \pm i\left(\partial_{+} A_{-}\right) \pm i A_{-} \partial_{+}}{\mathcal{M}^{2}} .
$$

The parenthesis around $\left(\partial_{+} A_{-}\right)$indicates that $\partial_{+}$acts only on $A_{-}$.

The notation []$_{0}$ selects the term in Eq. [C.6) independent of $\mathcal{M}$. Hence, one expands in the factors in the exponentials proportional to $\mathcal{M}^{-1}$ and $\mathcal{M}^{-2}$. This results in two terms, $\Delta S_{1}$ from the leading Taylor series term in $\mathcal{M}^{-2}$, and $\Delta S_{2}$ from the second order term in $\mathcal{M}^{-1}: \Delta S=\Delta S_{1}+\Delta S_{2}$, where

$$
\Delta S_{1}=2 \int \mathrm{d}^{2} x \mathcal{C}\left(\partial_{+} A_{-}\right) \int \frac{\mathrm{d}^{2} k}{(2 \pi)^{2}} \exp \left(-k^{\mu} k_{\mu}\right)
$$




$$
\Delta S_{2}=i \int \mathrm{d}^{2} x \mathcal{C} \int \frac{\mathrm{d}^{2} k}{(2 \pi)^{2}} \exp \left(-k^{\mu} k_{\mu}\right) 2 i k^{\mu}\left(\partial_{\mu} A_{-}\right) k_{+} .
$$

Integrals are defined by analytic continuation using Wick rotation, that is, the $k_{0}$ integral is performed by going to Euclidean space. The line integral contained in $\int_{-\infty}^{\infty} \mathrm{d} k_{0}$ can be rotated counterclockwise by $90^{\circ}$. Then, one sets $k_{0}=-i k_{0}^{E}$ :

$$
\int_{-\infty}^{\infty} \mathrm{d} k_{0}=\int_{-i \infty}^{i \infty} \mathrm{d} k_{0}=-i \int_{\infty}^{-\infty} \mathrm{d} k_{0}^{E}=i \int_{-\infty}^{\infty} \mathrm{d} k_{0}^{E}
$$

Integrals are then convergent since

$$
\exp \left[-k^{2}\right]=\exp \left[+k_{0}^{2}-k_{1}^{2}\right] \rightarrow \exp \left[-k_{0}^{E 2}-k_{1}^{2}\right],
$$

provides a gaussian damping factor. The following integration table is obtained

$$
\int \frac{\mathrm{d}^{2} k}{(2 \pi)^{2}} \exp \left(-k^{\mu} k_{\mu}\right)\left\{\begin{array}{l}
1 \\
k_{0}^{2} \\
k_{1}^{2} \\
k_{0} k_{1}
\end{array}\right\}=\frac{i}{8 \pi}\left\{\begin{array}{l}
2 \\
-1 \\
1 \\
0
\end{array}\right\} .
$$

Hence,

$$
\begin{gathered}
\Delta S_{1}=\frac{2 i}{4 \pi} \int \mathrm{d}^{2} x \mathcal{C}\left(\partial_{+} A_{-}\right) \\
\Delta S_{2}=-\frac{i}{4 \pi} \int \mathrm{d}^{2} x \mathcal{C}\left(\partial_{+} A_{-}\right)
\end{gathered}
$$

Adding the two contributions,

$$
\Delta S=\frac{i}{4 \pi} \int \mathrm{d}^{2} x \mathcal{C}\left(\partial_{+} A_{-}\right)
$$

The factor $\partial_{+} A_{-}$can be written in Lorentz covariant form using

$$
\partial_{+} A_{-}=\partial_{0} A_{0}-\partial_{1} A_{1}-\partial_{0} A_{1}+\partial_{1} A_{0}=-\partial_{\mu} A^{\mu}+\varepsilon^{\mu \nu} \partial_{\mu} A_{\nu} .
$$

Substituting Eq.(C.14) into Eq.(C.13) produces the result in Eq.(9.43).

For the first-quantized bosonic string theory, we need to compute $\kappa_{r}$ of Eq.9.78). Using momentum-space eigenfunctions, it can be expressed as [121, 122]

$$
\kappa_{r}=\frac{t r}{2}\left[\int \frac{\mathrm{d}^{2} k}{(2 \pi)^{2}} \exp (-i k \cdot x) \exp \left(\frac{H_{r}}{\mathcal{M}^{2}}\right) \exp (i k \cdot x)\right]_{0},
$$


where $t r$ is a trace over two-by-two gamma-matrix space. In Eq.(C.15), $k \cdot x=$ $-k_{\tau} \tau+k_{\sigma} \sigma$. Using the definition of $H_{r}$ in Eq.9.76) and commuting $\exp (i k \cdot x)$ through the expression via Eq.(C.2), Eq.(C.15) becomes

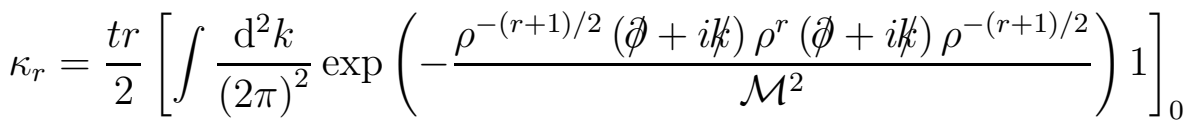

$$
\begin{aligned}
& =\frac{\operatorname{tr}}{2}\left[\mathcal{M}^{2} \int \frac{\mathrm{d}^{2} k}{(2 \pi)^{2}} \exp \left(-\frac{k^{2}}{\rho}-i \frac{A}{\mathcal{M}}+\frac{B}{\mathcal{M}^{2}}\right) 1\right]_{0},
\end{aligned}
$$

where we have performed the rescaling $k \rightarrow \mathcal{M} k$, and where

$$
\begin{gathered}
A \equiv \rho^{-(r+1) / 2}\left(\not \partial \rho^{r} \not k+\not k \rho^{r} \not \partial\right) \rho^{-(r+1) / 2}, \\
B \equiv-\rho^{-(r+1) / 2} \not \partial \rho^{r} \not \partial \rho^{-(r+1) / 2} .
\end{gathered}
$$

For the next step, we use the Dyson-like expansion in Eq.(C.1). Recalling that [ ] $]_{0}$ indicates that the $\mathcal{M}$-independent term is to be selected, $\kappa_{r}$ becomes a sum of two terms

$$
\kappa_{r}=B \text {-term }+A A \text {-term },
$$

where

$$
\begin{gathered}
A A \text {-term }=-\frac{t r}{2} \int \frac{\mathrm{d}^{2} k}{(2 \pi)^{2}} \int_{0}^{1} \mathrm{~d} u \int_{0}^{u} \mathrm{~d} s \times \\
\exp \left[-(1-u) \frac{k^{2}}{\rho}\right] A \exp \left[-(u-s) \frac{k^{2}}{\rho}\right] A \exp \left[-s \frac{k^{2}}{\rho}\right] 1,
\end{gathered}
$$

and

$$
B \text {-term }=\frac{t r}{2} \int \frac{\mathrm{d}^{2} k}{(2 \pi)^{2}} \int_{0}^{1} \mathrm{~d} s \exp \left[-(1-s) \frac{k^{2}}{\rho}\right] B \exp \left[-s \frac{k^{2}}{\rho}\right] 1 .
$$

In $A A$-term, carry out the differentiations $\not \partial$ in both $A$ operators using the definition of $A$ in Eq. (C.16) to obtain

$$
\begin{gathered}
A A \text {-term }=-\int \frac{\mathrm{d}^{2} k}{(2 \pi)^{2}} \int_{0}^{1} \mathrm{~d} u \int_{0}^{u} \mathrm{~d} s k^{2} \exp \left[-k^{2}\right]\left\{\frac{\partial^{n} \partial_{n} \rho}{\rho}\left(2 s k^{2}-1\right)+\right. \\
\left.\frac{\partial^{n} \rho \partial_{n} \rho}{\rho^{2}}\left(-\frac{1}{2}\left(r^{2}-5\right)-7 s k^{2}-u k^{2}+2 s u k^{4}\right)\right\},
\end{gathered}
$$

where another rescaling $k \rightarrow k \rho^{1 / 2}$ has been performed. Next, rotate the $k$ integration from Minkowski space to Euclidean space. The line integral contained in $\int_{-\infty}^{\infty} \mathrm{d} k_{\tau}$ can be rotated counterclockwise by $90^{\circ}$. One sets $k_{\tau}=-i k_{\tau}^{E}$ and uses Eq.(C.9) for 
$k_{0}=k_{\tau}$. The exponential factor $\exp \left[-k^{2}\right]=\exp \left[+k_{\tau}^{2}-k_{\sigma}^{2}\right]$ in $A A$-term becomes $\exp \left[-k_{\tau}^{E 2}-k_{\sigma}^{2}\right]$. One can then do the $s, u$ and $k$ integrations since the latter are now convergent. The result is

$$
A A \text {-term }=\frac{1}{4 \pi i}\left(\frac{\partial^{n} \partial_{n} \rho}{\rho} \frac{1}{6}-\frac{\partial^{n} \rho \partial_{n} \rho}{\rho^{2}}\left(\frac{r^{2}+1}{4}\right)\right) .
$$

The $B$-term is treated similarly. One carries out the differentiations $\not \partial$ in the $B$ operator and rescales $k$ by $\rho^{1 / 2}$ to arrive at

$$
\begin{aligned}
B \text {-term }= & \frac{t r}{2} \int \frac{\mathrm{d}^{2} k}{(2 \pi)^{2}} \int_{0}^{1} \mathrm{~d} s \exp \left[-k^{2}\right]\left\{\frac{\partial^{n} \partial_{n} \rho}{\rho}\left(s k^{2}-\frac{r+1}{2}\right)+\right. \\
& \left.\frac{\partial^{n} \rho \partial_{n} \rho}{\rho^{2}}\left(\frac{(3-r)(r+1)}{4}-3 s k^{2}+s^{2} k^{4}\right)\right\} .
\end{aligned}
$$

After rotating to Euclidean space, all integrations can be formed. The $B$-term is

$$
B \text {-term }=\frac{1}{4 \pi i}\left(\frac{\partial^{n} \partial_{n} \rho}{\rho} \frac{r}{2}+\frac{\partial^{n} \rho \partial_{n} \rho}{\rho^{2}}\left(\frac{r^{2}}{4}-\frac{r}{2}+\frac{1}{12}\right)\right) .
$$

The sum of the $A A$ - and $B$-terms in Eqs.(C.18) and (C.19) is the quoted result for $\kappa_{r}$ in Eq.(9.79). 


\section{References}

[1] E. S. Abers and B. W. Lee, Gauge Theories, Phys. Rep. C9 (1973) 1.

[2] J. Alfaro and P. H. Damgaard, Origin of Antifields in the Batalin-Vilkovisky Lagrangian Formalism, Nucl. Phys. B404 (1993) 751;

Generalized Lagrangian Master Equations, Phys. Lett. B334 (1994) 369.

[3] L. Alvarez-Gaumé and L. Baulieu, The Two Quantum Symmetries Associated with a Classical Symmetry, Nucl. Phys. B212 (1983) 255.

[4] D. Anselmi, Predictivity and Nonrenormalizability, SISSA preprint SISSA-14793-EP, (Sept., 1993), hep-th/9309085.

[5] S. Aoyama and S. Vandoren, The Batalin-Vilkovisky Formalism on Fermionic Kähler Manifolds, Mod. Phys. Lett. A8 (1993) 3773.

[6] C. Aragone and S. Deser, Consistency Problems in Hypergravity, Phys. Lett. 86B (1979) 161.

[7] O. Babelon, F. A. Schaposnik and C. M. Viallet, Quantization of Gauge Theories with Weyl Fermions, Phys. Lett. 177B (1986) 385.

[8] G. Bandelloni, Yang-Mills Cohomology in Four Dimensions, J. Math. Phys. 27 (1986) 2551; Nonpolynomial Yang-Mills Cohomology, J. Math. Phys. 28 (1987) 2775 .

[9] T. Banks, D. Friedan, E. Martinec, M. E. Peskin and C. R. Preitschopf, All Free String Theories Are Theories of Forms, Nucl. Phys. B274 (1986) 71.

[10] A. Barducci, R. Casalbouni and L. Lusanna, Supersymmetries and the Pseudoclassical Relativistic Electron, Nuovo Cim. 35A (1976) 377.

[11] G. Barnich, F. Brandt and M. Henneaux, Local BRST Cohomology in the Antifield Formalism: I. General Theorems, preprint ULB-th-94/06, NIKHEF-H 94-13, hep-th/9405109.

[12] G. Barnich, R. Constantinescu and P. Grégoire, BRST-Anti-BRST Antifield Formalism. The Example of the Freedman-Townsend Model, Phys. Lett. B293 (1992) 353.

[13] G. Barnich and M. Henneaux, Consistent Couplings Between Fields with a Gauge Freedom and Deformations of the Master Equation, Phys. Lett. B311 (1993) 123. 
[14] G. Barnich and M. Henneaux, Renormalization of Gauge Invariant Operators and Anomalies in Yang-Mills Theory, Phys. Rev. Lett. 72 (1994) 1588.

[15] F. Bastianelli, Ward Identities for Quantum Field Theories with External Fields, Phys. Lett. B263 (1991) 411.

[16] F. Bastianelli, A Locally Supersymmetric Action for the Bosonic String, Phys. Lett. B322 (1994) 340.

[17] I. A. Batalin, Quasigroup Construction and First Class Constraints, J. Math. Phys. 22 (1981) 1837.

[18] I. A. Batalin and E. S. Fradkin, A Generalized Canonical Formalism and Quantization of Reducible Gauge Theories, Phys. Lett. 122B (1983) 157.

[19] I. A. Batalin and E. S. Fradkin, Operator Quantization and Abelization of Dynamical Systems Subject to First-Class Constraints, Riv. Nuov. Cim. 9 (1986) 1.

[20] I. A. Batalin, P. M. Lavrov and I. V. Tyutin, Covariant Quantization of Gauge Symmetries in the Framework of Extended BRST Symmetry, J. Math. Phys. 31 (1990) 1487;

An Sp(2)-Covariant Quantization of Gauge Theories with Linearly Dependent Generators, J. Math. Phys. 32 (1991) 532.

[21] I. A. Batalin and I. V. Tyutin, On the Multilevel Generalization of the FieldAntifield Formalism, Mod. Phys. Lett. A8 (1993) 3673.

[22] I. A. Batalin and I. V. Tyutin, On Possible Generalizations of Field-Antifield Formalism, Int. J. Mod. Phys. A8 (1993) 2333.

[23] I. A. Batalin and G. A. Vilkovisky, Relativistic S-Matrix of Dynamical Systems with Boson and Fermion Constraints, Phys. Lett. 69B (1977) 309.

[24] I. A. Batalin and G. A. Vilkovisky, Gauge Algebra and Quantization, Phys. Lett. 102B (1981) 27.

[25] I. A. Batalin and G. A. Vilkovisky, Feynman Rules for Reducible Gauge Theories, Phys. Lett. 120B (1983) 166.

[26] I. A. Batalin and G. A. Vilkovisky, Quantization of Gauge Theories with Linearly Dependent Generators, Phys. Rev. D28 (1983) 2567; Errata: D30 (1984) 508. 
[27] I. A. Batalin and G. A. Vilkovisky, Closure of the Gauge Algebra, Generalized Lie Algebra Equations and Feynman Rules, Nucl. Phys. B234 (1984) 106.

[28] I. A. Batalin and G.A.Vilkovisky, Existence Theorem for Gauge Algebra, J. Math. Phys. 26 (1985) 172.

[29] C. Batlle, Teories de Camps de Cordes, Thesis, Universitat de Barcelona (1988).

[30] C. Batlle and J.Gomis, Lagrangian and Hamiltonian BRST Structures of the Antisymmetric Tensor Gauge Theory, Phys. Rev. D38 (1988) 1169.

[31] C. Batlle, J. Gomis, J. París and J. Roca, Lagrangian and Hamiltonian BRST Formalisms, Phys. Lett. B224 (1989) 288;

Field-Antifield Formalism and Hamiltonian BRST Approach, Nucl.Phys. B329 (1990) 139.

[32] L. Baulieu, Perturbative Gauge Theories, Phys. Rep. 129 (1985) 1.

[33] L. Baulieu, M. Bellon, S. Ouvry and J.-C. Wallet, Batalin-Vilkovisky Analysis of Supersymmetric Systems, Phys. Lett. B252 (1990) 387.

[34] L. Baulieu, E. Bergshoeff and E. Sezgin, Open BRST Algebras, Ghost Unification and String Field Theory, Nucl. Phys. B307 (1988) 348.

[35] L. Baulieu and I.M. Singer, Topological Yang-Mills Symmetry, Nucl.Phys. B (Proc. Suppl) 5B (1988) 12.

[36] C. Becchi, A. Rouet and R. Stora, The Abelian Higgs Kibble Model, Unitarity of the S-Operator, Phys. Lett. 52B (1974) 344;

Renormalization of the Abelian Higgs-Kibble Model, Comm. Math.Phys. 42 (1975) 127;

Renormalization of Gauge Theories, Ann. Phys. 98 (1976) 287.

[37] F. A. Berends, J. W. van Holten, P. van Nieuwenhuizen and B. de Wit, On Spin 5/2 Gauge Fields, Phys. Lett. 83B (1979) 188.

[38] F. A. Berezin, The Method of Second Quantization, (Academic Press, New York, 1966).

[39] F. A. Berezin, Introduction to Superanalysis, Mathematical Physics and Applied Mathematics, no 9, (Reidel, Dordrecht, 1987).

[40] F. A. Berezin and D. A. Leites, Supermanifolds, Sov. Math. Dokl. 16 (1975) 1218. 
[41] F. A. Berezin and M.S. Marinov, Particle Spin Dynamics as the Grassmann Variant of Classical Mechanics, Ann. Phys. 104 (1977) 336.

[42] E. Bergshoeff, J. de Boer, M. de Roo and T. Tjin, On the Cohomology of the Non-Critical W String, Nucl. Phys. B420 (1994) 379.

[43] E. Bergshoeff and R. Kallosh, Unconstrained BRST for Superparticles, Phys. Lett. B240 (1990) 105.

[44] E. Bergshoeff, R. Kallosh and A. Van Proeyen, Superparticle Actions and Gauge Fixings, Clas. Quantum Grav. 9 (1992) 321.

[45] E. Bergshoeff, A. Sevrin and X. Shen, A Derivation of the BRST Operator for Non-Critical W-Strings, Phys. Lett. B296 (1992) 95.

[46] N. Berkovits, M. T. Hatsuda and W. Siegel, The Big Picture, Nucl. Phys. B371 (1992) 434.

[47] M. Bershadsky, S. Cecotti, H. Ooguri and C. Vafa, Kodaira-Spencer Theory of Gravity and Exact Results for Quantum String Amplitudes, Harvard Univ. preprint HUTP-93-A025, (Sept, 1993), hep-th/9309140.

[48] M. Bershadsky, W. Lerche, D. Nemeschansky and N.P. Warner, A BRST Operator for Non-Critical Strings, Phys. Lett. B292 (1993) 35;

Extended $N=2$ Superconformal Structure of Gravity and W-Gravity Coupled to Matter, Nucl. Phys. B401 (1993) 304.

[49] D. Birmingham, M. Blau, M. Rakowski and G. Thompson, Topological Field Theory, Phys. Rep. 209 (1991) 129.

[50] D. Birmingham, M. Rakowski and G. Thompson, BRST Quantization of Topological Field Theories, Nucl. Phys. B315 (1989) 577.

[51] M. Blagojević and B.Sazdović, Off-Shell BRST Quantization of Reducible Gauge Theories, Phys. Lett. B223 (1989) 325.

[52] A. Blasi and G.Bandelloni, Antisymmetric Tensor Gauge Theory, Clas. Quantum Grav. 10, (1993) 1249.

[53] K. Bleuler, A New Method for the Treatment of Longitudinal and Scalar Photons, Helv. Phys. Acta 23 (1950) 567.

[54] R. Bluhm and S. Samuel, The Off-Shell Koba-Nielsen Formula, Nucl. Phys. B323 (1989) 337. 
[55] R. Bluhm and S. Samuel, Off-Shell Conformal Field Theory at the One-Loop Level, Nucl. Phys. B325 (1989) 275.

[56] M. Bochicchio, String Field Theory in the Siegel Gauge, Phys. Lett. 188B (1987) 330;

Gauge Fixing for the Field Theory of the Bosonic String, Phys. Lett. 193B (1987) 31.

[57] L. Bonora and P. Cotta-Ramusino, Some Remarks on BRST Transformations, Anomalies and the Cohomology of the Lie Algebra of the Group of Gauge Transformations, Comm. Math. Phys. 87 (1983) 589.

[58] A. Borel, Sur la Cohomologie des Espaces Fibres Principaux et Espaces Homogenes de Groupes de Lie Compacts, Ann. Math. 57 (1953) 115.

[59] P. Bouwknegt, J. McCarthy and K. Pilch, BRST Analysis of Physical States for 2-d Gravity Coupled to $c \leq 1$ Matter, Comm. Math. Phys. 145 (1992) 541.

[60] P. Bouwknegt, J. McCarthy and K. Pilch, Semi-infinite Cohomology of W Algebras, Lett. Math. Phys. 29 (1993) 91.

[61] N. R.F. Braga and H. Montani, Batalin-Vilkovisky Lagrangian Quantization of the Chiral Schwinger Model, Phys. Lett. B264 (1991) 125.

[62] N.R.F. Braga and H. Montani, The Wess-Zumino Term in the Field-Antifield Formalism, Int. J. Mod. Phys. A8 (1993) 2569.

[63] N.R.F. Braga and H. Montani, BRST Quantization of the Chiral Schwinger Model in the Extended Field-Antifield Space, Phys. Rev. D49 (1994) 1077.

[64] F. Brandt, Antifield Dependence of Anomalies, Phys. Lett. B320 (1994) 57.

[65] L. Brink, S. Deser, B. Zumino, P. di Vecchia and P. Howe, Local Supersymmetry for Spinning Particles, Phys. Lett. 64B (1977) 435.

[66] R. Brooks, D. Montano and J. Sonnenschein, Gauge Fixing and Renormalization in Topological Quantum Field Theory, Phys. Lett. B214 (1988) 91.

[67] E. Cremmer and J. Scherk, Spontaneous Dynamical Breaking of Gauge Symmetry in Dual Models, Nucl. Phys. B72 (1974) 117.

[68] E. Cremmer, A.Schwimmer and C. Thorn, The Vertex Function in Witten's Formulation of String Field Theory, Phys. Lett. B179 (1986) 57. 
[69] G. Curci and R. Ferrari, Slavnov Transformations and Supersymmetry, Phys. Lett. 63B (1976) 91.

[70] G. Curci and R. Ferrari, An Alternative Approach to the Proof of Unitarity for Gauge Theories, Nuovo Cim. 35A(1976) 273.

[71] O.F. Dayi, Odd Time Formulation of the Batalin-Vilkovisky Method of Quantization, Mod. Phys. Lett. A4 (1989) 361.

[72] O.F. Dayi, BV and BFV Formulation of a Gauge Theory of Quadratic Lie Algebras in 2-D and a Construction of $W_{3}$ Topological Gravity, Tubitak Research Institute preprint, (Jan., 1994), hep-th/9401148.

[73] S. P. de Alwis, M. T. Grisaru and L. Mezincescu, Quantization and Unitarity in Antisymmetric Tensor Gauge Theories, Nucl. Phys. B303 (1988) 57.

[74] J. de Boer and J. Goeree, The Effective Action of $W_{3}$ to All Orders, Nucl. Phys. B401 (1993) 348.

[75] F. De Jonghe, The Batalin-Vilkovisky Lagrangian Quantization Scheme: With Applications to the Study of Anomalies in Gauge Theories, Leuven Univ. preprint, (Dec., 1993), hep-th/9403143.

[76] F. De Jonghe, Schwinger-Dyson BRST Symmetry and the Equivalence of Hamiltonian and Lagrangian Quantisation, Phys. Lett. B316 (1993) 503.

[77] F. De Jonghe, R. Siebelink and W. Troost, Hiding Anomalies, Phys. Lett. B306 (1993) 295.

[78] F. De Jonghe, R. Siebelink, W. Troost, S. Vandoren, P. van Nieuwenhuizen and A. Van Proeyen, The Regularized BRST Jacobian of Pure Yang-Mills Theory, Phys. Lett. B289 (1992) 354.

[79] F. De Jonghe and S. Vandoren, Construction of Topological Field Theories Using the Batalin-Vilkovisky Quantization Scheme, Phys. Lett. B324 (1994) 328.

[80] S. Deser, P. K. Townsend and W. Siegel, Higher Rank Representations of Lower Spin, Nucl. Phys. B184 (1981) 333.

[81] B. de Wit and J. W. van Holten, Covariant Quantization of Gauge Theories with Open Algebra, Phys. Lett. B79 (1979) 389. 
[82] B. DeWitt, Dynamical Theory of Groups and Fields, in Relativity, Groups and Topology, Les Houches Summer School, Session XIII, edited by C. DeWitt and B. DeWitt, (Gordon Breach, 1964).

[83] B. DeWitt, Quantum Theory of Gravity. I. The Canonical Theory, Phys. Rev. 160 (1967) 1113;

Quantum Theory of Gravity. II. The Manifestly Covariant Theory, Phys. Rev. 162 (1967) 1195;

Quantum Theory of Gravity.III. Applications of the Covariant Theory, Phys. Rev. 162 (1967) 1239.

[84] B. S. DeWitt, The Spacetime Approach to Quantum Field Theory, in Relativity, Groups and Topology, II, Les Houches Summer School, session XL, edited by B. S. DeWitt and R. Stora, (North-Holland, Amsterdam, 1984).

[85] B. S. DeWitt, Supermanifolds, (Cambridge Univ. Press, Cambridge, 1984).

[86] A. H. Diaz, The Nonabelian Antisymmetric Tensor Field Revisited, Phys. Lett. B203 (1988) 408.

[87] A. Diaz, W. Troost, P. van Nieuwenhuizen and A. Van Proeyen, Understanding Fujikawa Regulators from Pauli-Villars Regularization of Ghost Loops, Int. J. Mod. Phys. A4 (1989) 3959.

[88] P. A. M. Dirac, Lectures on Quantum Mechanics, (Yeshiva University, New York, 1964).

[89] J.A. Dixon, Cohomology and Renormalization of Gauge Theories I, II,III,IV, unpublished (1976-1979); Comm. Math. Phys. 139 (1991) 495.

[90] S. Donaldson, An Application of Gauge Theory to the Topology of Four Manifolds, J. Diff. Geom. 18 (1983) 279;

Polynomial Invariants of Smooth Four-Manifolds, Topology 29 (1990) 257.

[91] A. Dresse, J.M. L.Fisch, P. Grégoire and M.Henneaux, Equivalence of the Hamiltonian and Lagrangian Path Integrals for Gauge Theories, Nucl. Phys. B354 (1991) 191.

[92] M. Dubois-Violette, M. Henneaux, M. Talon and C. M. Viallet, Some Results on Local Cohomologies in Field Theory, Phys. Lett. B267 (1991) 81. 
[93] M. Dubois-Violette, M. Talon and C. M. Viallet, B. R. S. Algebras. Analysis of the Consistency Equations in Gauge Theory, Comm. Math. Phys. 102 (1985) 105.

[94] F. J. Dyson, The S-Matrix in Quantum Electrodynamics, Phys. Rev. 75 (1949) 1736.

[95] R. J.Eden, P. V. Landshoff, D. I. Olive and J. C. Polkinghorne, The Analytic SMatrix, (Cambridge University Press, Cambridge, UK, 1966).

[96] T. Eguchi, P. B. Gilkey and A. J. Hanson, Gravitation, Gauge Theories and Differential Geometry, Phys. Rep. 66 (1980) 213.

[97] L. D. Faddeev, Operator Anomaly for the Gauss Law, Phys. Lett. 145B (1984) 81.

[98] L. D. Faddeev and V. N. Popov, Feynman Diagrams for the Yang-Mills Field, Phys. Lett. 25B (1967) 29.

[99] L. D. Faddeev and S. L. Shatashvili, Algebraic and Hamiltonian Methods in the Theory of Non-Abelian Anomalies, Theor. Math. Phys. 60 (1984) 770;

Realization of the Schwinger Term in the Gauss Law and the Possibility of Correct Quantization of a Theory with Anomalies, Phys. Lett. 167B (1986) 225 .

[100] L. D. Faddeev and A. A. Slavnov, Gauge Fields: Introduction to Quantum Theory, (Benjamin-Cummings, Reading, MA, 1980).

[101] R. P. Feynman, Quantum Theory of Gravitation, Acta Phys. Pol. 24 (1963) 697.

[102] R.P. Feynman and A.R.Hibbs, Quantum Mechanics and Path Integrals, (McGraw-Hill, New York, 1965).

[103] J.M. L. Fisch, On the Batalin-Vilkovisky Antibracket-Antifield BRST Formalism and Its Applications, Univ. Libre de Bruxelles preprint ULB-TH2-90-01, (Jan., 1990).

[104] J. M. L. Fisch and M. Henneaux, The Antifield-Antibracket Formalism for Constrained Hamiltonian Systems, Phys. Lett. B226 (1989) 80.

[105] J. M. L. Fisch and M. Henneaux, Homological Perturbation Theory and the Algebraic Structure of the Antifield-Antibracket Formalism for Gauge Theories, Comm. Math. Phys. 128 (1990) 627. 
[106] J. M. L. Fisch, M. Henneaux, J. Stasheff and C. Teitelboim, Existence, Uniqueness and Cohomology of the Classical BRST Charge with Ghosts for Ghosts, Comm. Math. Phys. 120 (1989) 379.

[107] H. Flanders, Differential Forms, (Academic Press, 1963).

[108] E. S. Fradkin and T. E. Fradkina, Quantization of Relativistic Systems with Boson and Fermion First- and Second-Class Constraints, Phys. Lett. 72B (1978) 343.

[109] E.S. Fradkin and G.A. Vilkovisky, Quantization of Relativistic Systems with Constraints, Phys. Lett. 55B (1975) 224.

[110] E. S. Fradkin and G. A. Vilkovisky, Unitarity of S-Matrix in Gravidynamics and General Covariance in Quantum Domain, Nuovo Cim. Lett. 13 (1975) 187.

[111] D. Z. Freedman, S. Giddings, J. Shapiro and C. B. Thorn, The Nonplanar OneLoop Amplitude in Witten's String Field Theory, Nucl. Phys. B298 (1988) 253.

[112] D. Z. Freedman and P. K. Townsend, Antisymmetric Tensor Gauge Theories and Non-Linear $\sigma$-Models, Nucl. Phys. B177 (1981) 282.

[113] D.Z. Freedman and P. van Nieuwenhuizen, Properties of Supergravity Theory, Phys. Rev. D14 (1976) 912.

[114] D. Z. Freedman, P. van Nieuwenhuizen and S. Ferrara, Progress Toward a Theory of Supergravity, Phys. Rev. D13 (1976) 3214.

[115] I. B. Frenkel, H. Garland and G. J.Zuckerman, Semi-Infinite Cohomology and String Theory, Proc. Natl. Acad. Sci. 83 (1986) 8442.

[116] S. A. Frolov and A.A.Slavnov, Quantization of Non-Abelian Antisymmetric Tensor Field, Theor. Math. Phys. 75 (1988) 470.

[117] S. A. Frolov and A. A. Slavnov, Physical Subspace Norm in Hamiltonian BRSTQuantization, Phys. Lett. B218 (1989) 461.

[118] S. A. Frolov and A. A. Slavnov, Construction of the Effective Action for General Gauge Theories Via Unitarity, Nucl. Phys. B347 (1990) 333.

[119] S. Frolov and A.Slavnov, Lagrangian BRST Quantization and Unitarity, Theor. Math. Phys. 85 (1990) 1237. 
J. Gomis, J. París and S. Samuel - Antibracket, Antifields and ...

[120] K. Fujikawa, Path-Integral Measure for Gauge-Invariant Fermion Theories, Phys. Rev. Lett. 42 (1979) 1195;

Path Integral for Gauge Theories with Fermions, Phys. Rev. D21 (1980) 2848.

[121] K. Fujikawa, Path Integral of Relativistic Strings, Phys. Rev. D25 (1982) 2584.

[122] K. Fujikawa, T. Inagaki and H. Suzuki, BRS Current and Related Anomalies in Two Dimensional Gravity and String Theories, Nucl. Phys. B332 (1990) 499.

[123] S. J. Gates Jr., M. T. Grisaru， U. Lindström， M. Roček， W. Siegel， P. van Nieuwenhuizen and A.E.van de Ven, Lorentz-Covariant Quantization of the Heterotic Superstring, Phys. Lett. B225 (1989) 44.

[124] S. J. Gates Jr., M. T. Grisaru, M. Roček and W. Siegel, Superspace or One Thousand and One Lessons in Supersymmetry, (Benjamin/Cummings, Reading, MA, 1983).

[125] M. Gerstenhaber, The Cohomology Structure of an Associative Ring, Ann. Math. 78 (1962) 267.

[126] M. Gerstenhaber, On the Deformation of Rings and Algebras, Ann. Math. 79 (1964) 59.

[127] E. Getzler, Batalin-Vilkovisky Algebras and Two-Dimensional Topological Field Theories, Comm. Math. Phys. 159 (1994) 265.

[128] S. Giddings, The Veneziano Amplitude from Interacting String Field Theory, Nucl. Phys. B278 (1986) 242

[129] S. L. Glashow, Partial Symmetries of Weak Interactions, Nucl. Phys. 22 (1961) 579 .

[130] J. Gomis and J. París, Unitarity and the Field-Antifield Formalism, Nucl. Phys. B368 (1992) 311.

[131] J. Gomis and J.París, Field-Antifield Formalism for Anomalous Gauge Theories, Nucl. Phys. B395 (1993) 288.

[132] J. Gomis and J.París, Perturbation Theory and Locality in the Field-Antifield Formalism, J. Math. Phys. 34 (1993) 2132.

[133] J. Gomis and J.París, Anomalies and Wess-Zumino Terms in an Extended Regularized Field-Antifield Formalism, Nucl.Phys. B431 (1994) 378, hepth/9401161. 
[134] J. Gomis and J.París, Anomalous Gauge Theories Within the Field-Antifield Formalism, in Proc. of the International EPS Conference on High Energy Physics, HEP93, Marseille, (July, 1993), p. 101-102.

[135] J. Gomis, J. París, K. Rafanelli and J. Roca, BRST and Anti-BRST Symmetries for the Spinning Particle, Phys. Lett. B246 (1990) 435.

[136] J.Gomis, J.París and J.Roca, BRST Structures of the Spinning String, Nucl. Phys. B339 (1990) 711.

[137] J. Gomis and J. Roca, The Anti-BRST Symmetry in the Field-Antifield Formalism, Nucl. Phys. B343 (1990) 152.

[138] T.Goto, Relativistic Quantum Mechanics of One Dimensional Mechanical Continuum as Subsidiary Condition of Dual Resonance Model, Prog. Theor. Phys. 46 (1971) 1560.

[139] M. B. Green and C. M. Hull, Covariant Quantum Mechanics of the Superstring, Phys. Lett. B225 (1989) 57.

[140] G. V. Grigorian, R.P. Grigorian and I. V.Tyutin, Equivalence of Lagrangian and Hamiltonian BRST Quantizations: Systems with First Class Constraints, Sov. J. Nucl. Phys. 50 (1991) 1058.

[141] G. V. Grigorian, R. P. Grigorian and I. V. Tyutin, Equivalence of Lagrangian and Hamiltonian BRST Quantizations: the General Case, Nucl. Phys. B379 (1992) 304.

[142] D. Gross and A. Jevicki, Operator Formulation of Interacting String Field Theory (I), Nucl. Phys. B283 (1987) 1.

[143] D. Gross and A. Jevicki, Operator Formulation of Interacting String Field Theory (II), Nucl. Phys. B287 (1987) 225.

[144] S. N. Gupta, Theory of Longitudinal Photons in Quantum Electrodynamics, Proc. Phys. Soc. A63 (1950) 681.

[145] K. Harada and I. Tsutsui, On the Path-Integral Quantization of Anomalous Gauge Theories, Phys. Lett. B183 (1987) 311.

[146] H. Hata, Construction of the Quantum Action for Path Integral Quantization of String Field Theory, Nucl. Phys. B339 (1990) 663. 
J. Gomis, J. París and S. Samuel - Antibracket, Antifields and ...

[147] H. Hata, "Theory of Theories" Approach to String Theory, Phys. Rev. D50 (1994) 4079.

[148] H. Hata, T. Kugo and N. Ohta, Skew-Symmetric Tensor Gauge Field Theory Dynamically Realized in the QCD U(1) Channel, Nucl. Phys. B178 (1981) 527.

[149] H. Hata and B.Zwiebach, Developing the Covariant Batalin-Vilkovisky Approach to String Field Theory, Ann. Phys. 229 (1994) 177.

[150] M. Henneaux, Hamiltonian Form of the Path Integral for Theories with a Gauge Freedom, Phys. Rep. 126 (1985) 1.

[151] M. Henneaux, On the Algebraic Structure of the BRST Symmetry, Lectures given at Banff Summer School in Theoretical Physics on Physics, Geometry and Topology, in Banff NATO ASI 1989, p.81-104.

[152] M. Henneaux, Lectures on the Antifield-BRST Formalism for Gauge Theories, Nucl. Phys. B (Proc. Suppl.) 18A (1990) 47.

[153] M. Henneaux, Elimination of the Auxiliary Fields in the Antifield Formalism, Phys. Lett. B238 (1990) 299.

[154] M. Henneaux, Spacetime Locality of the BRST Formalism, Comm. Math. Phys. 140 (1991) 1.

[155] M. Henneaux, Geometric Interpretation of the Quantum Master Equation in the BRST Anti-BRST Formalism, Phys. Lett. B282 (1992) 372.

[156] M. Henneaux, Remarks on the Renormalization of Gauge Invariant Operators in Yang-Mills Theory, Phys. Lett. B313 (1993) 35, (Erratum Phys. Lett. B316 (1993) 633).

[157] M. Henneaux and C. Teitelboim, Quantization of Gauge Systems, (Princeton University Press, Princeton, 1992).

[158] P. Horava, Space Time Diffeomorphism and Topological $W_{\infty}$ in Two Dimensional Topological String Theory, Nucl. Phys. B414 (1994) 485.

[159] P. S. Howe, U. Lindström and P. White, Anomalies and Renormalization in the BRST-BV Framework, Phys. Lett. B246 (1990) 430.

[160] C.M.Hull, The BRST and Anti-BRST Invariant Quantization of General Gauge Theories, Mod. Phys. Lett. A5 (1990) 1871. 
[161] C. M. Hull, Matter-Dependent W-Gravity Anomalies of Non-Linearly Realised Symmetries, Phys. Lett. B265 (1991) 347.

[162] C. M. Hull, W Gravity Anomalies with Ghost Loops and Background Charges, Int. J. Mod. Phys. A8 (1993) 2419.

[163] S. Hwang, Covariant Quantization of the String in Dimensions D $\leq 26$ Using a Becchi-Rouet-Stora Formulation, Phys. Rev. D28 (1983) 2614.

[164] H. Ikemori, Extended Form Method of Antifield BRST Formalism for Topological Quantum Field Theories, Clas. Quantum Grav. 10, (1993) 233.

[165] C. Itzykson and J. B. Zuber, Quantum Field Theory, (McGraw-Hill, New York, 1980).

[166] R. Jackiw and R. Rajaraman, Vector-Meson Mass Generation by Chiral Anomalies, Phys. Rev. Lett. 54 (1985) 1219.

[167] S. D. Joglekar and B. W. Lee, Gauge Theory of Renormalization of Gauge Invariant Operators, Ann. Phys. 97 (1976) 160.

[168] M. Kaku, Introduction to Superstrings, (Springer-Verlag, New York, 1988).

[169] M. Kaku, Geometrical Derivation of String Field Theory from First Principles: Closed Strings and Modular Invariance, Phys. Rev. D38 (1988) 3052; Nonpolynomial Closed-String Field Theory, Phys. Rev. D41 (1990) 3734.

[170] M. Kaku and J.Lykken, Modular Invariant Closed String Field Theory, Phys. Rev. D38 (1988) 3067.

[171] M. Kalb and P. Ramond, Classical Direct Interstring Action, Phys. Rev. D9 (1974) 2273.

[172] R. E. Kallosh, Modified Rules in Supergravity, Nucl. Phys. B141 (1978) 141.

[173] R. Kallosh, Super-Poincaré Covariant Quantized GS Heterotic String as a Free Conformal Theory, Phys. Lett. B224 (1989) 273.

[174] M. Kato and K. Ogawa, Covariant Quantization of String Based on BRS Invariance, Nucl. Phys. B212 (1983) 443.

[175] O. M. Khudaverdian, Geometry of Superspace with Even and Odd Brackets, J. Math. Phys. 32 (1991) 1934. 
J. Gomis, J. París and S. Samuel - Antibracket, Antifields and ...

[176] O. M. Khudaverdian and A.P. Nersesian, On the Geometry of the BatalinVilkovisky Formalism, Mod. Phys. Lett. A8 (1993) 2377.

[177] T. W. Kibble, Lorentz Invariance and the Gravitational Field, J. Math. Phys. 2 (1961) 212.

[178] T. Kimura, Antisymmetric Tensor Gauge Field in General Covariant Gauges, Prog. Theo. Phys. 64 (1980) 357;

Quantum Theory of Antisymmetric Higher Rank Tensor Gauge Field in Higher Rank Dimensional Space-Time, Prog. Theo. Phys. 65 (1981) 338.

[179] J.L. Koszul, Sur un Type d'Algébres Differéntielles en Raport avec la Transgression, Bull. Soc. Math. France 78 (1950) 5.

[180] T. Kugo, H. Kunitomo and K. Suehiro, Non-Polynomial Closed String Field Theory, Phys. Lett. B226 (1989) 48.

[181] T. Kugo and I. Ojima, Manifestly Covariant Canonical Formalism of Yang-Mills Theories, Physical State Subsidiary Conditions and Physical S-Matrix Unitarity, Phys. Lett. B73 (1978) 459.

[182] T. Kugo and I. Ojima, Local Covariant Operator Formalism of Non-Abelian Gauge Theories and Quark Confinement Problem, Prog. Theor. Phys. Suppl. 66 (1979) 1.

[183] T. Kugo, and K. Suehiro, Nonpolynomial Closed String Field Theory: Action and Gauge Invariance, Nucl. Phys. B337 (1990) 343.

[184] T. Kugo and S. Uehara, General Procedure of Gauge Fixing Based on BRS Invariance Principle, Nucl. Phys. B197 (1982) 378.

[185] J.M.F. Labastida and M.Pernici, A Gauge Invariant Action in Topological Quantum Field Theory, Phys. Lett. B212 (1988) 56.

[186] P. M. Lavrov and I. V. Tyutin, Effective Action in Gauge Theories of a General Form, Sov. J. Nucl. Phys. 41 (1985) 1049.

[187] B. W. Lee, Gauge Theories, in Methods in Field Theory, Les Houches Summer School 1975, edited by R. Balian and J.Zinn-Justin (North-Holland, Amsterdam, 1976).

[188] D. A. Leites, Introduction to the Theory of Supermanifolds, Russ. Math. Surv. 35 (1980) 1. 
[189] B.H.Lian and G.J.Zuckerman, New Perspectives on the BRST-Algebraic Structure of String Theory, Comm. Math. Phys. 154 (1993) 613.

[190] U. Lindström, M. Roček, W. Siegel, P. van Nieuwenhuizen and A. E. van de Ven, Lorentz-Covariant Quantization of the Superparticle, Phys. Lett. B224 (1989) 285 ;

Gauge-Fixing Redundant Symmetries in the Superparticle, Phys. Lett. B228 (1989) 53.

[191] C. Lucchesi, O.Piguet and S.P.Sorella, Renormalization and Finiteness of Topological BF Theories, Nucl. Phys. B395 (1993) 325.

[192] J. Lykken and S. Raby, Non-Commutative Geometry and the Closed Bosonic String, Nucl. Phys. B278 (1986) 256.

[193] N. Maggiore and S.P.Sorella, Perturbation Theory for Antisymmetric Tensor Fields in Four-Dimensions, Int. J. Mod. Phys. A8 (1993) 929.

[194] R. Marnelius, General State Spaces for BRST Quantizations, Nucl. Phys. B391 (1993) 621.

[195] R. Mohayee, C. N. Pope, K. S. Stelle and K. W.Xu, Canonical BRST Quantization of World Sheet Gravities, Texas A\&M preprint CTP-TAMU-18-94, (April, 1994), hep-th/9404170.

[196] N. Nakanishi and I. Ojima, Covariant Operator Formalism of Gauge Theories and Quantum Gravity, World Scientific Lecture Notes, Vol. 27 (1990).

[197] Y. Nambu, Duality and Hadrodynamics, Lectures at the Copenhagen Summer Symposium, (1970), published in Strings, Lattice Gauge Theory, High Energy Phenomenology, Proc. of the Winter School Panchgani, edited by V. Singh and S. R. Wadia, (World Scientific, 1987).

[198] A. P. Nersesian, On Geometry of Supermanifolds with Odd and Even Kahlerian Structures, Theor. Math. Phys. 96 (1993) 866.

[199] N. K. Nielsen, Ghost Counting in Supergravity, Nucl. Phys. B140 (1978) 494.

[200] N. K. Nielsen, BRS Invariance of Supergravity in a Gauge Involving an Extra Ghost, Phys. Lett. 103B (1981) 197.

[201] Kh.S. Nirov and A. V. Razumov, Field-Antifield and BFV Formalisms for Quadratic Systems with Open Gauge Algebras, Int. J. Mod.Phys. A7 (1992) 5719 . 
[202] I. Ojima, Another BRS Transformation, Prog. Theo. Phys. 64 (1981) 625.

[203] C. Ordóñez, J. París, J. M. Pons and R. Toldrà, BV Analysis for Covariant and Noncovariant Actions, Phys. Rev. D48 (1993) 3818.

[204] C. Ordóñez, J. M. Pons and R. Toldrà, Unitarity and Batalin-Vilkovisky Path Integral Quantization for Gauge Theories, Phys. Lett. B302 (1993) 423.

[205] F. R. Ore, Jr. and P. van Nieuwenhuizen, Local BRST Symmetry and the Geometry of Gauge-Fixing, Nucl. Phys. B204 (1982) 317.

[206] J. París, Anomalies i Unitarietat en el Formalisme de Camps i Anticamps, Thesis, Universitat de Barcelona (1992).

[207] J.París, Faddeev-Popov Method for Anomalous Quasigroups, Phys. Lett. B300 (1993) 104.

[208] J.E.Paton and H.-M.Chan, Generalized Veneziano Model with Isospin, Nucl. Phys. B10 (1969) 516.

[209] M.Penkava and A.Schwarz, On Some Algebraic Structure Arising in String Theory, UC Davis preprint PRINT-93-0001, (Dec., 1992), hep-th/9212072.

[210] A. M. Polyakov, Quantum Geometry of Bosonic Strings, Phys. Lett. 103B (1981) 207.

[211] M. Roček, W.Siegel, P. van Nieuwenhuizen and A.E. van de Ven, Covariant Superparticle Quantization in a Super Maxwell Background, Phys. Lett. B227 (1989) 87.

[212] M. Saadi and B.Zwiebach, Closed String Theory from Polyhedra, Ann. Phys. 192 (1989) 213.

[213] A. Salam, Weak and Electromagnetic Interactions, in Elementary Particle Theory, edited by N.Svartholm, (Almquist and Forlag, Stockholm, 1968), p.367377 .

[214] S. Samuel, The Physical and Ghost Vertices in Witten's String Field Theory, Phys. Lett. B181 (1986) 255.

[215] S. Samuel, Introduction to String Field Theory, in Strings and Superstrings, edited by J.P. Mittelbrunn, M. Ramón-Medrano and G. S. Rodero, El Escorial, Spain, June, 1987, (World Scientific, Singapore, 1988); 
Also see Appendices A-C of Mathematical Formulation of Witten's Superstring Field Theory, Nucl. Phys. B296 (1988) 187.

[216] S. Samuel, Covariant Off-Shell String Amplitudes, Nucl. Phys. B308 (1988) 285 ;

Off-Shell String Physics from Conformal Field Theory, Nucl. Phys. B308 (1988) 317.

[217] S. Samuel, Solving the Open Bosonic String in Perturbation Theory, Nucl. Phys. B341 (1990) 513.

[218] A. Schwarz, Geometry of Batalin-Vilkovisky Quantization, Comm. Math. Phys. 155 (1993) 249.

[219] A.Schwarz, Semiclassical Approximation in Batalin-Vilkovisky Formalism, Comm. Math. Phys. 158 (1993) 373.

[220] J.H. Schwarz, Superstring Theory, Phys. Rep. C89 (1982) 223.

[221] J.Schwinger, On the Green's Function of Quantized Fields. I., Proc. Nat. Acad. Sci. 37 (1951) 452;

On the Green's Function of Quantized Fields. II., Proc. Nat. Acad. Sci. 37 (1951) 455 .

[222] N. Seiberg and S. Shenker, A Note on Background (In-)dependence, Phys. Rev. D45 (1992) 4581.

[223] A. Sen, On the Background Independence of String Field Theory, Nucl. Phys. B345 (1990) 551;

On the Background Independence of String Field Theory II. Analysis of Onshell S-Matrix Elements, Nucl. Phys. B347 (1990) 270;

On the Background Independence of String Field Theory III. Explicit Field Redefinitions, Nucl. Phys. B391 (1993) 550.

[224] A. Sen and B. Zwiebach, A Proof of Local Background Independence of Classical Closed String Field Theory, Nucl. Phys. B414 (1994) 649.

[225] A. Sen and B.Zwiebach, Quantum Background Independence of Closed String Field Theory, Nucl. Phys. B414 (1994) 485.

[226] A. Sen and B.Zwiebach, A Note on Gauge Transformations in BatalinVilkovisky Theory, Phys. Lett. B320 (1994) 29. 
J. Gomis, J. París and S. Samuel - Antibracket, Antifields and ...

[227] E. Sezgin, Aspects of Kappa Symmetry, Texas A\&M preprint CTP-TAMU-2893, (Oct., 1993), hep-th/9310126.

[228] W. Siegel, Hidden Ghosts, Phys. Lett. 93B (1980) 170.

[229] W. Siegel, Covariantly Second-Quantized String, Phys. Lett. 142B (1984) 276; Covariantly Second-Quantized String II, Phys. Lett. 151B (1985) 391;

Covariantly Second-Quantized String III, Phys. Lett. 151B (1985) 396.

[230] W. Siegel, Batalin-Vilkovisky from Hamiltonian BRST, Int. J. Mod. Phys. A4 (1989) 3951.

[231] W. Siegel, Relation Between Batalin-Vilkovisky and First Quantized Style BRST, Int. J. Mod. Phys. A4 (1989) 3705.

[232] W. Siegel, Lorentz Covariant Gauges for Green-Schwarz Superstrings, in Strings 89, eds. R. Arnowitt et al. (World Scientific, Singapore, 1990).

[233] A. A. Slavnov, Ward Identities in Gauge Theories, Theor. Math. Phys. 10 (1972) 99.

[234] A. A. Slavnov, Physical Unitarity in the BRST Approach, Phys. Lett. B217 (1989) 91.

[235] A. A. Slavnov, BRST-Quantization and Unitarity, Nucl. Phys. B (Proc. Suppl.) B15 (1990) 107.

[236] M. F. Sohnius, Soft Gauge Algebras, Z. Phys. C18 (1983) 229.

[237] J. Stasheff, Closed String Field Theory, Strong Homotopy Lie Algebras and the Operad Actions of Moduli Space, Univ. of North Carolina preprint UNC-MATH93-1, (April, 1993), hep-th/9304061.

[238] G. Sterman, P. K. Townsend and P. van Nieuwenhuizen, Unitarity, Ward Identities, and New Quantization Rules of Supergravity, Phys. Rev. D17 (1978) 1501.

[239] Y. Takahashi, On the Generalized Ward Identity, Nuovo Cim. 6 (1957) 371.

[240] J. Tate, Homology of Noetherian Rings and Local Rings, Illinois J. Math. 1 (1957) 14.

[241] J.C. Taylor, Ward Identities and Charge Renormalization of the Yang-Mills Field, Nucl. Phys. B33 (1971) 436. 
[242] J. Thierry-Mieg, BRS Structure of the Antisymmetric Tensor Gauge Theories, Nucl. Phys. B335 (1990) 334.

[243] G.t'Hooft, Renormalization of Massless Yang-Mills Fields, Nucl. Phys. B33 (1971) 173.

[244] G.t'Hooft, Renormalizable Lagrangians for Massive Yang-Mills Fields, Nucl. Phys. B35 (1971) 167.

[245] G. t'Hooft and M. Veltman, Diagrammar, in Louvain 1973, Particle Interactions at Very High energies, Part B, (New York, 1973), p.177-322.

[246] C. B. Thorn, Perturbation Theory for Quantized String Fields, Nucl. Phys. B287 (1987) 61.

[247] C. B. Thorn, String Field Theory, Phys. Rep. 175 (1989) 1.

[248] M. Tonin, Covariant Quantization and Anomalies of the $G-S$ Heterotic $\sigma-$ Model, Int. J. Mod. Phys. A6 (1991) 315.

[249] M. Tonin, Dimensional Regularization and Anomalies in Chiral Gauge Theories, talk presented at Topical Workshop on Nonperturbative Aspect of Chiral Gauge Theories, Rome, Italy, (March, 1992), in Rome Chiral Gauge, p.137-151.

[250] P. K. Townsend, Gauge Invariance for Spin 1/2, Phys. Lett. 90B (1980) 275.

[251] W. Troost, P. van Nieuwenhuizen and A. Van Proeyen, Anomalies and the Batalin-Vilkovisky Lagrangian Formalism, Nucl. Phys. B333 (1990) 727.

[252] W. Troost and A. Van Proeyen, Regularization and the BV Formalism, in Proc. of Strings 93, Berkeley, (May, 1993).

[253] W. Troost and A. Van Proeyen, An Introduction to Batalin-Vilkovisky Lagrangian Quantisation, Leuven Notes in Math. Theor. Phys., in preparation.

[254] I. V. Tyutin, Gauge Invariance in Field Theory and Statistical Mechanics, Lebedev preprint $\mathrm{n}^{\circ} 39$ (1975), unpublished.

[255] I. V. Tyutin and Sh. Shvartsman, BRST Operator and Open Algebra, Phys. Lett. B169 (1986) 225.

[256] R. Utiyama, Invariant Theoretical Interpretation of Interaction, Phys. Rev. 101 (1956) 1597. 
[257] S. Vandoren and A. Van Proeyen, Simplifications in Lagrangian BV Quantization Exemplified by the Anomalies of Chiral $W_{3}$ Gravity, Nucl. Phys. B411 (1994) 257.

[258] P. van Nieuwenhuizen, Supergravity, Phys. Rep. 68 (1981) 189.

[259] A. Van Proeyen, Batalin-Vilkovisky Lagrangian Quantization, talk presented in Proc. of Strings and Symmetries Stony Brook, Strings: Stony Brook, 1991, p.388-406.

[260] M. A. Vasil'ev, "Gauge" Form of Description of Massless Fields With Arbitrary Spin, Sov. J. Nucl. Phys. 32 (1980) 439.

[261] E. Verlinde, The Master Equation of String Theory, Nucl. Phys. B381 (1992) 141.

[262] B. L. Voronov, P. M. Lavrov and I.V.Tyutin, Canonical Transformations and Gauge Dependence in General Gauge Theories, Sov. J. Nucl. Phys. 36 (1982) 292.

[263] B.L.Voronov and I.V. Tyutin, Formulation of Gauge Theories of General Form. I, Theor. Math. Phys. 50 (1982) 218.

[264] B. L. Voronov and I. V. Tyutin, Formulation of Gauge Theories of General Form. II. Gauge-Invariant Renormalizability and Renormalization Structure, Theor. Math. Phys. 50 (1982) 628.

[265] J. C. Ward, An Identity in Quantum Electrodynamics, Phys. Rev. 78 (1950) 182.

[266] S. Weinberg, A Model of Leptons, Phys. Rev. Lett. 19 (1967) 1264.

[267] J. Wess and J. Bagger, Introduction to Supersymmetry, (Princeton Univ. Press, Princeton, NJ, 1983).

[268] J.Wess and B.Zumino, Consequences of Anomalous Ward Identities, Phys. Lett. 37B (1971) 95.

[269] P. L. White, Anomaly Consistency Conditions to All Orders, Phys. Lett. B284 (1992) 55.

[270] E. Witten, Non-Commutative Geometry and String Field Theory, Nucl. Phys. B268 (1986) 253.

[271] E. Witten, Open Superstrings, Nucl. Phys. B276 (1986) 291. 
J. Gomis, J. París and S. Samuel - Antibracket, Antifields and ...

[272] E. Witten, Topological Quantum Field Theory, Comm. Math. Phys. 117 (1988) 353.

[273] E. Witten, A Note on the Antibracket Formalism, Mod. Phys. Lett. A5 (1990) 487.

[274] E. Witten, On Background Independent Open String Field Theory, Phys. Rev. D46 (1992) 5467.

[275] E. Witten, Some Computations in Background Independent Off-Shell String Field Theory, Phys. Rev. D47 (1993) 5467.

[276] C. N. Yang and R. L. Mills, Considerations of Isotopic Spin and Isotopic Gauge Invariance, Phys. Rev. 96 (1954) 191.

[277] J.Zinn-Justin, Renormalization of Gauge Theories, in Trends in Elementary Particle Theory, edited by H. Rollnik and K. Dietz, Lecture Notes in Physics, Vol 37, (Springer-Verlag, Berlin, 1975).

[278] J.Zinn-Justin, Quantum Field Theory and Critical Phenomena, (Oxford University Press, Oxford, England, 1989).

[279] B.Zumino, Chiral Anomalies and Differential Geometry, in Relativity, Groups and Topology, edited by B.S. deWitt and R.Stora, (North-Holland, Amsterdam, 1983), and references therein.

[280] B.Zwiebach, Quantum Closed Strings from Minimal Area, Int. J. Mod. Phys. A5 (1990) 275;

Consistency of Closed String Polyhedra from Minimal Area, Phys. Lett. B241 (1990) 343;

How Covariant Closed String Theory Solves a Minimal Area Problem, Comm. Math. Phys. 136 (1991) 83.

[281] B. Zwiebach, Closed String Field Theory: An Introduction, MIT preprint MITCTP-2206, (May, 1993), hep-th/9305026.

[282] B. Zwiebach, Closed String Field Theory: Quantum Action and the B-V Master Equation, Nucl. Phys. B390 (1993) 33. 